



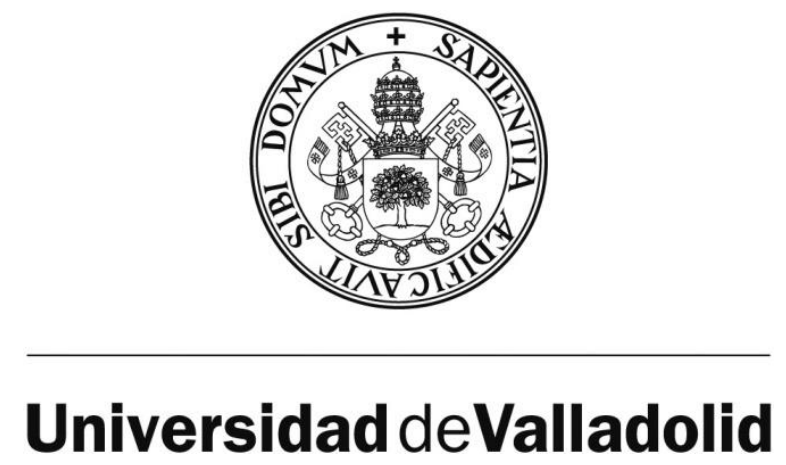

FACULTAD DE CIENCIAS

DEPARTAMENTO

Física de la Materia Condensada

TESIS DOCTORAL

\section{DEVELOPMENT OF A MARS SIMULATION CHAMBER IN SUPPORT FOR THE SCIENCE ASSOCIATED TO THE RAMAN LASER SPECTROMETER (RLS) INSTRUMENT FOR ESA'S EXOMARS MISSION}

Presentada por ALEJANDRO CATALÁ ESPÍ para optar al grado de doctor por la Universidad de Valladolid Dirigida por:

Fernando Rull Pérez 





\section{ACKNOWLEDGMENTS}

Mi más sincero agradecimiento a todas aquellas personas que han hecho posible que esta tesis doctoral se desarrollara. En particular:

A mi director de tesis el Catedrático Fernando Rull Pérez, por darme la oportunidad de realizar esta tesis en sus instalaciones y estancias en centros de renombre en el extranjero, todo ello mediante la concesión de una beca de formación FPI.

A mis compañeros de la Unidad Asociada UVa-CSIC-CAB, con especial mención a Alberto Vegas y Aurelio Sanz, por su sabiduría infinita, ayuda y disponibilidad, y a Gloria Venegas por su apoyo y complementación en aquellos aspectos ajenos a mi formación y con quien ahora tengo el placer de trabajar.

A la gente de INTA, de quienes he aprendido un poco de rocket science pero sobre todo ¡a generar documentación!

A todos aquellos colaboradores que han hecho que las estancias en el extranjero me ayudasen a complementar mi formación como investigador en el campo de la exploración planetaria, en especial a Pablo Sobrón y a Ian Hutchinson.

Y por último, pero no por ello menos importante, a mi familia por el apoyo, cariño y ánimo recibidos desde la distancia. Sin ellos nada de esto hubiese sido posible. 



\section{TABLE OF CONTENTS}

ACKNOWLEDGMENTS

TABLE OF CONTENTS

III

TABLA DE CONTENIDOS

VII

INDEX OF FIGURES

$\mathbf{X I}$

INDEX OF TABLES

XV

CHAPTER 1 - INTRODUCTION

1.1. Planetary Exploration of Mars

1.2. Astrobiology and Life

1.3. The ExoMars Programme and the 2018 ExoMars Rover

1.4. RLS-ExoMars and Raman spectroscopy

CHAPTER 2 - JUSTIFICATION AND OBJECTIVES 
3.2.1.3. Pressure sensor

3.2.1.4. Gas and valves

3.2.1.5. Pressure controller

3.2.1.6. Refrigeration unit

3.2.2. Pressure and temperature test

3.3.1.1. Refillable Container (RC)

3.3.1.2. Flattening subsystem

3.3.1.3. Optical Window

4.3.1. XYZ positioning system 59

$\begin{array}{ll}\text { 4.3.2. Optical system } & 60\end{array}$

4.3.2.1. Cube-Mounted pellicle beamsplitter $\quad 61$

4.3.2.2. Raman Optical Head (OH) cylindrical adapter $\quad 62$

4.3.2.3. Microscope objective ring adapter 63

$\begin{array}{ll}\text { 4.3.2.4. Visual aid system } & 64\end{array}$

$\begin{array}{lc}\text { 4.4. Tests } & 65\end{array}$

4.4.1. Laser power output $\quad 65$

4.4.2. Laser spot size $\quad 67$

$\begin{array}{ll}\text { 4.4.3. Laser irradiance } & 70\end{array}$

4.4.4. Depth of Field (DOF) 71

$\begin{array}{ll}\text { 4.4.5. Spectral resolution } & 73\end{array}$

4.4.6. Wavelength calibration $\quad 74$

4.4.7. Optical system transfer function and intensity calibration $\quad 75$

$\begin{array}{ll}\text { CHAPTER } 5 \text { - EXPERIMENTS } & 77\end{array}$

$\begin{array}{lr}\text { 5.1. Introduction } & 79\end{array}$ 
5.3. Experiment 1: Sweep in environmental conditions

5.3.1. Methodology

5.3.2. Samples

5.3.2.1. Alunite

5.3.2.2. Gypsum

5.3.2.3. Jarosite

5.4. Experiment 2: Sweep in power

5.4.1. Methodology

5.4.2. Samples

5.4.2.1. Jarosite

5.4.2.2. Hematite

5.5. Experiment 3: Sweep in spot size

5.5.1. Methodology

5.5.2. Samples

5.5.2.1. Jarosite 
Oral Communications

Merits, Honours and transversal activities

References 


\section{TABLA DE CONTENIDOS}

AGRADECIMIENTOS

TABLE OF CONTENTS

III

TABLA DE CONTENIDOS

VII

ÍNDICE DE FIGURAS

$\mathbf{X I}$

ÍNDICE DE TABLAS

XV

CAPÍTULO 1 - INTRODUCCION

1.1. Exploración Planetaria de Marte

1.2. Astrobiología y Vida

1.3. El Programa ExoMars y el Rover de ExoMars 2018

1.4. RLS-ExoMars y la espectroscopia Raman

CAPÍTULO 2 - JUSTIFICACIÓN Y OBJETIVOS 
3.2.1.2. Estación de generación de vacío

3.2.1.3. Sensor de presión 32

3.2.1.4. Gas y válvulas 33

3.2.1.5. Controlador de presión 34

3.2.1.6. Unidad de refrigeración 35

$\begin{array}{ll}\text { 3.2.2. Test de presión y temperatura } & 37\end{array}$

$\begin{array}{ll}\text { 3.3. Sistema de muestra } & 40\end{array}$

$\begin{array}{ll}\text { 3.3.1. Partes } & 41\end{array}$

3.3.1.1. Contenedor Rellenable (RC) 41

3.3.1.2. Subsistema de aplanado $\quad 44$

3.3.1.3. Ventana Óptica $\quad 46$

$\begin{array}{ll}\text { 3.3.2. Test del sistema de muestra } & 48\end{array}$

CAPÍTULO 4 - SIMULADOR DE CIENCIA DE RLS-EXOMARS 53

4.1. Introducción $\quad 55$

4.2. Antecedentes: ExoMars RLS Simulator $\quad 57$

4.3. Simulador de Ciencia de RLS-ExoMars $\quad 59$

4.3.1. Sistema de posicionamiento XYZ 59

4.3.2. Sistema óptico $\quad 60$

4.3.2.1. Divisor de haz de película montado en cubo 61

4.3.2.2. Adaptador cilíndrico de Cabezal Óptico Raman (OH) 62

4.3.2.3. Anillo adaptador de objetivo de microscopio 63

$\begin{array}{ll}\text { 4.3.2.4. Sistema de ayuda visual } & 64\end{array}$

4.4. Tests

4.4.1. Potencia de salida del láser $\quad 65$

4.4.2. Tamaño de spot láser $\quad 67$

4.4.3. Irradiancia láser $\quad 70$

4.4.4. Profundidad de campo (DOF) 71

4.4.5. Resolución espectral

4.4.6. Calibración en longitud de onda $\quad 74$

4.4.7. Función de transferencia del sistema óptico y calibración en intensidad 75

CAPÍTULO 5 - EXPERIMENTOS

$\begin{array}{ll}\text { 5.1. Introducción } & 79\end{array}$ 
5.3. Experimento 1: Barrido en condiciones ambientales

5.3.1. Metodología

5.3.2. Muestras

5.3.2.1. Alunita

5.3.2.2. Yeso

5.3.2.3. Jarosita

5.3.2.4. Cuarzo

5.4. Experimento 2: Barrido en potencia

5.4.1. Metodología

5.4.2. Muestras

5.4.2.1. Jarosita

5.4.2.2. Hematite

5.5. Experimento 3: Barrido en tamaño de spot

5.5.1. Metodología

5.5.2. Muestras

5.5.2.1. Jarosita 
Abstracts

Comunicaciones orales

Méritos, Honores y actividades transversales

Referencias 


\section{INDEX OF FIGURES}

Figure 1.1. - Map of the planet Mars (1877-1888) by Giovanni Schiaparelli (Credit: International

Planetary Cartography Database)

Figure 1.2. - Misinterpretation of Schiaparelli's 'channels' by Percival Lowell (Credit: The New York

Times, December 9, 1906)

Figure 1.3. - Three science fiction books related to Mars's intelligent beings. (a) The War of The

World by H.G.Wells (public domain image), (b) A Princess of Mars, the first book of the

Barsoom series by Edgar Rice Burroughs (public domain image), and (c) The Martian

Chronicles by Ray Bradbury (Credit: Doubleday publishing company)

Figure 1.4. - Images of the Mars surface by (a) Mariner 4 (Credit: NASA) and (b) Mariner 6 and 7

(Credit: NASA)

Figure 1.5. - Viking 2 panorama taken at its landing site (Credit: Edward A. Guinness, Washington

University in St. Louis)

Figure 1.6. - Sojourner rover analyzing Yogi rock (Credit: IMP Team, JPL, NASA)

Figure 1.7. - Comanche outcrop (at the back) found by the Spirit rover on Mars. It is composed, in part, of Fe-Mg carbonates. (Credit: NASA/JPL-Caltech/Cornell University)

Figure 1.8. - Gale crater and the landing elipse for MSL. Gale crater is a layered mound of clays and sulphates. (Credit: NASA / JPL-Caltech / ESA / DLR / FU Berlin / MSSS)

Figure 1.9. - Mars global map. A clear difference in height can be observed between smooth northern highlands and the rugged southern lowlands. (Credit: MOLA Science Team)

Figure 1.10. - SEM image for ALH84001 interpreted biogenic microstructures (Credit: NASA)

Figure 1.11. - Elements of the ExoMars Programme: the Trace Gas Orbiter (TGO) on the upper left and the ExoMars Rover on the lower right. (Credit: ESA)

Figure 1.12. - Artist conception of the ExoMars rover drilling down to -2m in the subsurface of Mars. (Credit: ESA)

Figure 1.13. Examples of (a) core samples obtained with the (b) ExoMars drill unit. (Credit: ESA) 15

Figure 1.14. - The PSDDS loaded with sample. The RC can be seen just below it, on the PSHS. (Credit: Kayser-Threde GmbH.)

Figure 1.15. - RLS-ExoMars instrument layout with its three components: ICEU, SPU and iOH.

(Credit: ESA) 
Figure 1.16. - Diagram of the energy levels and transitions for IR, Rayleigh elastic scattering and Raman Stokes and Anti-Stokes inelastic scattering. (Credit: Moxfyre / Wikipedia)

Figure 1.17. - Raman spectrum of Cyclohexane. (Credit: McCreery Research Group, National Institute for Nanotechnology, University of Alberta)

Figure 3.1. - (a) 3D layout and (b) real image of the RLS-ExoMars Simulation Chamber + RLS-

ExoMars Science Simulator

Figure 3.2. - Scheme of the elements of the RLS-ExoMars Simulation Chamber 28

Figure 3.3. - 3D layout of the chamber body, a 6" spherical octagon. (Credit: Kimball Physics) 30

Figure 3.4. - HiCube Eco 80 from (a) catalogue (Credit: Pfeiffer Vacuum) and (b) RLS-ExoMars Simulation Chamber assembly

Figure 3.5. - Vacuum hose attached to one of the DN40CF lateral ports of the chamber body

Figure 3.6. - TPR 280 Pirani sensor for the pressure measurement inside the RLS-ExoMars

Simulation Chamber.

Figure 3.7. - Pressure curves for the TPR280 pressure sensor depending on the gas being used.

(Credit: Pfeiffer Vacuum)

Figure 3.8. - (a) 30 $\mathrm{L} \mathrm{CO}_{2}$ gas bottle and (b) detail of the pressure regulation valves and the rubber tube

Figure 3.9. - (a) Butterfly valve acting as an up-to-air valve and (b) butterfly valve in line with the gas flow valve and the gas line coming from the $\mathrm{CO}_{2}$ gas bottle

Figure 3.10. - RVC 300 pressure controller by Pfeiffer Vacuum 35

Figure 3.11. - Huber Ministat 230 with Pilot ONE controller.

Figure 3.12. - Fluid feedthrough by MDC Vacuum with two lines isolated with neoprene. The red tubes on the lower right part of the image are the $1.5 \mathrm{~m}$-long isolated stainless steel hoses. 36

Figure 3.13. - LEMO feedthrough for the temperature measurement of the process on the RC through a PT100 sensor.

Figure 3.14. - Pressure and temperature curves without thermal load on the RC 38

Figure 3.15. - RC built by KT (Credit: Kayser-Threde GmbH.) 41

Figure 3.16. - RLS-ExoMars Simulation Chamber: detail of the interior. 42

Figure 3.17. - Exploded view of the RC inside the RLS-ExoMars Simulation Chamber 43

Figure 3.18. - Front view of the RC inside the RLS-ExoMars Simulation Chamber. 43

Figure 3.19. - 3D layout of the linear manipulator and the blade attached to it. 44

Figure 3.20. - Mechanically actuated linear manipulator by MDC vacuum, attached to the $R L S$ -

ExoMars Simulation Chamber.

Figure 3.21. - Stepper motor controller for the linear manipulator of the flattening subsystem. 46

Figure 3.22. - Exploded view of the top cover of the RLS-ExoMars Simulation Chamber hosting a thin optical window.

Figure 3.23. - Transmission curve of the optical window used for the RLS-ExoMars Simulation

Chamber.

Figure 3.24. - Detail view of the optical window assembly integrated with the top cover of the $R L S$ - 
Figure 3.25. - Image of quartz sample before (a) and after (b) the flattening process

Figure 3.26. - Focused positions for the quartz mineral grains of three lines along $10 \mathrm{~mm}$ of the RC.

Line 1 is the central line $+500 \mu \mathrm{m}$; Line 2 is the central line; and Line 3 is the central line $500 \mu \mathrm{m}$.

Figure 3.27. - Image of gypsum sample before (a) and after (b) the flattening process

Figure 3.28. - Focused positions for the gypsum mineral grains of three lines along $10 \mathrm{~mm}$ of the RC.

Line 1 is the central line $+500 \mu \mathrm{m}$; Line 2 is the central line; and Line 3 is the central line $500 \mu \mathrm{m}$.

Figure 4.1. - Drawing of the IOH of RLS and its optical components. (Credit: ESA) 56

Figure 4.2. ExoMars RLS Simulator and parts (from López-Reyes et al., 2013). 57

Figure 4.3. - RLS-ExoMars Simulation Chamber + RLS-ExoMars Science Simulator 59

Figure 4.4. - (a) PT3-Z8 XYZ motorized stage and (b) TDC001 DC servo controller. (Credit: Thorlabs, Inc.)

Figure 4.5. - 3D layout of the optical system of the new RLS-ExoMars Science Simulator (Credit for individual 3D parts: Thorlabs, Inc.)

Figure 4.6. - Scheme of the pellicle beamsplitter used to divide light (Credit: Thorlabs, Inc.) 62

Figure 4.7. - SM1P1 by Thorlabs used as Raman OH cylindrical adapter (Credit: Thorlabs, Inc.) 62

Figure 4.8. - SM1A12 ring adapter for M25x0.75 microscope objectives (Credit: Thorlabs, Inc.) 63

Figure 4.9. - Visual aid system optical assembly (Credit of the images for the individual components:

Thorlabs, Inc.)

Figure 4.10. - Average output power as a function of the percentage of the maximum input power,

from $0 \%$ to $100 \%$ in $5 \%$ steps. $\quad 67$

Figure 4.11. - Graph presenting the data collected in Table 4.2 69

Figure 4.12. - Screenshot of the software for measuring the laser beam profile during the measurement of the spot in focus $\quad 69$

Figure 4.13. - DOF for the 50x microscope objective $\quad 72$

Figure 4.14. - Normalized transfer function of the system $\quad 76$

Figure 5.1.a - Low Raman shift region $\left(100-1300 \mathrm{~cm}^{-1}\right)$ of Alunite spectra taken at different environmental conditions $\quad 84$

Figure 5.1.b - High Raman shift region (3000-4000 $\left.\mathrm{cm}^{-1}\right)$ of Alunite spectra taken at different environmental conditions

Figure 5.2.a. - Low Raman shift region (100-1300 $\left.\mathrm{cm}^{-1}\right)$ of Gypsum spectra taken at different environmental conditions

Figure 5.2.b. - High Raman shift region (3000-4000 $\left.\mathrm{cm}^{-1}\right)$ of Gypsum spectra taken at different environmental conditions

Figure 5.3.a. - Low Raman shift range (100-1300 $\left.\mathrm{cm}^{-1}\right)$ of Jarosite spectra taken at different environmental conditions

Figure 5.3.b. - High Raman shift range (3000-4000 $\left.\mathrm{cm}^{-1}\right)$ of Jarosite spectra taken at different environmental conditions 
Figure 5.4. - Low Raman shift region (100-1300 $\left.\mathrm{cm}^{-1}\right)$ of Quartz spectra taken at different environmental conditions

Figure 5.5. - Pressure and temperature curves for the period of achievement of the RLS-ExoMars relevant conditions. The pressure axis has been cropped to show the 'important' region.

Figure 5.6. - Jarosite before (left) and after (right) flattening under RLS-ExoMars relevant conditions.

Figure 5.7. - Vertical profile of three lines in the central region of the RC

Figure 5.8. - Pressure and temperature curves for the period of achievement of the RLS-ExoMars relevant conditions. The pressure axis has been cropped to show the 'important' region. 120 Figure 5.9. - Hematite before (left) and after (right) the flattening process inside the RLS-ExoMars

Chamber.

Figure 5.10. - Vertical profile of three lines in the central region of the RC.

Figure 5.11. - Spectra of Gossan (Hematite + Goethite) for $1.2 \mathrm{~kW} / \mathrm{cm}^{2}$ (top) and $0.2 \mathrm{~kW} / \mathrm{cm}^{2}$

(bottom). The latter was acquired later. The Raman peaks are recovered, as the grain has not been irreversibly damaged with the high irradiance. (Credit: LINES, INTA)

Figure A.1. - Screen capture of the PC Software for controlling the RLS-ExoMars Simulation Chamber and the RLS-ExoMars Science Simulator

Figure B.1. - Stepper motor pinout (a) and table of states (b). (Credit: MDC Vacuum Products, LLC)

Figure B.2. - Circuit board with functional groups of components 


\section{INDEX OF TABLES}

Table 3.1. - Statistics for the three lines of points on the quartz sample after the flattening process.

Table 3.2. - Statistics for the three lines of points on the gypsum sample after the flattening process.

Table 4.1. - Average output power values as a function of the input power 66

Table 4.2. - Laser spot diameter (measured and fitted) as a function of the height from focus 68 Table 4.3. - Irradiance values calculated from the spot sizes (D) at different heights (H) and laser powers measured in the previous sections. The two cells coloured in green and red mark the current lower and upper limits of irradiance defined for the RLS instrument.

Table 4.4. - Spectral resolutions in Raman shift for the converted peaks of the calibration lamps $\begin{array}{ll}\text { along the covered spectral range. } & 74\end{array}$

Table 5.1. - Positions and widths of the main Raman peaks of Alunite 86

Table 5.2. - Positions and widths of the main Raman peaks of Gypsum 89

Table 5.3. - Positions and widths of the main Raman peaks of Jarosite 92

Table 5.4. - Positions and widths of the main Raman peaks of Quartz 94

Table 5.5. - Percentage of points within $100 \mu \mathrm{m}$ and $200 \mu \mathrm{m}$ as well as mean height and standard deviation for the grains in the three profiled lines 99

Table 5.6. - Irradiance values, images after exposure to laser and corresponding Raman spectra for the selected point \#1. Blue:0.051kW/ $\mathrm{cm}^{2}$; Red:0.127kW $/ \mathrm{cm}^{2}$; Green:0.203kW/ $/ \mathrm{cm}^{2}$; Yellow:0.287kW/cm²; Magenta: 0.363kW/ $\mathrm{cm}^{2}$; Black:0.394kW $/ \mathrm{cm}^{2}$.

Table 5.7. - Irradiance values, images after exposure to laser and corresponding Raman spectra for the selected point \#2. Blue:0.014kW/ $\mathrm{cm}^{2}$; Red:0.051kW $/ \mathrm{cm}^{2}$; Green:0.127kW/ $\mathrm{cm}^{2}$; Yellow:0.203kW/ $\mathrm{cm}^{2}$.

Table 5.8. - Irradiance values, images after exposure to laser and corresponding Raman spectra for the selected point \#2. Blue:0.014kW/ $\mathrm{cm}^{2}$; Red:0.051kW $/ \mathrm{cm}^{2}$; Green:0.127kW/ $/ \mathrm{cm}^{2}$; Yellow:0.203kW/ $\mathrm{cm}^{2}$; Magenta:0.287kW/ $\mathrm{cm}^{2}$.

Table 5.9. - Irradiance values, images after exposure to laser and corresponding Raman spectra for the selected point \#4. Blue:0.014kW/ $\mathrm{cm}^{2}$; Red:0.051kW $/ \mathrm{cm}^{2}$; Green:0.127kW/ $/ \mathrm{cm}^{2}$; Yellow:0.203kW/ $\mathrm{cm}^{2}$. 
Table 5.10. - Irradiance values, images after exposure to laser and corresponding Raman spectra for the selected point \#1. Blue:0.051kW/ $\mathrm{cm}^{2}$; Red:0.127kW $/ \mathrm{cm}^{2}$; Green:0.203kW/ $/ \mathrm{cm}^{2}$; Yellow:0.287kW/cm²; Magenta: 0.363kW/cm²; Black:0.452kW/cm²; Cyan:0.529kW/cm². 104

Table 5.11. - SNR values for the v1 peak of jarosite in all the RLS-ExoMars-like previous spectra, and their corresponding irradiance values and associated powers. Cells highlighted in green show best SNR values for each point.

106

Table 5.12. - Irradiance values, images after exposure to laser and corresponding Raman spectra for the selected point \#6. Blue:0.014kW/ $\mathrm{cm}^{2}$; Red:0.051kW $/ \mathrm{cm}^{2}$; Green:0.127kW/ $/ \mathrm{cm}^{2}$; Yellow:0.203kW/cm²; Magenta:0.287kW/ $/ \mathrm{cm}^{2}$; Black: $0.363 \mathrm{~kW} / \mathrm{cm}^{2}$.

Table 5.13. - Irradiance values, images after exposure to laser and corresponding Raman spectra for the selected point \#6. Blue:0.452kW/ $\mathrm{cm}^{2}$; Red:0.529kW $/ \mathrm{cm}^{2}$; Green:0.605kW/ $/ \mathrm{cm}^{2}$; Yellow:0.689kW/cm²; Magenta:0.763kW/cm²; Black: 0.841kW/ $\mathrm{cm}^{2}$.

Table 5.14. - Irradiance values, images after exposure to laser and corresponding Raman spectra for the selected point \#7. Blue:0.014kW/ $\mathrm{cm}^{2}$; Red:0.051kW $/ \mathrm{cm}^{2} ;$ Green:0.127kW $/ \mathrm{cm}^{2}$; Yellow:0.203kW/cm²; Magenta:0.287kW/ $\mathrm{cm}^{2}$; Black: $0.363 \mathrm{~kW} / \mathrm{cm}^{2}$.

Table 5.15. - Irradiance values, images after exposure to laser and corresponding Raman spectra for the selected point \#7. Blue:0.452kW/ $\mathrm{cm}^{2}$; Red:0.529kW $/ \mathrm{cm}^{2}$; Green:0.605kW/ $/ \mathrm{cm}^{2}$; Yellow:0.689kW/ $\mathrm{cm}^{2}$; Magenta:0.763kW/ $\mathrm{cm}^{2}$; Black: $0.841 \mathrm{~kW} / \mathrm{cm}^{2}$.

Table 5.16. - Irradiance values, images after exposure to laser and corresponding Raman spectra for the selected point \#8. Blue:0.014kW/ $\mathrm{cm}^{2}$; Red:0.051kW $/ \mathrm{cm}^{2}$; Green:0.127kW/ $/ \mathrm{cm}^{2}$; Yellow:0.203kW/ $\mathrm{cm}^{2}$; Magenta:0.287kW/ $\mathrm{cm}^{2}$; Black: $0.363 \mathrm{~kW} / \mathrm{cm}^{2}$.

Table 5.17. - Irradiance values, images after exposure to laser and corresponding Raman spectra for the selected point \#8. Blue:0.452kW/ $\mathrm{cm}^{2}$; Red:0.529kW $/ \mathrm{cm}^{2}$; Green:0.605kW/ $/ \mathrm{cm}^{2}$; Yellow:0.689kW/cm²; Magenta:0.763kW/ $\mathrm{cm}^{2}$.

Table 5.18. - Irradiance values, images after exposure to laser and corresponding Raman spectra for the selected point \#9. Blue:0.051kW/ $\mathrm{cm}^{2}$; Red:0.127kW $/ \mathrm{cm}^{2}$; Green:0.203kW/ $/ \mathrm{cm}^{2}$; Yellow:0.287kW/cm²; Magenta: 0.363kW/cm²; Black:0.452kW/cm²; Cyan:0.529kW/cm². 114

Table 5.19. - Irradiance values, images after exposure to laser and corresponding Raman spectra for the selected point \#9. Blue:0.605kW/ $\mathrm{cm}^{2}$; Red:0.689kW $/ \mathrm{cm}^{2}$; Green:0.763kW/ $/ \mathrm{cm}^{2}$; Yellow:0.841kW/cm²; Magenta: 0.926kW/cm²; Black:1.01kW/cm².

Table 5.20. - Irradiance values, images after exposure to laser and corresponding Raman spectra for the selected point \#10. Blue:0.051kW/ $\mathrm{cm}^{2}$; Red:0.127kW/ $\mathrm{cm}^{2} ;$ Green:0.203kW/ $/ \mathrm{cm}^{2}$; Yellow:0.287kW/cm²; Magenta: $0.363 \mathrm{~kW} / \mathrm{cm}^{2}$.

Table 5.21. - Irradiance values, images after exposure to laser and corresponding Raman spectra for the selected point \#10. Blue:0.452kW/ $\mathrm{cm}^{2}$; Red:0.529kW/ $\mathrm{cm}^{2} ;$ Green:0.605kW/ $\mathrm{cm}^{2}$; Yellow: $0.689 \mathrm{~kW} / \mathrm{cm}^{2}$.

Table 5.22. - SNR values for the v1 peak of jarosite in all the Earth-like previous spectra with the corresponding irradiances and associated powers. The cells marked in green correspond to maximum SNR values. Those marked in yellow correspond to a mineral that is not jarosite. It may be another mineral below the first jarosite layers or a transformation from jarosite. 119 
Table 5.23. - Percentage of points within $100 \mu \mathrm{m}$ and $200 \mu \mathrm{m}$ as well as mean height and standard deviation for the grains in the three profiled lines

Table 5.24. - Irradiance values, images after exposure to laser and corresponding Raman spectra for the selected point \#1. Blue:0.014kW/ $\mathrm{cm}^{2}$; Red:0.051kW/ $\mathrm{cm}^{2}$; Green:0.127kW/ $/ \mathrm{cm}^{2}$.

Table 5.25. - Irradiance values, images after exposure to laser and corresponding Raman spectra for the selected point \#2. Blue:0.014kW/cm²; Red:0.051kW/cm²; Green:0.127kW/cm².

Table 5.26. - Irradiance values, images after exposure to laser and corresponding Raman spectra for the selected point \#3. Blue:0.014kW/ $\mathrm{cm}^{2}$; Red:0.051kW $/ \mathrm{cm}^{2}$; Green:0.127kW $/ \mathrm{cm}^{2}$.

Table 5.27. - Irradiance values, images after exposure to laser and corresponding Raman spectra for the selected point \#4. Blue:0.014kW/ $\mathrm{cm}^{2}$; Red:0.051kW/ $\mathrm{cm}^{2}$; Green:0.127kW/ $/ \mathrm{cm}^{2}$.

Table 5.28. - Irradiance values, images after exposure to laser and corresponding Raman spectra for the selected point \#5. Blue:0.014kW/ $\mathrm{cm}^{2}$; Red:0.051kW/ $/ \mathrm{cm}^{2}$; Green:0.127kW/ $/ \mathrm{cm}^{2}$.

Table 5.29. - SNR values, irradiances and associated powers for the $405 \mathrm{~cm}^{-1}$ peak of hematite in all the RLS-ExoMars-like previous spectra. Cells marked in green show the best SNR values. 128

Table 5.30. - Irradiance values, images after exposure to laser and corresponding Raman spectra for the selected point \#6. Blue:0.051kW/ $\mathrm{cm}^{2}$; Red:0.127kW $/ \mathrm{cm}^{2}$; Green:0.203kW/ $/ \mathrm{cm}^{2}$; Yellow:0.287kW/cm²; Magenta:0.363kW/ $\mathrm{cm}^{2}$; Black:0.452kW/ $\mathrm{cm}^{2}$.

Table 5.31. - Irradiance values, images after exposure to laser and corresponding Raman spectra for the selected point \#7. Blue:0.051kW/ $\mathrm{cm}^{2}$; Red:0.127kW $/ \mathrm{cm}^{2}$; Green:0.203kW/ $/ \mathrm{cm}^{2}$; Yellow:0.287kW/cm²; Magenta:0.363kW/ $\mathrm{cm}^{2}$; Black:0.452kW/ $\mathrm{cm}^{2}$.

Table 5.32. - Irradiance values, images after exposure to laser and corresponding Raman spectra for the selected point \#8. Blue:0.051kW/ $\mathrm{cm}^{2}$; Red:0.127kW $/ \mathrm{cm}^{2}$; Green:0.203kW/ $\mathrm{cm}^{2}$; Yellow:0.287kW/cm²; Magenta:0.363kW/cm²; Black:0.452kW/cm².

Table 5.33. - Irradiance values, images after exposure to laser and corresponding Raman spectra for the selected point \#9. Blue:0.051kW/ $\mathrm{cm}^{2}$; Red:0.127kW/ $\mathrm{cm}^{2} ;$ Green:0.203kW/ $/ \mathrm{cm}^{2}$; Yellow:0.287kW/cm²; Magenta:0.363kW/ $\mathrm{cm}^{2}$.

Table 5.34. - Irradiance values, images after exposure to laser and corresponding Raman spectra for the selected point \#10. Blue:0.051kW/ $\mathrm{cm}^{2}$; Red:0.127kW/ $\mathrm{cm}^{2} ;$ Green:0.203kW/ $/ \mathrm{cm}^{2}$; Yellow:0.287kW/cm²; Magenta:0.363kW/ $\mathrm{cm}^{2}$.

Table 5.35. - SNR values for the $405 \mathrm{~cm}^{-1}$ peak of hematite in all the Earth-like previous spectra. 134 Table 5.36. - Height values from focus, images after exposure to laser and corresponding Raman

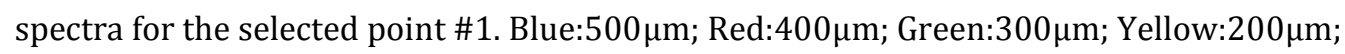
Magenta: $100 \mu \mathrm{m}$; Black: $50 \mu \mathrm{m}$.

Table 5.37. - Height values from focus, images after exposure to laser and corresponding Raman

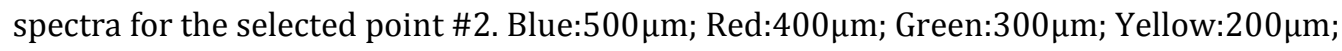
Magenta: $100 \mu \mathrm{m}$.

Table 5.38. - Height values from focus, images after exposure to laser and corresponding Raman

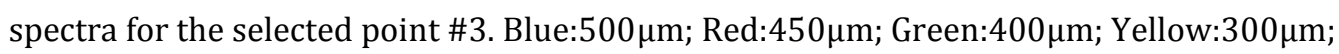
Magenta: $200 \mu \mathrm{m}$. 
Table 5.39. - Height values from focus, images after exposure to laser and corresponding Raman

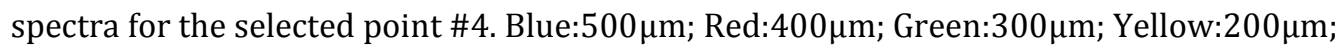
Magenta: $100 \mu \mathrm{m}$.

Table 5.40. - Height values from focus, images after exposure to laser and corresponding Raman

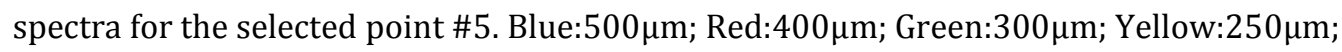
Magenta: $200 \mu \mathrm{m}$.

Table 5.41. - SNR values for the v1 peak of jarosite in all the RLS-ExoMars-like previous spectra. Height from focus, spot size at that height and irradiance is also presented in it. Green cells mark the best SNR values for each point.

Table 5.42. - Height values from focus, images after exposure to laser and corresponding Raman

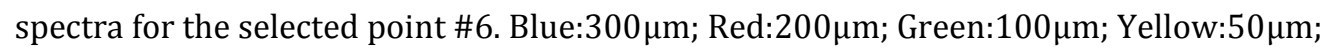
Magenta: $0 \mu \mathrm{m}$.

Table 5.43. - Height values from focus, images after exposure to laser and corresponding Raman

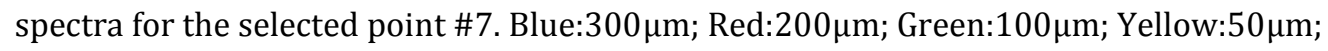
Magenta: $0 \mu \mathrm{m}$.

Table 5.44. - Height values from focus, images after exposure to laser and corresponding Raman

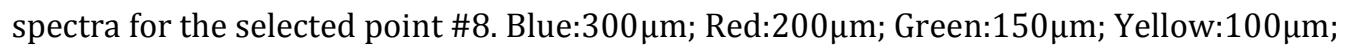
Magenta: $50 \mu \mathrm{m}$.

Table 5.45. - Height values from focus, images after exposure to laser and corresponding Raman

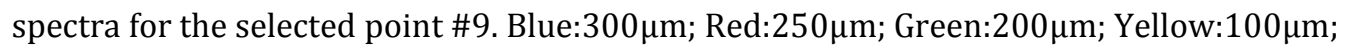
Magenta:50 $\mu \mathrm{m}$.

Table 5.46. - Height values from focus, images after exposure to laser and corresponding Raman

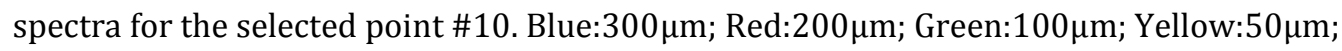
Magenta:0 $\mu \mathrm{m}$.

Table 5.47. - SNR values for the v1 peak of jarosite in all the Earth-like previous spectra. Height from focus, spot size at that height and irradiance is also presented in it. Cells marked in green correspond to the best SNR values for each point.

Table 5.48. - Height values from focus, images after exposure to laser and corresponding Raman spectra for the selected point \#1. Blue:500 $\mu \mathrm{m}$; Red: $400 \mu \mathrm{m}$; Green:300 $\mu \mathrm{m}$.

Table 5.49. - Height values from focus, images after exposure to laser and corresponding Raman spectra for the selected point \#2. Blue: $400 \mu \mathrm{m}$; Red:300 $\mu \mathrm{m}$; Green:250 $\mu \mathrm{m}$.

Table 5.50. - Height values from focus, images after exposure to laser and corresponding Raman spectra for the selected point \#3. Blue:500 $\mu \mathrm{m}$; Red: $400 \mu \mathrm{m}$; Green:300 $\mu \mathrm{m}$.

Table 5.51. - Height values from focus, images after exposure to laser and corresponding Raman spectra for the selected point \#4. Blue:500 $\mu \mathrm{m}$; Red: $400 \mu \mathrm{m}$; Green:300 $\mu \mathrm{m}$.

Table 5.52. - Height values from focus, images after exposure to laser and corresponding Raman

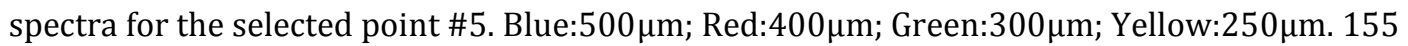

Table 5.53. - SNR values for the $405 \mathrm{~cm}^{-1}$ peak of hematite in all the RLS-ExoMars-like previous spectra. Corresponding height from focus, spot size at that height and irradiance is also presented in it. Green cells show the best SNR values for each point. 
Table 5.54. - Height values from focus, images after exposure to laser and corresponding Raman

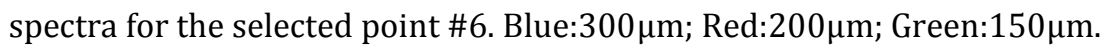

Table 5.55. - Height values from focus, images after exposure to laser and corresponding Raman

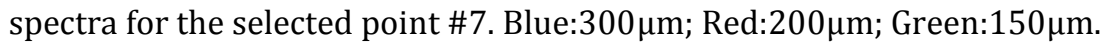

Table 5.56. - Height values from focus, images after exposure to laser and corresponding Raman

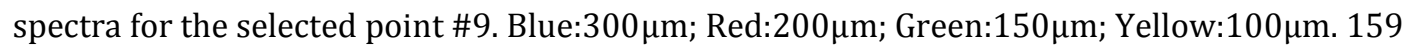

Table 5.57. - Height values from focus, images after exposure to laser and corresponding Raman

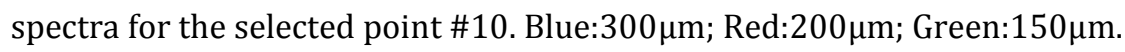

Table 5.58. - SNR values for the $405 \mathrm{~cm}^{-1}$ peak of hematite in all the Earth-like previous spectra.

Associated height from focus, spot size at that height and irradiance is also presented in it.

Cells in green mark the best SNR values for each point. 



\section{Chapter 1}

\section{Introduction}

Mars gained media relevance on the second half of the $19^{\text {th }}$ century derived from certain astronomical observations, misinterpretation of the data and science fiction works that appeared to raise the popular interest in our neighbour planet. This relevance translated into scientific curiosity during the $20^{\text {th }}$ century, when the technology made it possible to reach the planet and its surroundings by means of orbiters and landers.

The main questions regarding Mars relate to life and its origin. Mars could have once harboured the environmental conditions to develop and sustain life. The new science of Astrobiology seeks to find answers to these mysteries by applying the knowledge from natural sciences and the tools that engineering puts to their service. The Viking landers, the MER rovers, MSL and ExoMars were, are, and will be instruments of paramount importance to progressively approach to the questions of where we come from or if we are alone in the Universe.

Raman spectroscopy, materialized through the Raman Laser Spectrometer (RLS) instrument of ExoMars, will provide a rapid chemical analysis of the samples extracted from the subsurface of the planet. This powerful technique could have the key to the Holy Grail of astrobiology. 



\subsection{Planetary Exploration of Mars}

The astronomical observations of Mars made by Giovanni Schiaparelli (1835-1910) since the 'Great Opposition' of the planet in 1877, exposed a series of features on the surface of Mars: the canali or 'channels' in its translation to English (Figure 1.1).

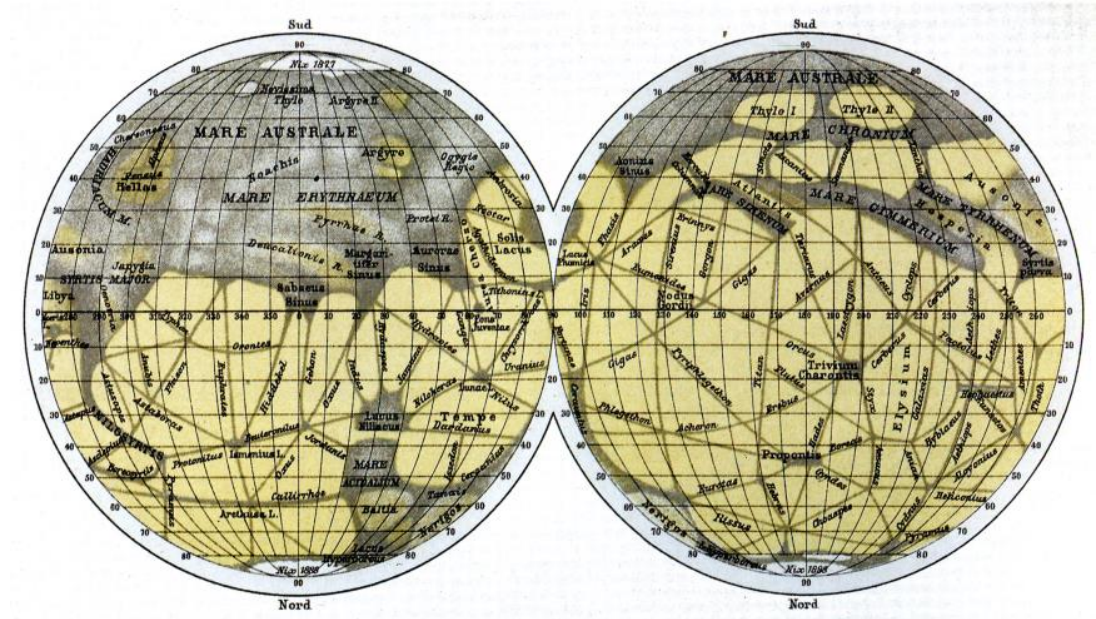

Figure 1.1. - Map of the planet Mars (1877-1888) by Giovanni Schiaparelli (Credit: International Planetary Cartography Database)

The mistranslation of this canali into 'canals', gave to the term an artificial connotation that the American astronomer Percival Lowell (1855-1916), among others, associated with intelligently built structures evidencing the presence of an extraterrestrial civilization inhabiting our neighbour planet (Figure 1.2).

With "The War of the Worlds" (1898) by H.G. Wells (Figure 1.3a) and its radio broadcast on CBS (1938) by Orson Welles, a high popular enthusiasm in Mars and the possibility of this planet to harbour not only life, but also intelligent life settled in the world's minds.

These events probably inspired Edgar Rice Burroughs (1875-1950) and Ray Douglas Bradbury (1920-2012) to write the sci-fi Barsoom series (Figure 1.3b) and The Martian Chronicles (Figure 1.3c), respectively, both of them set in Mars. 


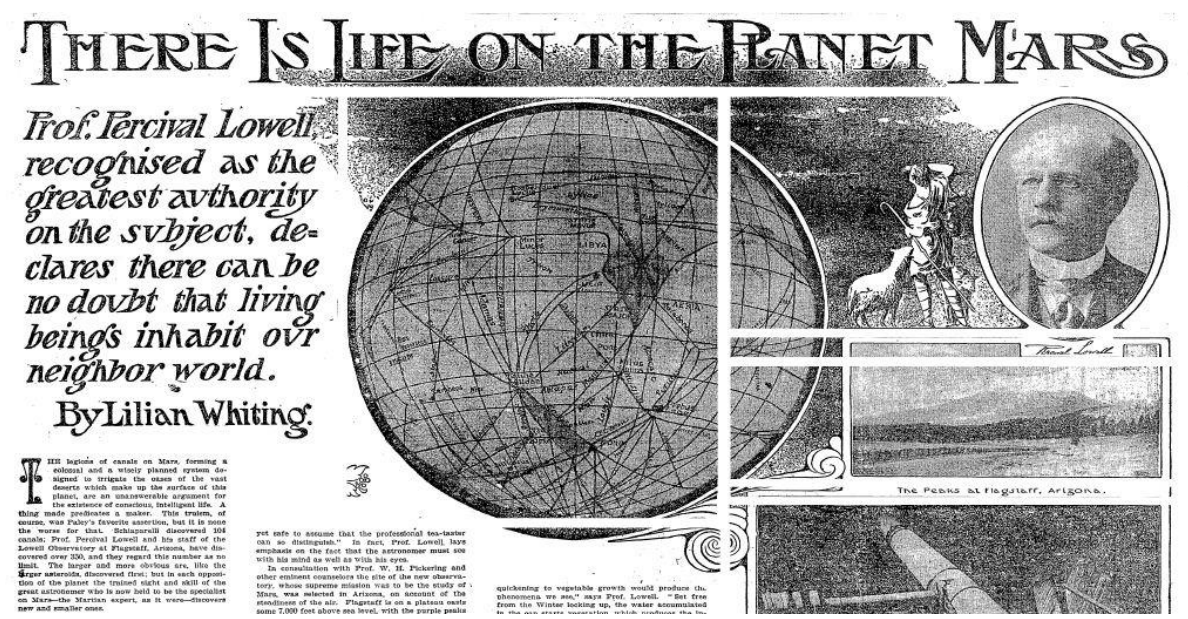

Figure 1.2. - Misinterpretation of Schiaparelli's 'channels' by Percival Lowell (Credit: The New York Times, December 9, 1906)

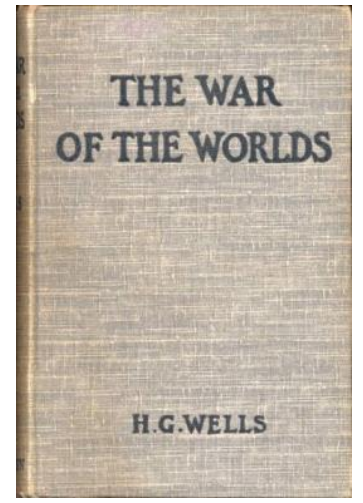

a

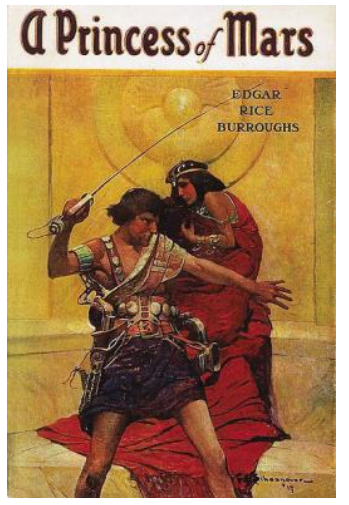

b

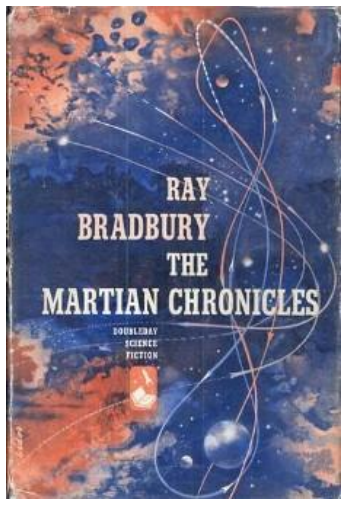

C

Figure 1.3. - Three science fiction books related to Mars's intelligent beings. (a) The War of The World by H.G.Wells (public domain image), (b) A Princess of Mars, the first book of the Barsoom series by Edgar Rice Burroughs (public domain image), and (c) The Martian Chronicles by Ray Bradbury (Credit: Doubleday publishing company)

And, as usual, when technology has matured enough, sci-fi and science shake hands...

In the second half of the $20^{\text {th }}$ century, the Mariner Space Program was the first set to explore the inner planets of our Solar System. Among them, Mars and its mysteries. The pioneers Mariner 4 (launched in 1964), 6 and 7 (both launched in 1969) provided images (Figure 1.4) and physical information of Mars and its atmosphere. They revealed a hostile environment and no traces of intelligent beings on the surface of Mars. This scenario set a different scientific approach to search for evidence of life on Mars. 


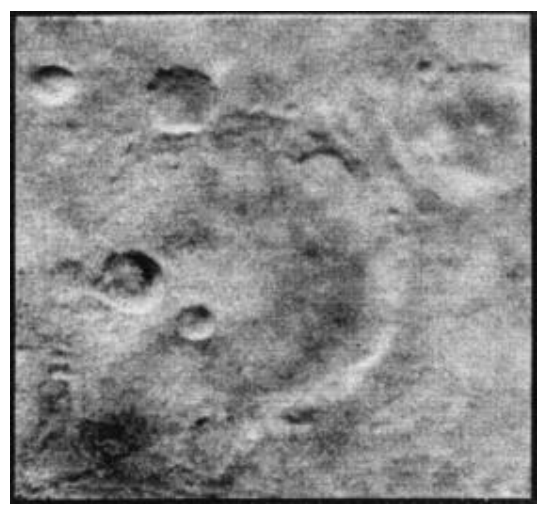

a

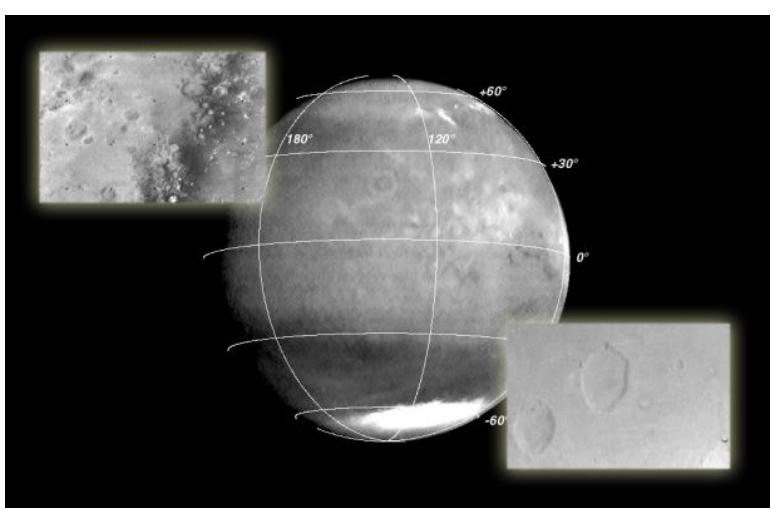

b

Figure 1.4. - Images of the Mars surface by (a) Mariner 4 (Credit: NASA) and (b) Mariner 6 and 7 (Credit: NASA)

The Viking Program (1975) took this lead with the touch down on Mars (Figure 1.5) of two landers with scientific payload designed to determine the presence or absence of biosignatures in the Martian soil. Until these days the results obtained by the scientific instruments on board the Viking probes remain inconclusive (Klein, 1999).

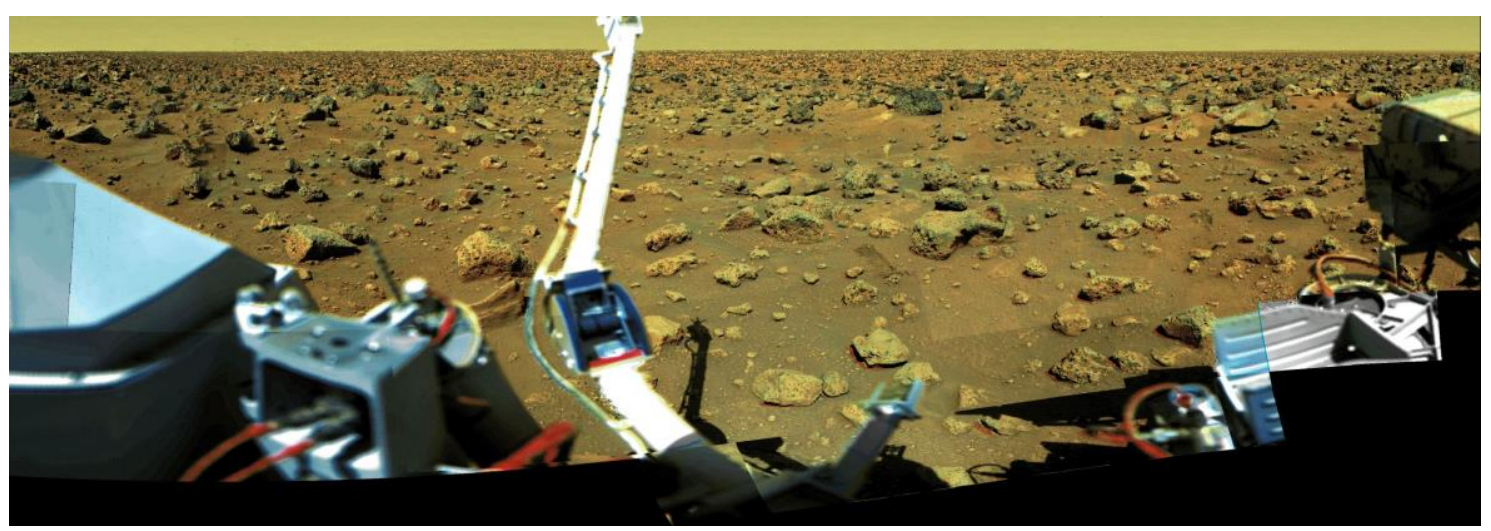

Figure 1.5. - Viking 2 panorama taken at its landing site (Credit: Edward A. Guinness, Washington University in St. Louis)

But the search for answers regarding life on Mars did not end up here and the Mars Pathfinder (1996) with its small rover Sojourner (Figure 1.6) put a new ability on the Mars surface: mobility. The mission found at the landing site pebbles that could indicate that an ancient flowing liquid shaped the surface of the region (Matijevic et al., 1997). This characteristic was also observed from orbit with Mars Global Surveyor (1996), Mars Express (2003) and Mars Reconnaissance Orbiter (2005). 


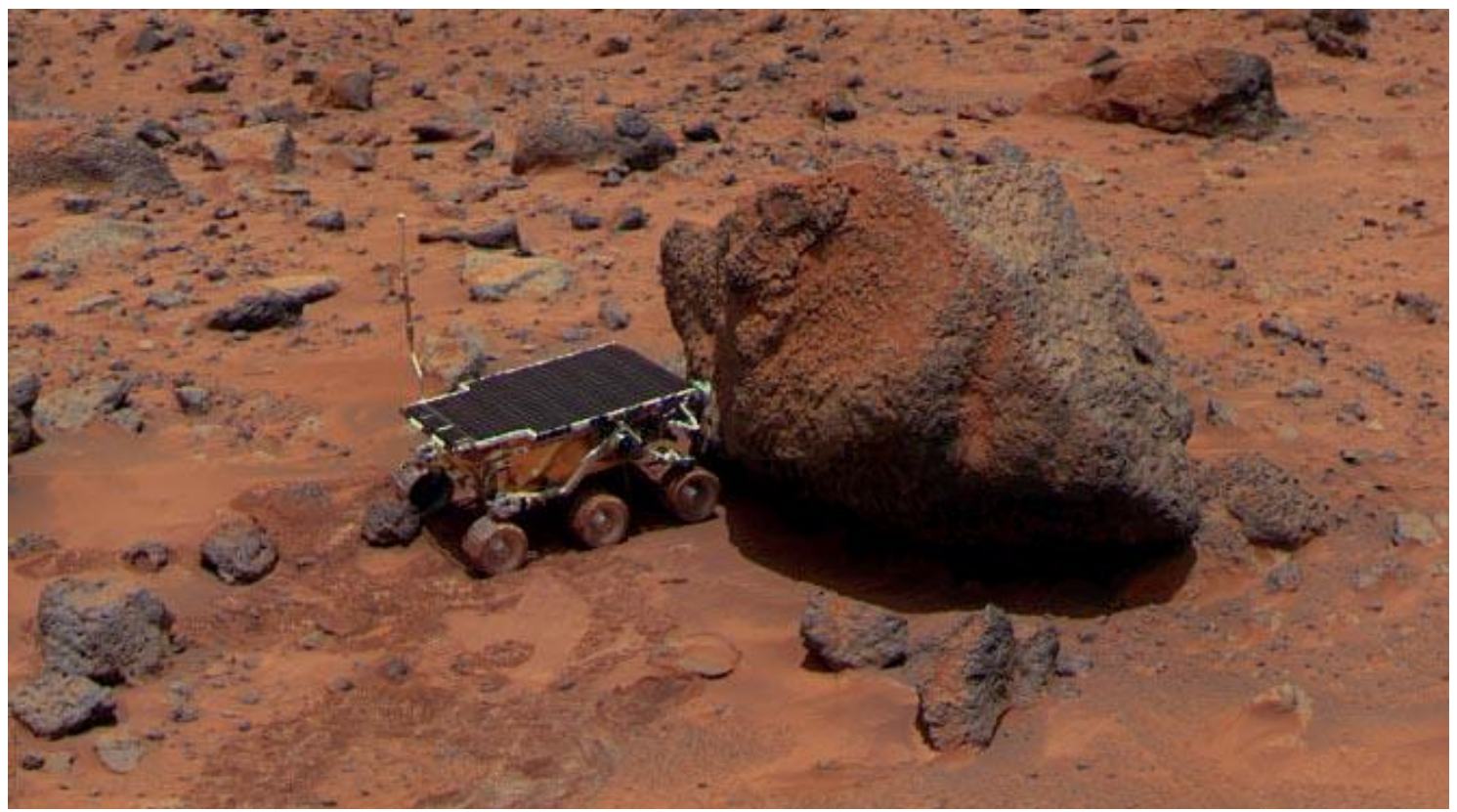

Figure 1.6. - Sojourner rover analyzing Yogi rock (Credit: IMP Team, JPL, NASA)

If liquid water run across the surface of the planet sometime in the geological past of Mars, it should have left mineralogical fingerprints on the surface of the planet. NASA's Mars Exploration Rovers (MER), Spirit and Opportunity, were landed on Mars (2004) to 'Follow the water'. And they did. They found carbonates (Morris et al., 2010) (Figure 1.7), sulphates and oxides (Klingelhöfer et al., 2004), mineralogical evidences of a wetter past (Bibring et al., 2006).

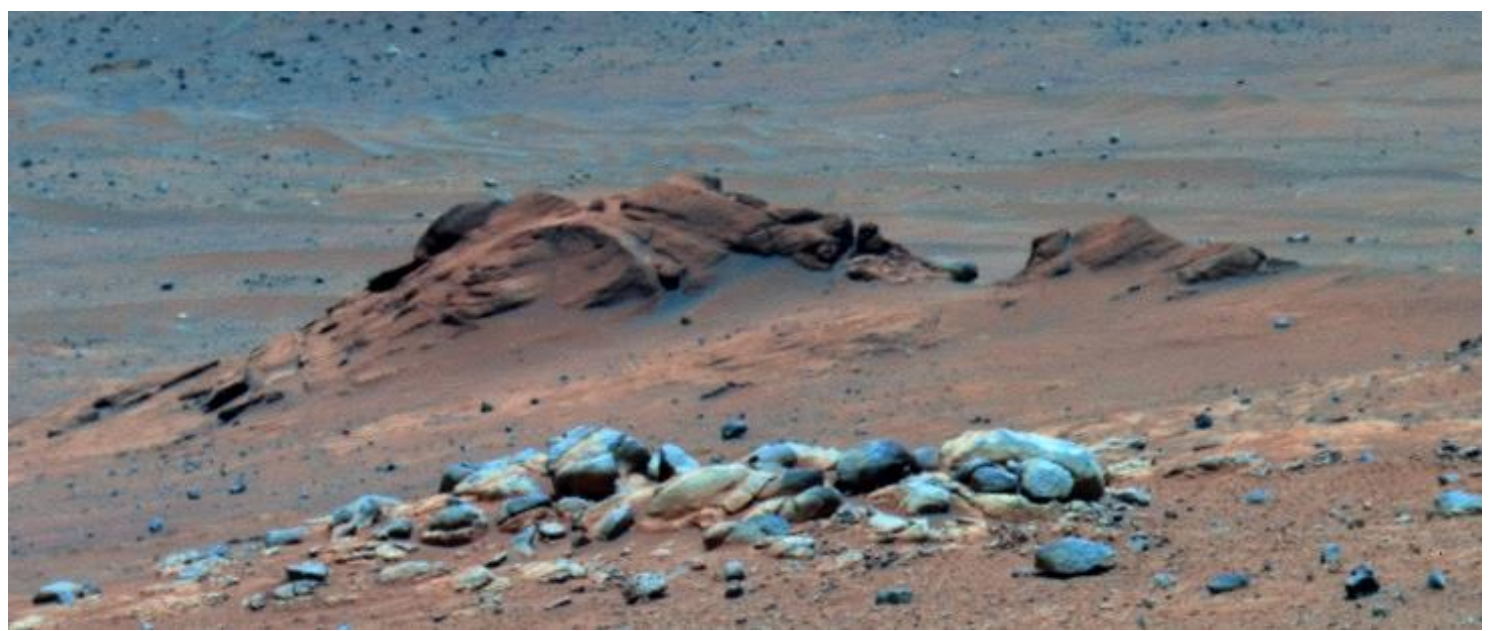

Figure 1.7. - Comanche outcrop (at the back) found by the Spirit rover on Mars. It is composed, in part, of Fe-Mg carbonates. (Credit: NASA/JPLCaltech/Cornell University)

A geologic period of time where water was stable on the surface of our neighbour planet could indicate that life could have arisen and evolved on the 
surface of Mars. Finding life on another planet apart from ours could be the most relevant discovery of all times, or at least the one with more scientific and philosophical implications.

To answer these, NASA's Mars Science Laboratory (MSL) is now roving Mars with the objective to find habitable niches on the surface of an ancient flooded crater, Gale Crater (Figure 1.8).

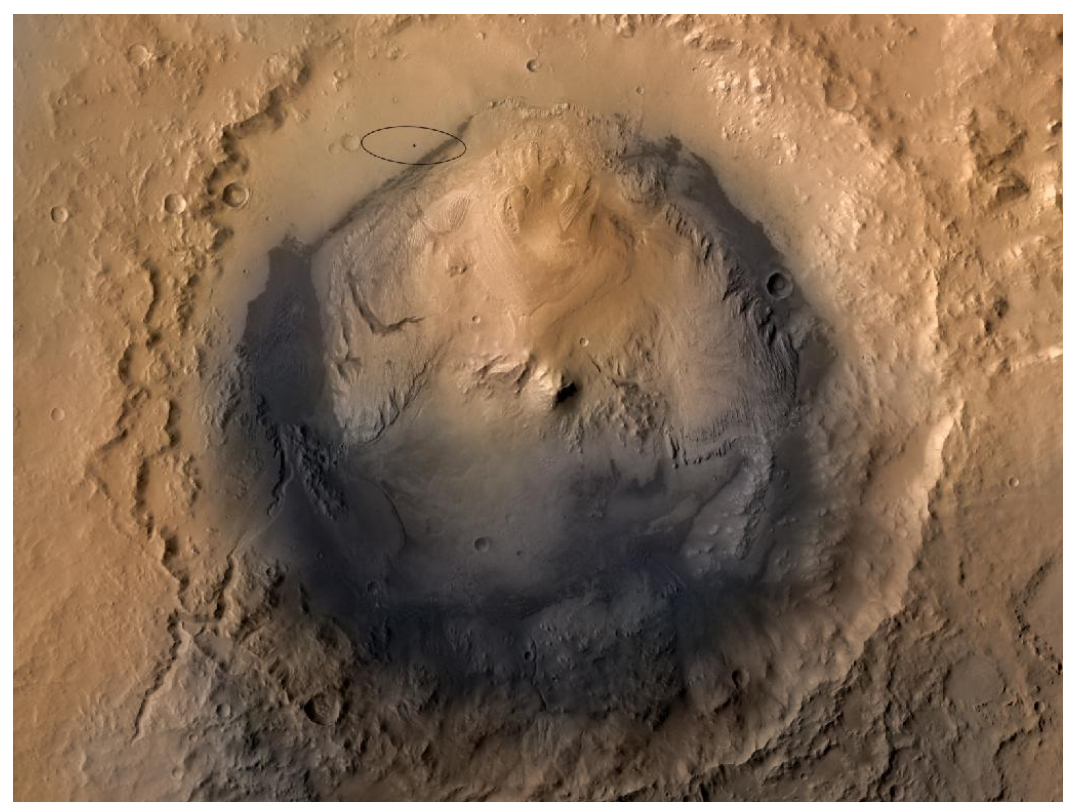

Figure 1.8. - Gale crater and the landing elipse for MSL. Gale crater is a layered mound of clays and sulphates. (Credit: NASA / JPL-Caltech / ESA / DLR / FU Berlin / MSSS)

Future missions, such as ESA's ExoMars, will go even one step further, searching for life itself, whether extant or extinct. ExoMars will be presented later in this chapter. 


\subsection{Astrobiology and Life}

Where do we come from? What is the origin of life? Did it begin on Earth or did it come from outer space? Does life exist beyond our planet? Is it common or scarce? These and many others are the greatest questions that surround the scientific issue of life.

To answer them all, a new science was born: Astrobiology, also known as Exobiology, seeks to find the answers to the origin, evolution and distribution of life in the Universe. This transdisciplinary science puts chemistry, astrophysics, biology, geology and even engineering to the service of the research around life: its meaning, structure, ingredients and mechanisms for its creation and development, its origin and/or evolution on Earth, limits to its survival and if it exists or ever existed in other regions of our Universe.

Life is a difficult term to define and every discipline has its own words to express a definition to it (Luisi, 1998), but the most elegant scientific definition for life is:

"A self-sustained chemical system capable of undergoing Darwinian evolution" (Joyce et al., 1994)

How life began is still an unsolved mystery. The issue of whether life is an inevitable consequence derived from organic chemistry under the right environmental conditions or an extremely fortunate accident is an open question to these days. What we do know is that life, as we know it, needs a series of ingredients to develop, among which liquid water, acting as a solvent for organic compounds and a diffusion medium, is one of the most important. Finding liquid water does not guarantee the existence of life, but is key to its discovery.

For liquid water to exist on a planetary surface, this rocky body has to be orbiting its parent star at such a distance that, in combination with a planetary atmosphere, provides the correct range of temperature and pressure conditions for it to exist. It has to be orbiting inside what has been defined as its habitable zone (Kasting et al., 1993). 
Once that condition is accomplished, for liquid water to sustain life it has to be rich in reduced organic molecules and $\mathrm{C}, \mathrm{H}, \mathrm{O}, \mathrm{N}, \mathrm{P}$ and $\mathrm{S}$ atoms, the building blocks of complex organic molecules that comprise life.

But, where do we get them from? Do they form onsite from inorganic or simple organic molecules? Do they arrive from outer space as water does during the accretion of planetesimals in planetary formation?

Complex organic molecules and amino acids have been detected out there in the Universe, in the largest molecular clouds of our Galaxy and inside the smallest bodies of our cosmic neighbourhood (Ehrenfreund \& Charnley, 2000). These could be the "alien" sources for organics, to add up to the inner production in our host planet. Going to the extreme in this transfer of life ingredients among planetary bodies, panspermia advocates for the transfer of life itself (Burchell, 2004). Astrobiology also studies the survival of life in extreme environments, such as those of interplanetary space.

So, once a planetary body contains liquid water with prebiotic organic molecules, how is life assembled from them? How does life contain itself inside the boundaries of membranes? Explanations go from the vesicle-forming fatty acids found in the Murchinson meteorite (Deamer, 1985), the iron-sulfur world (Wächtershäuser, 2006), the clay theory, to that of the RNA-world (Gilbert, 1986).

The most primitive form of life that, to this moment, scientists have been able to identify on Earth are the cyanobacteria, with the stromatolites as their quintessential fossil remnants. Recent scientific articles have found strong evidence of stromatolites dating $3.45 \mathrm{Ga}$ (1.15 Ga after the formation of the Earth) at the Pilbara Craton (Australia), one of the few geologic formations of the planet dating back to the Archean Eon (3.8 - 2.5 Ga) (Allwood et al., 2006, 2009).

During the Archean, the Earth was a huge magmatic sphere whose heat was maintained by the accretion of planetesimals, the core formation and the decay of radioactive elements. Whether at that time there was or wasn't plate tectonics processes is something under scientific discussion. If any, the recycling rate of the lithosphere was so high that it prevented the formation of early continents. Having 
the cooling and solidification of the Earth's crust already started (or not), this would have been re-melted by the Late Heavy Bombardment (4.1 - 3.8 Ga), a period of high rate of meteoritic impacts that transformed their gravitational energy into heat. The Late Heavy Bombardment is (part of) the reason why geological outcrops from the Hadean are not preserved. After the (re)cooling of the Earth's mantle and crust, the first continents emerged and archaic cratons found today in certain geographic areas are the result of this process.

The geological history of Mars is somewhat different. Because of its smaller volume, geological differentiation and subsequent cooling occurred during the first tens of millions of years since the planet's formation. The Late Heavy Bombardment (Carr \& Head III, 2010), in this case, contributed to the formation of large basins and craters, and perhaps to the North-South dichotomy (Figure 1.9) too (there's an open debate on whether the thinning of the crust of the northern hemisphere was caused by direct impact or due endogenic causes) (McGill \& Squyres, 1991). This cooling and no evidence of plate tectonics (Nimmo \& Tanaka, 2005), provide a geological time travel greater than that that can be performed on our planet. 

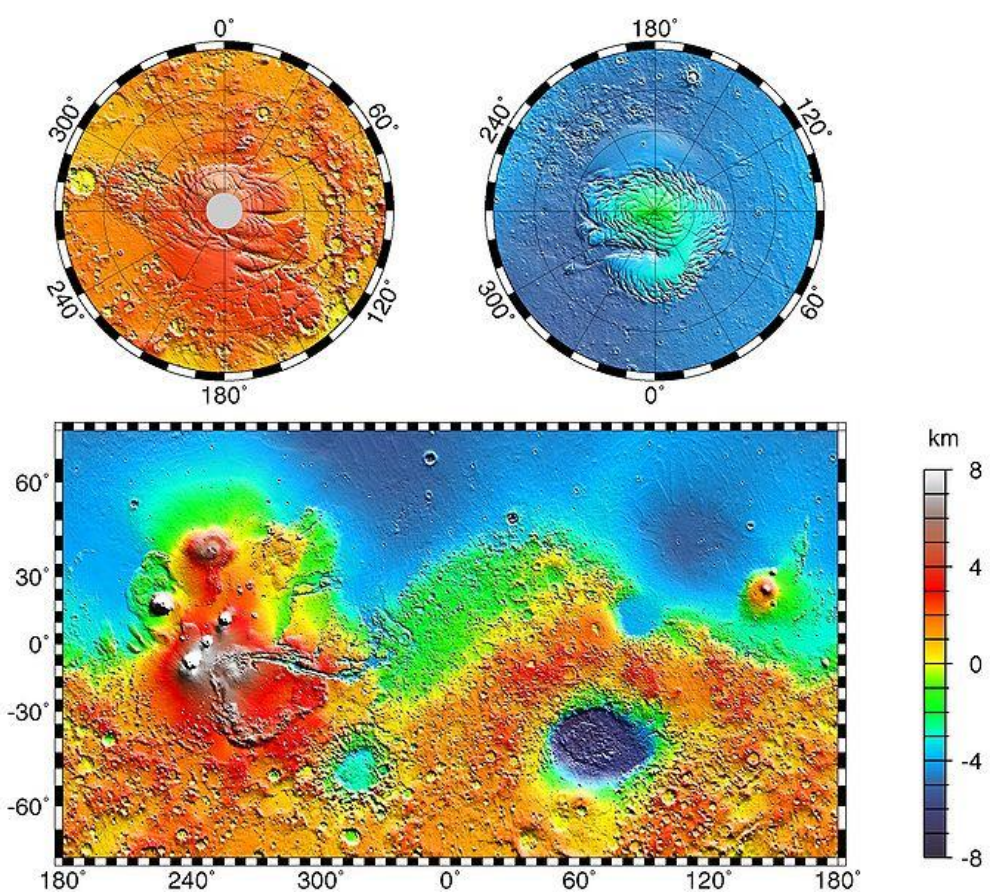

Figure 1.9. - Mars global map. A clear difference in height can be observed between smooth northern highlands and the rugged southern lowlands. (Credit: MOLA Science Team)

If life appeared on a watery past of Mars, long before the Late Heavy Bombardment, the traces of this event that had survived the meteoritic impacts and aeolian erosion would be kept intact in geologically protected places. The existence of these tracks could account for:

The origin of life on Earth, carried by Martian meteorites ejected from the surface of this planet by impacts.

Another origin of life, independent of that of Earth.

Another type of life, not carbon-based.

The hypothesis of possible propitious conditions for the habitability of early Mars is motivated by the study of the controversial ALH84001 Martian meteorite (3.9 - 4.0 Ga) (Halevy et al., 2011) and its apparent (McKay et al., 1996), but of dubious evidence (Martel et al., 2012), biogenic microstructures (Figure 1.10), and the in situ discovery, by NASA's rover Spirit of carbonate outcrops in Columbia Hills, Gusev crater (Morris et al., 2010). 


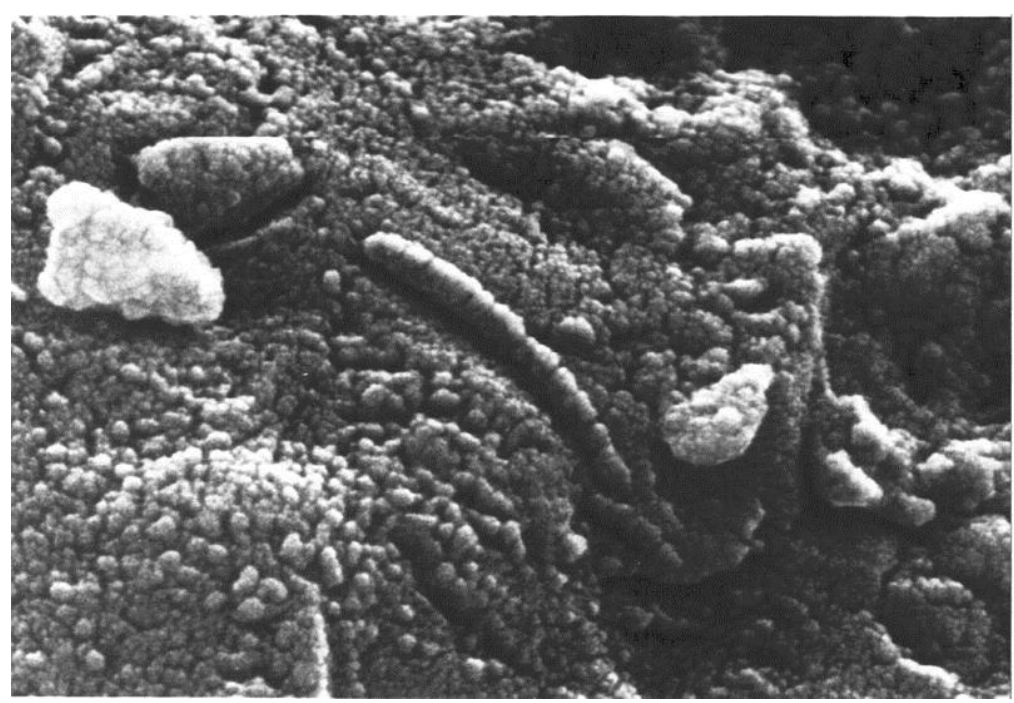

Figure 1.10. - SEM image for ALH84001 interpreted biogenic microstructures (Credit: NASA)

The existence of life on Mars is also an open question, motivated by the uncomfortable (and far from being solved) results of the life detection experiments aboard the Viking landers in the 70's (Levin \& Straat, 1976; Ponnamperuma et al., 1977; Yen et al., 2000; Bianciardi et al., 2012).

As it has been said, for life as we know it to currently exist on Mars (or anywhere else in the Universe), the main requirement is the existence of liquid water. Today there may be liquid water on Mars in some areas and at certain times of the year, especially for briny solutions (Haberle et al., 2001), but no large stationary masses of water where life could be thriving.

As the environmental conditions on the Mars surface became harsh to liquid water and to potential life, both could have migrated inwards in the planet's subsurface (Ehlmann et al., 2011), to places protected from lower pressures, radiation and strong chemicals. And that is where ESA's ExoMars is going to look for signs of life, in the shallow subsurface. 


\subsection{The ExoMars Programme and the 2018 ExoMars Rover}

There are two ways of clearing out doubts regarding if life flourished on Mars and if it is still there: expect to find new pieces of Mars that have fallen on the surface of our planet or go to Mars after them.

The ExoMars Programme (Vago et al., 2006), a European Space Agency (ESA) lead mission to Mars, aims to advance in the search for the answer to many of the questions that relate life and Mars. The ExoMars Programme (2016-2018) is comprised of two missions that will be launched in 2016 and 2018, respectively (Figure 1.11).

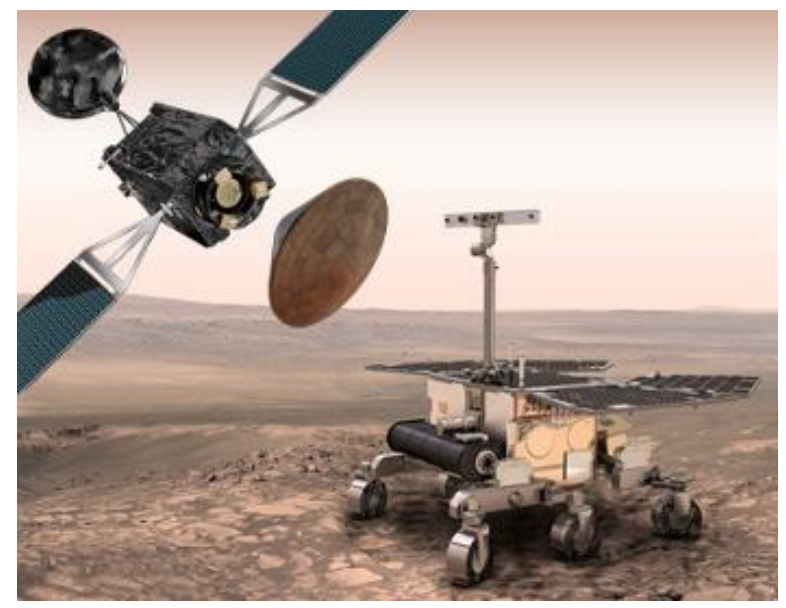

Figure 1.11. - Elements of the ExoMars Programme: the Trace Gas Orbiter (TG0) on the upper left and the ExoMars Rover on the lower right. (Credit: ESA)

The Trace Gas Orbiter (TG0), to be launched in 2016, will not only serve as a communication relay for the 2018 mission but also will study the Martian atmosphere and its minor gases (concentrations $<1 \%$ ), which could have relation to geological and biological activities on the surface or subsurface of the planet. At the same time, it will localize the spots on the surface where these gases come from. These places could be firm candidates as landing sites for subsequent surface explorers.

This 2016 mission will also incorporate an Entry, Descent and Landing Demonstrator Module (EDM), a landing capsule that will test the technologies needed to place a scientific payload on the surface of another planet. This is a 
pending subject of the European Space Programme, and it will be essential for the landing on the surface of Mars of the 2018 mission.

In 2018, ExoMars will deploy on the Martian surface an exploration rover capable of penetrating into the subsurface of the planet to retrieve samples from a depth of up to -2m (Figure 1.12). Its goal: look for signs of past and/or present life in places protected from the current hostile environmental conditions (ionizing radiation, oxidizing substances, low temperatures and low atmospheric pressure) on the surface of Mars.

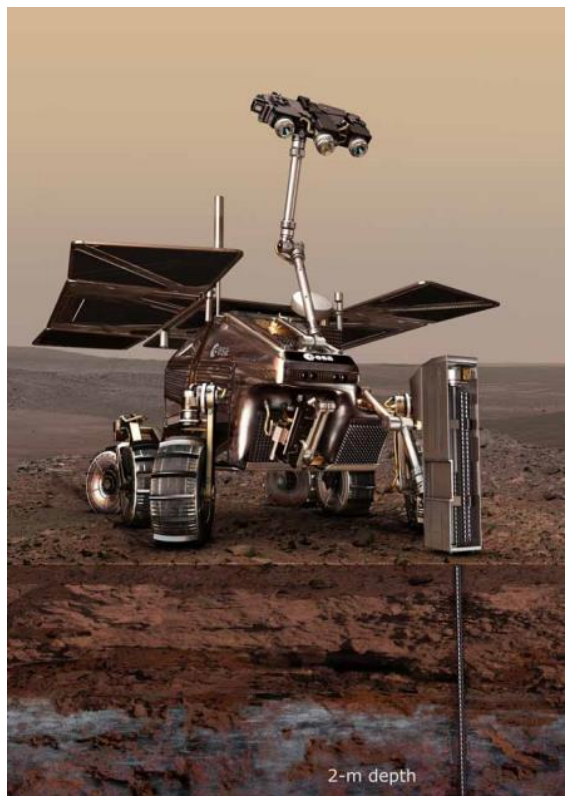

Figure 1.12. - Artist conception of the ExoMars rover drilling down to $-2 \mathrm{~m}$ in the subsurface of Mars. (Credit: ESA)

The ExoMars rover is well equipped with a battery of scientific instruments to both navigate along the planet selecting the best sites to extract samples from, and analyse those samples and their environment during their extraction and after preparing and dosing them for the instruments inside the Analytical Laboratory Drawer (ALD) inside the body of the rover. Among the latter, an IR spectral imager (MicrOmega), a Molecular Analyser (MOMA) and a Raman Spectrometer (RLS) will extract the chemical information of what are the samples comprised of.

The scientific objectives of the 2018 ExoMars mission are:

- Search for signs of past and present life on Mars; 
- Investigate how the water and geochemical environment varies;

All three internal instruments complement themselves and are capable of contributing to these two scientific objectives. MicrOmega will take images in the IR to gather mineralogical information and, perhaps, signatures of biomarkers from the samples extracted from the Martian subsurface. RLS will give molecular information on the chemical species, biological or non-biological, comprising those same samples. Finally, MOMA will heat the samples to target biomarkers with the help of a gas chromatograph and a mass spectrometer. Remember the GC-MS instrument aboard Viking?

The ExoMars rover will perform at least 2 vertical surveys extracting samples every $0.5 \mathrm{~m}$ to a maximum of $2 \mathrm{~m}$. It will do it thanks to a drill tool of $700 \mathrm{~mm}$ in length with an integrated multispectral imager (Ma_MISS), and 3 additional extension rods of $500 \mathrm{~mm}$ each. From each of the drillings, a $1 \mathrm{~cm} \mathrm{x} 3 \mathrm{~cm}$ cylindrical core sample will be obtained (Figure 1.13).

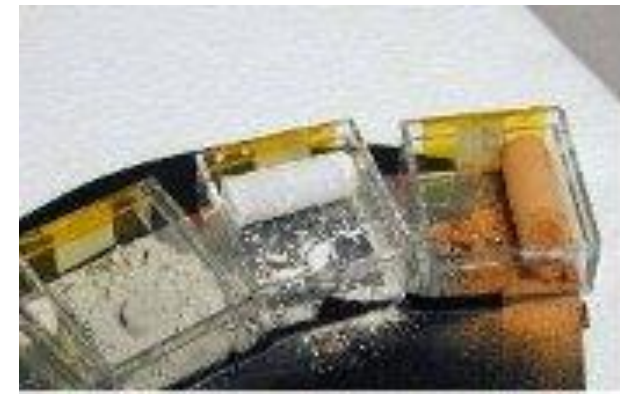

a

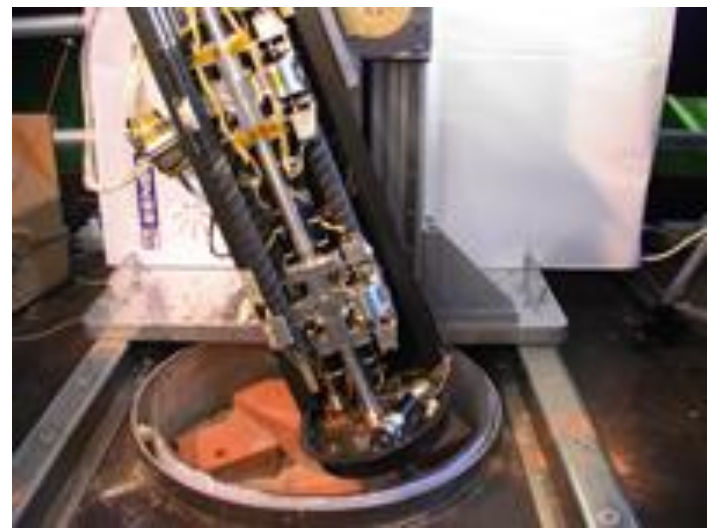

b

Figure 1.13. Examples of (a) core samples obtained with the (b) ExoMars drill unit. (Credit: ESA)

These core samples will be delivered to the Core Sample Handling System (CSHS), through the Core Sample Transportation Mechanism (CSTM), the interface part of the Sample Preparation and Distribution System (SPDS) with the exterior of the rover. The sample will then be passed to the Crushing Station (CS), where it will be converted to powder with a grain size distribution of Gaussian profile centred in $250 \mu \mathrm{m}$, with $90 \%$ of the grains in the range of diameters from $50 \mu \mathrm{m}$ to $500 \mu \mathrm{m}$. The Powder Sample Dosing and Distribution System (PSDDS, Figure 1.14) 
will be the responsible of depositing controlled amounts of sample onto the Refillable Container (RC). This RC, attached to the Powder Sample Handling System (PSHS), will move below the angularly distributed analytical probes of the instruments comprising the scientific payload after having been flattened by the Powder Sample Flattening Mechanism (PSFM). This operation will guarantee having $80 \%$ of the sample grains at a depth inferior to $200 \mu \mathrm{m}$ and $50 \%$ at less than $100 \mu \mathrm{m}$ from the surface of the RC. These distances will allow the instruments to have access to most of the sample.

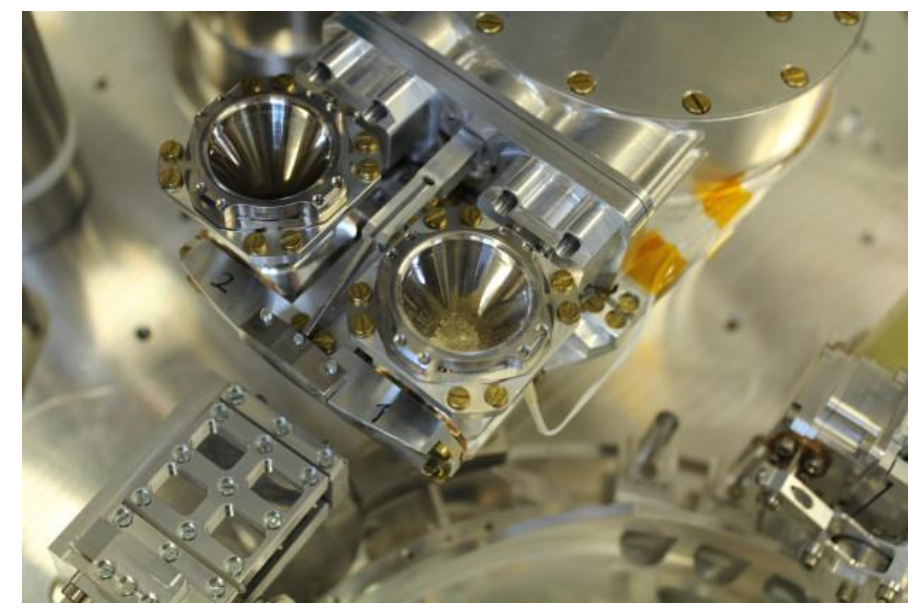

Figure 1.14. - The PSDDS loaded with sample. The RC can be seen just below it, on the PSHS. (Credit: Kayser-Threde GmbH.)

After every complete analysis of a sample, the Refillable Container Cleaning Mechanism (RCCM) will remove it out of the RC, to leave it ready for a new loading.

A mission like ExoMars is the next step in planetary exploration programs after the MER rovers, which corroborated that water once flowed on the Martian surface (Klingelhöfer et al., 2004; Morris et al., 2010), and MSL, which has found mineralogical signs that point to an ancient habitable environment at Yellowknife Bay, inside Gale crater (Grotzinger et al., 2014), once flooded, with a central mound of sedimentary rocks and a delta that accounts for this inflow of water.

The next step in the exploration of Mars after ExoMars, are sample return missions. These samples will be subjected to rigorous analyses in sophisticated terrestrial laboratories. If we want to better understand the geological and climatic past and if it ever supported life, and looking at the amounts of information 
extracted from the ALH84001 meteorite, bringing to Earth a selected piece of Mars will greatly help in this task.

\subsection{RLS-ExoMars and Raman spectroscopy}

The Raman Laser Spectrometer (RLS) (Rull \& Martínez-Frías, 2006) is an instrument selected to be part of the ExoMars's scientific payload. Its task will be to analyse the samples that the Sample Preparation and Distribution System (SPDS) provides to the instruments inside the rover, RLS being one of them.

The scientific objectives of RLS are directly inherited from those defined for ExoMars: Raman spectroscopy is able to extract the composition of the samples that the rover collects along its journey across Mars, from its surface and shallow subsurface, to (1) trace the geochemical history of the planet and (2) potentially identify organic molecules associated to biomarkers from extant or extinct Martian life forms.

Figure 1.15 shows the RLS instrument 3D layout presenting the three elements that it is comprised of:

- The internal Optical Head (iOH), which is mounted on top of the Ultra Clean Zone (UCZ) and looks directly into it through an optical window. The $\mathrm{iOH}$ has two optical fibre ports for the excitation and collection paths, and a communications port, which allows for the control of the autofocus (AF) mechanism. This AF allows the $\mathrm{iOH}$ to focus on every point along the sample over which it is placed. The $\mathrm{iOH}$ will project a $50 \mu \mathrm{m}$-diametre spot on the flattened sample for the Raman analysis of the chemical species contained in this area. A minimum number of 20 points per sample (TBC) along the accessible arc described by the movement of the RC on the PSHS will be analysed. The Raman light emitted by the excited sample is collected back with the $\mathrm{iOH}$ and directed to...

- The SPectrometer Unit (SPU), the Raman spectrometer itself, comprised of (1) the fibre connector, where the collection path ends, (2) the collimation optics for collimating the light onto (3) the transmission diffraction grating, 
(4) the imaging optics, and (5) the Charge-Coupled Device (CCD) imaging sensor. All the electronic and excitation units are driven by...

- The Instrument Control and Excitation Unit (ICEU), the electronics box with instrumentation for the actuation on the AF system, for achieving an accurate thermal control or with the Front End Electronics (FEE) for the CCD sensor commanding and signal processing, among others. The ICEU also hosts the 532nm (TBC) excitation laser, which connects to the excitation path through an optical fibre.

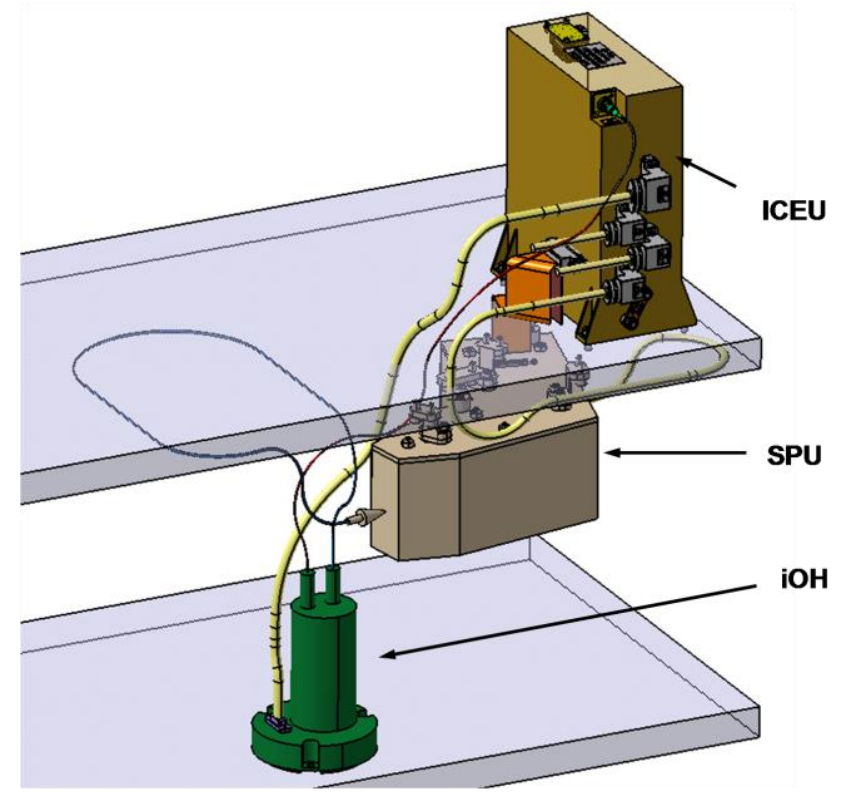

Figure 1.15. - RLS-ExoMars instrument layout with its three components: ICEU, SPU and iOH. (Credit: ESA)

Raman spectroscopy is a powerful analytical technique that allows the identification of materials from vibrations and rotations of their constituent molecules.

It works as follows: a laser beam (monochromatic light) is focused on the sample to be analysed. The laser photons interact with the electron cloud around the bonds of the molecules of the sample, exciting them to virtual energy states. When, after approximately $10^{-14} \mathrm{~s}$ the molecule is de-energized, it backscatters a photon whose energy is equal to the difference between the energies of the excited and final energy levels of the molecule. This energy may be higher or lower than the excitation energy depending on the energetic level to which it relaxes, thereby 
producing higher (Anti-Stokes) and lower (Stokes) frequency components measured from the emission frequency of the laser (Figure 1.16). Raman, unlike Rayleigh, is an inelastic scattering phenomenon, what means that the energies of the incident and backscattered photons are different. The Raman effect is also a weak one. For every $10^{8}$ photons scattered by the excitation source and reaching the sample, only one is scattered back as a Raman photon. The rest does as Rayleigh radiation.

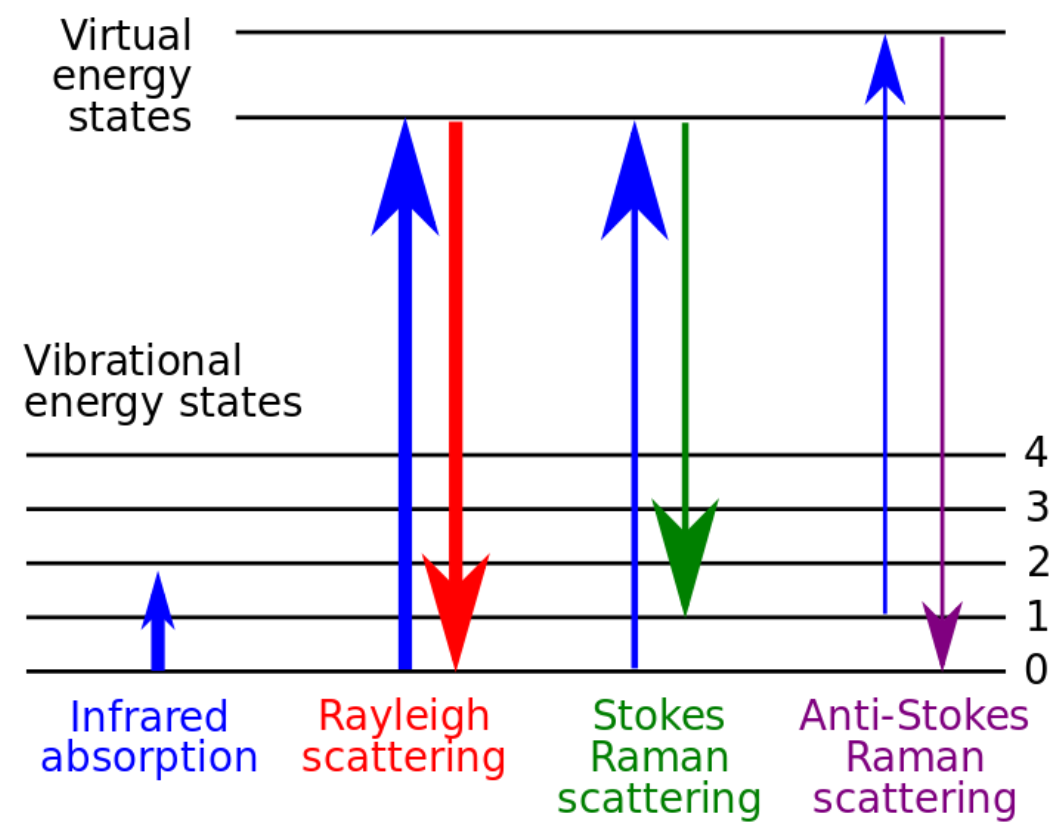

Figure 1.16. - Diagram of the energy levels and transitions for IR, Rayleigh elastic scattering and Raman Stokes and Anti-Stokes inelastic scattering. (Credit: Moxfyre / Wikipedia)

A Raman spectrum is the fingerprint resulting from dispersing the Raman light frequency components along an imaging sensor by means of a diffracting element and imaging optics. A Raman spectrum (Figure 1.17) is expressed in intensity versus wavenumbers (in $\mathrm{cm}^{-1}$ ) shifted from the excitation wavenumber or Raman shift. Each of the Raman peaks account for a vibrational/rotational mode of the molecules contained in the excited sample volume and provides information about the present molecular species (peak position), their degree of crystallization (peak FWHM) and concentration (peak intensity) in the sample, its temperature (ratio of Stokes-AntiStokes peak intensities), and many other characteristics. 


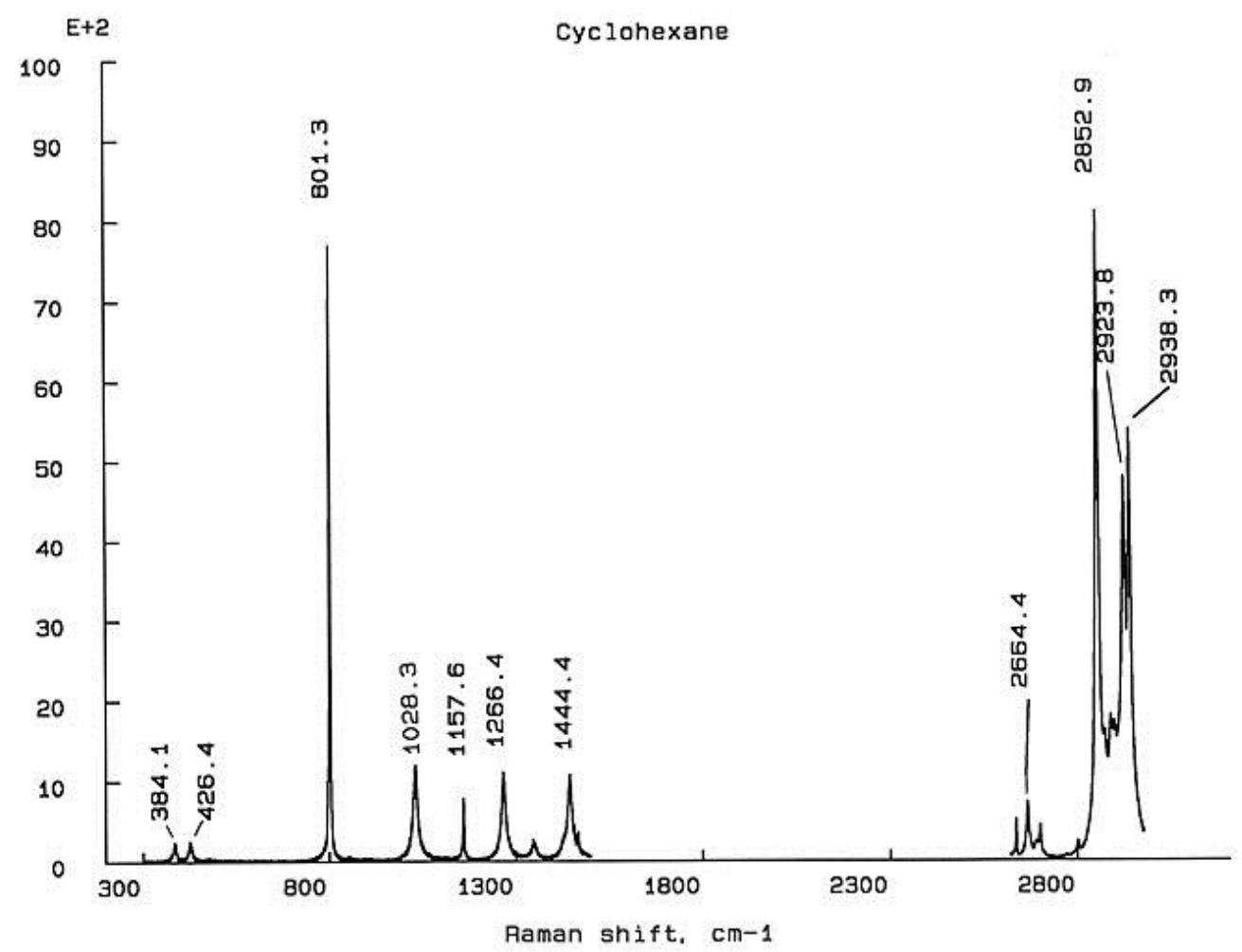

Figure 1.17. - Raman spectrum of Cyclohexane. (Credit: McCreery Research Group, National Institute for Nanotechnology, University of Alberta)

Two of the main advantages of Raman spectroscopy that makes it stand out over other analytical techniques are: (1) the sample requires little to no preparation at all, and (2) the time required for the analysis moves in the order of the seconds, or even milliseconds for very efficient samples. 


\section{Chapter 2}

\section{Justification and}

\section{Objectives}

This chapter explains the motivation for this $\mathrm{PhD}$ thesis and the objectives it

pursues. It also presents the structure of the rest of the manuscript for the readers' information. 

With the landing of the ExoMars rover on the surface of Mars the European Space Agency (ESA), Roscosmos and, extensively, the European scientific community and space industry will achieve an important milestone. Not only because of safely landing a scientific payload on the surface of another planet, something new for the Europeans, but also because it will be the first time that a Raman spectrometer lands on another planet as an instrument of a planetary exploration mission.

This important achievement brings an associated scientific necessity: much is known about the Raman spectral behaviour of all kinds of samples here on Earth. But there is much to learn about the Raman behaviour of these same samples under other planets' environmental conditions with special focus on samples of interest in the frame of planetary exploration such as hydrated samples, oxides and hydroxides because of their susceptibility of structural modification derived from both the new environment and the analytical technique.

Detecting and cataloguing the changes on these samples reflected in their Raman spectra helps establishing spectral databases under, in the frame of this thesis, RLS-ExoMars environmental conditions. These fine databases will serve as the basis to the scientific exploitation and interpretation of the spectral data received from the experiments carried out by the RLS instrument on Mars.

This need for scientific understanding and spectral classification sets the foundations for the development of new tools and instruments that provide with the ability to satisfy these requirements in order to accomplish the scientific objectives of the mission.

In the case of the ExoMars mission, the instrument needed not only has to reproduce the environmental conditions inside the zone for sample analysis in the ExoMars rover, but it also has to work with powder samples and flatten them before their analysis.

The RLS-ExoMars Simulation Chamber presented in this manuscript aims to meet these pressure, temperature, gas composition and sample flatness requirements to be able to perform scientific analyses of powder samples under 
the environmental conditions and sample specifications defined in the instrument and mission requirements for RLS-ExoMars.

Moreover, and attending to the operation mode defined for the RLSExoMars instrument, the further described RLS-ExoMars Science Simulator compliments the chamber in order to position the Raman Optical Head above any point of the sample to be analysed.

Having justified the need for the tool object of this PhD thesis, the structure of the next chapters of this manuscript is as follows:

Chapter 3 describes this instrument and all the systems and subsystems that comprise it, as well as the results of its testing.

Chapter 4 addresses the design and construction of an element that fits perfectly with the RLS-ExoMars Simulation Chamber. And that is the RLS-ExoMars Science Simulator, the optomechanical complement that puts in communication the samples inside the chamber and lasers and spectrometers outside it, to perform scientific experiments.

Chapter 5 deals with this last issue: the experiments. It presents two groups of experiments: a first one regarding the spectral differences that the relevant environmental conditions of the mission may impose on the Raman spectra leading to the need of creating a mission-specific database; and a second group of experiments centred in the study of the effects of the laser irradiance on two thermolabile samples with two different behaviours. This group of experiments were carried out under Earth lab conditions and also under RLS-ExoMars relevant conditions. This change of ambient highlights the contribution of a low-pressure atmosphere in terms of heat dissipation.

Chapter 6 summarizes the work done in this PhD Thesis, and reflected in this manuscript, and the main conclusions and outcomes.

Several annexes gather extra information related to the development of this thesis. 


\section{Chapter 3}

\section{RLS-ExoMars Simulation}

\section{Chamber}

The present chapter presents the next step in the scientific tools for testing the science around the ExoMars mission, particularly centred in the RLS instrument: an RLS-ExoMars Simulation Chamber.

This simulation chamber is intended to serve as a piece of Mars in the laboratory incorporating pressure, atmosphere and temperature variables, and a sample preparation subsystem. With it, the scientific team for RLS will be able to perform the science necessary to define, check and redefine the requirements for the flight instrument, as well as to test the operation mode. 



\subsection{Introduction}

Before the work presented in this PhD thesis, the ExoMars RLS Simulator (López-Reyes et al., 2013) could only perform its operations under laboratory conditions. This means that it is bounded to the temperature, humidity, atmospheric pressure and composition of the terrestrial environment in which it is enclosed at the Unidad Asociada UVa-CSIC-CAB 1.

The next step in the simulation of the scientific operations that the RLS instrument will perform on Mars consists of the incorporation of the environmental conditions under which the sample will be analyzed: Martian atmospheric pressure and gas composition, and also temperature of the $\mathrm{RC}$ at the PSHS. This important instrumental step will allow the scientific team of the RLS instrument to perform experiments under more realistic conditions and assess how they influence both the evolution of the powdered samples and the procedures for their analysis in terms of additional or reviewed scientific requirements for the instrument.

To achieve this objective, the RLS-ExoMars Simulation Chamber has been designed and built following the ExoMars specifications (Ball, 2013). Figure 3.1 presents the 3D layout and a picture of the actual chamber already in operation at the prototypes laboratory of the Unidad Asociada UVa-CSIC-CAB. Figure 3.2 shows the scheme of the elements of this chamber, for a better understanding.

\footnotetext{
${ }^{1}$ Unidad Asociada UVa-CSIC a través del Centro de Astrobiología (INTA), Avda. Francisco Vallés 8, E-47151 Boecillo, Valladolid, Spain
} 


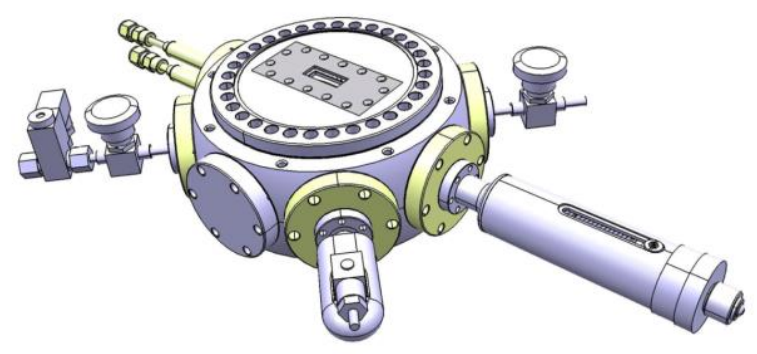

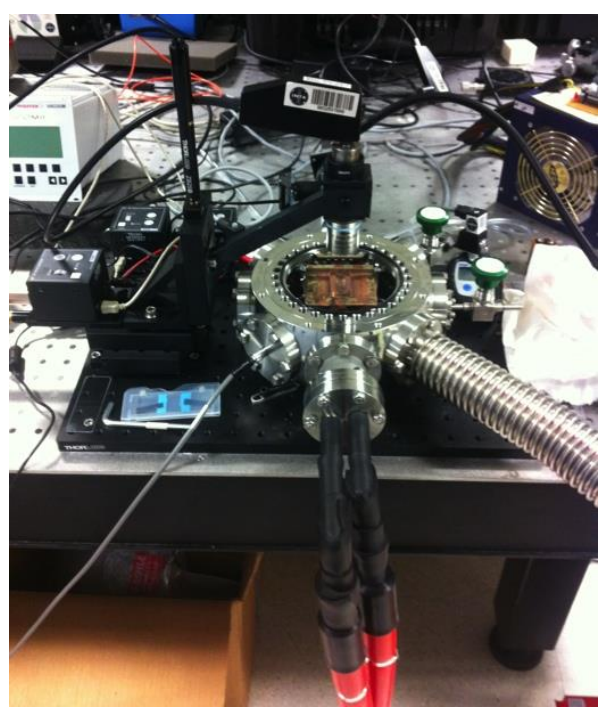

b

Figure 3.1. - (a) 3D layout and (b) real image of the RLS-ExoMars Simulation Chamber + RLS-ExoMars Science Simulator

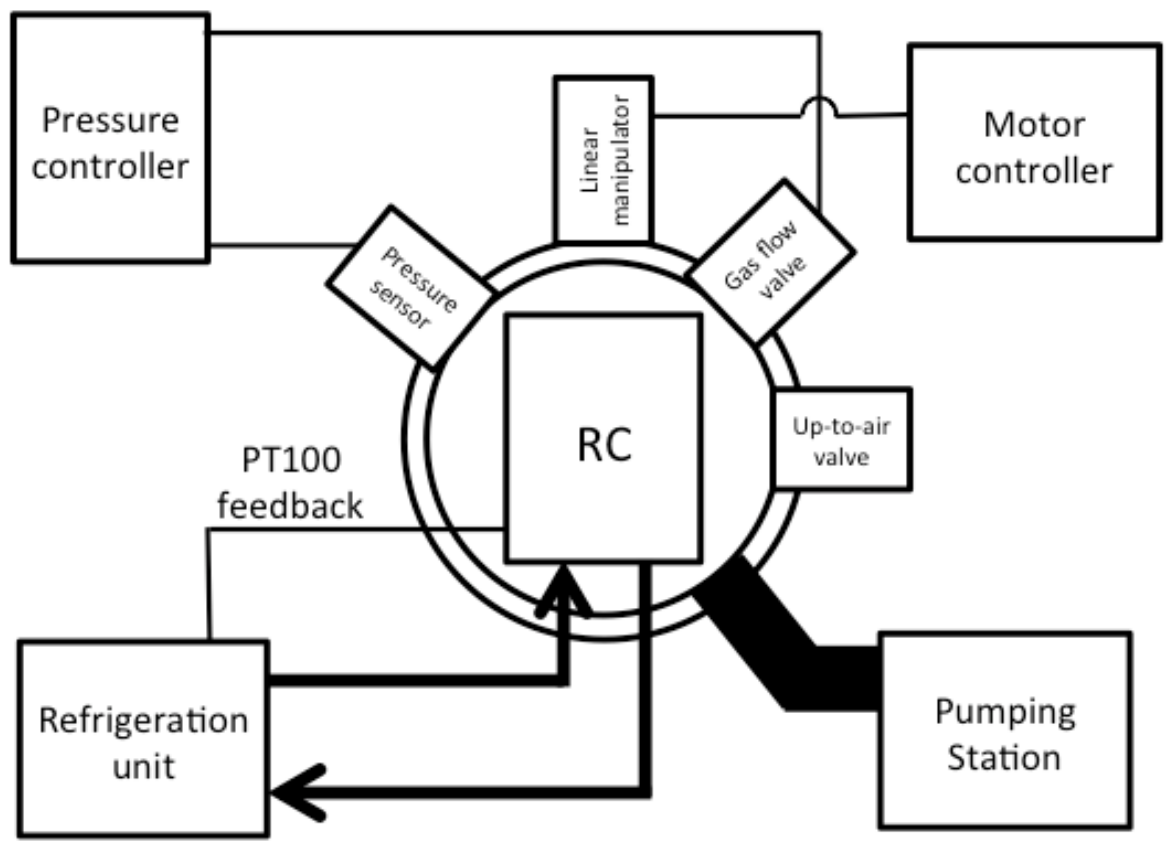

Figure 3.2. - Scheme of the elements of the RLS-ExoMars Simulation Chamber 


\subsection{Pressure and temperature systems}

The atmospheric pressure on the Mars surface varies from 0.3 mbar at the top of the Olympus Mons to the 11 mbar of the lowest point at the Hellas Planitia. This pressure varies with altitude, but also during a day, and between seasons. The average atmospheric pressure at the landing site of MSL at Gale Crater is around 7 mbar as measured by REMS. The atmospheric pressure at the landing site of ExoMars, yet to be decided, is not expected to vary that much from this value.

The atmosphere of Mars at its surface is comprised of $\mathrm{CO}_{2}$ in more than a 95\% (Owen et al., 1977).

The RLS-ExoMars Simulation Chamber presented in this chapter is capable of recreating inside it atmospheric pressures from $10^{-3}$ mbar up to 1000 mbar or Earth atmospheric conditions. For the atmospheric composition a $100 \% \mathrm{CO}_{2}$ atmosphere is used as an approximation of the real atmosphere.

Regarding temperature, Mars is a planet of extremes. As the atmospheric pressure on its surfaces is so low, less than 1\% that on Earth, the thermal inertia is very high and a rock on the surface of the planet can reach $20^{\circ} \mathrm{C}$ while its shadow is at $-50^{\circ} \mathrm{C}$. The temperature that matters to the purpose of the RLS operations is the one defined in (Ball, 2013). The requirement PPL-ENG-ALD-0180 establishes a temperature of $<-5^{\circ} \mathrm{C}$ for the sample during the scientific operations of RLS and MicrOmega instruments.

The RLS-ExoMars Simulation Chamber houses in its interior a Refillable Container (RC), which is connected to a liquid-refrigeration unit outside the chamber. This unit provides the temperature specified for the operations to the sample.

The next two subsections will present (in section 3.2.1) each of the parts of this pressure and temperature systems that make the establishment of the desired environmental conditions inside the simulation chamber a reality, and (in section 3.2.2) the tests performed to be sure the complete set reaches those conditions. 
Annex A presents the PC software specifically created to control this Pressure and Temperature Systems.

\subsubsection{Parts}

\subsubsection{RLS-ExoMars Simulation Chamber body}

The body of this simulation chamber is a 6" spherical octagon delivered by Kimball Physics, USA (Figure 3.3). The body is completely built in stainless steel.

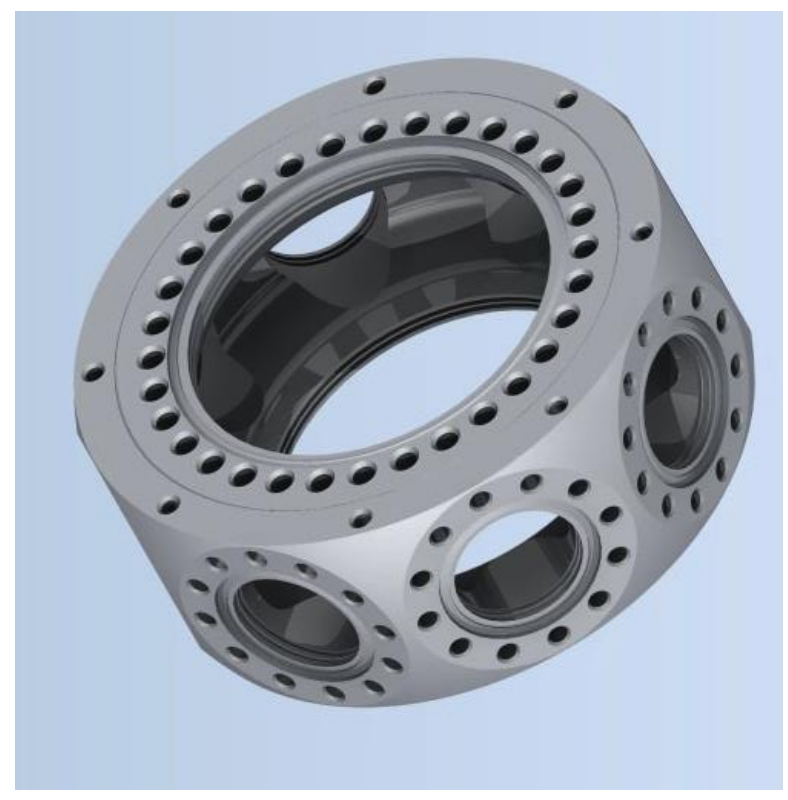

Figure 3.3. - 3D layout of the chamber body, a 6" spherical octagon. (Credit: Kimball Physics)

This element has two DN100CF ports at its top and bottom (as it is being used) and eight DN40CF ports angularly distributed around its perimeter. The bottom port is at the present permanently closed with a thin flange. To the top port a modified thin flange is used as a cover. This cover has been machined to host a rectangular fitting where an optical window is placed. This optical window will be presented later in section 3.3.1.3 as part of the sample system.

Although for the current design and operation the minimum attainable atmospheric pressure approaches $10^{-3}$ mbar, this chamber has been built thinking in future uses beyond the RLS-ExoMars mission. These vacuum ports theoretically allow for the achievement inside the chamber of Ultra High Vacuum (UHV), atmospheric pressures down to 10-12 mbar, useful in the simulation of the lunar surface for example, and above interplanetary space conditions. 


\subsubsection{Vacuum generation station}

For the achievement of the low atmospheric pressure conditions inside the RLS-ExoMars Simulation Chamber, a vacuum generation station is used. This equipment is a HiCube Eco 80 by Pfeiffer Vacuum (Figure 3.4) and it is comprised of a turbomolecular pump with a backup rotary pump. The station is capable of achieving UHV conditions inside the vacuum chamber to which it is connected. It has a screen and control buttons to manually actuate on the rotation speed of the blades of the turbomolecular pump, which affects to the end vacuum that can be reached.

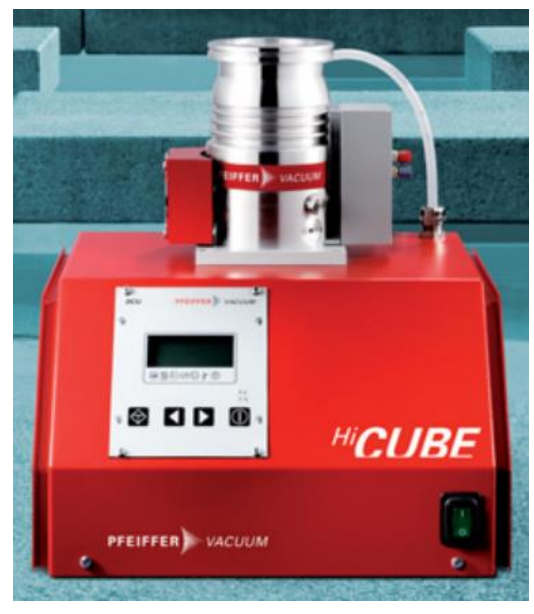

a

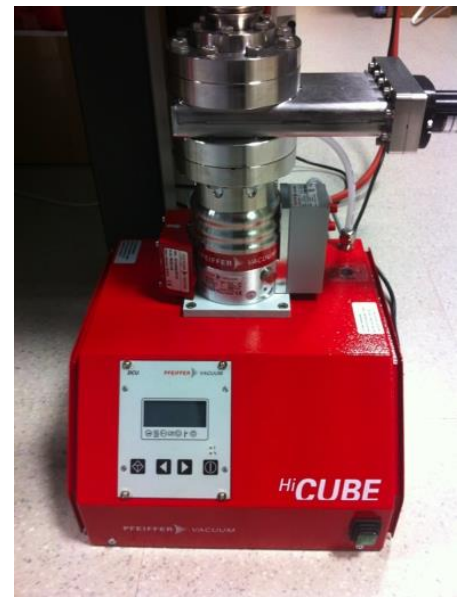

$\mathrm{b}$

Figure 3.4. - HiCube Eco 80 from (a) catalogue (Credit: Pfeiffer Vacuum) and (b) RLS-ExoMars Simulation Chamber assembly

Figure 3.4.b shows the vacuum station as it is installed in the prototypes lab for the RLS-ExoMars Simulation Chamber. On top of the turbomolecular pump a gate valve has been assembled, in case the chamber is to be closed for keeping a certain pressure, regardless of the vacuum station. Connected to the other side of this gate valve, there is a $1.5 \mathrm{~m}$-long stainless steel hose, which ends up in one of the lateral ports of the chamber (Figure 3.5). This is the feed line for the vacuum station. 


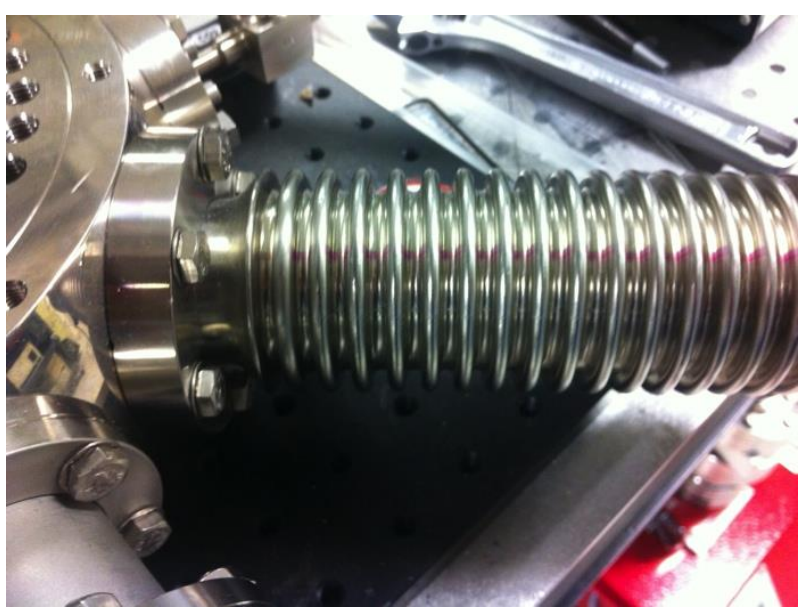

Figure 3.5. - Vacuum hose attached to one of the DN40CF lateral ports of the chamber body

During the experiments performed in this thesis, and presented in section 5, the duty cycle of the vacuum station was fixed at $26 \%$, providing a convenient pressure of $10^{-3}$ mbar from which to raise the final pressure to the Martian.

\subsubsection{Pressure sensor}

Knowing the gas pressure inside the RLS-Simulation Chamber is paramount for establishing the desired conditions. In this assembly the TPR280 Pirani sensor from Pfeiffer Vacuum was connected to another of the vacuum ports of the chamber (Figure 3.6).

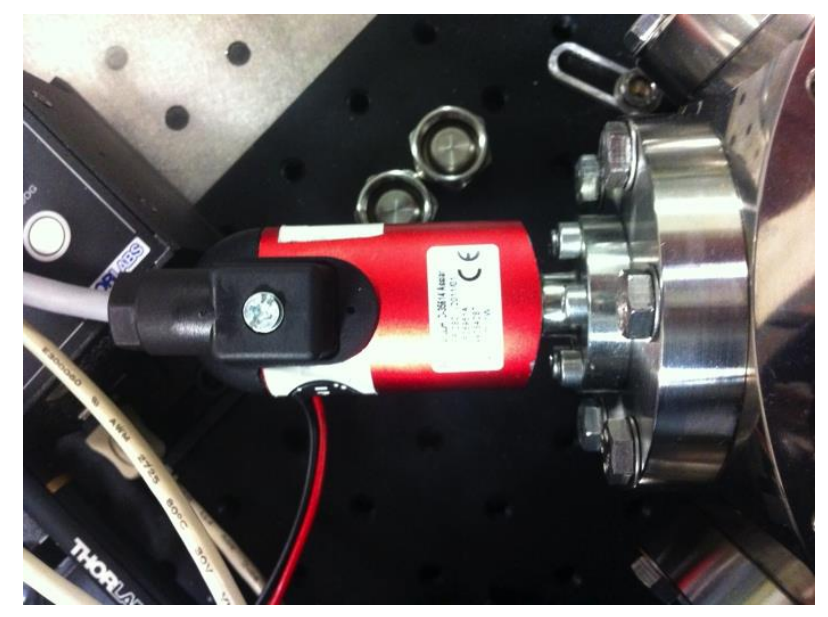

Figure 3.6. - TPR 280 Pirani sensor for the pressure measurement inside the RLS-ExoMars Simulation Chamber.

The TPR 280 is connected to a pressure controller, which will be presented in section 3.2.1.5. This kind of sensors are gas dependent, which means that the effective pressure inside the chamber has to be calculated from the pressure 
reading given by the pressure controller and a multiplication factor corresponding to the gas being injected to the vacuum chamber. This factor is extracted from the curves presented in Figure 3.7.

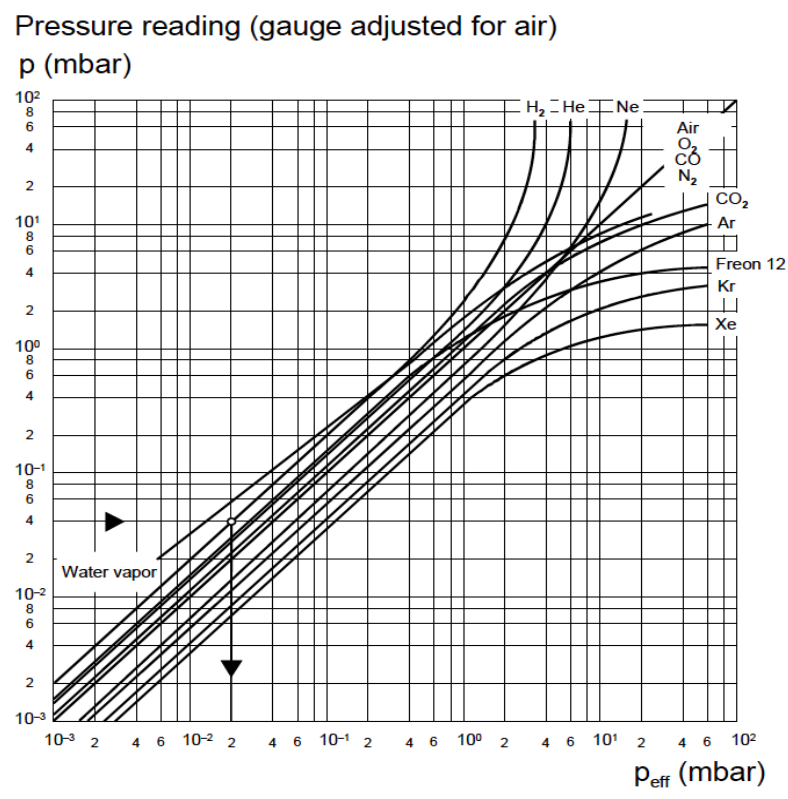

Figure 3.7. - Pressure curves for the TPR280 pressure sensor depending on the gas being used. (Credit: Pfeiffer Vacuum)

These curves are not tabulated so the value has to be extracted visually. In our case, $\mathrm{CO}_{2}$ and $~ 6 \mathrm{mbar}$, the effective pressure ( $\mathrm{p}_{\text {eff }}$ ) is 1.25 times the pressure reading.

\subsubsection{Gas and valves}

There are two manually actuated butterfly valves connected to the $R L S$ ExoMars Simulation Chamber. One of them is used to enable or disable the inflow of $\mathrm{CO}_{2}$ gas into the chamber and the other one is used to return to Earth lab atmospheric conditions after the experiments have been performed.

The first butterfly valve is usually left open and it is connected in series with an electroactuated gas flow valve, which regulates the flow of gas entering the chamber as commanded by the pressure controller, to be presented in the next subsection. This gas flow valve feeds from a rubber tube that transports $\mathrm{CO}_{2}$ from a gas bottle installed at a corner of the laboratory (see Figure 3.8). The whole valve system is presented in Figure 3.9. The small instrument placed just below the 
electroactuated gas flow valve in Figure 3.9.b is a $\mathrm{CO}_{2}$ sensor, used for obvious security reasons.

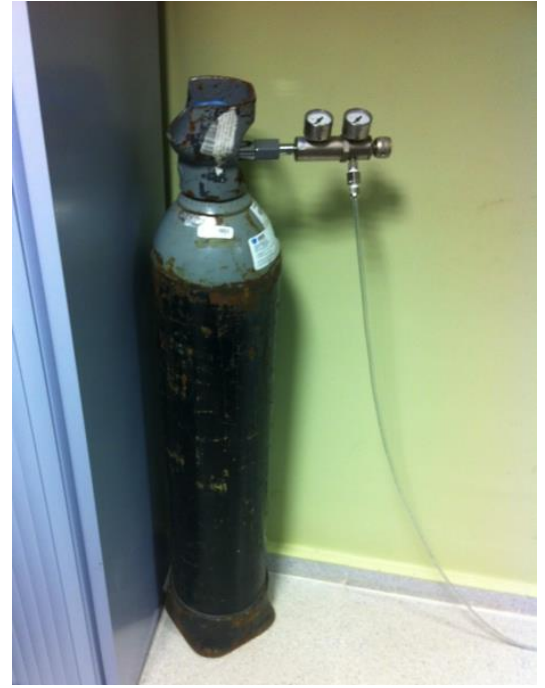

a

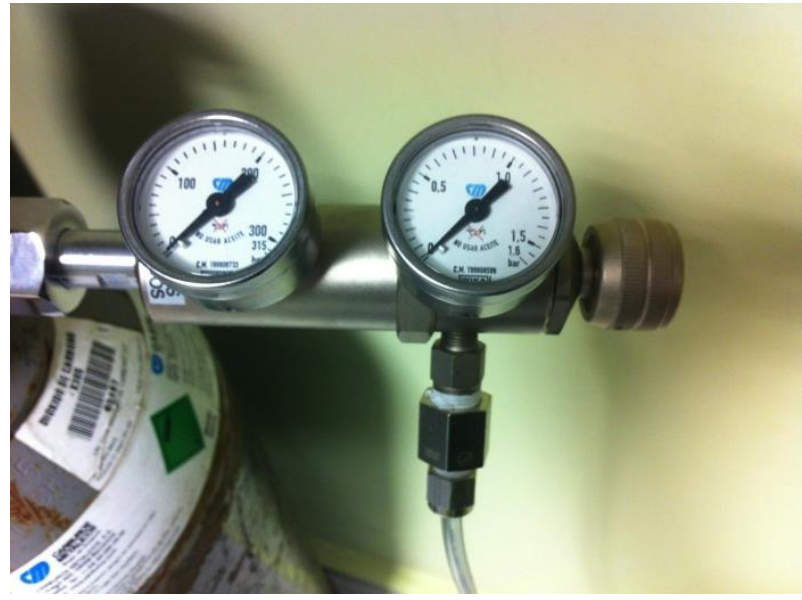

$\mathrm{b}$

Figure 3.8. - (a) $30 \mathrm{~L} \mathrm{CO}_{2}$ gas bottle and (b) detail of the pressure regulation valves and the rubber tube

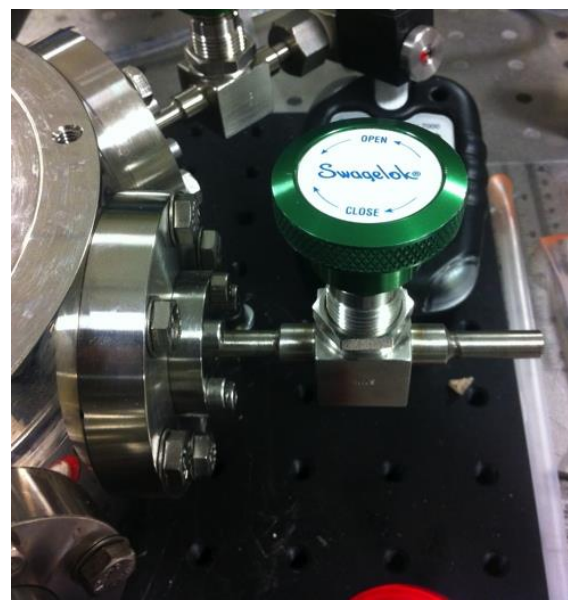

a

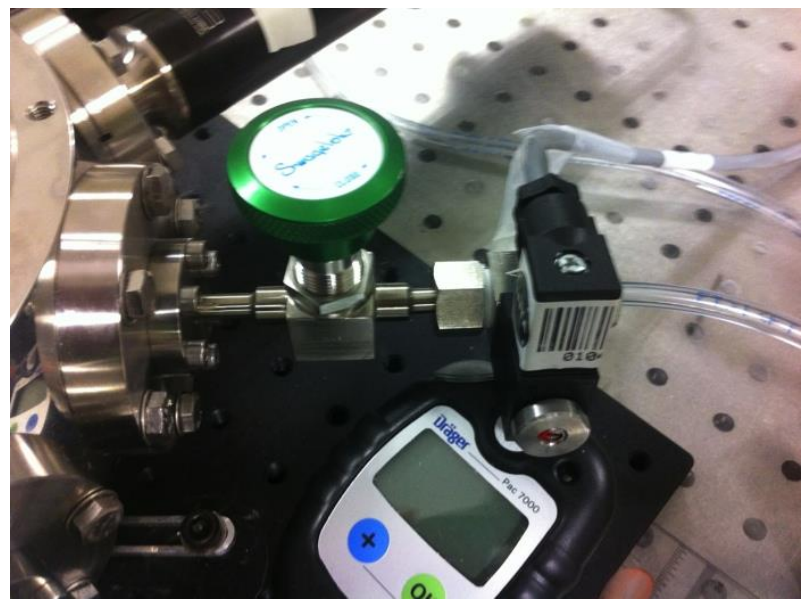

$\mathrm{b}$

Figure 3.9. - (a) Butterfly valve acting as an up-to-air valve and (b) butterfly valve in line with the gas flow valve and the gas line coming from the $\mathrm{CO}_{2}$ gas bottle

\subsubsection{Pressure controller}

The pressure sensor seen in section 3.2.1.3 and the electroactuated gas flow valve presented in section 3.2.1.6 are both connected to a RVC 300 pressure controller by Pfeiffer Vacuum (Figure 3.10). 


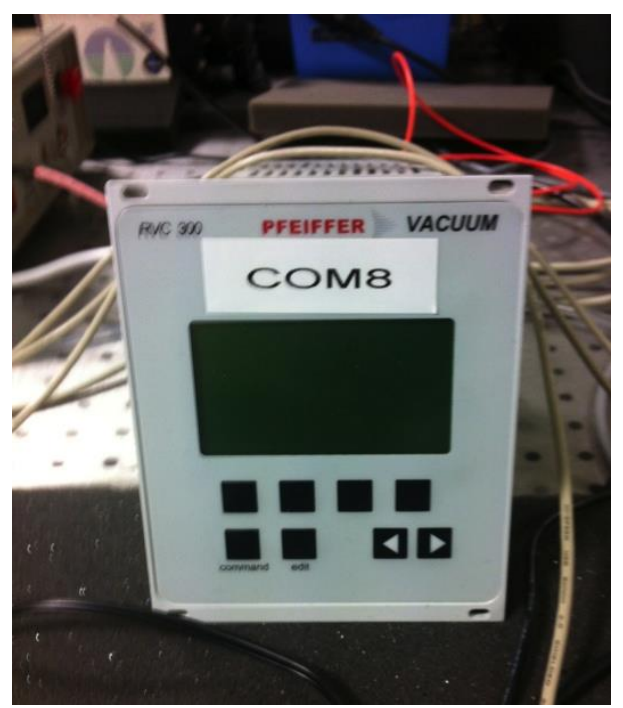

Figure 3.10. - RVC 300 pressure controller by Pfeiffer Vacuum

This pressure controller is computer commanded through RS232 serial port. The user establishes the desired pressure to be reached inside the $R L S$ ExoMars Simulation Chamber and this RVC 300, fed by the TPR280 pressure sensor, actuates on the gas flow valve to achieve this pressure condition. This is done thanks to a PID (Proportional, Integral and Derivative) controller. The pressure value oscillates around the desired pressure value or setpoint tending to it with each oscillation, as it will be seen in the test subsection.

At the same time and through the same communications port, the computer can interrogate the RVC 300 at any moment to keep track of the pressure inside the chamber.

\subsubsection{Refrigeration unit}

Apart from the pressure subsystem, it has also been mentioned that the sample has to be maintained at a certain temperature during the scientific operations. This is accomplished by pumping a cooled refrigeration liquid into the refillable container (RC) on top of which the sample is placed and prepared, and which will be presented in 3.3.2.1.

The refrigeration unit that cools and pumps this refrigeration liquid is a Ministat 230 compact cooling bath circulation thermostat by Huber Kältemachinenbau (Figure 3.11), capable of cooling a liquid down to $-40^{\circ} \mathrm{C}$, the lower limit for the operating temperature of the RLS instrument (Ball, 2013). The 
liquid being used for the experiments is methanol, which has a freezing point of $97.6^{\circ} \mathrm{C}$, well below the minimum cooling temperature of the unit.

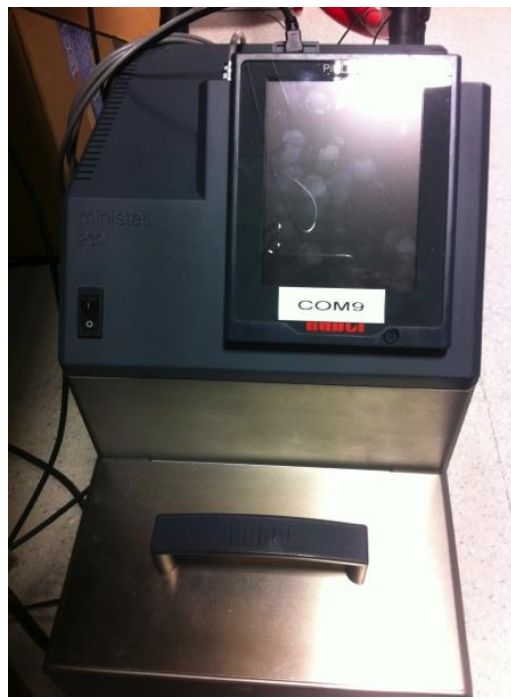

Figure 3.11. - Huber Ministat 230 with Pilot ONE controller.

This refrigeration liquid is pumped to the RC through two liquid lines mounted in a fluid feedthrough by MDC Vacuum (Figure 3.12). The liquid flows to and from this feedthrough with the help of two 1.5m-long isolated stainless steel hoses, capable of transporting liquids at temperatures as cool as $-50^{\circ} \mathrm{C}$.

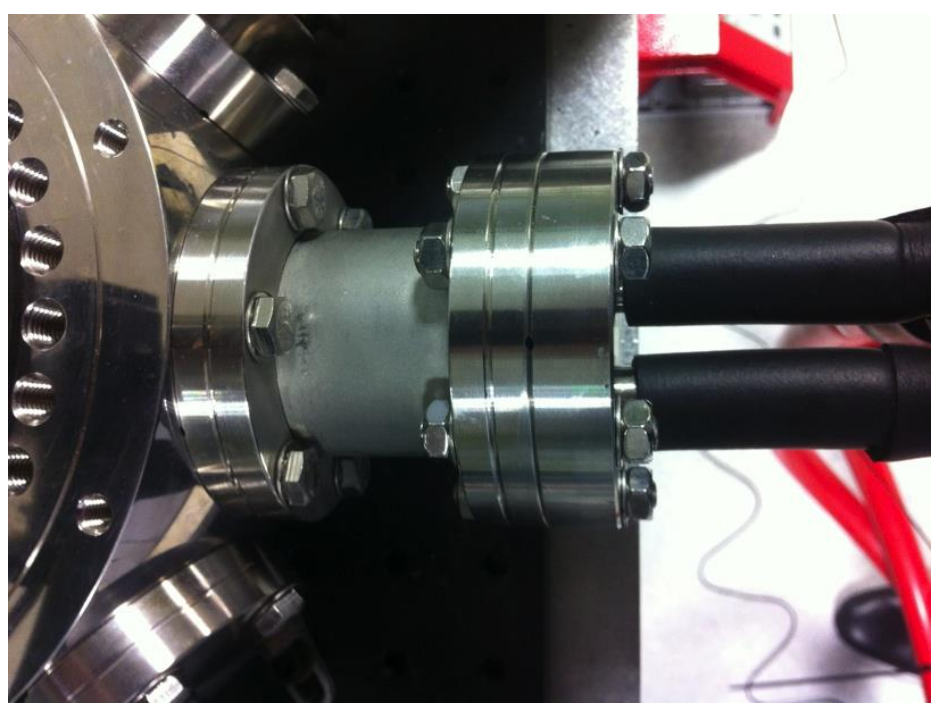

Figure 3.12. - Fluid feedthrough by MDC Vacuum with two lines isolated with neoprene. The red tubes on the lower right part of the image are the $1.5 \mathrm{~m}$ long isolated stainless steel hoses.

The Huber Ministat comes with a controller, the Pilot ONE, which allows for the commandment of functional parameters such as the pumping speed, the security temperature upper and lower limits, and of course the desired 
temperature inside the bath or at the element being refrigerated. For reading and controlling the temperature of the process, the Pilot ONE has a PT100 input port. Figure 3.13 shows the vacuum feedthrough for a $2 x 2$ pin LEMO connector built by IPT Albrecht, that leads to the PT100 installed inside the RC.

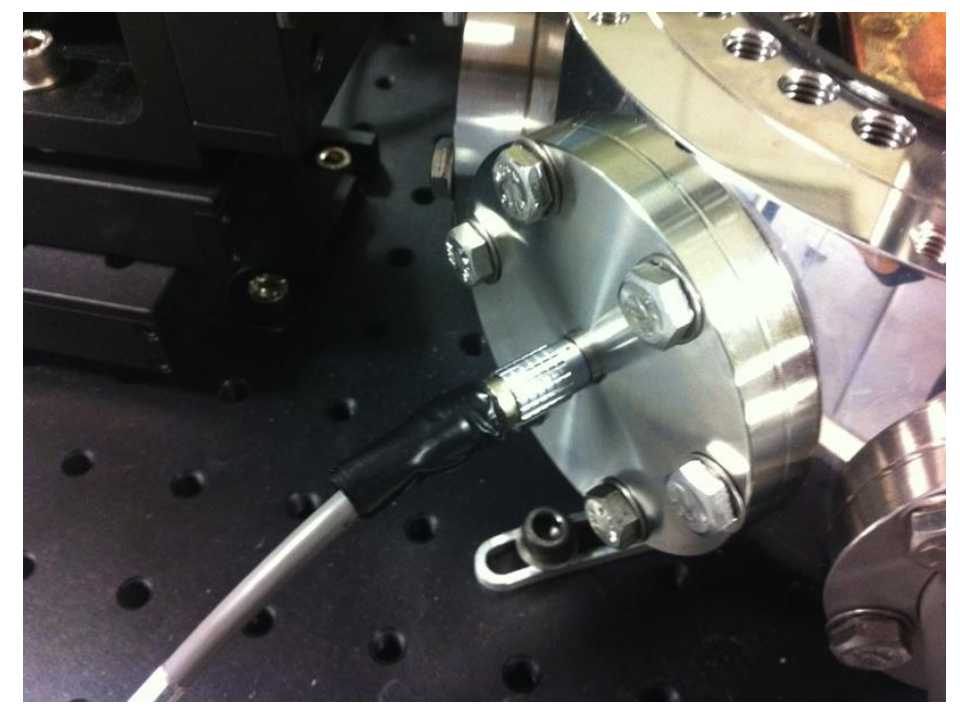

Figure 3.13. - LEMO feedthrough for the temperature measurement of the process on the RC through a PT100 sensor.

As with the RVC 300, the Pilot ONE can be commanded from a computer through a USB communications port. With it, the current temperature at the bath or at the process can be known at any time and the desired temperature established.

In the event of using this chamber for other purposes beyond the RLSExoMars simulation, and requiring lower refrigeration temperatures, the fluid feedthrough and the materials used for the construction of the RC are capable of withstanding cryogenic temperatures and nitrogen in liquid or gas state could be pumped substituting the current refrigeration unit and hoses.

\subsubsection{Pressure and temperature test}

Once having presented the building blocks of the RLS-ExoMars Simulation Chamber, it is time to show the pressure and temperature curves (Figure 3.14) obtained reading these values from the RVC 300 and the Pilot ONE, respectively, every second. The vertical scales have been reduced to appreciate the lower limit 
reached in temperature and the relaxed oscillations produced in the pressure readings.

The final temperature was set to $-16^{\circ} \mathrm{C}$ for this test for two reasons: (1) the time for reaching this temperature is lower than for lower temperatures and it is inside the limits defined in the scientific requirements for the analysis of samples by the RLS instrument (Ball, 2013), and (2) the Huber Ministat came with a malfunctioning problem that did not allow further lowering the temperature. This second problem is to be addressed by sending the unit back to the manufacturer.

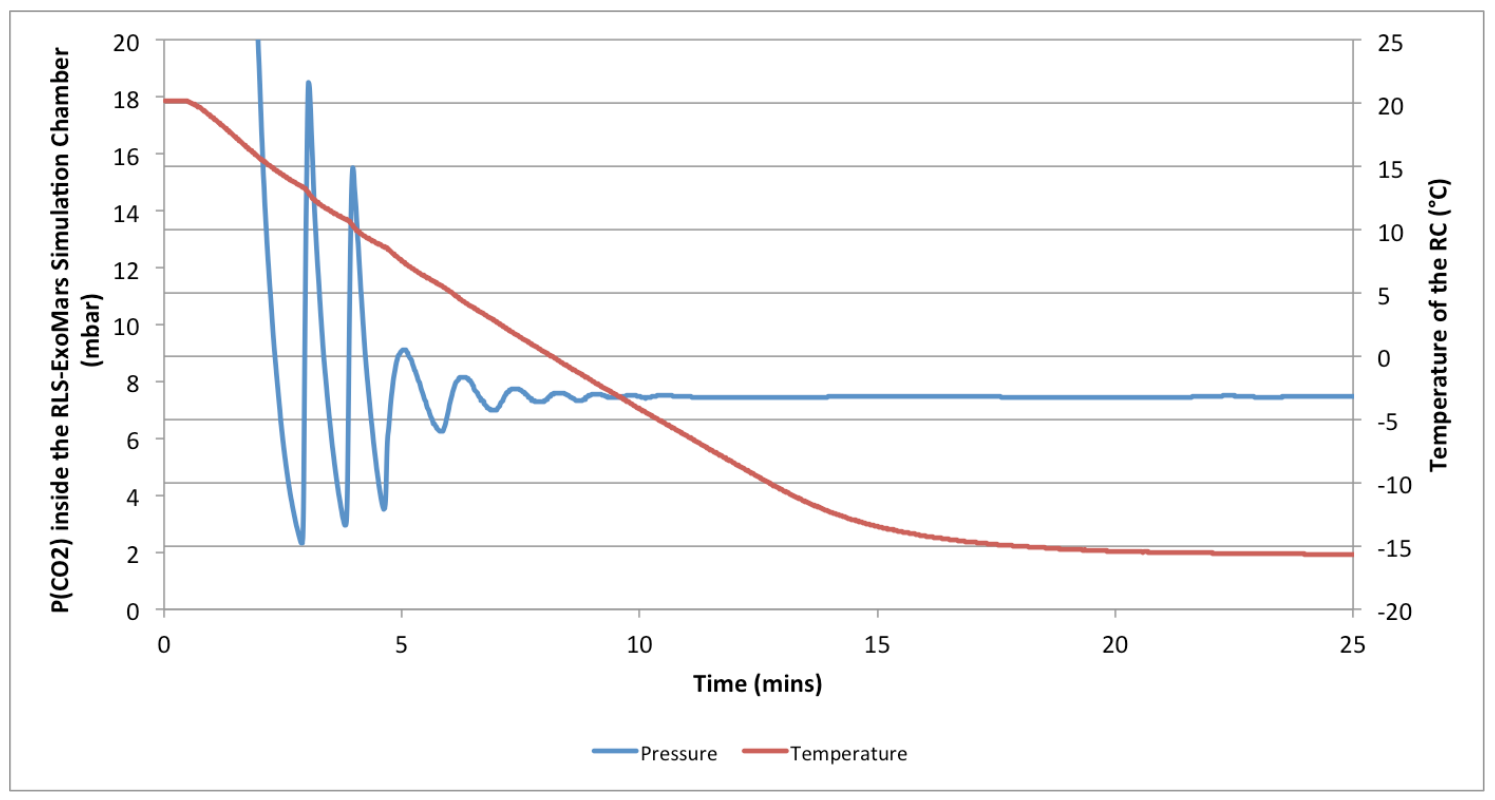

Figure 3.14. - Pressure and temperature curves without thermal load on the RC

Figure 3.14 shows in blue the $\mathrm{CO}_{2}$ effective pressure inside the $R L S$-ExoMars Simulation Chamber (values on the left vertical scale), and in red the temperature values at the RC (values on the right vertical scale).

Once the chamber is closed, the pressure is lowered from laboratory conditions to Mars conditions with the help of the vacuum generation station and the inflow of gas. The relaxed oscillations for the pressure values are due to the PID controller of the RVC 300. The amplitude of the initial oscillations depends on how much time before the pressure crosses the desired pressure setpoint passes since the gas bottle is opened and begins to accumulate inside the rubber tube. After these oscillations, the pressure progressively tends to the desired value, 
around 7.5mbar. Having reached the desired pressure, both the vacuum pump and the gas inflow continue running, assuring the atmosphere is kept stable until the end of the operations, when these units are stopped and the up-to-air valve is actuated to return to the laboratory atmospheric conditions.

Cooling and pumping liquid methanol, as explained before, allows reaching the final temperature. The temperature values tend slowly to the final value below $-15^{\circ} \mathrm{C}$. It can be noticed that the oscillations produced in pressure induce in the temperature values small variations.

The final combined environmental conditions are reached in less than 30 minutes, which is a reasonable time to wait for the beginning of the experiments.

From this test it can be thus concluded that both the temperature and pressure subsystems, and the computer communications that record the values work properly. Normal scientific operations can begin from this point. 


\subsection{Sample system}

The sample system refers to all those elements that allow for the proper analysis of the sample from which geochemical information is to be extracted.

In the RLS instrument, the sample is extracted from vertical surveys down to $2 \mathrm{~m}$ deep in the Martian subsurface. After the sample, a solid cylindrical core of $3 \mathrm{~cm}$-long and $1 \mathrm{~cm}$ in diameter, is extracted, it is incorporated to the ExoMars rover through the Core Sample Transportation Mechanism (CSTM). This operation is performed the next sol after extraction, to ensure there is enough power to proceed with the sample preparation steps. A sol is a Martian day, which lasts a little bit longer than that on Earth, around 24h $37 \mathrm{~m}$.

Once the sample is inside the rover, it is delivered to the Crushing Station (CS), part of the Sample Preparation and Distribution System (SPDS). The CS crushes the sample by repeatedly compressing it between two jaws. The powder generated from this crushing process, once it has a size below $500 \mu \mathrm{m}$, is passed to the Dosing Station (DS). After the crushing process is completed, the grain size distribution of the resulting powder is confined in the range of $10 \mu \mathrm{m}$ to $500 \mu \mathrm{m}$, with an average grain size below $250 \mu \mathrm{m}$, approaching to the scientific requirements (Ball, 2013).

The DS, being loaded with this powder sample, is the responsible of vibrating and dosing it on the Refillable Container (RC). The RC will host most of the sample during the analyses to be performed on an experiment cycle. Most of the sample because, prior to the analyses, the sample has to be prepared. This preparation consists of a flattening of the powder, so to obtain a smooth surface with grain irregularities of no more than $200 \mu \mathrm{m}$ on the $80 \%$ of the surface of the RC with no less than $50 \%$ of the grains under $100 \mu \mathrm{m}$ from the surface of the RC. This flattening process is performed through the passing of the RC under a Powder Sample Flattening Mechanism (PSFM) or blade, which provides this smooth surface and discards the excess of powder. The objective of this flattening from the point of view of the RLS instrument is to have the powder sample within the operating range of the autofocus mechanism incorporated to the Raman Optical Head (+/- 1mm (TBC)). 
The sample is then ready for analysis, placing the $\mathrm{RC}$, which contains it below the several analytical instruments in the Analytical Laboratory Drawer (ALD) by means of the Sample Preparation and Distribution System (SPDS) to which the RC is attached.

The RC and PSFM are reproduced for the RLS-ExoMars Simulation Chamber in order to have the sample prepared under the RLS environmental conditions $(\mathrm{P}=$ Martian, $\left.\mathrm{T}<-5^{\circ} \mathrm{C}\right)$. They are presented in the next section along with the optical window that allows the visual and spectroscopic examination of the sample.

\subsubsection{Parts}

\subsubsection{Refillable Container (RC)}

Figure 3.15 shows the real RC built by Kayser-Threde GmbH (KT) at Munich, which is integrated in the Powder Sample Handling System (PSHS). As the carousel in which it rests is curved, so is the RC. This allows the central line of analysis to coincide with the central line of the RC.

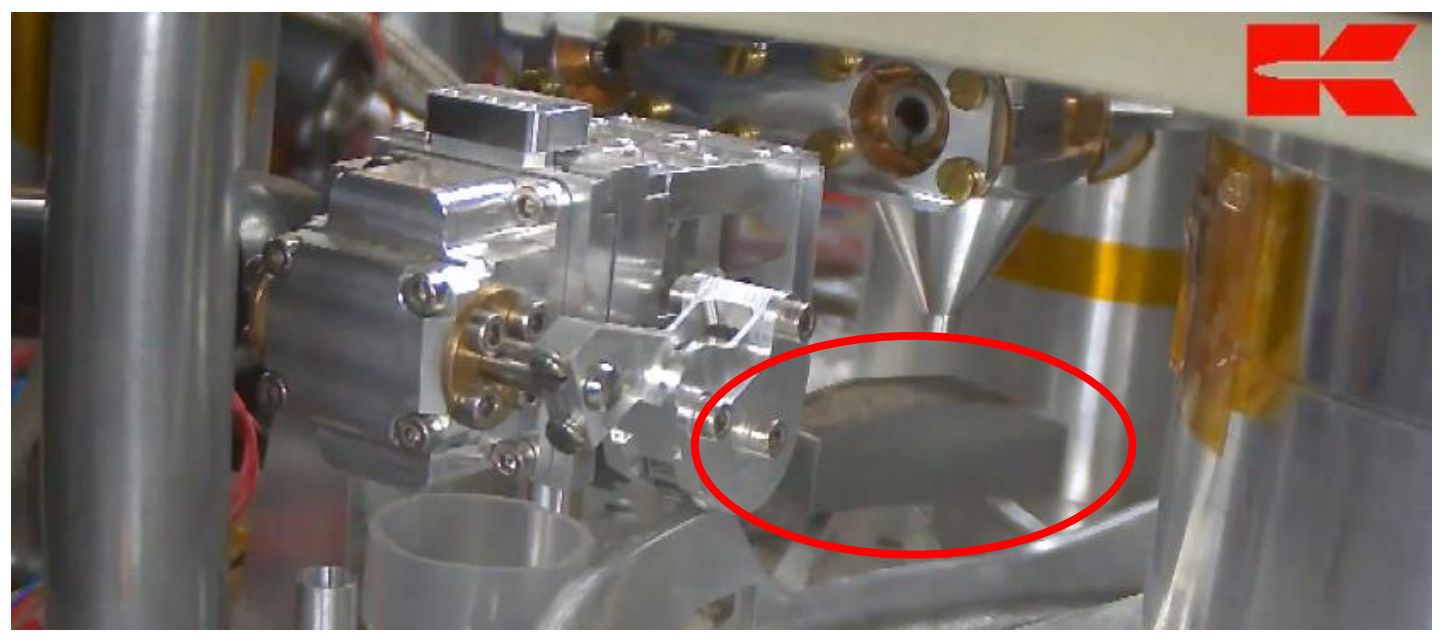

Figure 3.15. - RC built by KT (Credit: Kayser-Threde GmbH.)

For the RLS-ExoMars Simulation Chamber, this RC has been designed and built straight although it shares the profile of the original design. The reason for this straightness is the same as for the PSHS: the movement of the Raman optical head during the analysis is straight along the major length of the RC. Figure 3.16 shows a picture of the whole chamber with the cover removed, unveiling its content. 


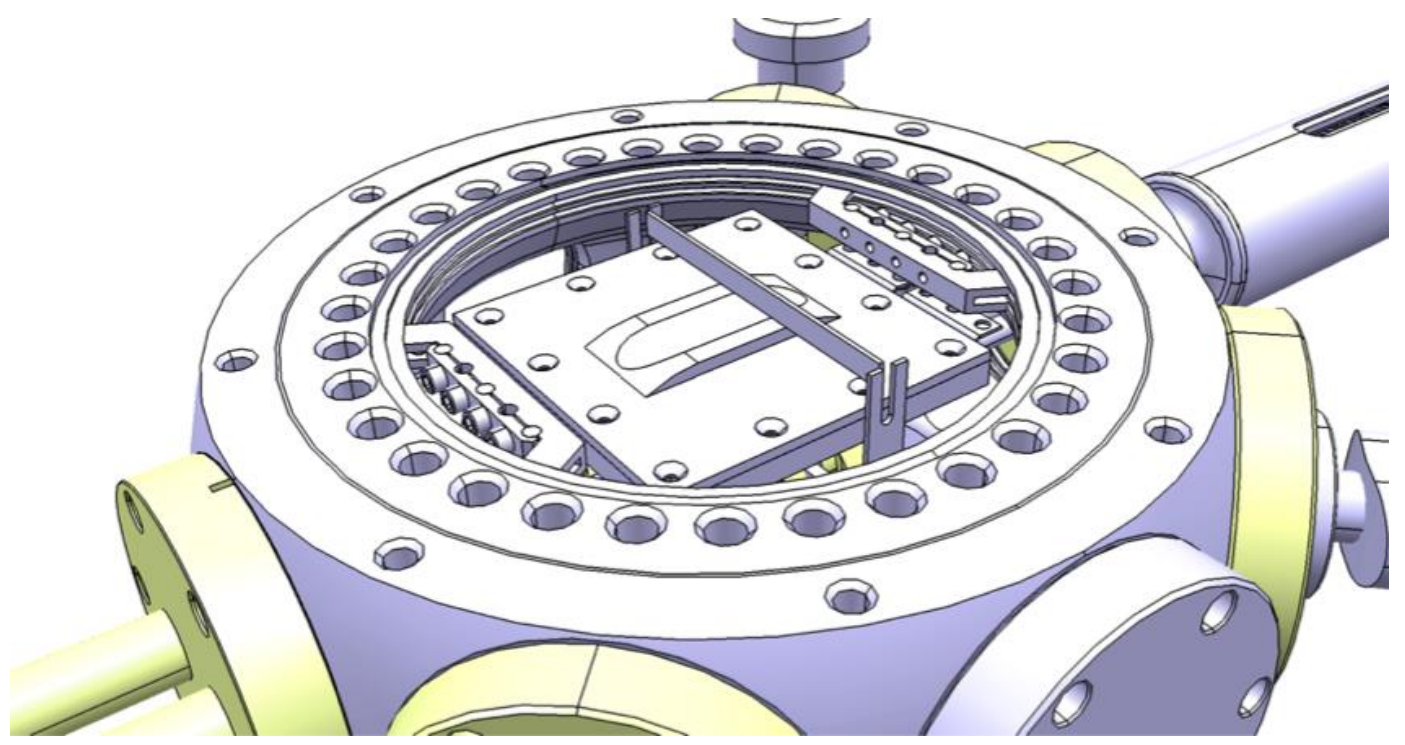

Figure 3.16. - RLS-ExoMars Simulation Chamber: detail of the interior.

The interior of the chamber shows a square with the RC machined in its center. This is the shape the RC takes for this simulation chamber. The central part where the sample is deposited has a groove with a length of $\sim 21 \mathrm{~mm}$ and a width of $\sim 11 \mathrm{~mm}$. The volume of sample that it can hold approaches $3 \mathrm{~cm}^{3}$. The square profile completes two purposes: (1) hosting an inner small deposit for the refrigeration liquid to flow and cool the RC, and (2) have a large area where the excess of sample can go during the flattening process, instead of falling into the chamber where it is more difficult to be removed during the cleaning process and has the risk of being absorbed by the pumping station with the associated hazard for the blades of the turbomolecular pump, turning at more than $20000 \mathrm{rpm}$.

With respect to the purpose number (1), Figure 3.17 presents an exploded view of the RC inside the RLS-ExoMars Simulation Chamber, where this small deposit ( ${ }^{4} 4.6 \mathrm{~cm}^{3}$ in volume) can be seen. The two $1 / 4$ " tubes coming from the liquid feedthrough end up in it. 


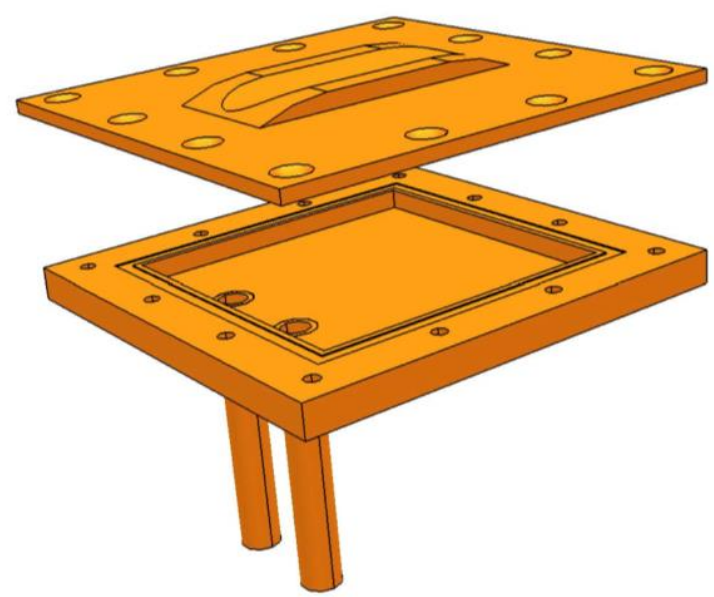

Figure 3.17. - Exploded view of the RC inside the RLS-ExoMars Simulation Chamber

Figure 3.18 shows the front view of the actual RC installed inside the chamber. The perimeter holes that can be seen in Figure 3.16 have been sealed along with the edges where the two pieces of the square meet to avoid the escape of refrigeration liquid to the exterior of the RC due to the suction effect that the low pressure inside the chamber applies on the deposit.

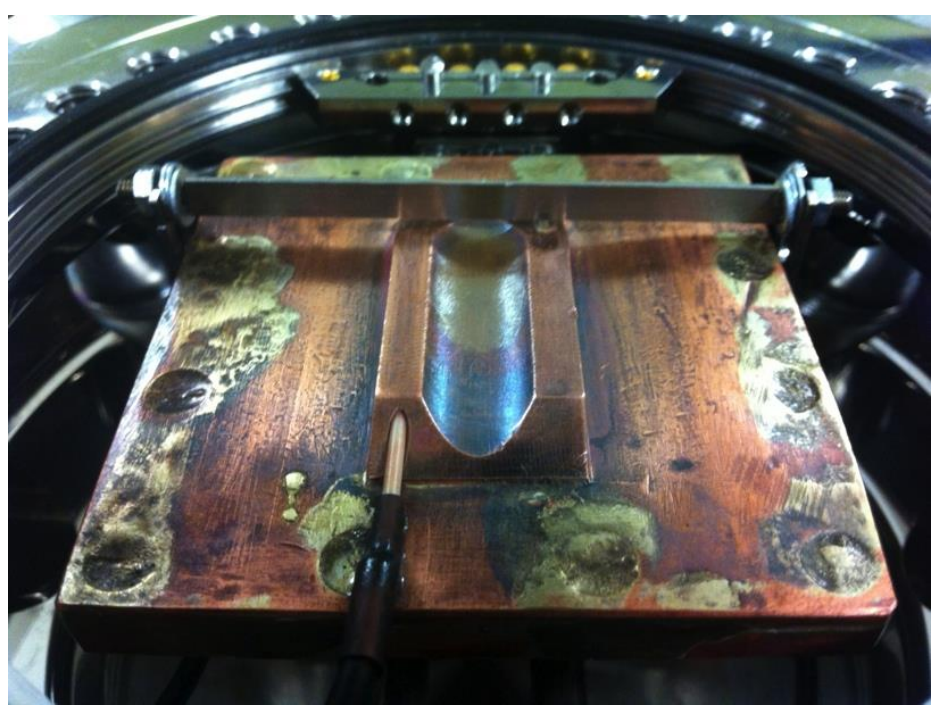

Figure 3.18. - Front view of the RC inside the RLS-ExoMars Simulation Chamber.

The RC has been machined in copper, a metal with very good thermal conductivity $\left(401 \mathrm{~W} \cdot \mathrm{m}^{-1} \cdot \mathrm{K}^{-1}\right)$, to maximize the thermal transmission between the sample and the refrigeration liquid. 
Another important characteristic that can be noticed from Figure 3.18 is the small cylindrical white thing inserted into the RC. This is the $15 \times 1.5 \mathrm{~mm} 1000 \mathrm{hm}$ class A PT100 sensor from RS Components that feeds the Pilot ONE controller at the Huber Ministat and helps monitoring and adjusting the temperature in real time. This PT100 is connected to the vacuum side of the LEMO feedthrough through the black cable that follows it.

The long and narrow metallic element placed on top of the RC is the blade that forms part of the flattening subsystem.

\subsubsection{Flattening subsystem}

The flattening subsystem is comprised of a motorized linear manipulator and a blade attached to its advancing rod at the vacuum side. Figure 3.19 shows the 3D layout of this subsystem with the RC as the target of the flattening process.

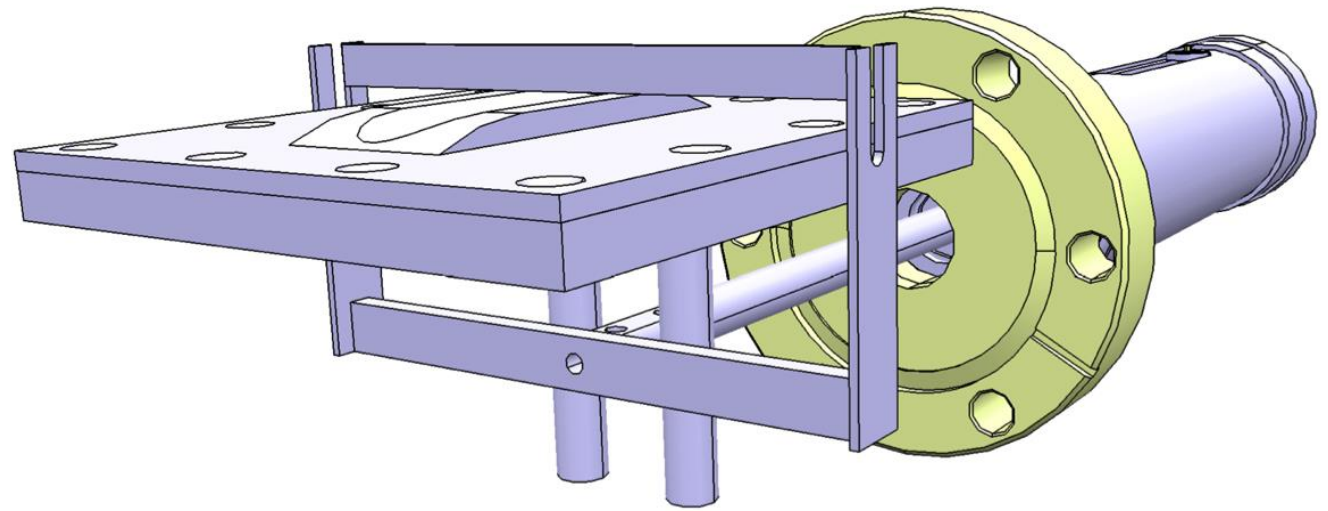

Figure 3.19. - 3D layout of the linear manipulator and the blade attached to it.

The vacuum part of the flattening subsystem, the elements on the foreground in Figure 3.19, is comprised of three elements: (1) the metallic rod that goes forward or backward as the actuator of linear manipulator is mechanically turned right or left, respectively; (2) a U-shaped structure screwed to the rod; and (3) a long, narrow and thin stainless steel piece that is held by the U structure and rests on top of the RC. This last element is referred to as the 'blade' and is the responsible of flattening the powder on the $\mathrm{RC}$ as the rod of the linear manipulator moves parallel to the large dimension of the RC. 
Figure 3.18 shows this blade resting at the 'home position' of the flattening process. As it moves, it will advance to the front part where the PT100 is inserted. Figure 3.20 presents a top view of the air section of the mechanically actuated linear manipulator by MDC Vacuum.

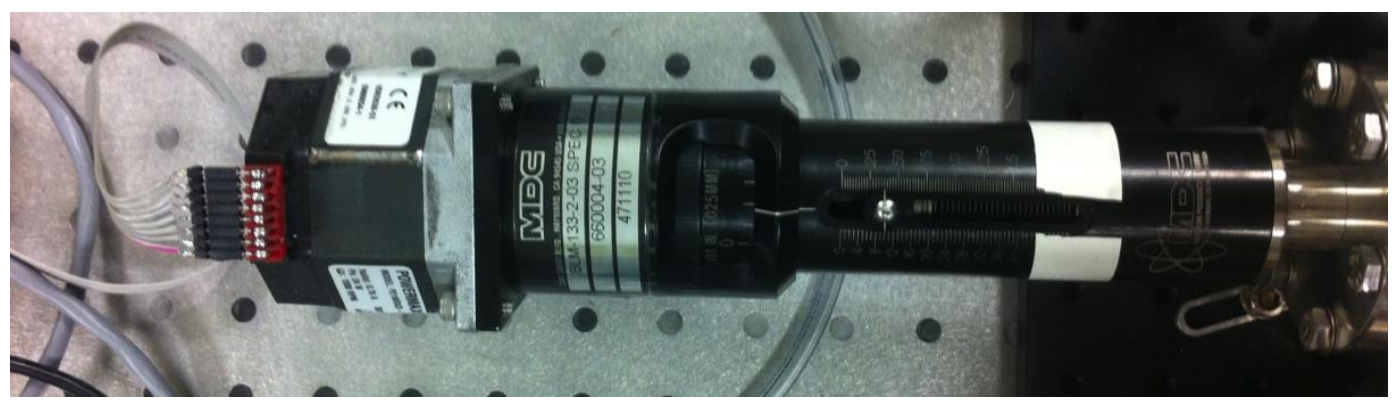

Figure 3.20. - Mechanically actuated linear manipulator by MDC vacuum, attached to the RLS-ExoMars Simulation Chamber.

On the left of the image, just before the logo of the manufacturer, there is a stepper motor. This motor, conveniently fed through the electrical wires soldered to the 8 motor pins in the red piece, turns right or left at a speed proportional to the shape of the pulsed signal passed through the wires.

This motor actuates on the clutch piece with the graduations on it, visible through the rectangular hole in the middle of the image. This piece, in turn, rotates and makes a screw go in or out the bellow that retains the vacuum inside the chamber and host inside the metallic rod previously presented. The barrel with the measuring scales on it marks how much the rod, and thus the blade, advances or retracts parallel to the $\mathrm{RC}$.

Figure 3.21 shows the circuit board with the electronic components that allow for the safe and smooth movement of the motor that makes the flattening process a reality. 


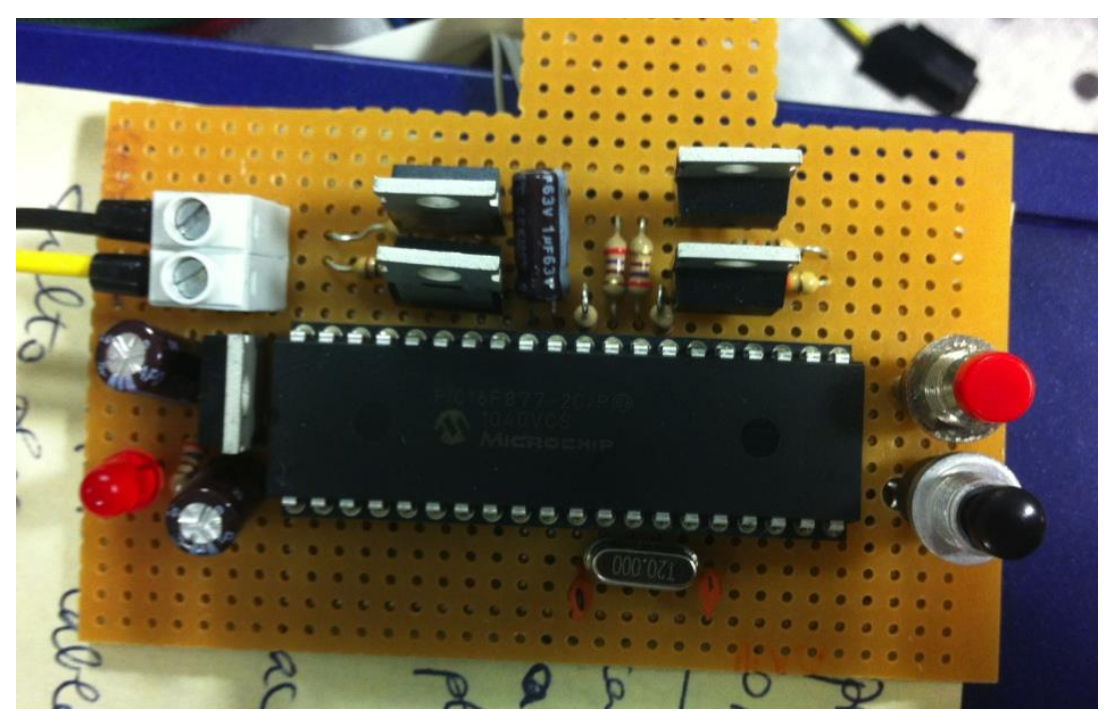

Figure 3.21. - Stepper motor controller for the linear manipulator of the flattening subsystem.

The details of this circuit board and the software that controls the stepper motor as the user actuates on the red and black buttons on the right, are depicted in Annex B.

\subsubsection{Optical Window}

Once the chamber has reached the desired environmental conditions and the sample on the RC has been flattened, the Raman spectrometer can proceed with the scientific analysis. In this simulation chamber as well as in the real RLS instrument, an optical window separates the interior and exterior of the chamber, preserving the pressure difference between both spaces.

Figure 3.22 shows the exploded view of the top cover of the RLS-ExoMars Simulation Chamber with a pocket for hosting a microscope slide, which acts as a cheap and easily replaceable but optically convenient window. The scientific specifications for this optical window establishes that its thickness is less than $2 \mathrm{~mm}$ to avoid having spectroscopic contribution of the glass in the Raman spectra of the sample being analysed. The sandwich structure shown in this 3D layout is comprised of: (1) a machined DN100CF vacuum thin flange a frame, (2) two Viton frames that embrace and seal the optical window between them, and (3) a metallic frame that crowns the layered structure and closes and compresses the assembly with a collection of screws that surrounds the window. 


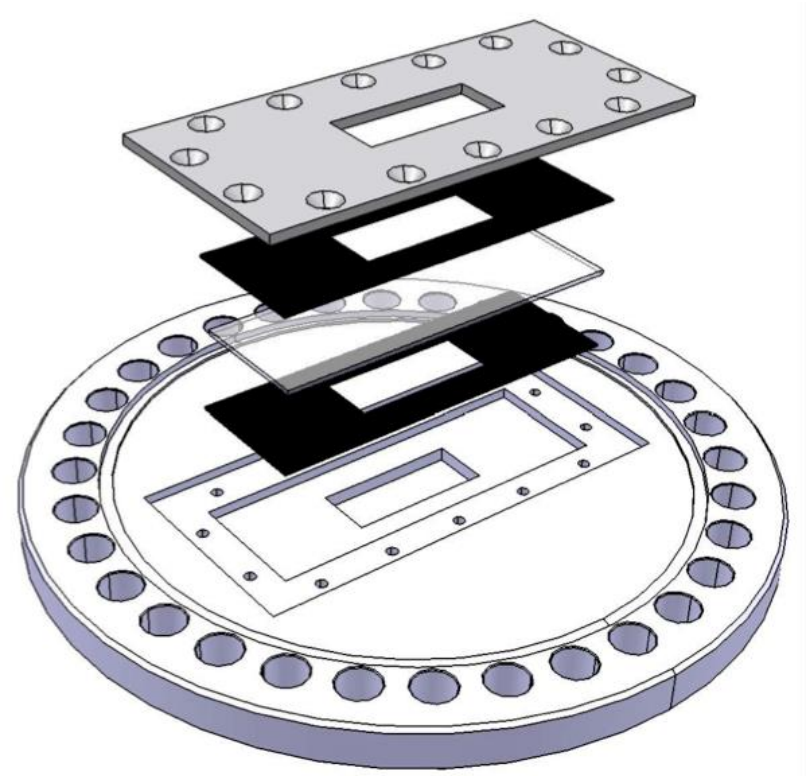

Figure 3.22. - Exploded view of the top cover of the RLS-ExoMars Simulation Chamber hosting a thin optical window.

The transmission curve of the optical window is presented in Figure 3.23. The green zone on the graph corresponds to the spectral region defined for the RLS instrument. Its response is quite uniform along the whole range of wavelengths.

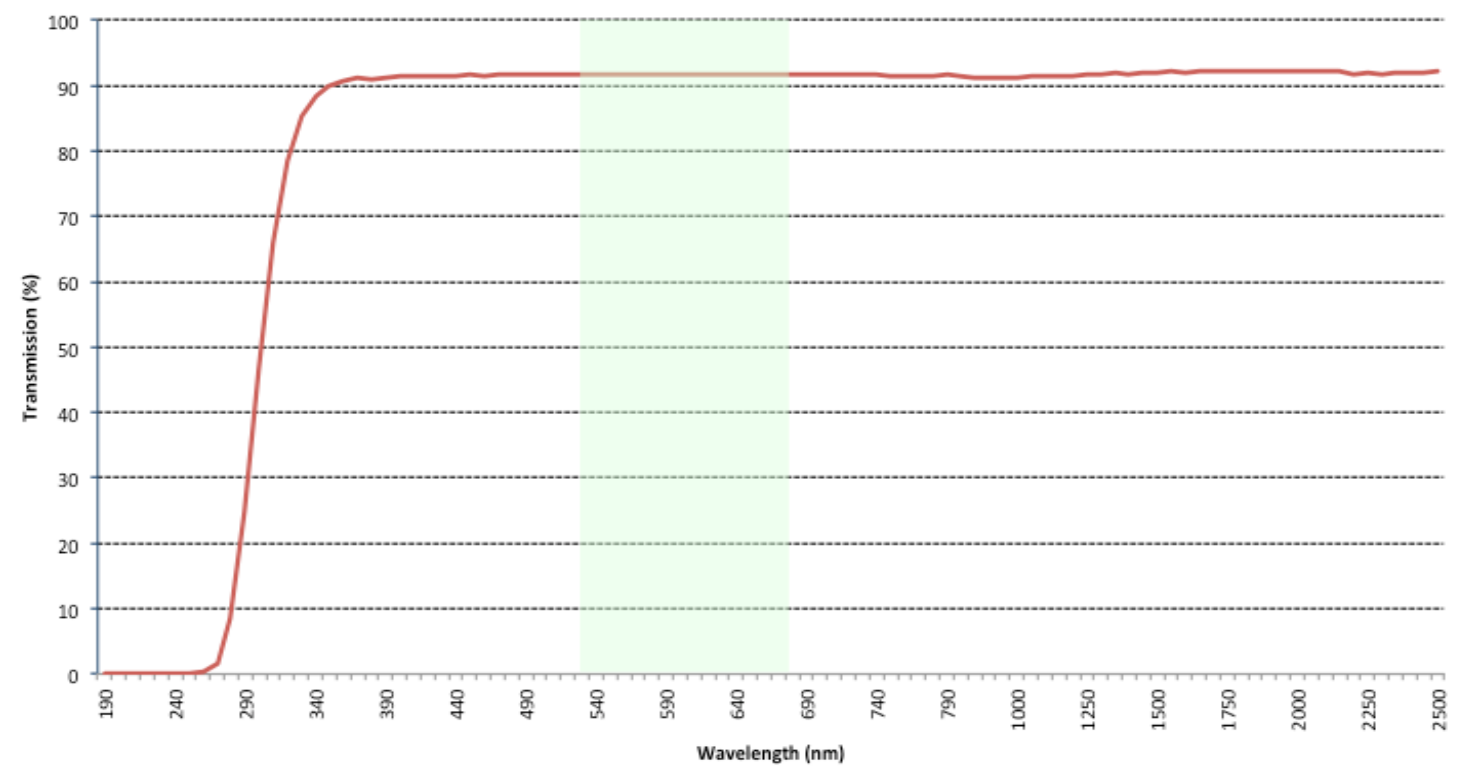

Figure 3.23. - Transmission curve of the optical window used for the $R L S$ ExoMars Simulation Chamber.

Figure 3.24 is a picture of the actual top cover of the RLS-ExoMars

Simulation Chamber, with the optical window assembly mounted and working. 
Despite its thickness $(1.00+/-0.05 \mathrm{~mm})$, it has not yet been replaced due to break. With the objective of increasing the sealing of the layered structure, vacuum grease was extended on both sides of each Viton frame.

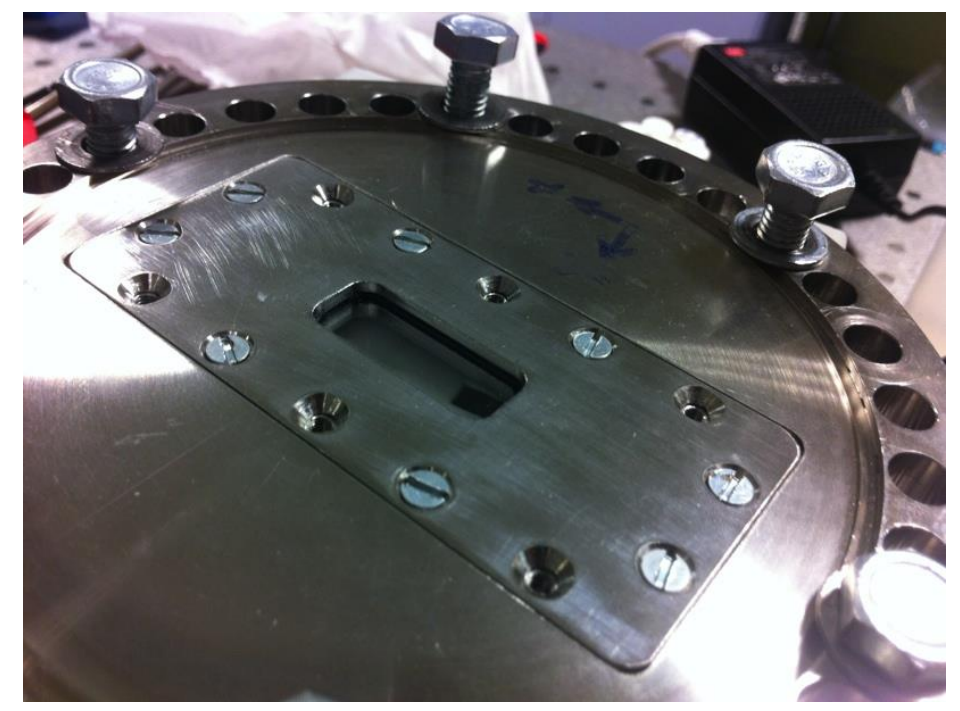

Figure 3.24. - Detail view of the optical window assembly integrated with the top cover of the RLS-ExoMars Simulation Chamber.

\subsubsection{Sample system test}

The aim of this test is to show how good is the flattening system in getting a smooth surface on the RC for both dry and hydrated samples.

The current protocol in the real Powder Sample Dosing and Distribution System (PSDDS) contemplates the deposition of four sample dosings of $0.1 \mathrm{ml}$ along the RC before the first flattening operation and, after that, two more dosings at the beginning of the RC intercalated with their correspondent flattenings. These two extra operations are focused in filling the possible holes left on the surface of the sample during the first flattening due to, for example, trenches created by dragging the largest crystals.

In the RLS-ExoMars Simulation Chamber this flattening is performed only once after two or three sample dosings along the RC. The reason is that this operation is executed under the RLS-ExoMars relevant conditions with the chamber closed and with no possibility of further dosings.

Although in Chapter 5 two samples are used to run analysis on thermolability, the present test has been done for other two samples. These 
samples have been selected to have different hydration, which might lead to grains clumping and thus differences in terms of flattening performance.

For each material, the heights of the mineral grains in a matrix of three $500 \mu \mathrm{m}$-spaced lines across the RC by twenty $500 \mu \mathrm{m}$-spaced points along it, are measured. This height is given with respect to the top surface of the RC.

\section{Quartz $\left(\mathrm{SiO}_{2}\right)$}

This is a dry sample where the mineral grains are visually non sticky, which is supposedly advantageous for the flattening process. The grain size distribution of the sample was selected so that it resembled the one obtained by KT in their tests with fused silica (FS120) as seen in (Tim, 2012). This distribution is an asymmetric Gaussian with its mean around $250 \mu \mathrm{m}$, upper limit in $500 \mu \mathrm{m}$ and lower limit around $10 \mu \mathrm{m}$.

Figure 3.25 shows pictures of the quartz sample before and after the flattening process. The image before was taken after dosing onto the RC and the image after was taken once the chamber was re-opened at the end of the experiment.

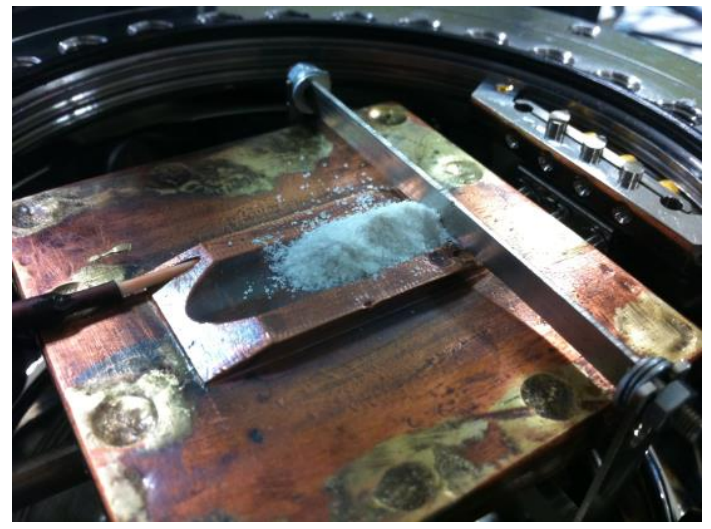

a

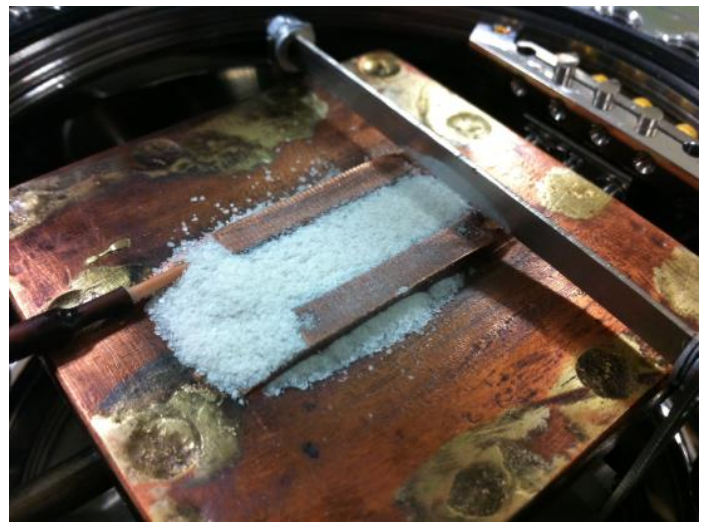

$\mathrm{b}$

Figure 3.25. - Image of quartz sample before (a) and after (b) the flattening process

A visual examination shows that the surface does not end up being very smooth, presenting some trenches and holes. This could be due to the dragging of

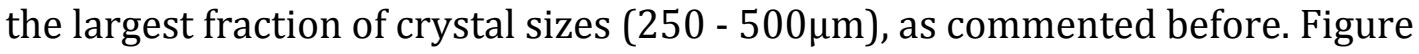


3.26 translates Figure 3.25.b into distances, from the surface of the RC to the mineral grain, and for three central lines along the RC.

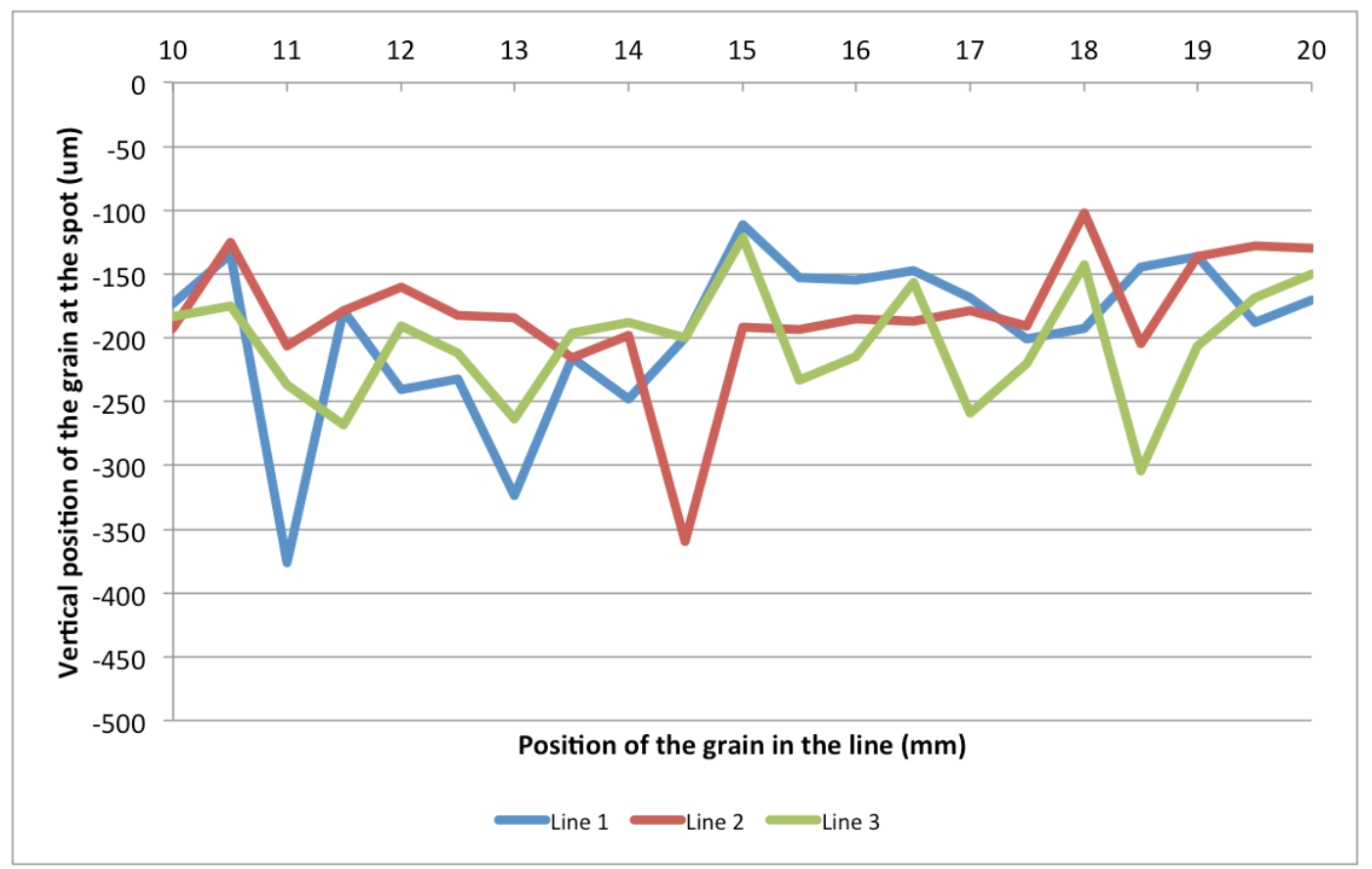

Figure 3.26. - Focused positions for the quartz mineral grains of three lines along $10 \mathrm{~mm}$ of the RC. Line 1 is the central line $+500 \mu \mathrm{m}$; Line 2 is the central line; and Line 3 is the central line $-500 \mu \mathrm{m}$.

Table 3.1 presents the statistics for these three lines.

\begin{tabular}{|c|c|c|c|}
\hline & Line 1 & Line 2 & Line 3 \\
\hline \multirow{2}{*}{$<100 \mu \mathrm{m}$} & $0 \%$ & $0 \%$ & $0 \%$ \\
\hline $200 \mu \mathrm{m}$ & $67 \%$ & $81 \%$ & $52 \%$ \\
\hline $\begin{array}{c}\text { Average height }(\mu \mathrm{m}) \\
\text { Standard deviation }(\mu \mathrm{m})\end{array}$ & -195 & -182 & -204 \\
\cline { 2 - 4 } & 64 & 51 & 46 \\
\hline
\end{tabular}

Table 3.1. - Statistics for the three lines of points on the quartz sample after the flattening process.

As it can be observed in it, the flattening process does not accomplish the scientific requirement imposed to the PSFM, as presented in Section 3.3. However, understanding the limitations of this procedure compared to the protocol performed with the real PSDDS, and having a standard deviation from the average height of less than $100 \mu \mathrm{m}$, this result can be accepted as ready for Raman. 


\section{Gypsum $\left(\mathrm{CaSO}_{4} \cdot 2 \mathrm{H}_{2} \mathrm{O}\right)$}

The second selected sample is a hydrated sample. Theoretically, due to the stickiness of hydrated samples, which increases with the level of hydration, the flattening process will be harder and return poorer results.

As with the quartz, the grain size distribution follows the results obtained by KT in their PSDDS tests for a quartz sample (Tim, 2012). This looks again like an asymmetric Gaussian distribution with limits in $10 \mu \mathrm{m}$ and $500 \mu \mathrm{m}$ and with the average of the distribution in a value around $250 \mu \mathrm{m}$.

Figure 3.27 shows the images before and after flattening the gypsum powder material. It can be noticed that the appearance of the sample is of less loose grains, looking like flour, what translates into a poor flattened surface.

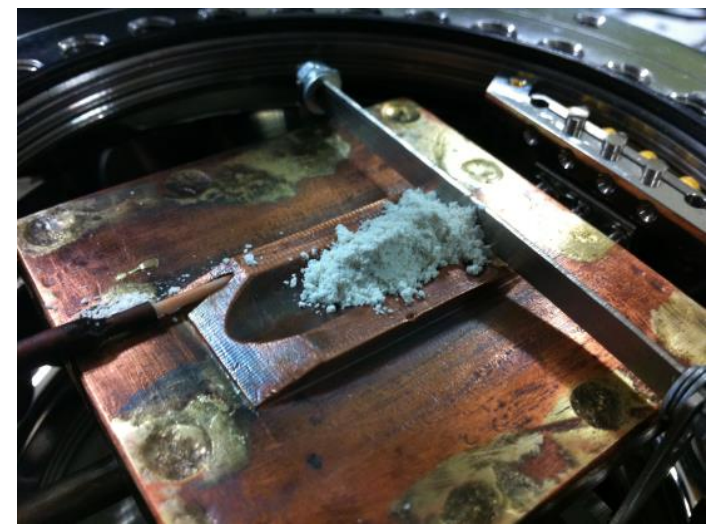

a

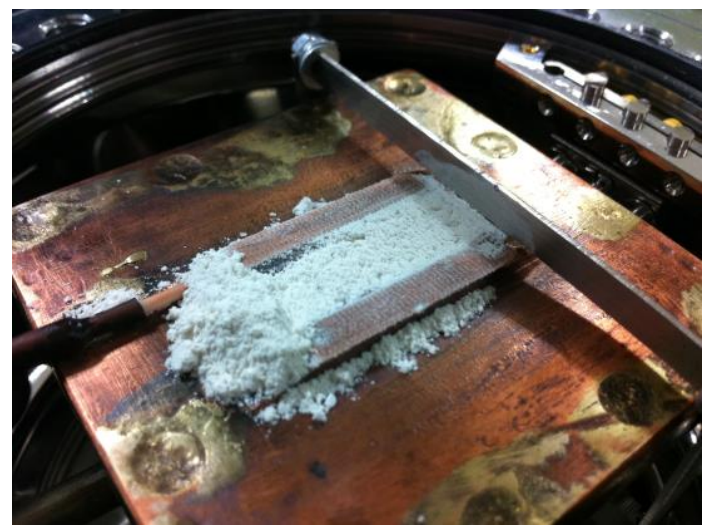

b

\section{Figure 3.27. - Image of gypsum sample before (a) and after (b) the flattening process}

Figure 3.28 zooms into the three central lines along the RC of the Figure 3.27.b, showing the high variability that is visually noticed in the image. The behaviour of the three lines along the RC is similar, showing a pattern in how the mineral grains of this hydrated sample are dragged by the flattening tool. 


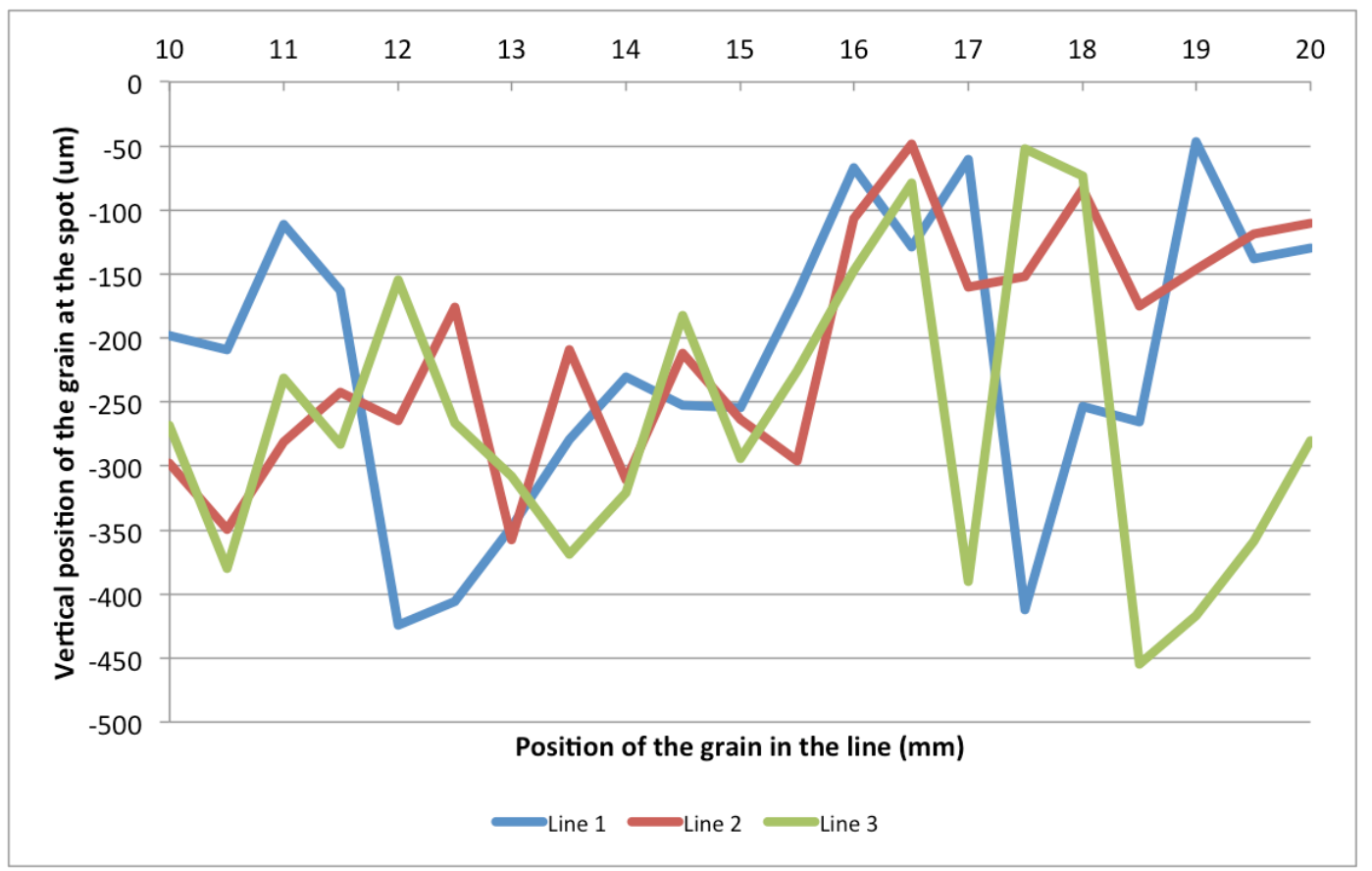

Figure 3.28. - Focused positions for the gypsum mineral grains of three lines along $10 \mathrm{~mm}$ of the RC. Line 1 is the central line $+500 \mu \mathrm{m}$; Line 2 is the central line; and Line 3 is the central line $-500 \mu \mathrm{m}$.

The translation of Figure 3.27 into data is reflected in Table 3.2.

\begin{tabular}{|c|c|c|c|}
\hline & Line 1 & Line 2 & Line 3 \\
\hline$<100 \mu \mathrm{m}$ & $14 \%$ & $10 \%$ & $14 \%$ \\
\hline$<200 \mu \mathrm{m}$ & $48 \%$ & $48 \%$ & $29 \%$ \\
\hline Average height $(\mu \mathrm{m})$ & -216 & -208 & -263 \\
\hline Standard deviation $(\mu \mathrm{m})$ & 114 & 90 & 115 \\
\hline
\end{tabular}

Table 3.2. - Statistics for the three lines of points on the gypsum sample after the flattening process.

Once again, and worse this time, the vertical position of the mineral grains does not reach the desired values although the average height remains similar for the three lines, and so does the standard deviation, a little bit higher in this case.

These tests reinforce the idea of performing several dosings and flattenings to the real sample deposited onto the $\mathrm{RC}$, with the aim of accomplishing the scientific requirements for the PSDDS in the context of the mission. The vibration of the sample inside the DS before each dosing is a must in order to loose the mineral grains that, due to the chemistry of the sample, could be susceptible of sticking together and thus hindering the flattening procedure. 


\section{Chapter 4}

\section{RLS-ExoMars Science}

\section{Simulator}

The Powder Sample Handling System (PSHS) moves the Refillable Container (RC) below the scientific instruments that will analyse the sample on it.

In the specific case of the RLS instrument, the RC will present a number greater or equal to 20 points angularly distributed along the sample to the Raman Optical Head at the other side of the optical window, outside the Ultra Clean Zone (UCZ) of the Analytical Laboratory Drawer (ALD).

Translated to the RLS-ExoMars Simulation Chamber, in which the RC remains static, is the Raman Optical Head $(\mathrm{OH})$ the one that has to be moved above and along the sample. For doing this, an optomechanic system has been designed and built to work in conjunction with the chamber. This has been called RLS-ExoMars Science Simulator. It is based on the Science Simulator designed to simulate the operation mode of the RLS instrument (López-Reyes et al., 2013), with some modifications to make it more compact and precise. 



\subsection{Introduction}

The RLS-ExoMars Science Simulator is an optomechanical system comprised of a XYZ positioning system and a Raman Probe + optical assembly, which is positioned over the sample on the RC.

The equivalent to the optical part of this Science Simulator in the case of the Raman Laser Spectrometer (RLS) is the Internal Optical Head (IOH).

This is the element that concentrates onto the sample the light coming from the laser through the excitation path. It also collects the Raman light generated on that spot and transports it to the spectrometer through the collection path. As the laser is operated in continuous wave, both the excitation and collection paths are used simultaneously. For avoiding the imaging sensor of the spectrometer from collecting the intense light of the laser, which would saturate it affecting the acquisition of a Raman spectrum, the IOH incorporates in its collection path an edge filter. This edge filter cuts or aggressively attenuates all the wavelengths below $150 \mathrm{~cm}^{-1}$ from the laser excitation wavelength. Figure 4.1 shows a drawing of the IOH as designed by Kayser Threde GmbH (KT). 


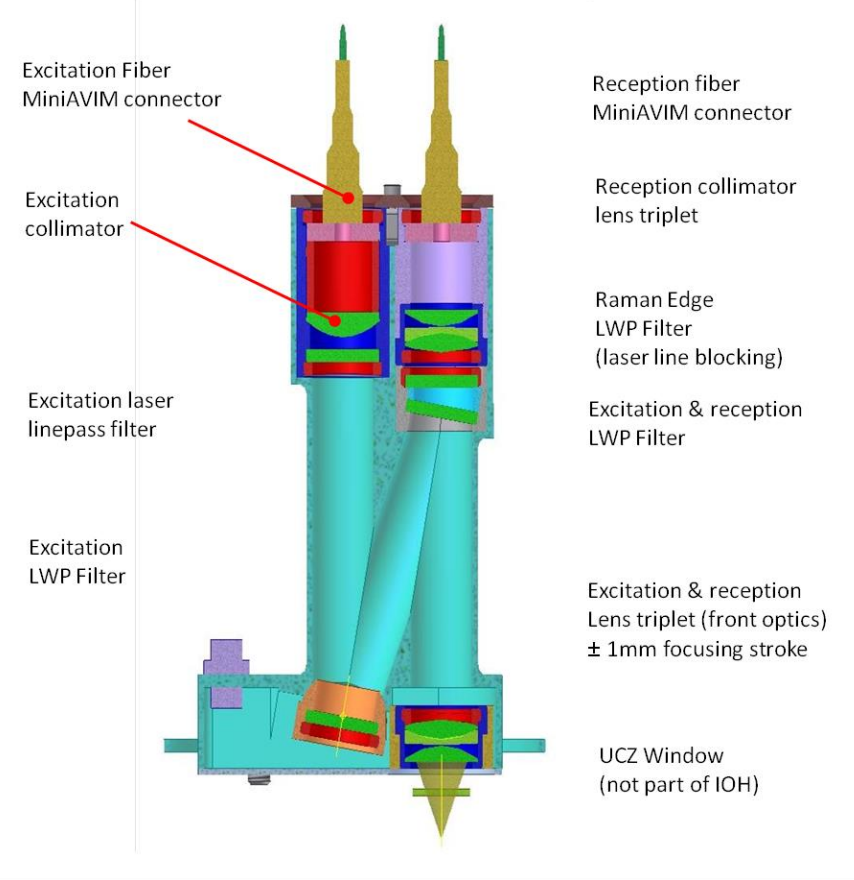

Figure 4.1. - Drawing of the IOH of RLS and its optical components. (Credit: ESA)

The IOH for the RLS instrument has been defined to have a spot diameter on the sample of $50 \mu \mathrm{m}$ at its focused position. This $50 \mu \mathrm{m}$ spot is measured at $13.5 \%$ of the maximum intensity of the laser energy distribution profile.

With respect to the movement of the sample relative to the IOH, the PSHS does the sample positioning while the only movement that the IOH has in RLS is internal and associated to the autofocus (AF) mechanism.

For the RLS-ExoMars Science Simulator, as the RC remains static in the $R L S$ ExoMars Simulation Chamber, the Raman Optical Head (OH) has to be moved in the three axis: $\mathrm{X}$ and $\mathrm{Y}$ for accessing the whole surface of the $\mathrm{RC}$, and $\mathrm{Z}$ for focusing on each spot of the sample. 


\subsection{Background: ExoMars RLS Simulator}

The RLS-ExoMars Science Simulator is based on the previous version of this simulator built as well in the Unidad Asociada UVa-CSIC, to test the operation mode of the RLS instrument: the ExoMars RLS Simulator. This instrument is shown in Figure 4.2.
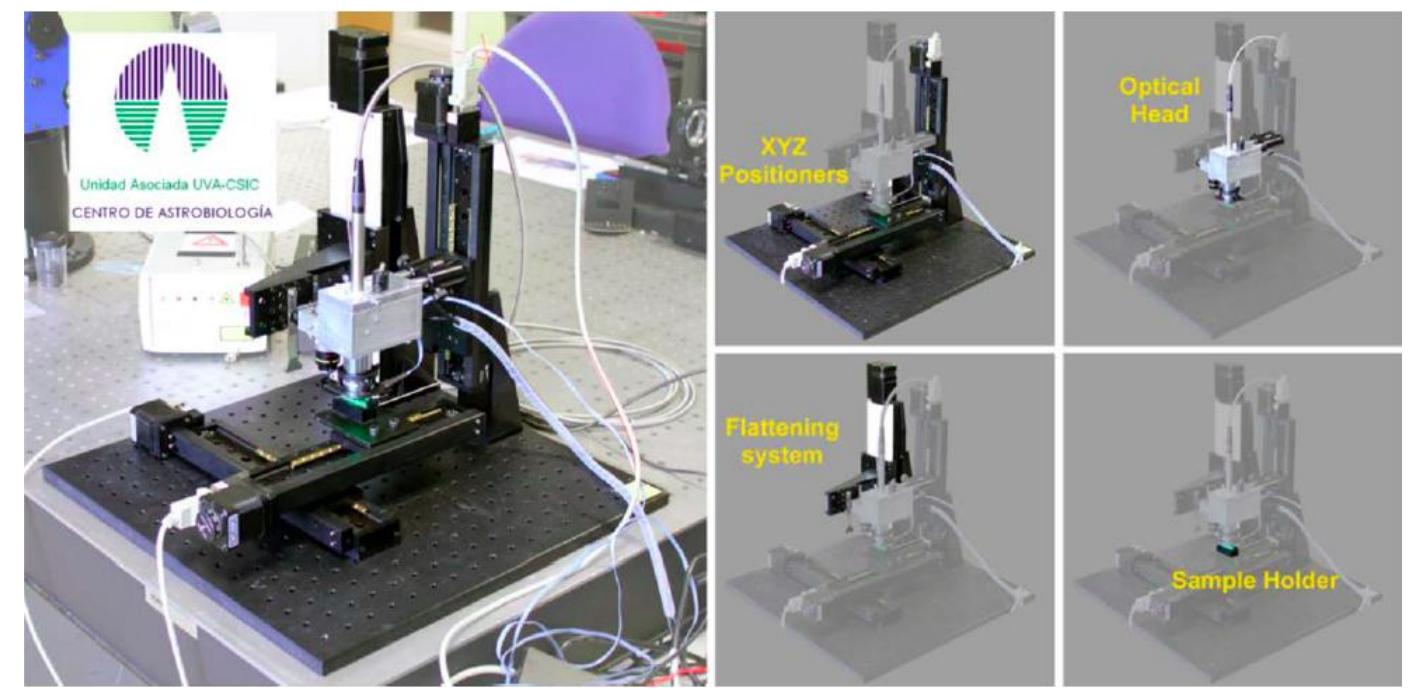

Figure 4.2. ExoMars RLS Simulator and parts (from López-Reyes et al., 2013).

As it can be noticed from Figure 4.2, the ExoMars RLS Simulator operates under laboratory environmental conditions, which is irrelevant to its task. The RC, called Sample Holder in this figure, is capable of moving in the plane of the optical bench $(\mathrm{XY})$ thanks to two linear translators by Standa. The large range of displacement of the stages is useful for performing even the flattening operation, by means of a blade attached to an adjacent vertical linear stage adjusted to the height of the sample holder within this assembly. The $\mathrm{Z}$ movement of the XYZ translation stage system is used for the autofocus operations.

The previous paragraph describes the mechanical part of this Science Simulator. Now is the turn of the even more important optical section, the Optical Head. The Optical Head $(\mathrm{OH})$ on the ExoMars RLS Simulator is comprised of the Raman probe, provided by a commercial manufacturer, adapted to an optical box containing a beamsplitter, an achromatic doublet and an imaging sensor with its associated electronics. Attached to the optical box, on the bottom part, a microscope objective serves as the optical element to focus and collect the light onto and from the sample, respectively. 
Another function of this microscope objective is not shared by the IOH on RLS but it is useful for the scientific team to gather more information about the sample and the acquisition process. It serves in combination with the imaging sensor and the doublet to take visible images of the area where the analysis spot lies.

Taking these visual images accomplishes to objectives before and after Raman acquisitions: (1) serving as the base for a visual autofocus system both manually and automatic, to select the best focus position on the region of the spot for acquiring the near-to-best Raman spectrum. (2) To visually assess the effects of the Raman laser exposure to the analysed sample.

This ExoMars RLS Simulator works under laboratory environmental conditions and it is mainly devoted to the definition and test of the operation mode of the real instrument. The operation mode is the scientific protocol to be executed every experimental cycle, which are in turn divided into as many measurement cycles as points to be analysed on a sample. The measurement cycle involves tasks such as calculation of the acquisition parameters, protocols for reducing fluorescence or eliminating cosmic rays, acquisition of spectra with the previously calculated integration time and number of accumulations for obtaining a certain signal-to-noise ratio (SNR), and post-processing operations for detecting spectral bands of interest for the instrument's scientific objectives.

With the RLS-ExoMars Simulation Chamber and the new RLS-ExoMars Science Simulator, the operation mode can be performed at a new level, under more realistic environmental conditions. 


\subsection{RLS-ExoMars Science Simulator}

The RLS-ExoMars Science Simulator is the best complementary instrument for the RLS-ExoMars Simulation Chamber presented in Chapter 3. It could be said that this version of the science simulator is an evolution of its predecessor, in the sense that it incorporates improvements to reduce its final volume and enhance operations (regardless of the application).

Figure 4.3 shows a view of the complete assembly where the science simulator can be seen.

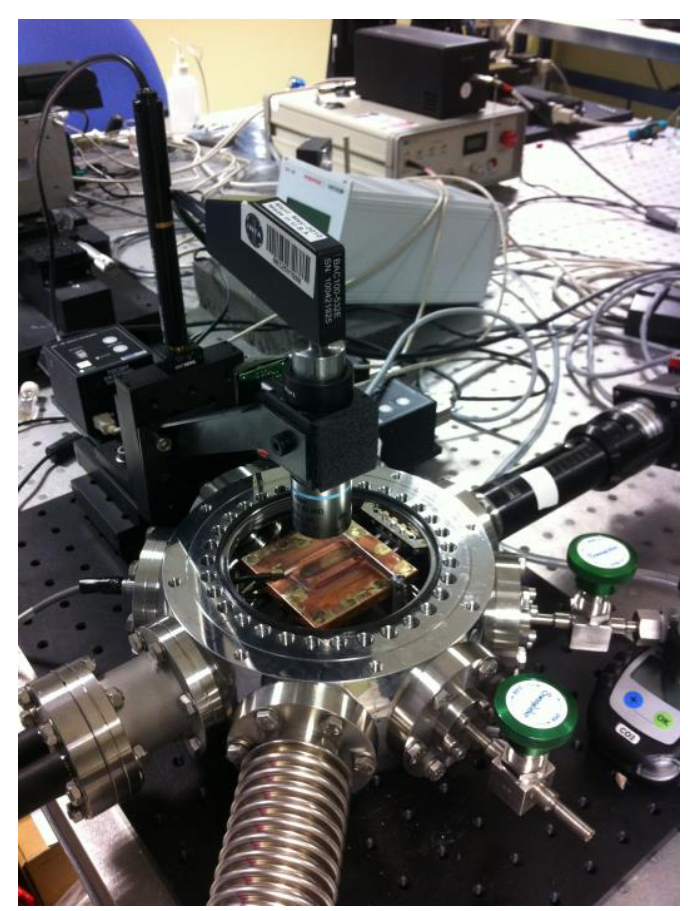

\section{Figure 4.3. - RLS-ExoMars Simulation Chamber + RLS-ExoMars Science Simulator}

To understand the parts that comprise the RLS-ExoMars Science Simulator, they are explained individually in the next subsections.

\subsubsection{XYZ positioning system}

The XYZ positioning system that has been incorporated to this science simulator for performing the task of moving the $\mathrm{OH}$ over the sample inside the simulation chamber is shown in Figure 4.4 as presented by its manufacturer, Thorlabs. 


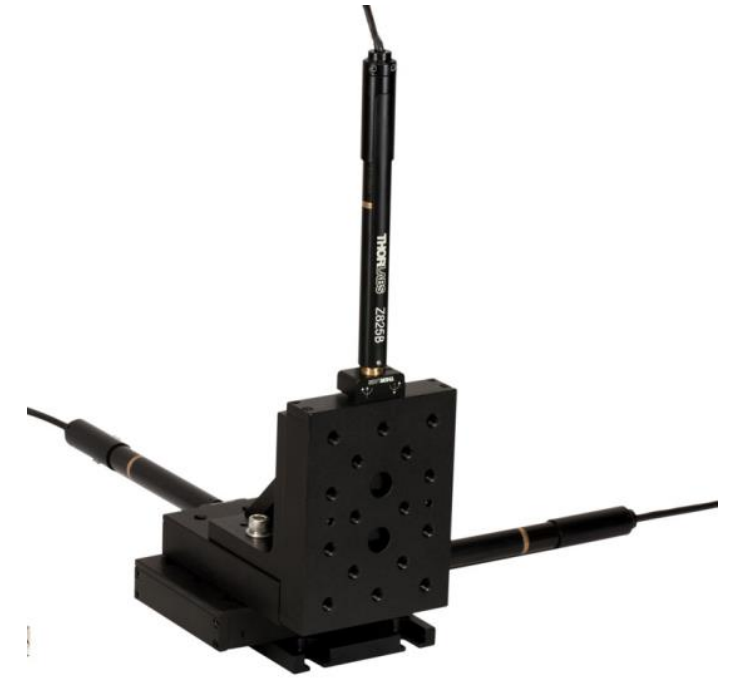

a

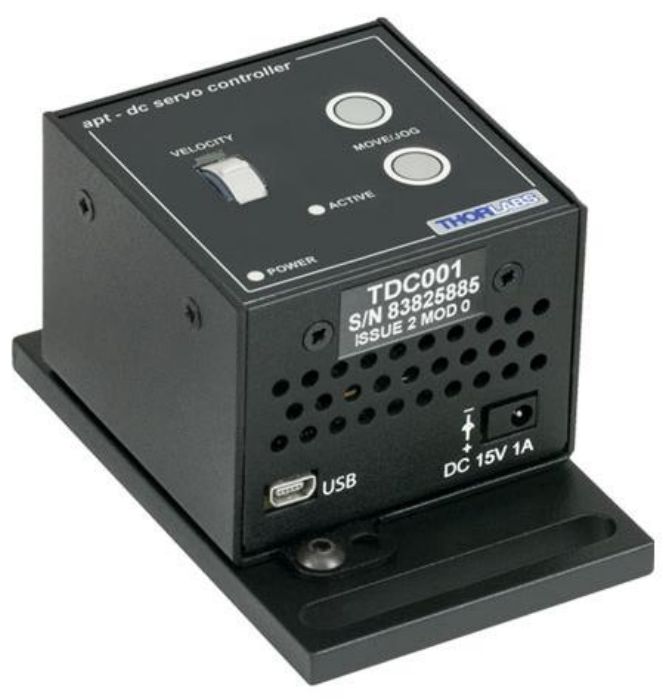

$\mathrm{b}$

Figure 4.4. - (a) PT3-Z8 XYZ motorized stage and (b) TDC001 DC servo controller. (Credit: Thorlabs, Inc.)

Each of the three linear stages that the PT3-Z8 is comprised of, is actuated by a DC Servo Actuator, in turn controlled by a TDC001 DC servo controller.

This XYZ positioner gives a range of movement in every direction of $25 \mathrm{~mm}$. This is enough to cover the entire sampling area and allow operating comfortably with the interior of the simulation chamber and performing the AF operations.

The control of these movements is made through a PC software specifically programmed for this PhD Thesis. This tool is presented in Annex A.

With respect to the previous version of this science simulator, the positioning system has been reduced considerably in volume and has gained resolution (theoretically from $0.31 \mu \mathrm{m}$ to $29 \mathrm{~nm}$ ), quietness and precision in the positioning of the $\mathrm{OH}(<1.5 \mu \mathrm{m}$ of bidirectional repeatability compared to the previous $2.5 \mu \mathrm{m})$.

\subsubsection{Optical system}

As it has been succinctly presented before, the optical system is the equivalent optical box that was described for the ExoMars RLS Simulator. It is comprised of the optical elements for accommodating the laser light to produce the $50 \mu \mathrm{m}$ spot on the sample and receiving the Raman light back to the 
spectrometer. It also allows for the visualization of the area of the sample, to know where the laser is hitting to focus the laser and observe its effects on the sampling point.

Figure 4.5 shows a descriptive 3D image of the optical box of the $R L S$ ExoMars Science Simulator. The selection of optical elements and adapters for external equipment gives the simulator high flexibility in a reduced volume. It goes from $50 \times 55 \times 90 \mathrm{~mm}^{3}$ of the ExoMars RLS Simulator to the $38.1 \times 56.4 \times 64.3 \mathrm{~mm}^{3}$ of this one.

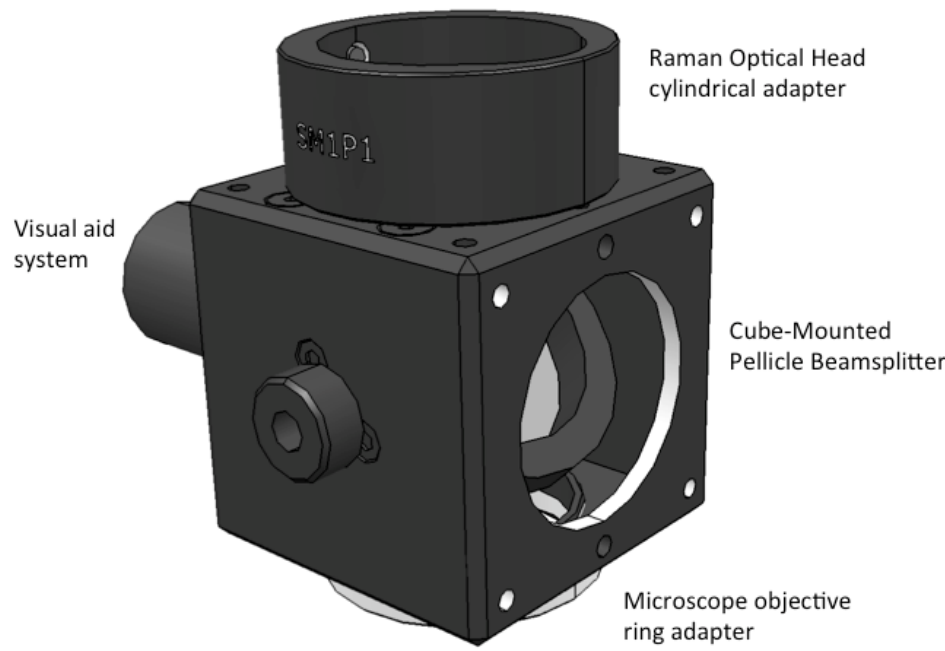

Figure 4.5. - 3D layout of the optical system of the new RLS-ExoMars Science Simulator (Credit for individual 3D parts: Thorlabs, Inc.)

\subsubsection{Cube-Mounted pellicle beamsplitter}

This optical element is used to divide the light coming from the sample in two perpendicular paths. Using Figure 4.6 as an aiding image, 92\% of the collimated light coming from the right follows the green path, which goes to the Raman $\mathrm{OH}$ for its spectroscopic analysis. The remaining 8\% goes to the perpendicular red path, which focuses on the imaging sensor to represent the scene. 


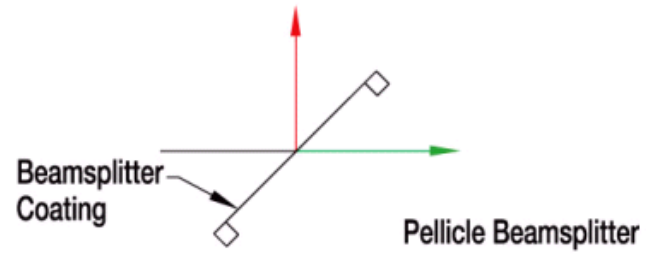

Figure 4.6. - Scheme of the pellicle beamsplitter used to divide light (Credit: Thorlabs, Inc.)

The splitting ratio has been selected in order to have the minimum loss of Raman information directed to the spectrometer, since it is a weak phenomenon. The quantity of light arriving at the imaging detector can be modulated by the amount of external incident white light on the sample during the imaging process, so an $8 \%$ is more than enough light to form an image. For obvious reasons, both spectroscopic and visual actions cannot be performed simultaneously; the white light introduced to take the image would saturate the detector at the end of the Raman spectrometer.

\subsubsection{Raman Optical Head $(\mathrm{OH})$ cylindrical adapter}

To the top side of the cube, which acts as the central body of the optical assembly, a cylindrical adapter has been screwed. This adapter allows the repeatable positioning of the Raman $\mathrm{OH}$ thanks to two retaining screws. This element can be seen in figure 4.7 .

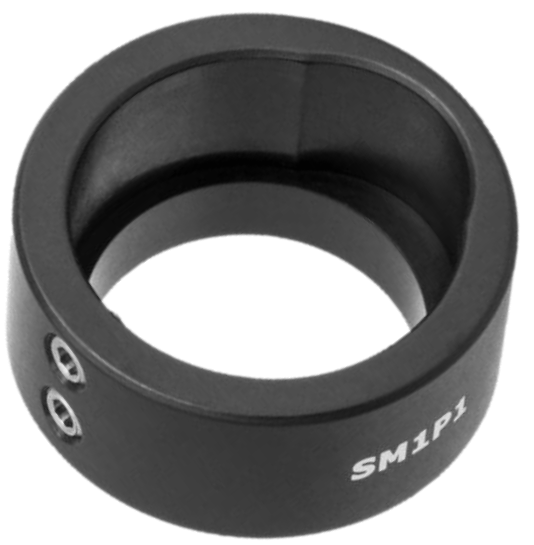

Figure 4.7. - SM1P1 by Thorlabs used as Raman $\mathrm{OH}$ cylindrical adapter (Credit: Thorlabs, Inc.)

Virtually any Raman $\mathrm{OH}$ can be attached to the optical system of this $R L S$ ExoMars Science Simulator, provided its focusing optics can be removed and it 
comes with an adaptation element to fit in this cylindrical adapter attached to the cube.

Like the actual IOH (see Figure 4.1), the currently used Raman OHs have two fibre optics attached to them. One of which connects to a laser source and the other one to a Raman spectrometer.

The specific Raman $\mathrm{OH}$ used for the tests presented at the end of this chapter and the experiments described in Chapter 5 is a BAC100-532E commercial Raman probe by BWTek designed to be used with 532nm excitation sources because of the laser line rejection filter inside it. To adapt it to this optical assembly, the focusing lens of the probe was removed to have a collimated beam going out. The microscope objective substitutes this focusing function.

\subsubsection{Microscope objective ring adapter}

This element, presented in Figure 4.8 and attached to the bottom side of the cube, allows the screwing of M25x0.75 microscope objectives.

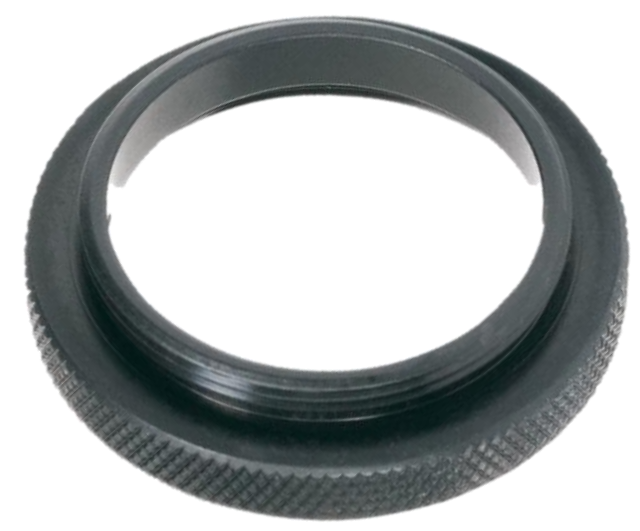

Figure 4.8. - SM1A12 ring adapter for M25x0.75 microscope objectives (Credit: Thorlabs, Inc.)

Given the fact that several of these adapters exist in the market, in the case any other type of microscope objective is decided to be used, it is only one adapter away.

The only restriction applied to them is a long working distance of $17 \mathrm{~mm}$ or more. This is approximately the distance (adding some margins for the autofocus 
operation and the sample surface irregularities) to the surface of the RC through the optical window of the RLS-ExoMars Simulation Chamber.

\subsubsection{Visual aid system}

As it has been said previously, although the actual RLS instrument does not contemplate the use of a camera to take images of the area under analysis in the visible range (MicrOmega will take a sort of them in the IR), for both the ExoMars RLS Simulator and the RLS-ExoMars Science Simulator its usefulness was considered.

Attending to figure 4.6, which explained the beamsplitter mechanism, the red path is destined to the image acquisition. The collimated light coming from the microscope objective is focused onto a webcam imaging CMOS sensor by means of an achromatic doublet inserted inside a tube screwed to the corresponding face of the cube. Figure 4.9 shows this assembly.

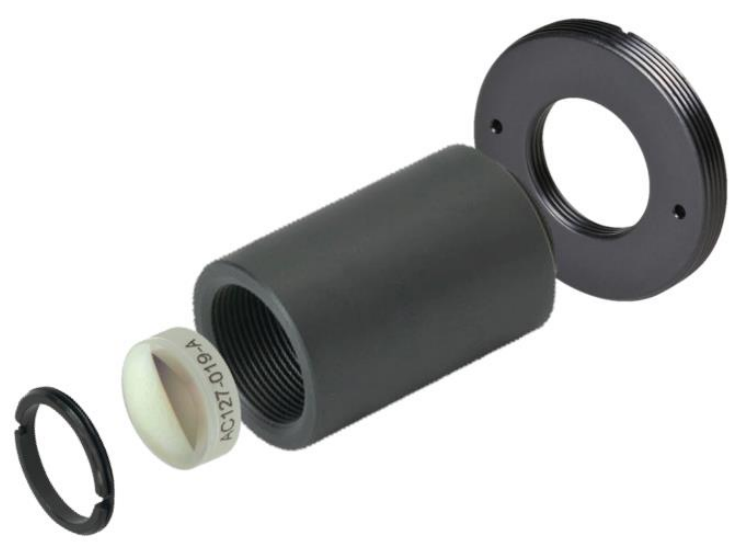

Figure 4.9. - Visual aid system optical assembly (Credit of the images for the individual components: Thorlabs, Inc.)

The achromatic doublet is placed at the correct distance inside the tube by means of two retaining rings such as the one shown at the leftmost bottom corner of Figure 4.9 in order to project the focused image of the scene onto the imaging sensor attached to the furthest-from-the-cube edge of the tube. 


\subsection{Tests}

This section presents the specific instruments and optical elements used in the experiments that will be explained in Chapter 5 and the tests performed to assess: (1) experimental variables such as laser power output at the end of the optical path, spot sizes at different heights from the focusing point; (2) instrumental capabilities such as the Depth Of Field (DOF) of the microscope objective, the spectral resolution achieved with the spectrometer + detector, and the optical system transfer function; and (3) methodological steps to calibrate the acquired spectra in wavelength and intensity.

\subsubsection{Laser power output}

This test presents the laser output power at the end of the excitation path as a function of the power regulation values (Figure 4.10 and Figure 4.11).

The laser used for this test was the 532nm BWN by BWTek capable of providing a maximum of $100 \mathrm{~mW}$ at the output of its emission port. However, the output power at the end of the excitation path gets reduced due to losses along the way.

The excitation path in the first case (Figure 4.10) is comprised of (1) a fibre optic pigtail connected to (2) the excitation fibre of the BAC100-532E Raman Probe by BWTek. This probe is inserted in the cylindrical adaptor on the top of the RLS-ExoMars Science Simulator optical assembly. The laser beam traverses then (3) the beamsplitter inside the cube of the optical box, (4) the CFI60 L Plan SLWD 50x microscope objective by Nikon and (5) the optical window of the RLS-ExoMars Simulation Chamber.

For the measurement of the laser output power a S310C Thermal Power Sensor by Thorlabs was used. A set of 100 measurements was taken for each of the selected percentage values of the maximum input power (from $0 \%$ to $100 \%$ in $5 \%$ steps). The final values in Table 4.1 and their representation in Figure 4.10 are then the average of those sets of 100 measurements. The linearity of the set of measurements is quite evident. 


\begin{tabular}{|c|c|c|}
\hline $\begin{array}{c}\text { Input Power } \\
(\%)\end{array}$ & $\begin{array}{c}\text { Average } \\
\text { Output Power } \\
\text { (mW) }\end{array}$ & $\begin{array}{l}\text { Coefficient of } \\
\text { Variation (CV) }\end{array}$ \\
\hline 5 & $1.14 \mathrm{E}+00$ & $4.0 \%$ \\
\hline 10 & $2.84 \mathrm{E}+00$ & $1.5 \%$ \\
\hline 15 & $4.55 E+00$ & $1.0 \%$ \\
\hline 20 & $6.42 E+00$ & $0.8 \%$ \\
\hline 25 & $8.12 E+00$ & $0.7 \%$ \\
\hline 30 & $1.01 \mathrm{E}+01$ & $0.6 \%$ \\
\hline 35 & $1.18 \mathrm{E}+01$ & $0.5 \%$ \\
\hline 40 & $1.35 \mathrm{E}+01$ & $0.4 \%$ \\
\hline 45 & $1.54 \mathrm{E}+01$ & $0.4 \%$ \\
\hline 50 & $1.71 \mathrm{E}+01$ & $0.4 \%$ \\
\hline 55 & $1.88 \mathrm{E}+01$ & $0.3 \%$ \\
\hline 60 & $2.07 E+01$ & $0.3 \%$ \\
\hline 65 & $2.27 \mathrm{E}+01$ & $0.5 \%$ \\
\hline 70 & $2.42 E+01$ & $0.5 \%$ \\
\hline 75 & $2.63 \mathrm{E}+01$ & $0.4 \%$ \\
\hline 80 & $2.81 \mathrm{E}+01$ & $0.7 \%$ \\
\hline 85 & $2.99 \mathrm{E}+01$ & $0.6 \%$ \\
\hline 90 & $3.18 \mathrm{E}+01$ & $0.6 \%$ \\
\hline 95 & $3.36 \mathrm{E}+01$ & $0.4 \%$ \\
\hline 100 & $3.53 E+01$ & $0.4 \%$ \\
\hline
\end{tabular}

Table 4.1. - Average output power values as a function of the input power

The coefficient of variation gives the relative standard deviation expressed as a per cent. It is calculated as: $C V=\frac{S}{\bar{X}} 100 \%$, where $s$ is the standard deviation of the measurements and $\bar{X}$ the average. 


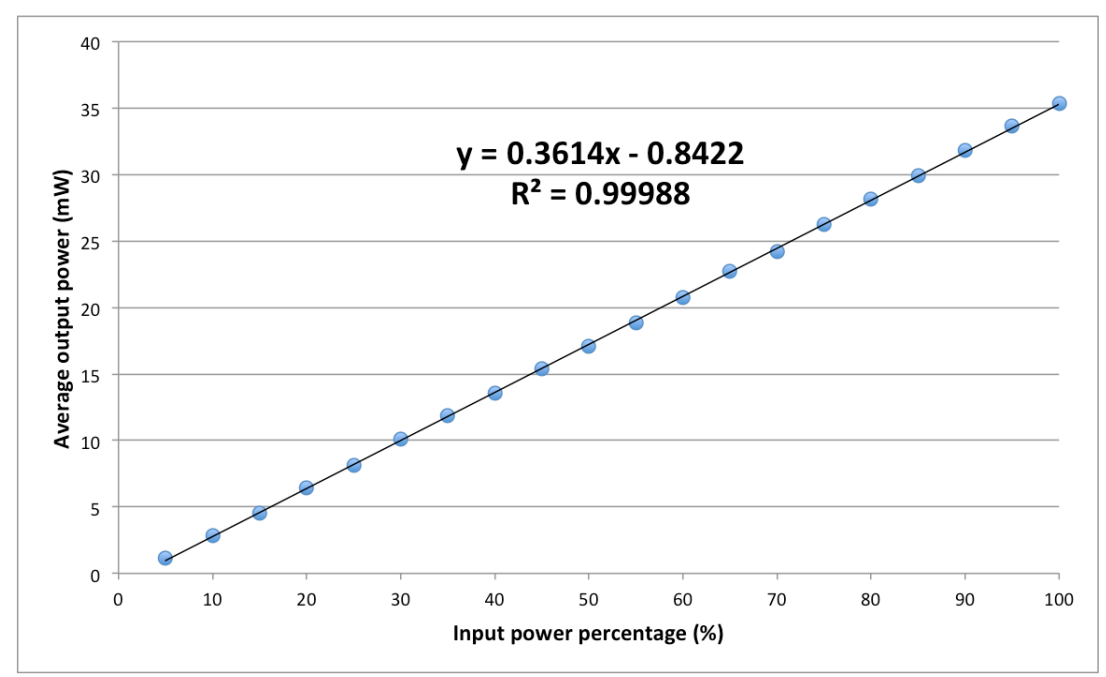

Figure 4.10. - Average output power as a function of the percentage of the maximum input power, from $0 \%$ to $100 \%$ in $5 \%$ steps.

This test will be useful in the determination of the irradiance values presented in section 4.4.3.

\subsubsection{Laser spot size}

This test aims to measure the spot size of the laser projected by the optical system of the RLS-ExoMars Science Simulator at different heights from focus. This will allow the irradiance calculation for different spot sizes, which will be used in the experiments performed in the next chapter.

The spot measurement was done illuminating the Laser Beam Profiler (LBP-2-USB) by Newport. In order to attenuate the laser light and avoid saturating or even damaging the image detector, a set of neutral density filters were placed in the optical path. They were used in combination with the electronic shutter of the measuring instrument.

The excitation optical path is the same as for the power measurement, including the optical window, to take into account the modification of this path in terms of height from focus.

Table 4.2 lists the spot size values measured at different heights and for the optical path that includes the 50x microscope objective. Each value corresponds to the average of 20 measurements. These values are measured as the full width of 
the laser profile where the intensity decreases to a $13.5 \%$ or $1 / \mathrm{e}^{2}$ of its maximum as defined by the scientific requirements (Pérez, 2013).

\begin{tabular}{|c|c|c|}
$\begin{array}{c}\text { Height from } \\
\text { focus } \\
(\mu \mathrm{m})\end{array}$ & $\begin{array}{c}\text { Spot diameter } \\
@ 13.5 \% \\
(\mu \mathrm{m})\end{array}$ & $\begin{array}{c}\text { Spot diameter } \\
@ 13.5 \% \\
\text { (gaussian fitting) } \\
(\mu \mathrm{m})\end{array}$ \\
\hline 0 & 53.39 & 56.35 \\
\hline 50 & 57.47 & 61.8 \\
\hline 100 & 69.54 & 71.91 \\
\hline 150 & 88.54 & 89.85 \\
\hline 200 & 111.12 & 109.36 \\
\hline 250 & 132.08 & 131.6 \\
\hline 300 & 155.46 & 156.6 \\
\hline 350 & 179.24 & 188.89 \\
\hline 400 & 200.05 & 210.63 \\
\hline 450 & 227.15 & 232.33 \\
\hline 500 & 250.5 & 260.85 \\
\hline 550 & 271.06 & 272.6 \\
\hline 600 & 296.03 & 293.69 \\
\hline 650 & 319.02 & 326.5 \\
\hline 700 & 345.95 & 358.73 \\
\hline 750 & 373.23 & 397.15 \\
\hline 800 & 396.52 & 426.51 \\
\hline
\end{tabular}

Table 4.2. - Laser spot diameter (measured and fitted) as a function of the height from focus

The minimum spot size is obviously reached in focus and has $\sim 50 \mu \mathrm{m}$. Figure 4.11 shows graphically these values and adjusts them to a second-degree polynomial, which adjusts better to the data as the height from focus increases. 


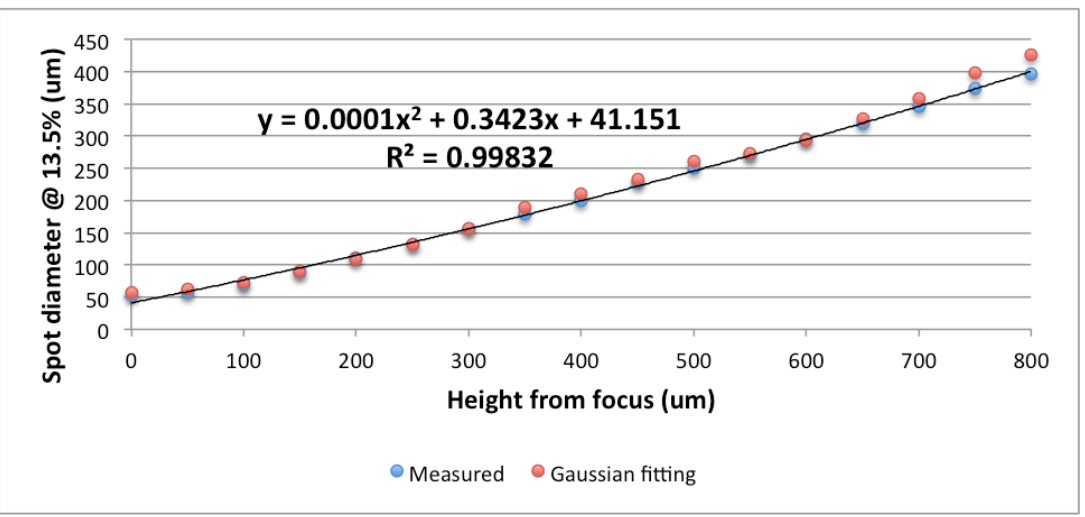

Figure 4.11. - Graph presenting the data collected in Table 4.2

To see the look of the spot in focus, Figure 4.12 shows a screen capture of the software used for this beam profile measurement.

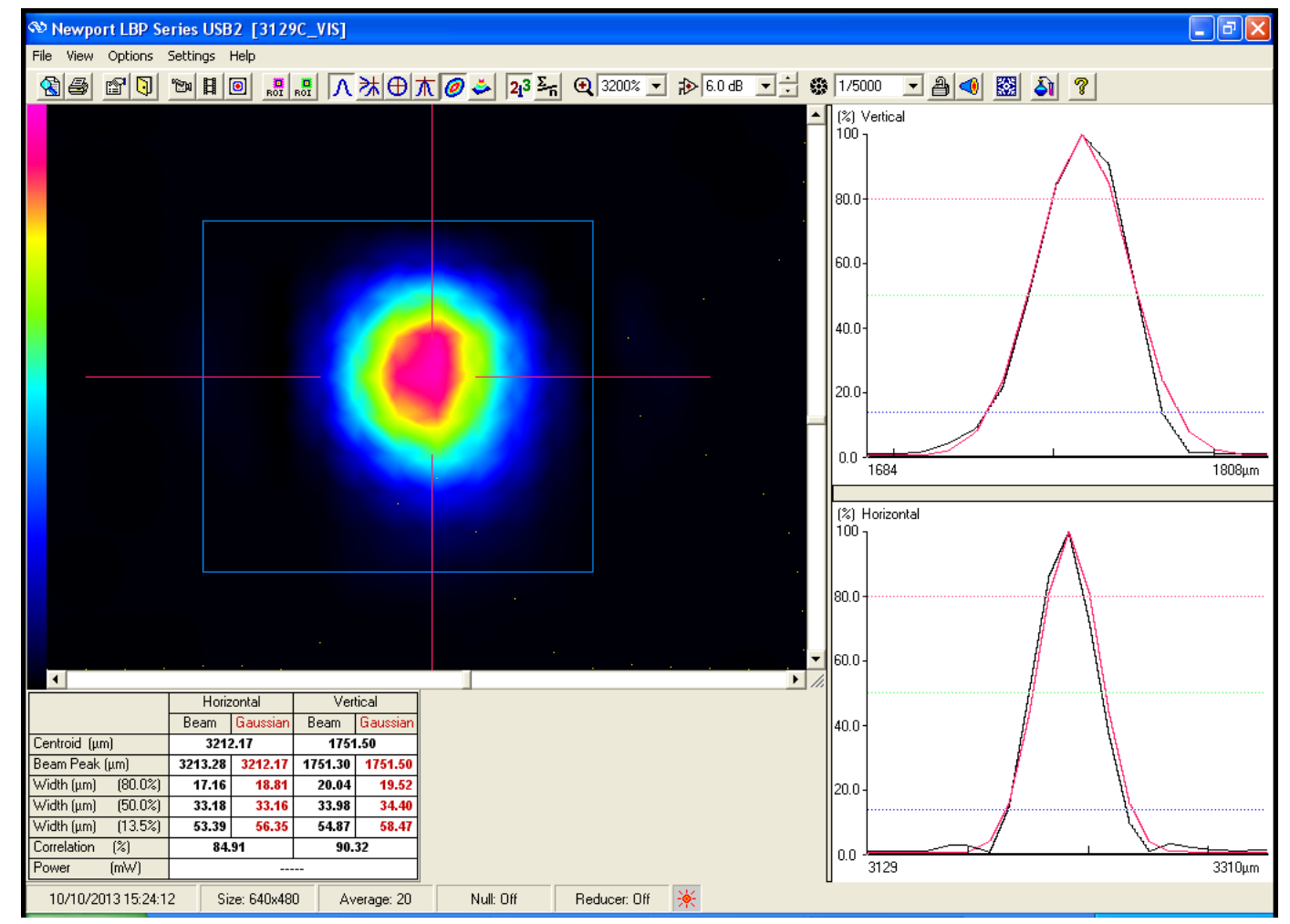

Figure 4.12. - Screenshot of the software for measuring the laser beam profile during the measurement of the spot in focus

This software shows a color-coded image of the laser spot, a right panel with the laser beam profile in two directions and the statistics associated to the measurement. 


\subsubsection{Laser irradiance}

The laser irradiance is a parameter that informs about the power density the sample is receiving, in average, inside the spot diameter.

Once the laser output power and the spot size has been measured, the laser irradiance (I) can be directly calculated using this formula:

$$
I=E_{e}=\frac{P(k W)}{\pi\left(\frac{D(c m)}{2}\right)^{2}}\left[\frac{k W}{c m^{2}}\right]
$$

In this equation $P$ is the laser output power expressed in kilowatts and $D$ is the spot diameter (or spot size) expressed in centimetres.

The following Table 4.3 presents these calculations for all the heights and laser output powers previously measured.

As with the previous measurements, these values are taken into account during the experiments performed in the next Chapter 5. 


\begin{tabular}{|c|c|c|c|c|c|c|c|c|c|c|c|c|c|c|c|c|c|c|c|c|c|}
\hline \multirow{2}{*}{$\begin{array}{c}\mathbf{H} \\
\text { (um) }\end{array}$} & \multirow{2}{*}{$\begin{array}{c}\text { D } \\
\text { (um) }\end{array}$} & \multicolumn{20}{|c|}{ Input power (\%) } \\
\hline & & 5 & 10 & 15 & 20 & 25 & 30 & 35 & 40 & 45 & 50 & 55 & 60 & 65 & 70 & 75 & 80 & 85 & 90 & 95 & 100 \\
\hline \multirow{2}{*}{0} & \multirow{2}{*}{53.39} & 5.10 & 1.27 & 2.03 & 2.87 & 73.63 & 4.52 & 5.29 & 6.05 & 6.89 & 7.63 & 8.41 & 9.26 & 1.01 & 1.08 & \begin{tabular}{|l|l|}
3 & 1.17 \\
\end{tabular} & \begin{tabular}{l|l}
7 & 1.26
\end{tabular} & 1.34 & 1.42 & \begin{tabular}{|l|l|} 
& 1.50
\end{tabular} & 1.58 \\
\hline & & $\mathrm{E}-02$ & E-01 & $\mathrm{E}-01$ & $E-01$ & $1 \mathrm{E}-01$ & $\mathrm{E}-01$ & $\mathrm{E}-01$ & $\mathrm{E}-01$ & E-01 & $E-01$ & $\mathrm{E}-01$ & $E-01$ & $E+00$ & $E+00$ & $\mathrm{D}+00$ & $E+00$ & $E+00$ & $E+00$ & $0 \mid E+00$ & $E+00$ \\
\hline \multirow{2}{*}{50} & \multirow{2}{*}{57.47} & 4.40 & 1.09 & 1.75 & 2.48 & 3.13 & 3.90 & 4.56 & 5.22 & 5.95 & 6.58 & 37.26 & \begin{tabular}{|l|}
7.99 \\
\end{tabular} & 8.75 & 9.33 & \begin{tabular}{l|l|l}
3 & 1.01
\end{tabular} & \begin{tabular}{l|l}
1 & 1.08
\end{tabular} & 1.15 & 51.23 & \begin{tabular}{l|l}
3 & 1.30
\end{tabular} & 1.36 \\
\hline & & $\mathrm{E}-02$ & E-01 & $\mathrm{E}-01$ & $E-01$ & $1 \mathrm{E}-01$ & $\mathrm{E}-01$ & $\mathrm{E}-01$ & $\mathrm{E}-01$ & E-01 & $E-01$ & $\mathrm{E}-01$ & $E-01$ & $\mathrm{E}-01$ & E-01 & $1 \mathrm{E}+00$ & $\mathrm{D} E+00$ & $E+00$ & $E+00$ & $0 \mid E+00$ & $E+00$ \\
\hline \multirow{2}{*}{100} & \multirow{2}{*}{69.54} & 3.00 & \begin{tabular}{|l}
7.47 \\
\end{tabular} & 1.20 & 1.69 & $\begin{array}{l}92.14 \\
90 .\end{array}$ & 2.66 & 3.12 & 3.57 & 4.06 & 4.50 & 4.96 & 5.46 & 5.98 & 6.37 & 76.92 & \begin{tabular}{l|l}
2 & 7.41
\end{tabular} & 7.88 & 8.38 & 8.85 & 9.30 \\
\hline & & $\mathrm{E}-02$ & E-02 & $\mathrm{E}-01$ & $\mathrm{E}-01$ & E-01 & E-01 & $\mathrm{E}-01$ & $\mathrm{E}-01$ & E-01 & $\mathrm{E}-01$ & $E-01$ & E-01 & $E-01$ & $E-01$ & E-01 & 1 $E-01$ & $\mathrm{E}-01$ & $E-01$ & 1 E-01 & E-01 \\
\hline \multirow{2}{*}{150} & \multirow{2}{*}{88.54} & 1.85 & 4.61 & 7.39 & 1.04 & 1.32 & 1.64 & 1.92 & 2.20 & 2.51 & 2.77 & 3.06 & \begin{tabular}{|l|}
3.37 \\
\end{tabular} & 3.69 & 3.93 & \begin{tabular}{|l|l}
3 & 4.27 \\
\end{tabular} & \begin{tabular}{l|l}
7 & 4.57
\end{tabular} & 4.86 & \begin{tabular}{|l|l|}
5 & 5.17 \\
\end{tabular} & \begin{tabular}{l|l}
75.46 \\
\end{tabular} & 5.74 \\
\hline & & $\mathrm{E}-02$ & E-02 & $\mathrm{E}-02$ & E-01 & E-01 & $\mathrm{E}-01$ & $\mathrm{E}-01$ & $E-01$ & E-01 & $\mathrm{E}-01$ & $E-01$ & E-01 & $E-01$ & $E-01$ & E-01 & 1 $E-01$ & $E-01$ & $E-01$ & 1 E-01 & $E-01$ \\
\hline \multirow{2}{*}{200} & \multirow{2}{*}{111.12} & 1.18 & 2.93 & 4.69 & 6.62 & 8.38 & 1.04 & 1.22 & 1.40 & 1.59 & 1.76 & 1.94 & 2.14 & 2.34 & 2.50 & 2.71 & \begin{tabular}{l|l}
1 & 2.90
\end{tabular} & 3.09 & 3.28 & \begin{tabular}{|l|l|}
8.47 \\
\end{tabular} & 3.64 \\
\hline & & E-02 & E-02 & $\mathrm{E}-02$ & $\mathrm{E}-02$ & E-02 & $\mathrm{E}-01$ & $\mathrm{E}-01$ & $E-01$ & E-01 & E-01 & E-01 & E-01 & $E-01$ & $E-01$ & $E-01$ & 1 E-01 & E-01 & $E-01$ & $1 \quad E-01$ & E-01 \\
\hline \multirow{2}{*}{250} & \multirow{2}{*}{132.08} & 8.33 & 2.07 & 3.32 & 4.69 & 95.93 & 7.38 & 8.64 & 9.88 & 1.13 & 1.25 & 1.37 & 1.51 & 1.66 & 1.77 & $\begin{array}{ll}7.92 \\
\end{array}$ & \begin{tabular}{|l|l|}
2 & 2.05
\end{tabular} & 2.19 & 2.32 & \begin{tabular}{|l|l|}
2.45 \\
2
\end{tabular} & 2.58 \\
\hline & & E-03 & E-02 & $\mathrm{E}-02$ & $\mathrm{E}-02$ & E-02 & $\mathrm{E}-02$ & $\mathrm{E}-02$ & $\mathrm{E}-02$ & E-01 & E-01 & E-01 & E-01 & $E-01$ & $E-01$ & E-01 & 1 $E-01$ & $\mathrm{E}-01$ & $E-01$ & 1 E-01 & E-01 \\
\hline \multirow{2}{*}{300} & \multirow{2}{*}{155.46} & 6.01 & 1.49 & 2.40 & 3.38 & 4.28 & 5.33 & 6.24 & 7.14 & 8.13 & 8.99 & 9.92 & 1.09 & 1.20 & 1.27 & $\begin{array}{ll}7.38 \\
7\end{array}$ & \begin{tabular}{l|l}
8 & 1.48
\end{tabular} & 1.58 & \begin{tabular}{|l|l|} 
& 1.68 \\
\end{tabular} & \begin{tabular}{|l|l|}
8 & 1.77 \\
\end{tabular} & 1.86 \\
\hline & & E-03 & E-02 & $\mathrm{E}-02$ & $\mathrm{E}-02$ & E-02 & $\mathrm{E}-02$ & $E-02$ & $\mathrm{E}-02$ & $E-02$ & $\mathrm{E}-02$ & $\mathrm{E}-02$ & $\mathrm{E}-01$ & $E-01$ & $E-01$ & E-01 & 1 E-01 & $\mathrm{E}-01$ & $\mathrm{E}-01$ & 1 E-01 & E-01 \\
\hline \multirow{2}{*}{350} & & 4.52 & 1.12 & 1.80 & 2.54 & 3.22 & 4.01 & 4.69 & 5.37 & 6.11 & 6.77 & 7.46 & 8.22 & 9.00 & 9.59 & \begin{tabular}{|l|l|} 
& 1.04 \\
\end{tabular} & \begin{tabular}{|l|l|}
4.11 \\
\end{tabular} & 1.19 & 1.26 & 51.33 & 1.40 \\
\hline & 179.24 & $\mathrm{E}-03$ & $\mathrm{E}-02$ & $\mathrm{E}-02$ & $\mathrm{E}-02$ & E-02 & $\mathrm{E}-02$ & $\mathrm{E}-02$ & $\mathrm{E}-02$ & $\mathrm{E}-02$ & $\mathrm{E}-02$ & $\mathrm{E}-02$ & $\mathrm{E}-02$ & $\mathrm{E}-02$ & $\mathrm{E}-02$ & E-01 & $1 \mathrm{E}-01$ & $\mathrm{E}-01$ & $\mathrm{E}-01$ & $1 \mathrm{E}-01$ & $\mathrm{E}-01$ \\
\hline & & 3.63 & 9.03 & 1.45 & 2.04 & 2.58 & 3.22 & 3.77 & 4.31 & 4.91 & 5.43 & 5.99 & 6.60 & 7.23 & 7.70 & 8.36 & 68.95 & 9.53 & 1.01 & \begin{tabular}{l|l}
1.07 \\
\end{tabular} & 1.12 \\
\hline J0 & S.05 & E-03 & E-03 & $\mathrm{E}-02$ & $\mathrm{E}-02$ & E-02 & $\mathrm{E}-02$ & $\mathrm{E}-02$ & $\mathrm{E}-02$ & $\mathrm{E}-02$ & $\mathrm{E}-02$ & $\mathrm{E}-02$ & $\mathrm{E}-02$ & $E-02$ & $\mathrm{E}-02$ & E-02 & $2 \mathrm{E}-02$ & $\mathrm{E}-02$ & E-01 & 1 E-01 & $\mathrm{E}-01$ \\
\hline & & 2.82 & 7.00 & 1.12 & 1.58 & 2.00 & 2.49 & 2.92 & 3.34 & 3.81 & 4.21 & 4.65 & 5.12 & 5.60 & 5.97 & 6.49 & 96.94 & 7.39 & 7.86 & \begin{tabular}{|l|l|}
6 & 8.30
\end{tabular} & 8.72 \\
\hline 0 & .15 & E-03 & E-03 & $\mathrm{E}-02$ & $\mathrm{E}-02$ & E-02 & $\mathrm{E}-02$ & $\mathrm{E}-02$ & $\mathrm{E}-02$ & $\mathrm{E}-02$ & $\mathrm{E}-02$ & $\mathrm{E}-02$ & $\mathrm{E}-02$ & $E-02$ & $E-02$ & E-02 & $2 \mathrm{E}-02$ & $\mathrm{E}-02$ & $\mathrm{E}-02$ & $2 \mathrm{E}-02$ & $\mathrm{E}-02$ \\
\hline & & 2.32 & 5.76 & 9.23 & 1.30 & 1.65 & 2.05 & 2.40 & 2.75 & 3.13 & 3.46 & 3.82 & 4.21 & 4.61 & 4.91 & 5.33 & \begin{tabular}{|l|l}
35.71
\end{tabular} & 6.08 & 6.46 & \begin{tabular}{|l|l|}
6 & 6.82
\end{tabular} & 7.17 \\
\hline 00 & 50.5 & E-03 & E-03 & $\mathrm{E}-03$ & $\mathrm{E}-02$ & $2 \mid \mathrm{E}-02$ & $\mathrm{E}-02$ & $E-02$ & $E-02$ & $E-02$ & $E-02$ & $\mathrm{E}-02$ & $E-02$ & $E-02$ & $\mathrm{E}-02$ & E-02 & \begin{tabular}{l|l}
2 & $E-02$
\end{tabular} & $E-02$ & $\mathrm{E}-02$ & \begin{tabular}{l|l}
2 & $E-02$
\end{tabular} & $\mathrm{E}-02$ \\
\hline & & 1.98 & 4.92 & 7.88 & 1.11 & 1.41 & 1.75 & 2.05 & 2.35 & 2.67 & 2.96 & 3.26 & 3.59 & 3.94 & 4.19 & 4.56 & \begin{tabular}{|l|l}
6 & 4.87
\end{tabular} & 5.19 & 5.52 & 5.83 & 6.12 \\
\hline DO & 2 & E-03 & E-03 & $\mathrm{E}-03$ & $\mathrm{E}-02$ & $2 \mathrm{E}-02$ & $\mathrm{E}-02$ & $\mathrm{E}-02$ & $\mathrm{E}-02$ & E-02 & $\mathrm{E}-02$ & $\mathrm{E}-02$ & $\mathrm{E}-02$ & $E-02$ & $E-02$ & E-02 & $2 \mathrm{E}-02$ & E-02 & $\mathrm{E}-02$ & $2 \mathrm{E}-02$ & $\mathrm{E}-02$ \\
\hline 00 & & 1.66 & 4.12 & 6.61 & 9.33 & 1.18 & 1.47 & 1.72 & 1.97 & 2.24 & 2.48 & 2.73 & 3.01 & 3.30 & 3.52 & 3.82 & \begin{tabular}{|l|l|}
2 & 4.09
\end{tabular} & 4.35 & 4.63 & \begin{tabular}{|l|l|}
3 & 4.89
\end{tabular} & 5.13 \\
\hline & & E-03 & E-03 & $\mathrm{E}-03$ & $\mathrm{E}-03$ & $\mathrm{E}-02$ & $\mathrm{E}-02$ & $\mathrm{E}-02$ & $\mathrm{E}-02$ & $E-02$ & $\mathrm{E}-02$ & $\mathrm{E}-02$ & $\mathrm{E}-02$ & $E-02$ & $E-02$ & E-02 & \begin{tabular}{|l|l|}
2 & $\mathrm{E}-00$
\end{tabular} & $\mathrm{E}-02$ & \begin{tabular}{|l|l|}
2 & $E-02$
\end{tabular} & \begin{tabular}{l|l}
2 & $E-02$
\end{tabular} & E-02 \\
\hline 50 & & 1.43 & 3.55 & 5.69 & 8.03 & 1.02 & 1.26 & 1.48 & 1.69 & 1.93 & 2.14 & 2.35 & 2.59 & 2.84 & 3.03 & 3.29 & 93.52 & 3.75 & 3.98 & \begin{tabular}{|l|l}
8 & 4.21
\end{tabular} & 4.42 \\
\hline 50 & & $\mathrm{E}-03$ & E-03 & $\mathrm{E}-03$ & $\mathrm{E}-03$ & $\mathrm{E}-02$ & $\mathrm{E}-02$ & $\mathrm{E}-\mathrm{02}$ & $\mathrm{E}-02$ & $E-02$ & $\mathrm{E}-02$ & $\mathrm{E}-02$ & $\mathrm{E}-02$ & $E-02$ & $E-02$ & E-02 & \begin{tabular}{|l|l|}
2 & $\mathrm{E}-00$
\end{tabular} & $\mathrm{E}-02$ & \begin{tabular}{|l|l|} 
& $E-02$ \\
\end{tabular} & \begin{tabular}{l|l}
2 & $E-02$
\end{tabular} & E-02 \\
\hline & & 1.21 & \begin{tabular}{|l|} 
\\
\end{tabular} & 4.84 & 6.83 & 8.64 & 1.08 & 1.26 & 1.44 & 1.64 & 1.82 & 2.00 & 2.21 & 2.42 & 2.57 & 2.80 & \begin{tabular}{l|l}
0.99
\end{tabular} & 3.19 & 3.39 & \begin{tabular}{|l|l|}
3.58 \\
\end{tabular} & 3.76 \\
\hline 0 & & E-03 & E-03 & $\mathrm{E}-03$ & $\mathrm{E}-03$ & E-03 & $\mathrm{E}-02$ & $\mathrm{E}-\mathrm{0} 2$ & $\mathrm{E}-02$ & $E-02$ & $E-02$ & $\mathrm{E}-02$ & $\mathrm{E}-02$ & $E-02$ & $E-02$ & E-02 & \begin{tabular}{|l|l|}
2 & $\mathrm{E}-02$
\end{tabular} & E-02 & \begin{tabular}{|l|l|} 
& $E-02$
\end{tabular} & \begin{tabular}{l|l}
2 & $\mathrm{E}-02$
\end{tabular} & E-02 \\
\hline & & 1.04 & 2.59 & 4.16 & 5.87 & 77.43 & 9.24 & 1.08 & 1.24 & 1.41 & 1.56 & 1.72 & 1.90 & 2.08 & 2.21 & $\begin{array}{l}1 \\
1.40\end{array}$ & 0 & 2.74 & 2.91 & $\begin{array}{l}1 \\
1\end{array}$ & 3.23 \\
\hline 0 & $=$ & E-03 & E-03 & $\mathrm{E}-03$ & $\mathrm{E}-03$ & $\mathrm{E}-03$ & $\mathrm{E}-03$ & $\mathrm{E}-02$ & $\mathrm{E}-02$ & $E-02$ & $E-02$ & $\mathrm{E}-02$ & $E-02$ & $E-02$ & E-02 & E-02 & \begin{tabular}{|l|l}
2 & $E-02$ \\
\end{tabular} & $\mathrm{E}-02$ & $E-02$ & $2 \mathrm{E}-02$ & $\mathrm{E}-02$ \\
\hline 800 & & 9.24 & 2.30 & 3.68 & 5.20 & 6.58 & 8.19 & 9.59 & 1.10 & 1.25 & 1.38 & 1.52 & 1.68 & 1.84 & 1.96 & 2.13 & \begin{tabular}{|l|l|}
3 & 2.28 \\
\end{tabular} & 2.43 & 2.58 & \begin{tabular}{|l|l|}
8.72 \\
\end{tabular} & 2.86 \\
\hline & .52 & E-04 & E-03 & $\mathrm{E}-03$ & $\mathrm{E}-03$ & E-03 & $\mathrm{E}-03$ & $\mathrm{E}-03$ & $\mathrm{E}-02$ & $\mathrm{E}-02$ & $E-02$ & $E-02$ & $\mathrm{E}-02$ & E-02 & E-02 & $\begin{array}{l}2 \mathrm{E}-02 \\
\end{array}$ & \begin{tabular}{l|l|l|}
2 & $E-02$ \\
\end{tabular} & $\mathrm{E}-02$ & E-02 & \begin{tabular}{|l|l|}
2 & $E-02$ \\
\end{tabular} & $\mathrm{E}-02$ \\
\hline & & 7.67 & 1.91 & 3.06 & 4.32 & 5.46 & 6.80 & 7.96 & 9.10 & 1.04 & 1.15 & 1.27 & 1.39 & 1.53 & 1.63 & \begin{tabular}{|l|l|} 
& 1.77
\end{tabular} & \begin{tabular}{l|l|l}
7 & 1.89
\end{tabular} & 2.01 & 2.14 & \begin{tabular}{l|l}
4 & 2.26
\end{tabular} & 2.37 \\
\hline 0 & 21 & E-04 & $\mathrm{E}-03$ & $\mathrm{E}-03$ & $\mathrm{E}-03$ & E-03 & E-03 & $\mathrm{E}-03$ & $\mathrm{E}-03$ & $\mathrm{E}-02$ & E-02 & $\mathrm{E}-02$ & $\mathrm{E}-02$ & $E-02$ & $\mathrm{E}-02$ & E-02 & \begin{tabular}{|l|l|}
2 & $E-02$ \\
\end{tabular} & $E-02$ & E-02 & \begin{tabular}{|l|l|}
2 & $E-02$ \\
\end{tabular} & E-02 \\
\hline & & 6.90 & 1.72 & 2.75 & 3.88 & 4.91 & 6.12 & 7.16 & 8.19 & 9.33 & 1.03 & 1.14 & 1.25 & 1.37 & 1.46 & 1.59 & \begin{tabular}{|l|l|}
9 & 1.70
\end{tabular} & 1.81 & 1.93 & \begin{tabular}{l|l}
3.03 \\
\end{tabular} & 2.14 \\
\hline & & $=-6$ & ? & 5 & $\mathrm{E}-03$ & $\mathrm{E}-03$ & $\mathrm{E}-\mathrm{O}$ & $\mathrm{E}-03$ & $\mathrm{E}-03$ & $\mathrm{E}-03$ & $\mathrm{E}-02$ & $\mathrm{E}-\mathrm{O} 2$ & $\mathrm{E}-02$ & $\mathrm{E}-\mathrm{O} 2$ & $\mathrm{E}-02$ & $2 \mathrm{E}-02$ & \begin{tabular}{l|l|}
2 & $\mathrm{E}-02$ \\
\end{tabular} & $\mathrm{E}-02$ & E-02 & \begin{tabular}{l|l}
$2 \mathrm{E}-02$ \\
\end{tabular} & E-02 \\
\hline
\end{tabular}

Table 4.3. - Irradiance values calculated from the spot sizes (D) at different heights $(\mathrm{H})$ and laser powers measured in the previous sections. The two cells coloured in green and red mark the current lower and upper limits of irradiance defined for the RLS instrument.

\subsubsection{Depth of Field (DOF)}

In Raman spectroscopy not only the surface in which the laser beam is focused backscatters Raman radiation. The volume of material over and below the focusing point also receives excitation radiation and will contribute to this backscattering. However not all the backscattered radiation will be collected. This volume of collection is then defined by several variables such as the opacity of the sample to the excitation radiation, the numerical aperture of the optical objective used to concentrate the laser light and the DOF. 
In photography, the DOF is defined as the interval of distances nearer and further from focus that return an acceptable sharp image. In Raman spectroscopy, we may translate this concept as the interval of distances nearer and further from focus from which an acceptable spectrum can be acquired in terms of the SNR of the Raman peaks it contains. For quantifying this 'acceptable spectrum' we will define the DOF as the interval in height where those values of SNR are greater or equal to $50 \%$ of the maximum, assumed to be reached in focus.

In order to be able to compare among optical objectives, a homogeneous good Raman scatterer has to be used. A piece of a thin silicon wafer has been selected and historically used in the Unidad Asociada UVa-CSIC to define the DOF.

Figure 4.13 presents the DOF profile for the 50x microscope objective used during these tests. They show the SNR value for the $520 \mathrm{~cm}^{-1}$ Raman peak of silicon as a function of the distance from the best focus position. This best focus is determined as that where the SNR of the cited peak is a maximum. Obviously, the acquisition parameters (integration time and number of accumulations) are kept the same for all the spectra. The SNR values are calculated using the code presented at the end of Annex C.

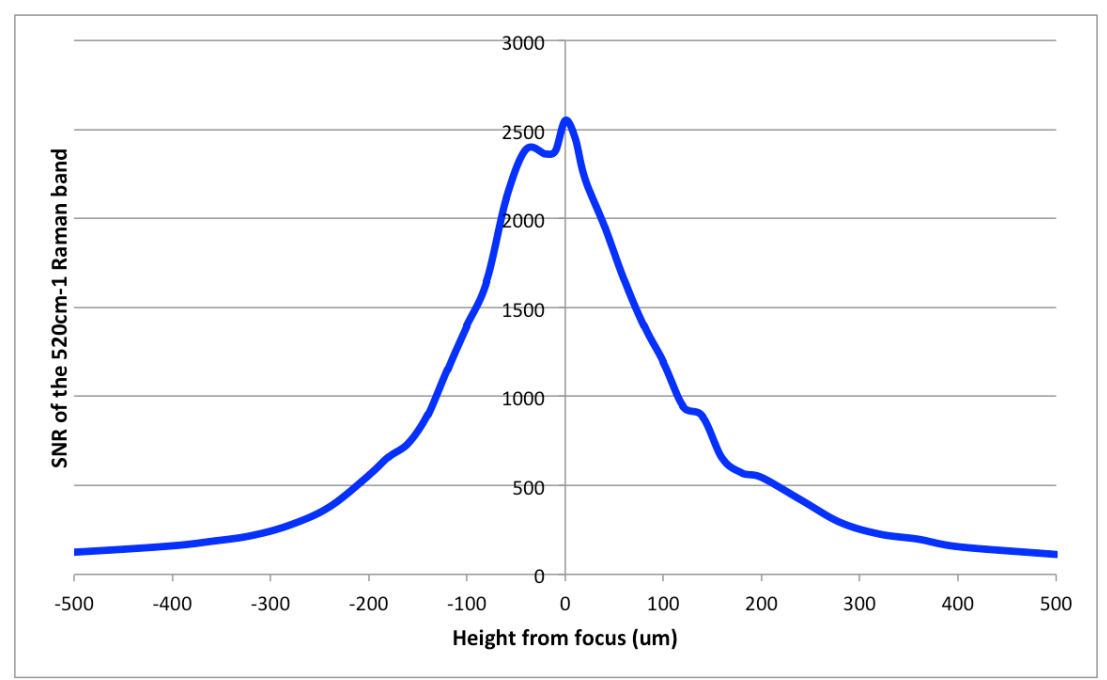

Figure 4.13. - DOF for the 50x microscope objective

The DOF value for this 50x microscope objective is, as it has been defined, $\sim 200 \mu \mathrm{m}$. 


\subsubsection{Spectral resolution}

In the specific case of these tests and the experiments presented in Chapter 5 , the spectrometer used is a Compass T (now renamed to Glacier T) by BWTek.

The size of the entrance slit, the groove density of the diffraction grating, the focal length of the optical system and the pixel size of the detector define and limit the spectral resolution of the system.

In order to quantify the resolution achieved along the spectral range of observation, the spectral widths of the atomic lines from two calibration lamps are measured. This measurement guarantees that, being atomic lines the narrowest that can be found, their width is not limited by that of the spectral peak but reflects the minimum attainable by the system. Those lamps used were a Neon lamp and an Hg lamp.

Their wavelength was firstly converted from wavelength values (in $\mathrm{nm}$ ) to Raman shift (in $\mathrm{cm}^{-1}$ ) with the wavelength calibration procedure presented in section 4.4.6. The value measured for knowing the spectral resolution of the system is the Full Width Half Maximum (FWHM). Two adjacent peaks separated a distance greater than the FWHM would be spectrally separable. To better know the FWHM of the peaks without the contribution of the discreet values provided by the individual pixels, a peak fitting to a Gaussian + Lorentzian using the curve fit algorithms of OPUS was performed.

Table 4.4 presents the measured FWHM values of the $\mathrm{Hg}$ and Neon lines falling into the spectral range of the spectrometer being used. It can be noticed that the resolution of the system ranges from $\sim 4.2 \mathrm{~cm}^{-1}$ to $\sim 6.8 \mathrm{~cm}^{-1}$, which are good values for Raman spectroscopy. The resolution defined for the RLS instrument ranges from $6 \mathrm{~cm}^{-1}$ to $8 \mathrm{~cm}^{-1}$. 


\begin{tabular}{|c|c|}
\hline Raman shift $\left(\mathrm{cm}^{-1}\right)$ & FWHM $\left(\mathrm{cm}^{-1}\right)$ \\
\hline 482 & 5.242 \\
\hline 1461 & 4.382 \\
\hline 1524 & 4.625 \\
\hline 1707 & 5.487 \\
\hline 1792 & 5.316 \\
\hline 1972 & 5.100 \\
\hline 2059 & 5.050 \\
\hline 2210 & 4.597 \\
\hline 2331 & 4.603 \\
\hline 2390 & 4.542 \\
\hline 2515 & 4.481 \\
\hline 2569 & 4.649 \\
\hline 2709 & 4.502 \\
\hline 2836 & 4.714 \\
\hline 2932 & 4.762 \\
\hline 3007 & 4.819 \\
\hline 3127 & 4.851 \\
\hline 3174 & 4.236 \\
\hline 3424 & 5.632 \\
\hline 3486 & 5.850 \\
\hline 3639 & 6.551 \\
\hline 3819 & 6.808 \\
\hline 3906 & 6.780 \\
\hline
\end{tabular}

Table 4.4. - Spectral resolutions in Raman shift for the converted peaks of the calibration lamps along the covered spectral range.

\subsubsection{Wavelength calibration}

For spectroscopy in general and for Raman spectroscopy in particular knowing the spectral position of the peaks or bands gives essential information about the molecular composition of a substance.

To know accurately these positions the spectrometer has to be previously calibrated in wavelength. This calibration consists in associating a value of Raman shift (in this particular case) to every pixel of the detector. To do this calibration lights and materials are used.

For the spectral range covered by the spectrometer being used for these tests and the experiments of Chapter 5, the calibration standards used are: (1) an Hg-Ar lamp, (2) a Neon lamp, and (3) a vial of Cyclohexane. Their spectral peaks are evenly distributed along the spectral range ( $~ 531 \mathrm{~nm}$ to $\sim 675 \mathrm{~nm})$, what provides a good calibration curve. 
The wavelength calibration algorithm programmed in Matlab is shown in Annex $\mathrm{C}$ but the general procedure is as follows:

Acquisition of spectra from the two calibration lamps in intensity versus pixel.

Acquisition of a Raman spectrum from Cyclohexane in intensity versus pixel.

Transformation from pixels to the wavelength domain by using the atomic lines emitted by calibration lamps and then transformation to the Raman shift domain using the wavelength of the excitation laser.

Polynomial fitting of the pairs of values to create the calibration curve.

Correction of the pixel-expressed spectra to Raman shift-expressed spectra.

Once the calibration curve has been calculated, the acquired spectra can be presented in Raman shift values, ready for scientific interpretation.

\subsubsection{Optical system transfer function and intensity calibration}

The elements of the collection optical path do not present a flat spectral response for the whole spectral range. Neither does the CCD detector, which has a certain Quantum Efficiency (QE) curve.

The transmission curve of the end-to-end system, including the optical window of the RLS-ExoMars Simulation Chamber, is known as the transfer function. To know it, a calibrated broadband emission light passing through the whole optical path is acquired with the spectrometer.

For obtaining this transfer function expressed in Raman shift, a wavelength calibration of both the real emission of the intensity calibration lamp and the acquired spectrum is required. In mathematical terms, the transfer function of the system $(H(k))$ is calculated from the acquired spectrum of the light passing through it $(Y(k))$ and the real broadband emission of the lamp $(X(k))$ provided by the manufacturer, as follows: 


$$
H(k)=\frac{Y(k)}{X(k)}
$$

Once the transfer function is known and has been normalized to its maximum, it can be used to calibrate the Raman spectra in intensity. This does not affect to the position of the spectral features but to its intensity, corrected from the uneven response of the system along the spectral range of observation. The normalized transfer function of the system is presented in Figure 4.14.

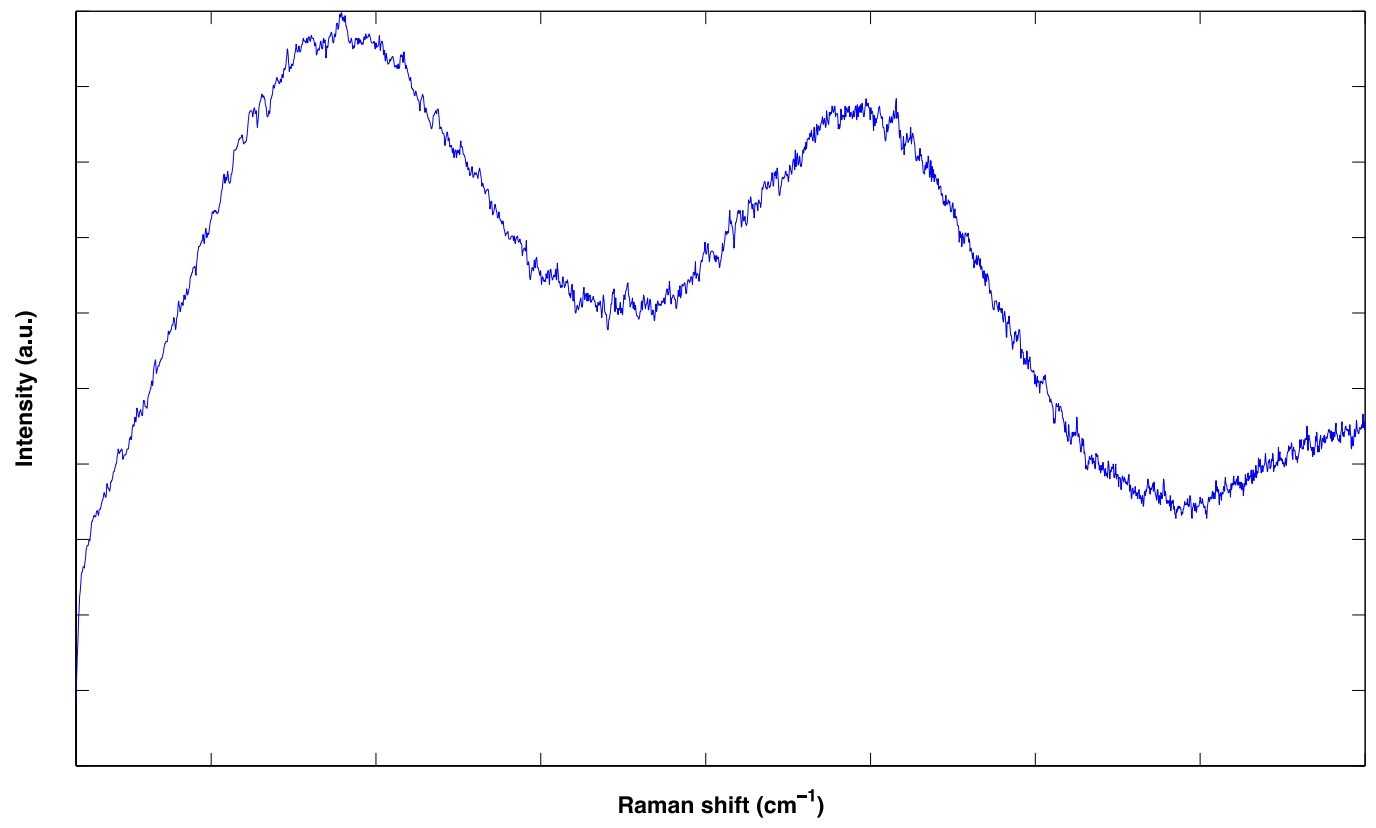

Figure 4.14. - Normalized transfer function of the system

The code presented in Annex C for wavelength calibration also incorporates intensity calibration. This intensity calibration is as simple as dividing the acquired Raman spectra $(Y(k))$ by the normalized transfer function of the system $(\bar{H}(k))$ :

$$
X(k)=\frac{Y(k)}{\bar{H}(k)}
$$

Intensity calibrated spectra allow for the study of relations among Raman peaks, which can provide more information about composition. 


\section{Chapter 5}

\section{Experiments}

The RLS-ExoMars Simulation Chamber is just a tool: a tool for science, a tool for testing the scientific requirements of the RLS instrument and the concerns regarding its operation in near-to-real environmental conditions.

This chapter presents the experiments performed on a group of representative samples under RLS-ExoMars conditions to study the spectroscopic differences compared to Earth conditions.

Part of the contents of this chapter were gathered and presented in a Technical Note made available to the RLS Team, to raise a concern regarding samples and their exposure to a laser under RLS-ExoMars conditions. 



\subsection{Introduction}

Traditionally, the databases of spectra used to interpret the results of the analyses of instruments on board robotic planetary explorers correspond to analyses performed under laboratory conditions, and in a lesser degree these spectra are taken under the mission's relevant conditions.

The main purpose in designing and building the RLS-ExoMars Simulation Chamber, is that it becomes a tool for creating new spectra databases to feed the scientific team of the RLS-ExoMars instrument.

As a preview, the first experiment presented in this chapter gathers spectra from a reduced group of representative samples, acquired at different temperatures and under RLS-ExoMars pressure conditions as well as at Earth lab conditions to compare.

As a second part of this chapter, the effects from the interaction of matter and radiation will be studied... At the beginning of the design of ESA's ExoMars mission the core sample collected from the subsurface of Mars was not planned to be crushed and delivered to the instruments as powder. The core was to be analysed as a bulk, with the purpose of obtaining not only mineralogical and chemical information of the sample itself but also knowledge about the context of its formation by means of the study of variations along the length of the core.

The scientific team of the Raman Laser Spectrometer (RLS) defined a range of irradiances to analyse this core going from $0.6 \mathrm{~kW} / \mathrm{cm}^{2}$ to $1.2 \mathrm{~kW} / \mathrm{cm}^{2}$. These irradiances were defined to be safe for the analysis of bulk samples with Raman as bulk samples have good thermal conduction or heat dissipation. The heat induced by the laser focused on a small area on the sample had little chance to affect the sample itself.

After several redefinitions of the mission, it was decided to crush the core providing the analytical instruments with powder material derived from this process. 
A concern grew around the hypothesis of thermolability of samples due to thermal effects derived from the exposure to focused laser light. That made necessary to verify whether those irradiance values were also safe for analizing powdered samples. A first test was performed on grains of selected grain size distribution (average 150-350 $\mu \mathrm{m})$ and the low irradiance value $\left(0.6 \mathrm{~kW} / \mathrm{cm}^{2}\right)$ was safe. Nevertheless, the grain size distribution of the real powdered samples delivered by the rover's crusher has proven over time to be wider with a long tail towards lower grain sizes (Tim, 2012).

The issue of thermolability prowled again, crying aloud for new tests that ensured whether the lowest irradiance value was still safe for the Raman analysis of powdered samples.

Given the fact that RLS-ExoMars relevant environmental conditions include a low atmospheric pressure (around 7 mbar), this effect gets even worse since the air convection, which could help to dissipate part of the heat produced by this exposure, is reduced.

The objective of the related experiments here presented is to confirm this hypothesis and raise the alarm on this critical issue to be further tested with the real RLS instrument in order to define a new safe range of irradiances to use in the Raman analysis of Martian samples. 


\subsection{Description of the experiments}

On one hand, in order to see how different the spectra taken under RLSExoMars relevant conditions with respect to terrestrial ones are, and have knowledge on what to expect from the real data of the mission, a collection of samples were analysed through Raman spectroscopy at different temperatures under Martian pressure and gas composition inside the RLS-ExoMars Simulation Chamber.

On the other hand, for studying the effect of exposing samples to laser radiation, two experiments were planned:

The first one focused on the progressive increase in laser output power maintaining the spot size. This allows observing the effects produced on the sample as a function of the irradiance.

The second one centred in the defocussing of the excitation area producing an increase in the spot size, maintaining the power at a fixed value: that which, at focus, gives the minimum irradiance defined for RLS-ExoMars, $0.6 \mathrm{~kW} / \mathrm{cm}^{2}$. Ideally, the collection path should be always focused on the excitation spot. Unfortunately, the optical system of the instrument is confocal, which means that defocusing the excitation also affects the collection reducing the SNR. Anyway, this experiment serves a contention solution in the case of encountering thermolabile samples even with reduced irradiance values.

These two experiments do not focus in getting good quality material, since they acquire spectra under the imposed limit of not spending more than five minutes per acquisition, but in highlighting the thermal effects that the concentrated laser excitation has on both the images and the Raman spectra.

\subsubsection{Instrumentation}

For all the experiments the instrumentation used for the setting of the environmental conditions, for the analysis of the samples, their excitation and the spectral collection and analysis was:

- $\quad$ The RLS-ExoMars Simulation Chamber presented in Chapter 3. 
- The RLS-ExoMars Science Simulator described in Chapter 4.

- BWTEK BWN 532nm 100mW laser.

- BWTEK BTC-162 Spectrometer.

- Nikon CFI L Plan SLWD 50X AN0.4 WD22mm.

\subsection{Experiment 1: Sweep in environmental conditions}

As already presented, this experiment pursues to find whether the current databases of Raman spectra acquired under laboratory conditions are valid enough to be used in the interpretation of the spectra obtained with the RLSExoMars instrument once operating on Mars, or otherwise new databases have to be built with spectra taken under RLS-ExoMars relevant environmental conditions.

For this purpose, Raman spectra were acquired from generally $-15^{\circ} \mathrm{C}$ to $5^{\circ} \mathrm{C}$ in $5^{\circ} \mathrm{C}$ steps under Martian pressure ( $\left.\sim 7 \mathrm{mbar}\right)$ and $\mathrm{CO}_{2}$ gas composition. Then, these spectra were compared among them and with spectra taken at laboratory conditions (Earth atmospheric pressure and gas composition, and $\sim 25^{\circ} \mathrm{C}$ in temperature).

\subsubsection{Methodology}

The samples were firstly placed inside the RLS-ExoMars Simulation Chamber on the Refillable Container (RC). The chamber was driven to the RLS-ExoMars environmental conditions defined as the starting point for the experiment $\left(\mathrm{P}_{\mathrm{CO} 2}=\sim 7 \mathrm{mbar}, \mathrm{T}=-15^{\circ} \mathrm{C}\right)$.

Raman spectra were taken following the following pseudocode:

Placement of the powder sample on the RC inside the RLS-ExoMars Simulation Chamber. Closing of the chamber.

Establishment of the starting environmental conditions for the experiment.

Selection of a mineral grain to analyse.

Focus

for temperature from $\min ^{\circ} \mathrm{C}$ to $\max ^{\circ} \mathrm{C}$ Acquisition of Raman spectrum.

end 
For irradiance, $0.6 \mathrm{~kW} / \mathrm{cm}^{2}$ were used in all the samples except for Jarosite, which did not accept more than $0.127 \mathrm{~kW} / \mathrm{cm}^{2}$ before degradation.

The obtained spectra have been calibrated in wavelength and intensity using the code in Annex C and the Hg-Ar and Ne lamps, and white broadband lamp. Cyclohexane has also been used as a standard to check calibration in wavelength.

\subsubsection{Samples}

A reduced group of representative samples has been analysed:

Alunite $\left(\mathrm{KAl}_{3}\left(\mathrm{SO}_{4}\right)_{2}(\mathrm{OH})_{6}\right)$ is a hydrated aluminium potassium sulphate mineral. It is an analogue of Jarosite, where the $\mathrm{Al}$ replaces the $\mathrm{Fe}^{3+}$. Alunite forms via sedimentary and low-temperature processes, and by hydrothermal activity to form veins (Bishop \& Murad, 2005).

Gypsum $\left(\mathrm{CaSO}_{4} \cdot 2 \mathrm{H}_{2} \mathrm{O}\right)$ is a sulphate mineral product, in general, of evaporitic processes. It has been identified in situ on Mars by Opportunity rover (Squyres et al., 2012).

Jarosite $\left(\mathrm{KFe}^{3+}{ }_{3}(\mathrm{OH})_{6}\left(\mathrm{SO}_{4}\right)_{2}\right)$ is a hydroxide sulphate mineral formed by the oxidation of iron sulphides and its presence is associated to low-pH waters. Jarosite was found on Mars by the Mössbauer instrument onboard NASA's Opportunity rover (Klingelhöfer et al., 2004), providing mineralogical evidence for aqueous processes on Mars.

Quartz $\left(\mathrm{SiO}_{2}\right)$ is a mineral common in sedimentary rocks as well as in igneous rocks.

In order to being realistic in terms of the ExoMars mission, these samples, as well as the ones analysed in the next subsection were prepared in powder with a grain size distribution adjusted to the critical range of the proposed distribution for the mission. $40 \%$ in mass in the range $50-125 \mu \mathrm{m}$ and $60 \%$ in the $125-250 \mu \mathrm{m}$ range, the lower part of the Gaussian distribution, the most susceptible to changes during the course of the experiments due to its volume. 


\subsubsection{Alunite}

The first analysed sample was Alunite. Figures 5.1.a and 5.1.b show a comparative of the acquired Raman spectra for this mineral and the related database spectrum under laboratory conditions, for low and high Raman shift regions, respectively.

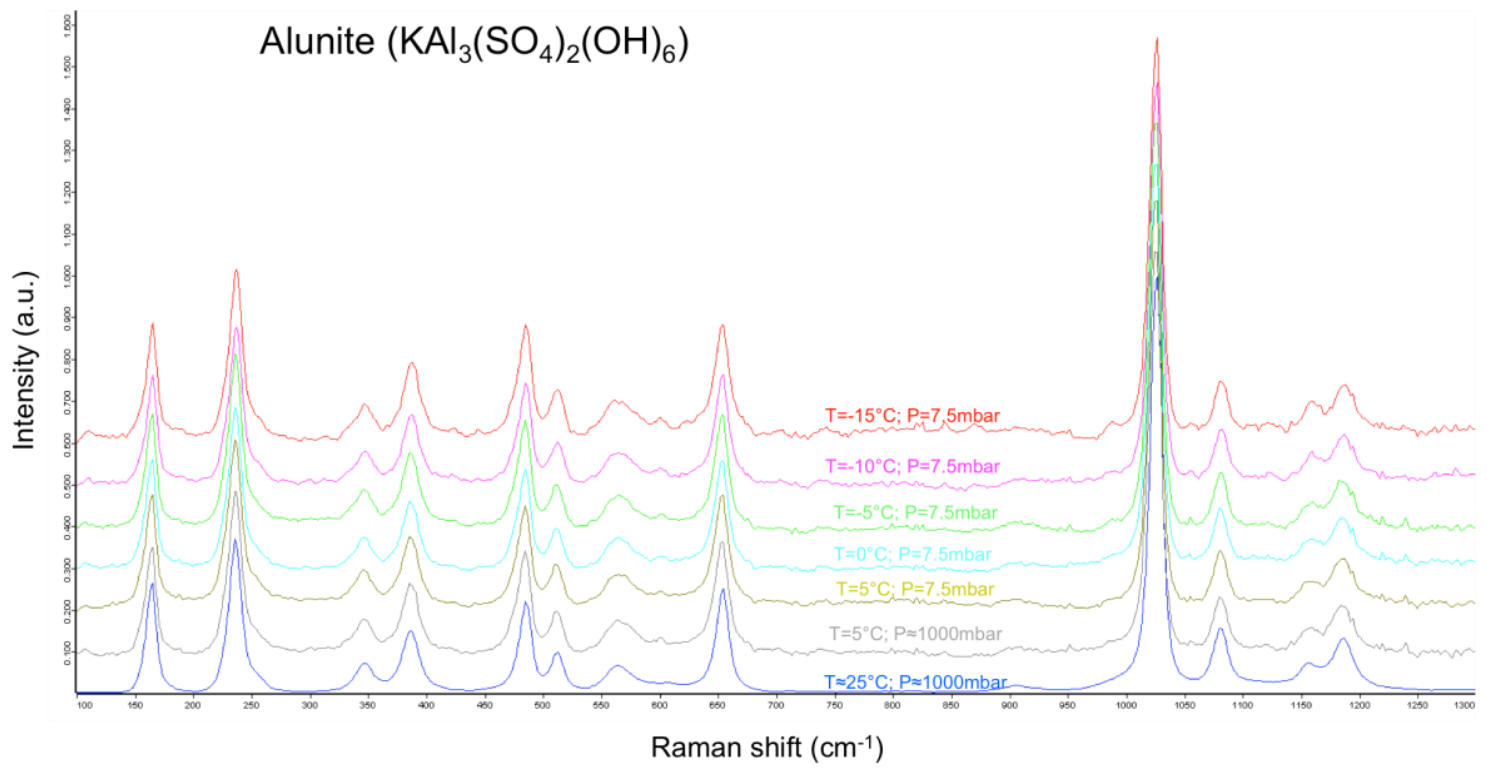

Figure 5.1.a - Low Raman shift region $\left(100-1300 \mathrm{~cm}^{-1}\right)$ of Alunite spectra taken at different environmental conditions

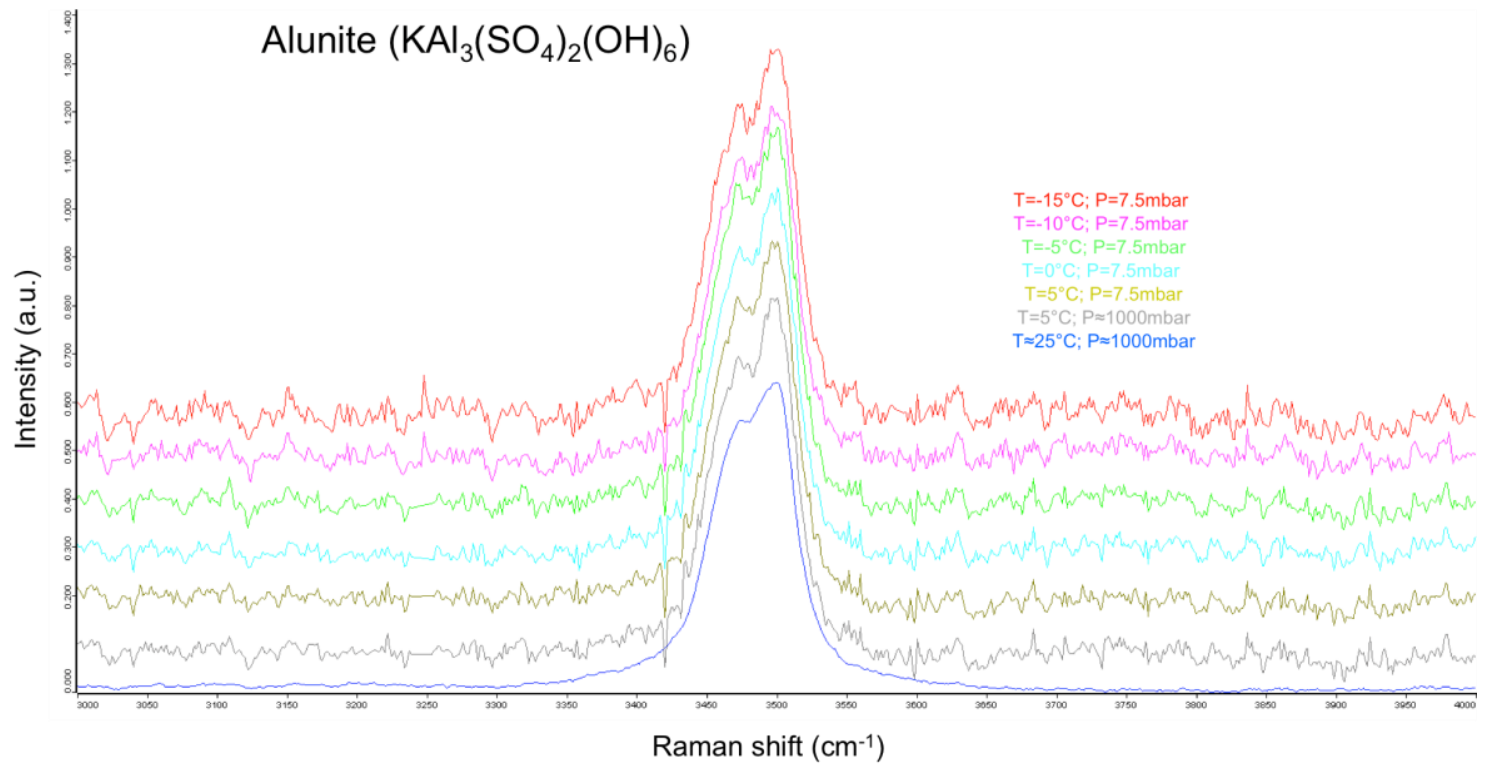

Figure 5.1.b - High Raman shift region (3000-4000 $\left.\mathrm{cm}^{-1}\right)$ of Alunite spectra taken at different environmental conditions

Figures 5.1 show no spectral changes in Alunite Raman spectra, at least at first sight. Table 5.1 presents the list of spectra with their most relevant peaks, 
which have been fitted to a Lorentz + Gauss function to approximately determine their position and width.

From Table 5.1 no trends are observed related to shifts in wavenumber or widths of the peaks although there are shifts of even $3 \mathrm{~cm}^{-1}$. These variations are in some cases produced by the poorer fitting of noisy spectra. A drastic example of this is the case of the $\mathrm{OH}^{-}$bands. 


\begin{tabular}{|c|c|c|c|c|c|c|c|c|c|c|}
\hline \multirow[b]{2}{*}{ Alunite Spectrum } & \multicolumn{2}{|c|}{$v 1$} & \multicolumn{2}{|c|}{$v 2$} & \multicolumn{2}{|c|}{ v3 } & \multicolumn{2}{|c|}{$v 4$} & \multicolumn{2}{|c|}{$\mathrm{OH}^{-}$} \\
\hline & $\begin{array}{c}\text { Pos } \\
\left(\mathrm{cm}^{-1}\right)\end{array}$ & $\begin{array}{l}\text { Width } \\
\left(\mathrm{cm}^{-1}\right)\end{array}$ & $\begin{array}{c}\text { Pos } \\
\left(\mathrm{cm}^{-1}\right)\end{array}$ & $\begin{array}{l}\text { Width } \\
\left(\mathrm{cm}^{-1}\right)\end{array}$ & $\begin{array}{c}\text { Pos } \\
\left(\mathrm{cm}^{-1}\right)\end{array}$ & $\begin{array}{l}\text { Width } \\
\left(\mathrm{cm}^{-1}\right)\end{array}$ & $\begin{array}{c}\text { Pos } \\
\left(\mathrm{cm}^{-1}\right)\end{array}$ & $\begin{array}{l}\text { Width } \\
\left(\mathrm{cm}^{-1}\right)\end{array}$ & $\begin{array}{c}\text { Pos } \\
\left(\mathrm{cm}^{-1}\right)\end{array}$ & $\begin{array}{l}\text { Width } \\
\left(\mathrm{cm}^{-1}\right)\end{array}$ \\
\hline Red: $\mathrm{T}=-15^{\circ} \mathrm{C} ; \mathrm{P}=7.5 \mathrm{mbar}$ & 1026 & 13 & $\begin{array}{l}484 \\
514\end{array}$ & $\begin{array}{l}19 \\
15\end{array}$ & $\begin{array}{l}1082 \\
1158 \\
1189 \\
\end{array}$ & $\begin{array}{l}23 \\
24 \\
32 \\
\end{array}$ & 653 & 22 & $\begin{array}{l}3478 \\
3504\end{array}$ & $\begin{array}{l}70 \\
22\end{array}$ \\
\hline Pink: $\mathrm{T}=-10^{\circ} \mathrm{C} ; \mathrm{P}=7.5 \mathrm{mbar}$ & 1026 & 13 & $\begin{array}{l}484 \\
513\end{array}$ & $\begin{array}{l}18 \\
15\end{array}$ & $\begin{array}{l}1082 \\
1158 \\
1188 \\
\end{array}$ & $\begin{array}{l}22 \\
24 \\
32 \\
\end{array}$ & 653 & 20 & $\begin{array}{l}3472 \\
3503\end{array}$ & $\begin{array}{l}53 \\
27\end{array}$ \\
\hline Green: $\mathrm{T}=-5^{\circ} \mathrm{C} ; \mathrm{P}=7.5 \mathrm{mbar}$ & 1025 & 13 & $\begin{array}{l}484 \\
513\end{array}$ & $\begin{array}{l}18 \\
15\end{array}$ & $\begin{array}{l}1081 \\
1156 \\
1186 \\
\end{array}$ & $\begin{array}{l}18 \\
20 \\
31 \\
\end{array}$ & 653 & 17 & $\begin{array}{l}3475 \\
3503\end{array}$ & $\begin{array}{l}58 \\
24\end{array}$ \\
\hline Light blue: $\mathrm{T}=0^{\circ} \mathrm{C} ; \mathrm{P}=7.5 \mathrm{mbar}$ & 1025 & 13 & $\begin{array}{l}484 \\
513\end{array}$ & $\begin{array}{l}17 \\
15\end{array}$ & $\begin{array}{l}1081 \\
1155 \\
1186 \\
\end{array}$ & $\begin{array}{l}22 \\
21 \\
35 \\
\end{array}$ & 653 & 18 & $\begin{array}{l}3471 \\
3502\end{array}$ & $\begin{array}{l}51 \\
28\end{array}$ \\
\hline Ocre: $\mathrm{T}=5^{\circ} \mathrm{C} ; \mathrm{P}=7.5 \mathrm{mbar}$ & 1025 & 13 & $\begin{array}{l}484 \\
513\end{array}$ & $\begin{array}{l}17 \\
15\end{array}$ & $\begin{array}{l}1081 \\
1155 \\
1186 \\
\end{array}$ & $\begin{array}{l}19 \\
21 \\
31 \\
\end{array}$ & 653 & 19 & $\begin{array}{l}3476 \\
3503\end{array}$ & $\begin{array}{l}60 \\
24\end{array}$ \\
\hline Grey: $\mathrm{T}=5^{\circ} \mathrm{C} ; \mathrm{P} \approx 1000 \mathrm{mbar}$ & 1025 & 13 & $\begin{array}{l}484 \\
513\end{array}$ & $\begin{array}{l}19 \\
15\end{array}$ & $\begin{array}{l}1081 \\
1155 \\
1186\end{array}$ & $\begin{array}{l}21 \\
21 \\
32 \\
\end{array}$ & 653 & 20 & $\begin{array}{l}3476 \\
3503\end{array}$ & $\begin{array}{l}63 \\
23\end{array}$ \\
\hline Dark blue: $\mathrm{T}=25^{\circ} \mathrm{C} ; \mathrm{P} \approx 1000 \mathrm{mbar}$ & 1026 & 12 & $\begin{array}{l}485 \\
513\end{array}$ & $\begin{array}{l}14 \\
14\end{array}$ & $\begin{array}{l}1081 \\
1156 \\
1187 \\
\end{array}$ & $\begin{array}{l}18 \\
25 \\
33 \\
\end{array}$ & 654 & 13 & $\begin{array}{l}3471 \\
3501\end{array}$ & $\begin{array}{l}50 \\
30\end{array}$ \\
\hline
\end{tabular}

Table 5.1. - Positions and widths of the main Raman peaks of Alunite 


\subsubsection{Gypsum}

Following with the list of samples, the next one is Gypsum. Figures 5.2.a and 5.2.b show the acquired Raman spectra in comparison with each other and with its "terrestrial" counterpart. As before, low and high Raman shifts at each subfigure.

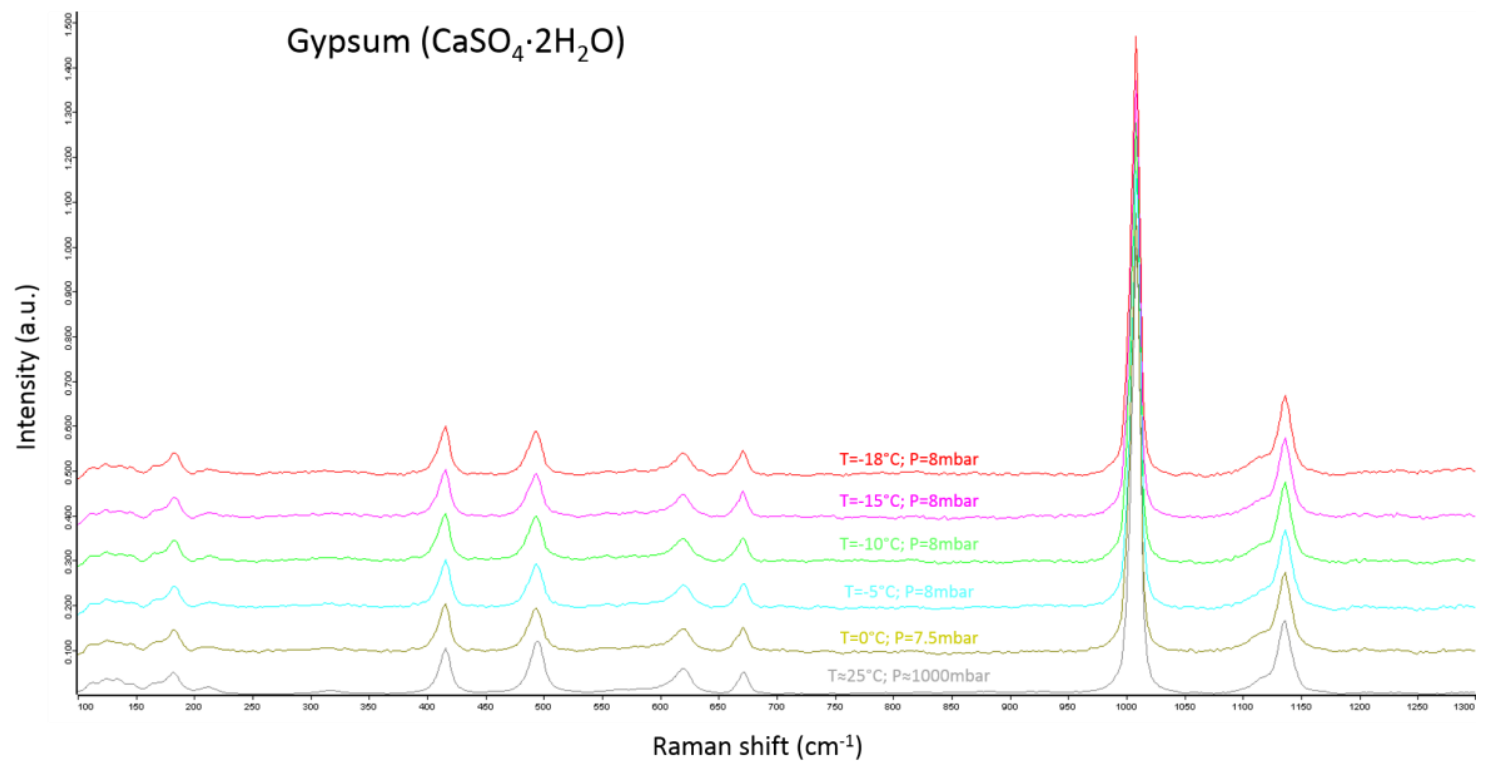

Figure 5.2.a. - Low Raman shift region (100-1300 $\left.\mathrm{cm}^{-1}\right)$ of Gypsum spectra taken at different environmental conditions

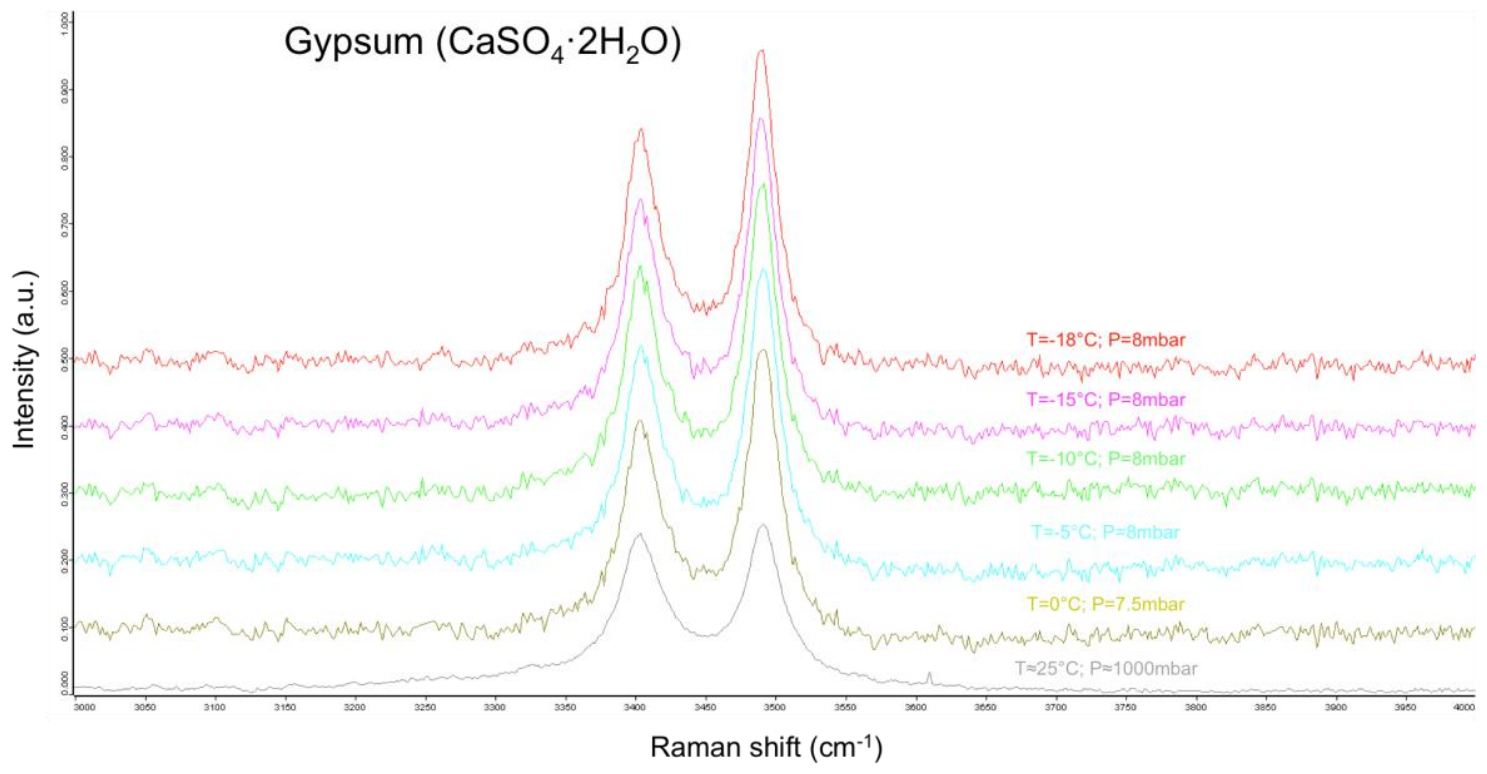

Figure 5.2.b. - High Raman shift region (3000-4000 $\left.\mathrm{cm}^{-1}\right)$ of Gypsum spectra taken at different environmental conditions

Once again, no spectral signatures are observed directly from the figure. A closer look to the peaks is presented in Table 5.2 in which the slight variations observed between spectral signatures under different environmental conditions of 
acquisition do not account for changes in the structure of the mineral produced by these conditions.

Looking more in detail at the last spectrum, the one acquired at $25^{\circ} \mathrm{C}$ and ambient pressure, a difference in the heights of the two $\mathrm{H}_{2} \mathrm{O}$ peaks presented with respect to the ones of the other spectra is noticed. The relative intensity of these peaks may be explained by either of these events:

1) Being this spectrum from the Raman spectral database, the excited point on the sample may (and does indeed) present different proportions in the orientation of water molecules (Venegas, 2014). The Raman response that is collected from different vibrations of the $\mathrm{H}_{2} \mathrm{O}$ molecules, depending on the orientation to the electric field of the laser light with respect to those molecules is producing this difference in intensity of the peaks.

2) The crushing process performed on the gypsum bulky sample has been intense enough to dehydrate part of the gypsum grains transforming them into bassanite ones. This mixture of materials could be the cause for the imbalance in the intensities of those two Raman peaks (Liu et al., 2009).

3) The effect described in 2) is produced, not as a result of the crushing process but as a dehydration of gypsum crystals inside the chamber, due to the low pressure conditions. However, the hydration and dehydration processes of gypsum are known to be slow (Chou et al., 2007; Cloutis et al., 2007)

4) The local heat produced by the concentrated laser beam in the presence of the low-pressure atmosphere inside the chamber, dehydrates the gypsum crystals and transform them into bassanite. This hypothesis is not consistent with Raman bands obtained by Prasad et al., 2001 and Prieto-Taboada et al., 2014.

Although the first hypothesis seems more plausible, further investigation has to be conducted to determine to what extent the crusher on ExoMars can alter the nature of the collected samples or how much time we have to analyse the samples before they transform due to the Martian surface conditions. 


\begin{tabular}{|c|c|c|c|c|c|c|c|c|c|c|}
\hline \multirow[b]{2}{*}{ Gypsum Spectrum } & \multicolumn{2}{|c|}{$v 1$} & \multicolumn{2}{|c|}{$v 2$} & \multicolumn{2}{|c|}{ v3 } & \multicolumn{2}{|c|}{$v 4$} & \multicolumn{2}{|c|}{$\mathrm{H}_{2} \mathrm{O}$} \\
\hline & $\begin{array}{c}\text { Pos } \\
\left(\mathrm{cm}^{-1}\right)\end{array}$ & $\begin{array}{l}\text { Width } \\
\left(\mathrm{cm}^{-1}\right)\end{array}$ & $\begin{array}{c}\text { Pos } \\
\left(\mathrm{cm}^{-1}\right)\end{array}$ & $\begin{array}{l}\text { Width } \\
\left(\mathrm{cm}^{-1}\right)\end{array}$ & $\begin{array}{c}\text { Pos } \\
\left(\mathrm{cm}^{-1}\right)\end{array}$ & $\begin{array}{l}\text { Width } \\
\left(\mathrm{cm}^{-1}\right)\end{array}$ & $\begin{array}{c}\text { Pos } \\
\left(\mathrm{cm}^{-1}\right)\end{array}$ & $\begin{array}{l}\text { Width } \\
\left(\mathrm{cm}^{-1}\right)\end{array}$ & $\begin{array}{c}\text { Pos } \\
\left(\mathrm{cm}^{-1}\right)\end{array}$ & $\begin{array}{l}\text { Width } \\
\left(\mathrm{cm}^{-1}\right)\end{array}$ \\
\hline Red: $\mathrm{T}=-18^{\circ} \mathrm{C} ; \mathrm{P}=8 \mathrm{mbar}$ & 1008 & 9 & $\begin{array}{l}414 \\
493\end{array}$ & $\begin{array}{l}19 \\
20\end{array}$ & 1137 & 11 & $\begin{array}{l}618 \\
671\end{array}$ & $\begin{array}{l}34 \\
17\end{array}$ & $\begin{array}{l}3405 \\
3490\end{array}$ & $\begin{array}{l}38 \\
27\end{array}$ \\
\hline Pink: $\mathrm{T}=-15^{\circ} \mathrm{C} ; \mathrm{P}=8 \mathrm{mbar}$ & 1008 & 9 & $\begin{array}{l}414 \\
493 \\
\end{array}$ & $\begin{array}{l}23 \\
24 \\
\end{array}$ & 1137 & 12 & $\begin{array}{l}618 \\
671 \\
\end{array}$ & $\begin{array}{l}37 \\
19 \\
\end{array}$ & $\begin{array}{l}3405 \\
3490 \\
\end{array}$ & $\begin{array}{l}43 \\
28 \\
\end{array}$ \\
\hline Green: $\mathrm{T}=-10^{\circ} \mathrm{C} ; \mathrm{P}=8 \mathrm{mbar}$ & 1008 & 9 & $\begin{array}{l}414 \\
493\end{array}$ & $\begin{array}{l}21 \\
21\end{array}$ & 1137 & 12 & $\begin{array}{l}618 \\
670\end{array}$ & $\begin{array}{l}35 \\
18\end{array}$ & $\begin{array}{l}3405 \\
3491\end{array}$ & $\begin{array}{l}41 \\
29\end{array}$ \\
\hline Light blue: $\mathrm{T}=-5^{\circ} \mathrm{C} ; \mathrm{P}=8 \mathrm{mbar}$ & 1009 & 9 & $\begin{array}{l}415 \\
493 \\
\end{array}$ & $\begin{array}{l}22 \\
23\end{array}$ & 1137 & 12 & $\begin{array}{l}618 \\
671\end{array}$ & $\begin{array}{l}37 \\
19\end{array}$ & $\begin{array}{l}3405 \\
3491\end{array}$ & $\begin{array}{l}44 \\
29\end{array}$ \\
\hline Ocre: $\mathrm{T}=0^{\circ} \mathrm{C} ; \mathrm{P}=8 \mathrm{mbar}$ & 1008 & 9 & $\begin{array}{l}414 \\
493 \\
\end{array}$ & $\begin{array}{l}19 \\
22\end{array}$ & 1137 & 12 & $\begin{array}{l}618 \\
671\end{array}$ & $\begin{array}{l}34 \\
18\end{array}$ & $\begin{array}{l}3405 \\
3491\end{array}$ & $\begin{array}{l}44 \\
29\end{array}$ \\
\hline Dark blue: $\mathrm{T}=25^{\circ} \mathrm{C} ; \mathrm{P} \approx 1000 \mathrm{mbar}$ & 1009 & 9 & $\begin{array}{l}415 \\
494 \\
\end{array}$ & $\begin{array}{l}13 \\
15 \\
\end{array}$ & 1137 & 14 & $\begin{array}{l}619 \\
672 \\
\end{array}$ & $\begin{array}{l}24 \\
12\end{array}$ & $\begin{array}{l}3404 \\
3492 \\
\end{array}$ & $\begin{array}{l}50 \\
36\end{array}$ \\
\hline
\end{tabular}

Table 5.2. - Positions and widths of the main Raman peaks of Gypsum 


\subsubsection{Jarosite}

The third relevant sample analysed under these conditions was Jarosite.

Figures 5.3.a and 5.3.b show a comparative of the acquired Raman spectra and the corresponding database spectrum.

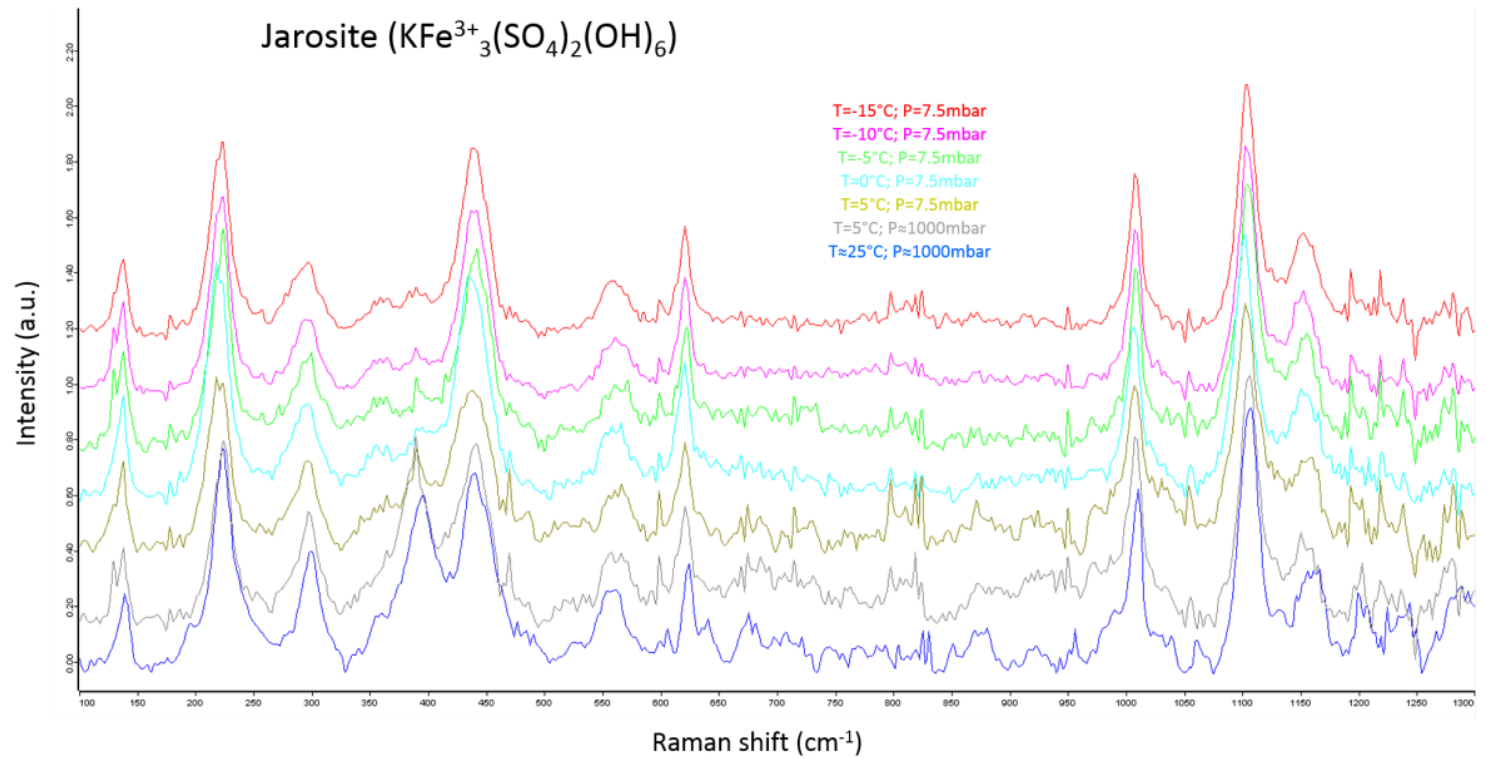

Figure 5.3.a. - Low Raman shift range $\left(100-1300 \mathrm{~cm}^{-1}\right)$ of Jarosite spectra taken at different environmental conditions

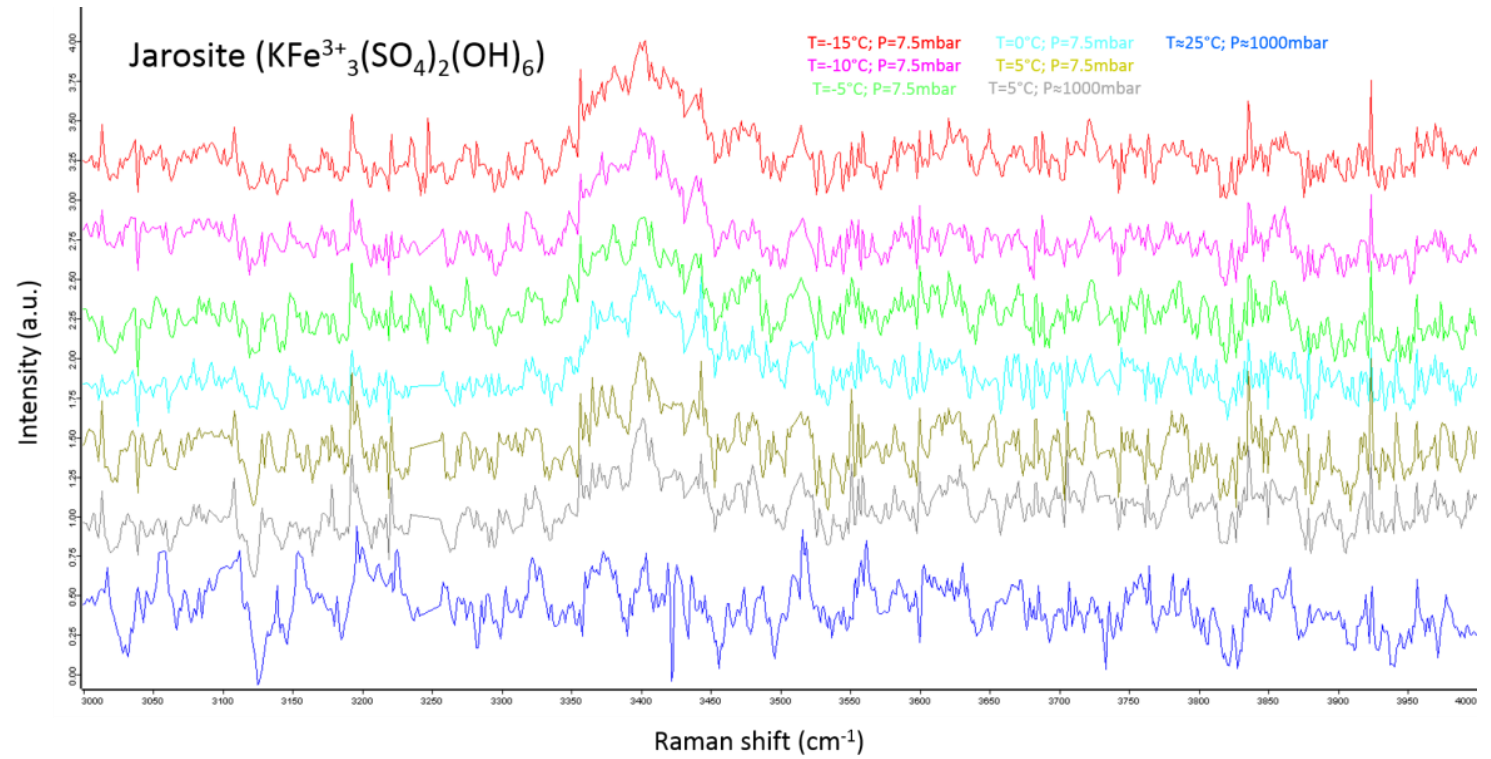

Figure 5.3.b. - High Raman shift range $\left(3000-4000 \mathrm{~cm}^{-1}\right)$ of Jarosite spectra taken at different environmental conditions

Although it was tough to obtain good Jarosite spectra from powder in terms of signal-to-noise ratio (and Figure 5.3.b is the clear proof for this), it can be observed from Figure 5.3.a that there are evident changes occurring. These are 
probably related to changes in the structure that reflect in the appearance of new peaks (see ocre to dark blue spectra in $350-400 \mathrm{~cm}^{-1}$ wavenumber range).

The numbers for these variations are listed in Table 5.3. Variations of even $5 \mathrm{~cm}^{-1}$ and, above all the appearance of new bands, may account for effects beyond peak fitting variations due to the low signal-to-noise spectra.

These effects may be produced by the laser heating and transforming the sample locally despite the precautions taken in selecting the irradiance value for the analysis. 


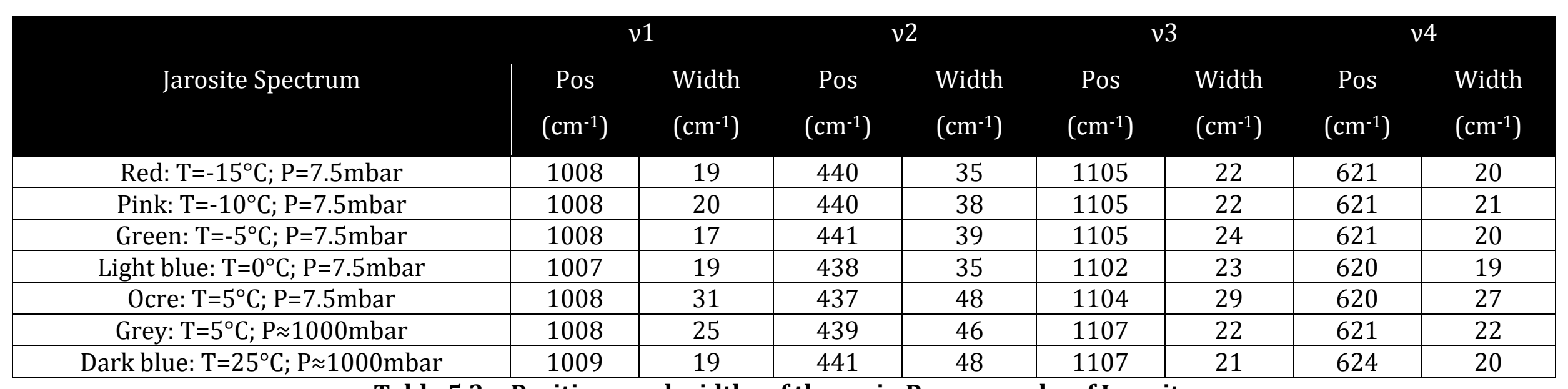

Table 5.3. - Positions and widths of the main Raman peaks of Jarosite 


\subsubsection{Quartz}

The last sample presented here is Quartz. Figure 5.4 shows the spectra taken at different environmental conditions. The high Raman shift region is not presented in this case, as it is not relevant due to the absence of peaks.

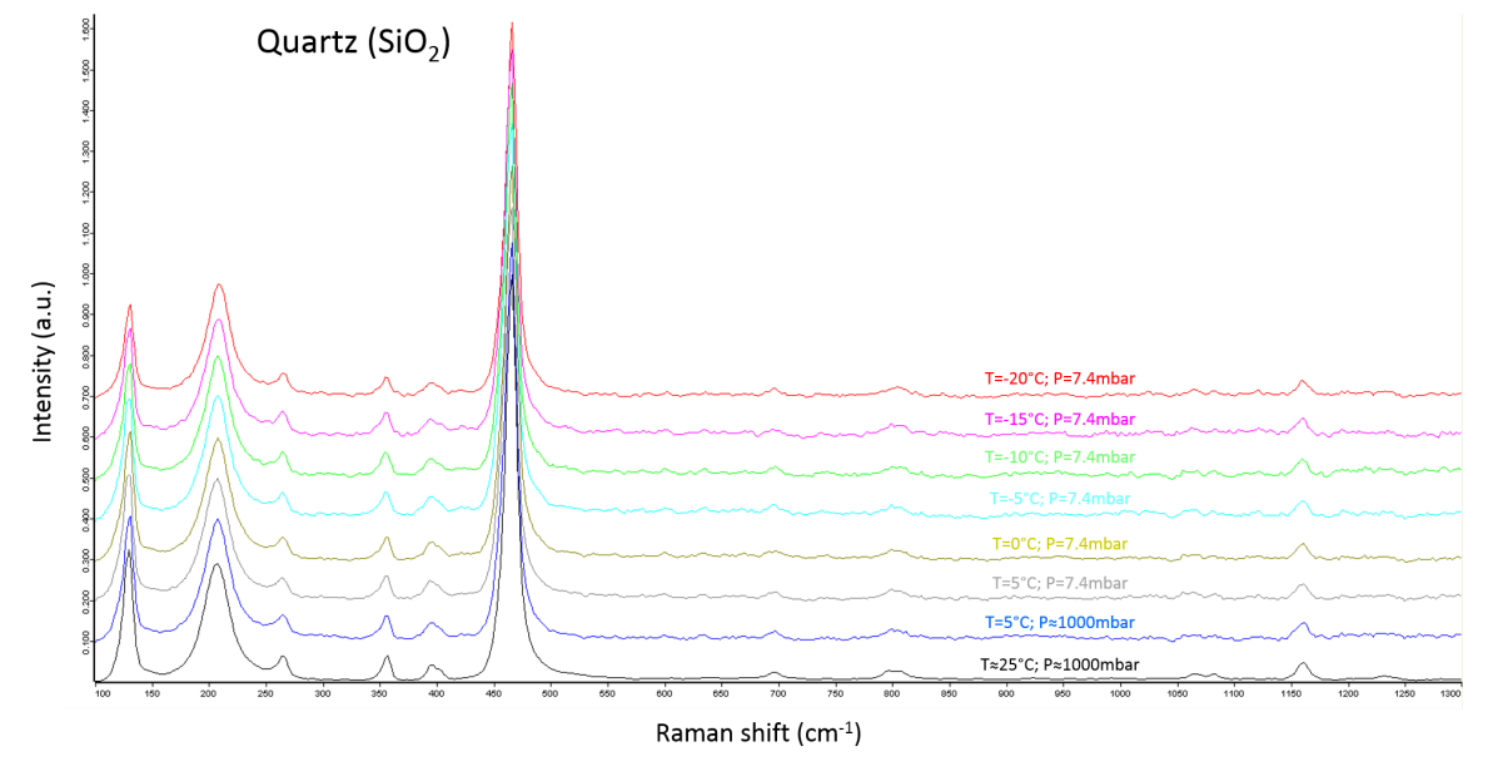

Figure 5.4. - Low Raman shift region $\left(100-1300 \mathrm{~cm}^{-1}\right)$ of Quartz spectra taken at different environmental conditions

As it can be seen from the figure, under the RLS-ExoMars relevant environmental conditions, there are no evident changes in Raman spectra of Quartz that justify its inclusion in a mission-specific spectral database. Anyway, Table 5.4 summarizes the data for the main Raman peaks of Quartz.

This table, once again, presents no relevant changes beyond typical missadjustments in fittings due to noise at the sides of the peaks or due to the tails of adjacent Raman bands. 


\begin{tabular}{|c|c|c|c|c|c|c|}
\hline Quartz Spectrum & $\begin{array}{c}\text { Pos } \\
\left(\mathrm{cm}^{-1}\right)\end{array}$ & $\begin{array}{c}\text { Width } \\
\left(\mathrm{cm}^{-1}\right)\end{array}$ & \multicolumn{2}{c|}{$\begin{array}{c}\text { Pos } \\
\left(\mathrm{cm}^{-1}\right)\end{array}$} & $\begin{array}{c}\text { Width } \\
\left(\mathrm{cm}^{-1}\right)\end{array}$ & $\begin{array}{c}\text { Pos } \\
\left(\mathrm{cm}^{-1}\right)\end{array}$ \\
\hline Red: $\mathrm{T}=-20^{\circ} \mathrm{C} ; \mathrm{P}=7.4 \mathrm{mbar}$ & 466 & 15 & 209 & 39 & 129 & 18 \\
\hline Pink: $\mathrm{T}=-15^{\circ} \mathrm{C} ; \mathrm{P}=7.4 \mathrm{mbar}$ & 465 & 14 & 208 & 34 & 129 & 14 \\
\hline Green: $\mathrm{T}=-10^{\circ} \mathrm{C} ; \mathrm{P}=7.4 \mathrm{mbar}$ & 465 & 14 & 208 & 31 & 129 & 13 \\
\hline Light blue: $\mathrm{T}=-5^{\circ} \mathrm{C} ; \mathrm{P}=7.4 \mathrm{mbar}$ & 465 & 14 & 207 & 32 & 129 & 13 \\
\hline Ocre: $\mathrm{T}=0^{\circ} \mathrm{C} ; \mathrm{P}=7.4 \mathrm{mbar}$ & 465 & 14 & 208 & 32 & 129 & 13 \\
\hline Grey: $\mathrm{T}=5^{\circ} \mathrm{C} ; \mathrm{P}=7.4 \mathrm{mbar}$ & 465 & 14 & 207 & 33 & 129 & 13 \\
\hline Dark blue: $\mathrm{T}=5^{\circ} \mathrm{C} ; \mathrm{P} \approx 1000 \mathrm{mbar}$ & 465 & 13 & 208 & 31 & 129 & 12 \\
\hline Black: $\mathrm{T}=25^{\circ} \mathrm{C} ; \mathrm{P} \approx 1000 \mathrm{mbar}$ & 466 & 13 & 207 & 28 & 129 & 11 \\
\hline
\end{tabular}

Table 5.4. - Positions and widths of the main Raman peaks of Quartz 


\subsection{Experiment 2: Sweep in power}

This second science test seeks to find the minimum irradiance at which the mineral grains of our selected samples get affected by thermal effects derived from exposure to focused laser light.

This experiment was performed in both RLS-ExoMars relevant conditions $\left(\mathrm{T}=-15^{\circ} \mathrm{C}^{2}\right.$ and $\left.\mathrm{p}_{\mathrm{CO} 2} \approx 7.5 \mathrm{mbar}\right)$ and Earth lab conditions $\left(\mathrm{T}=20^{\circ} \mathrm{C}\right.$ and atmospheric pressure in the lab).

\subsubsection{Methodology}

The procedure of this test is divided into two subtests: an engineering one to visually verify the flattening performance of the flattening system, and a scientific one studying the effect of the power sweep on the selected thermolabile samples. This procedure can be expressed by this pseudocode:

Placement of the powder sample on the RC inside the RLS-ExoMars Simulation Chamber. Closing of the chamber.

Establishment of the RLS-ExoMars relevant conditions.

Flattening of the sample,

for lines from 1 to 3

for points along the line from 1 to 20 spaced $500 \mu \mathrm{m}$ Measurement of the height of the sample with respect to the surface of the RC.

end

end

for mineral grains from 1 to 5

Selection of a mineral grain to analyse;

Focus.

Picture before at the focus position.

for input power values from $5 \%$ until damage in steps of $5 \%$

5 -min (as a maximum) Raman spectrum acquisition.

Picture after acquisition. // To see if there has been damage to the grain

2 PPL-ENG-ALD-0180 in (Ball et al., 2013): Temperature of the samples on the RC during $\mu$ Omega and RLS operations $<-5^{\circ} \mathrm{C}$

3 PPL-ENG-LAB-0090 in (Ball et al., 2013): $50 \%$ of the grains in the RC within 100 $\mu \mathrm{m}$ from the surface, $80 \%$ within $200 \mu \mathrm{m}$ 
end

end

Go to Earth lab conditions. // These are extra measurements to show differences

under other environmental conditions

for mineral grains from 1 to 5

Selection of a mineral grain to analyse.

Focus.

Picture before at the focus position.

for input power values from 5\% until damage in steps of $5 \%$ 5-min (as a maximum) Raman spectrum acquisition.

Picture after acquisition. // To see if there has been damage to the

end

end

The obtained spectra have been calibrated in wavelength following the code in Annex C and the Hg-Ar and Ne lamps. Cyclohexane has been used as a standard to check this calibration. Due to the low intensity of the spectra, intensity calibration showed so much noise at the edges of the spectrum due to the compensation for the low efficiency of the system there, that masked the heating effects wanted to be shown. Therefore, and exceptionally, it is not applied here.

\subsubsection{Samples}

Two samples were selected to be deeply studied under these two experiments: jarosite and hematite.

Jarosite has already been presented in the previous section.

Hematite is the mineral form of an iron oxide $\left(\mathrm{Fe}_{2} \mathrm{O}_{3}\right)$. On Earth this mineral is generally found as a precipitate of springs and standing masses of water. Its presence has been detected on Mars from orbit (Christensen et al., 2000; Bibring et al., 2006) and in situ (Squyres et al., 2004).

For these experiments a quantity of these samples enough to fill the Refillable Container (RC) was prepared so that $40 \%$ in mass of the mineral grains corresponded to the $50-125 \mu \mathrm{m}$ grain size distribution region, and $60 \%$ in mass to the $125-250 \mu \mathrm{m}$ range. The lower range $(50-250 \mu \mathrm{m})$ of the grain size distribution 
planned for ExoMars (Ball, 2013) was here selected, as it is the most critical fraction regarding this thermolability study in terms of heat dissipation.

\subsubsection{Jarosite}

The first sample to be analysed was jarosite in the previously specified grain size distribution.

\subsection{Engineering data}

Before showing the scientific products of this analysis, the pressure and temperature curves recorded during the process as well as the 3 line profiles are shown in the next two sub-sections.

\subsection{Pressure and Temperature curves}

As the pressure and temperature values remain stable after reaching the desired values, only the adaptation period of time is shown in Figure 5.5.

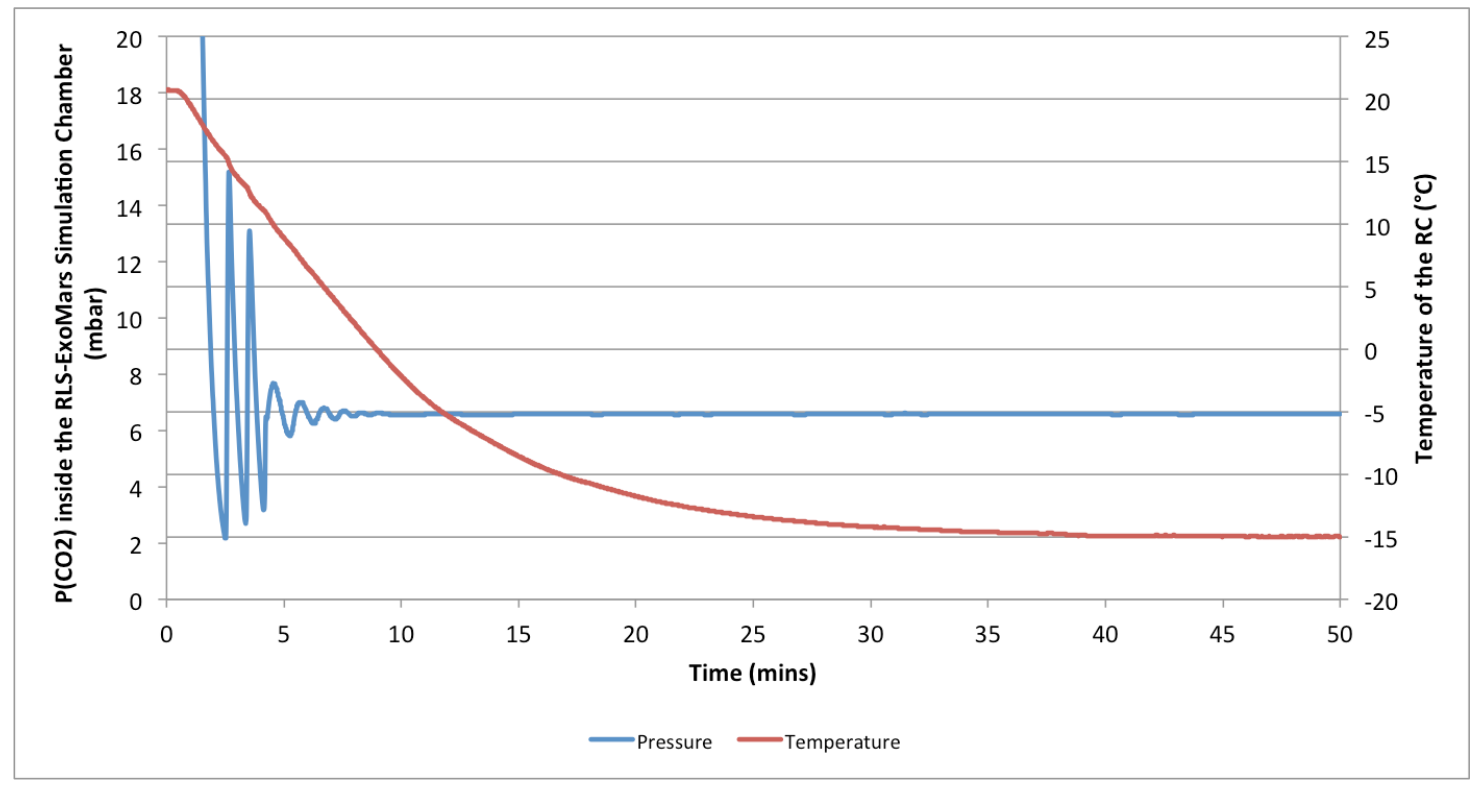

Figure 5.5. - Pressure and temperature curves for the period of achievement of the RLS-ExoMars relevant conditions. The pressure axis has been cropped to show the 'important' region.

The oscillation that the pressure curve shows is produced by the internal PID system of the pressure controller acting on the electro-actuated gas inlet valve.

As it can be seen, the temperature and pressure conditions are stable in less than one hour. 


\subsection{Flattening performance}

Figure 5.6 presents two images corresponding to jarosite before and after being flattened under RLS-ExoMars relevant conditions.

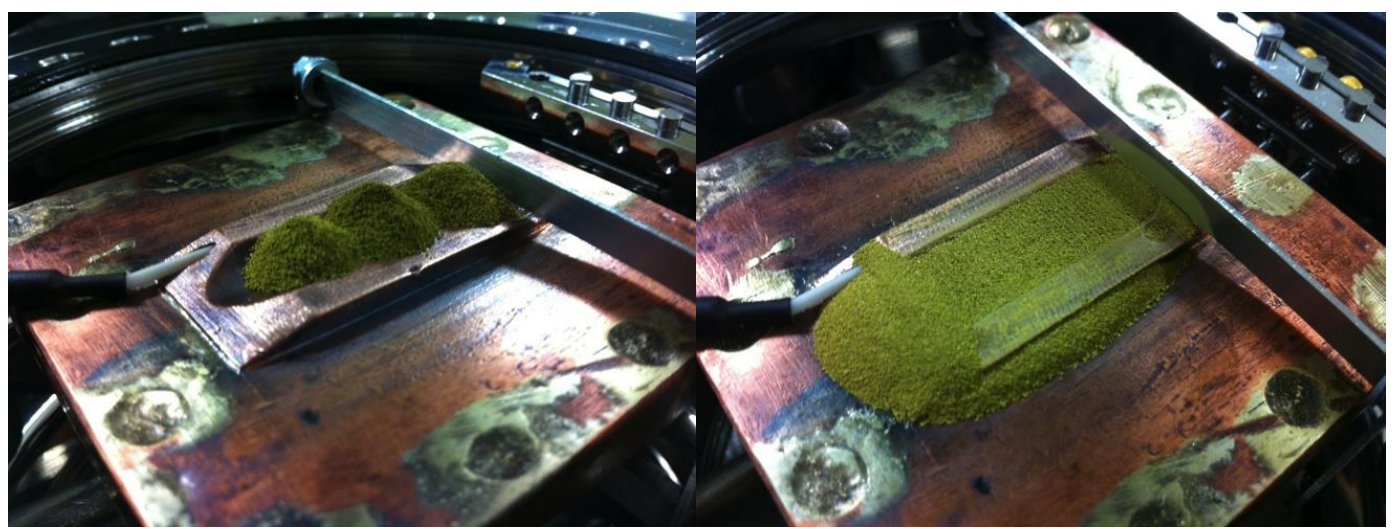

Figure 5.6. - Jarosite before (left) and after (right) flattening under RLSExoMars relevant conditions.

Figure 5.7 shows the vertical profile for three central lines of the RC.

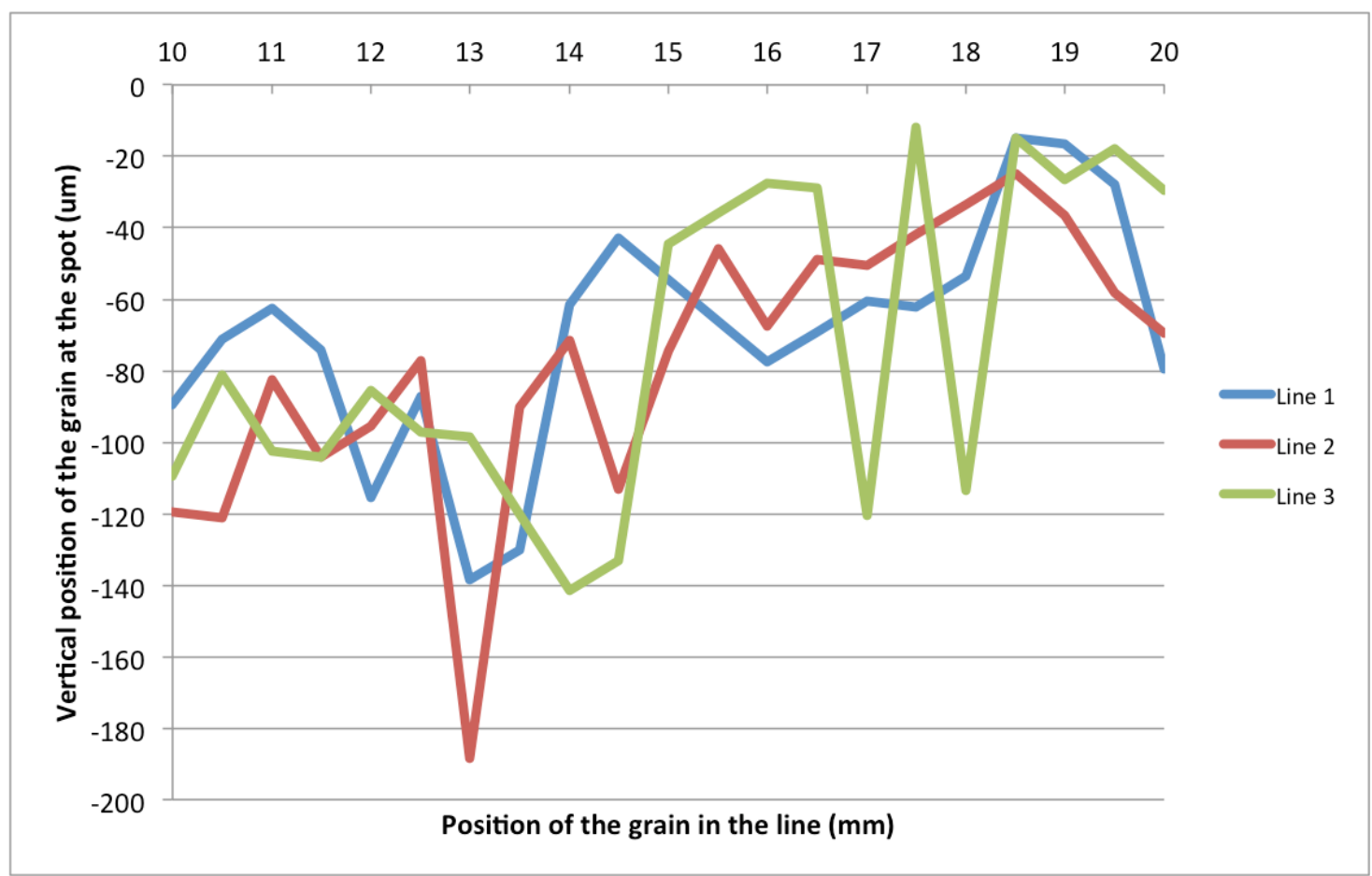

Figure 5.7. - Vertical profile of three lines in the central region of the RC

Table 5.5 shows the statistic values for those three lines. It can be seen that all three lines comply with the requirement ${ }^{3}$. We can thus say that the flattening performance for jarosite is satisfactory. 


\begin{tabular}{|c|c|c|c|}
\hline & Line 1 & Line 2 & Line 3 \\
\hline$<100$ & $86 \%$ & $76 \%$ & $62 \%$ \\
\hline$<200$ & $100 \%$ & $100 \%$ & $100 \%$ \\
\hline Mean height $(\mu \mathrm{m})$ & -69 & -77 & -74 \\
\hline Desvest ( $\mu \mathrm{m})$ & 32 & 38 & 44 \\
\hline
\end{tabular}

Table 5.5. - Percentage of points within $100 \mu \mathrm{m}$ and $200 \mu \mathrm{m}$ as well as mean height and standard deviation for the grains in the three profiled lines

\subsection{Results and Discussion}

Table 5.6 to Table 5.10 show the results for jarosite analysed under RLSExoMars relevant conditions. They present the collection of spectra acquired for different irradiances, and the images taken after each spectrum, ordered in increasing irradiance. 


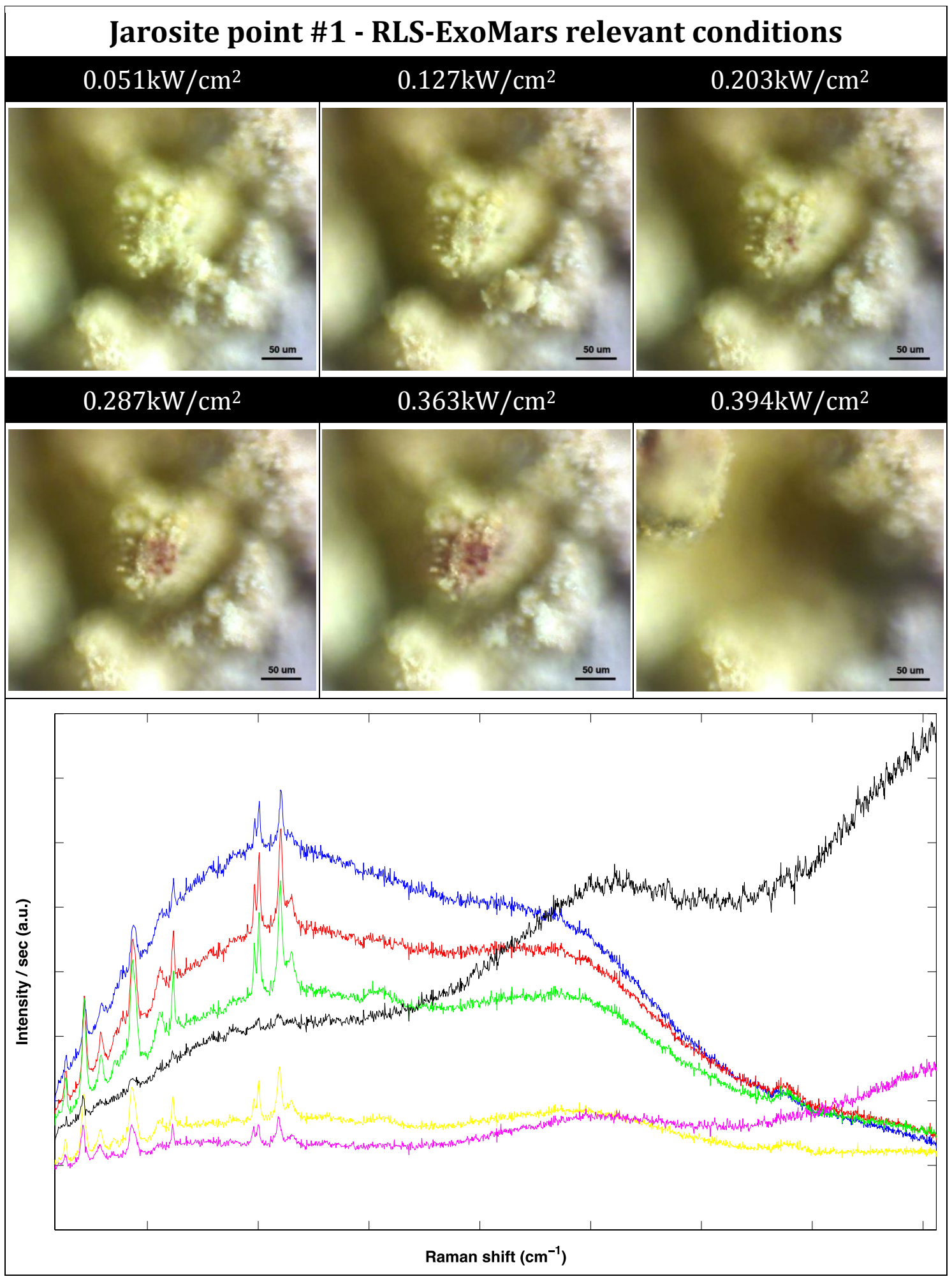

Table 5.6. - Irradiance values, images after exposure to laser and corresponding Raman spectra for the selected point \#1. Blue: $0.051 \mathrm{~kW} / \mathrm{cm}^{2}$;

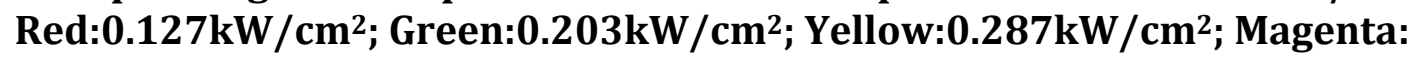
$0.363 \mathrm{~kW} / \mathrm{cm}^{2}$; Black:0.394kW/ $\mathrm{cm}^{2}$. 


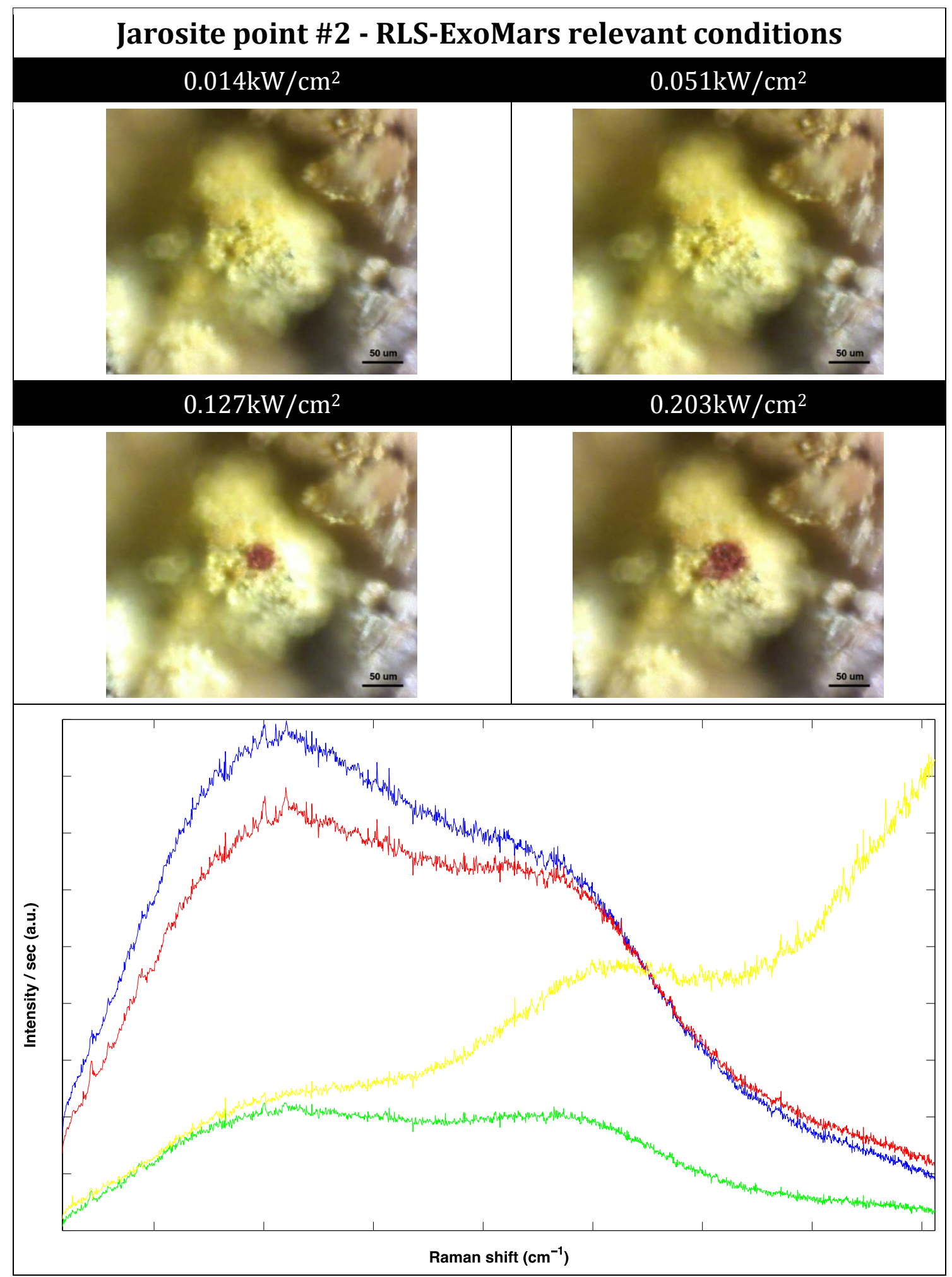

Table 5.7. - Irradiance values, images after exposure to laser and corresponding Raman spectra for the selected point \#2. Blue:0.014kW/ $\mathrm{cm}^{2}$; Red:0.051kW/ $\mathrm{cm}^{2}$; Green:0.127kW/ $\mathrm{cm}^{2}$; Yellow:0.203kW/ $\mathrm{cm}^{2}$. 


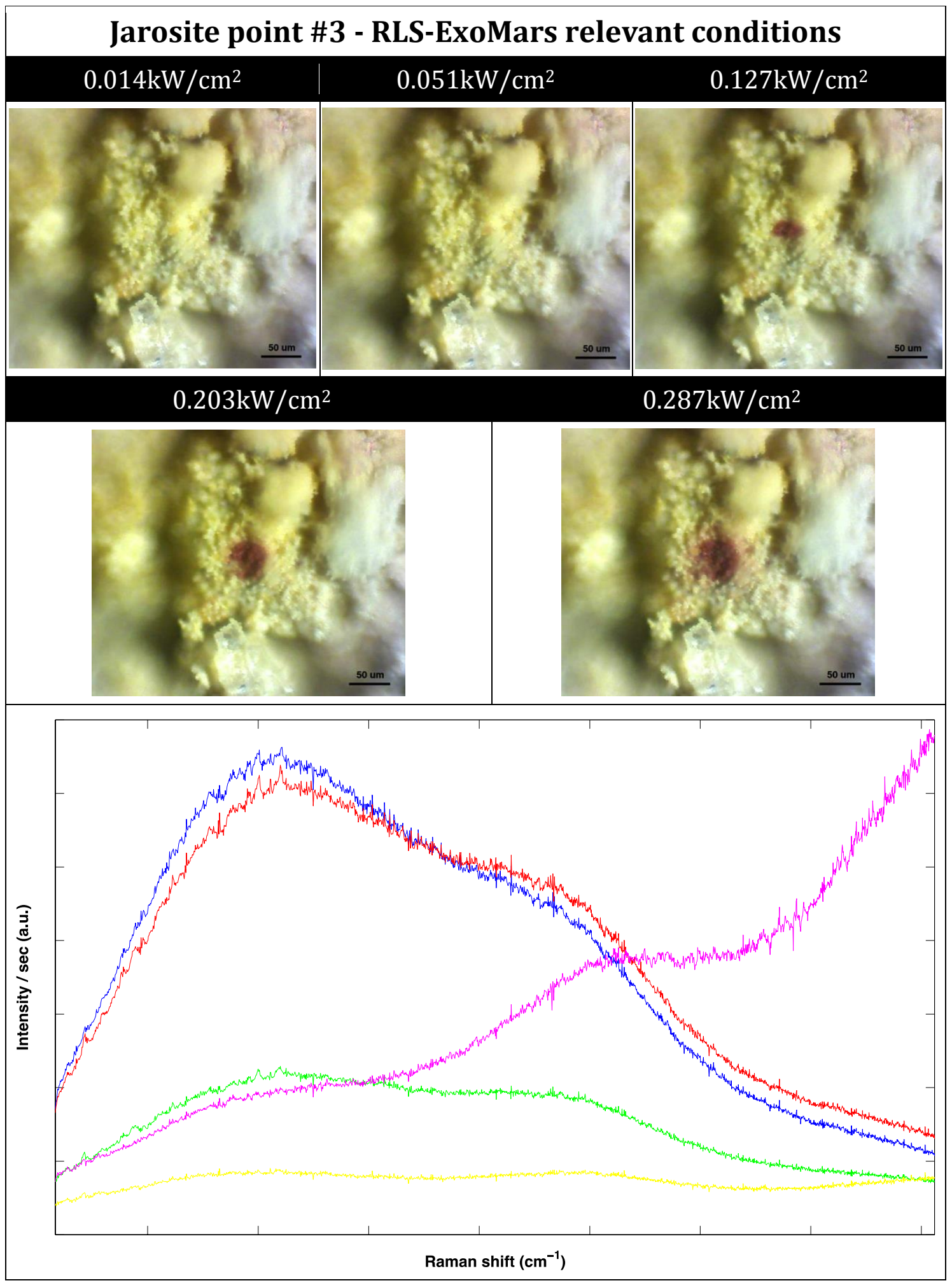

Table 5.8. - Irradiance values, images after exposure to laser and corresponding Raman spectra for the selected point \#2. Blue:0.014kW/ $\mathrm{cm}^{2}$; Red:0.051kW $/ \mathrm{cm}^{2}$; Green:0.127kW $/ \mathrm{cm}^{2}$; Yellow:0.203kW $/ \mathrm{cm}^{2}$; Magenta:0.287kW/ $\mathrm{cm}^{2}$. 


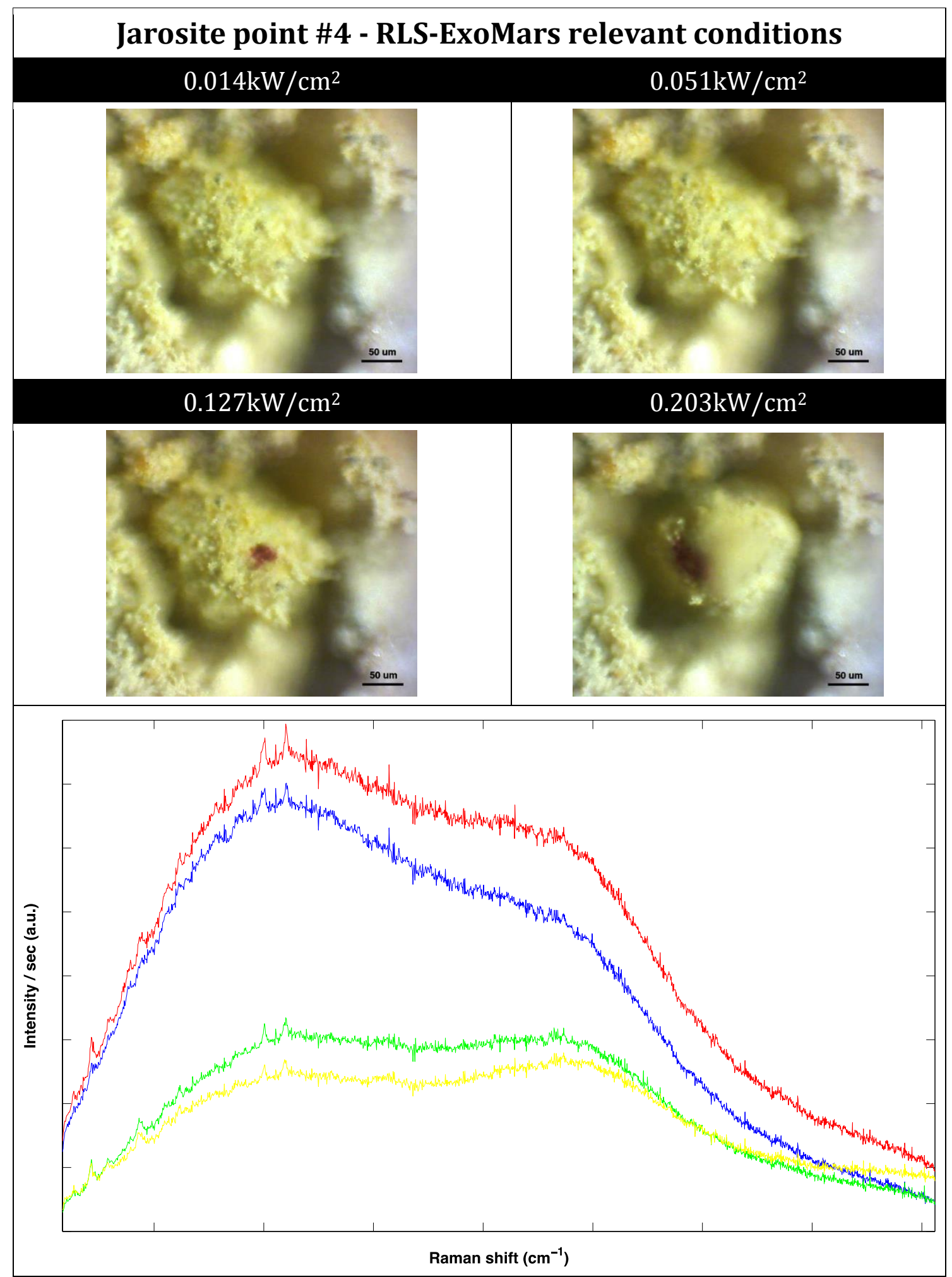

Table 5.9. - Irradiance values, images after exposure to laser and corresponding Raman spectra for the selected point \#4. Blue:0.014kW/ $\mathrm{cm}^{2}$;

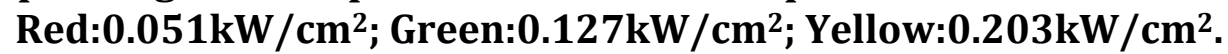




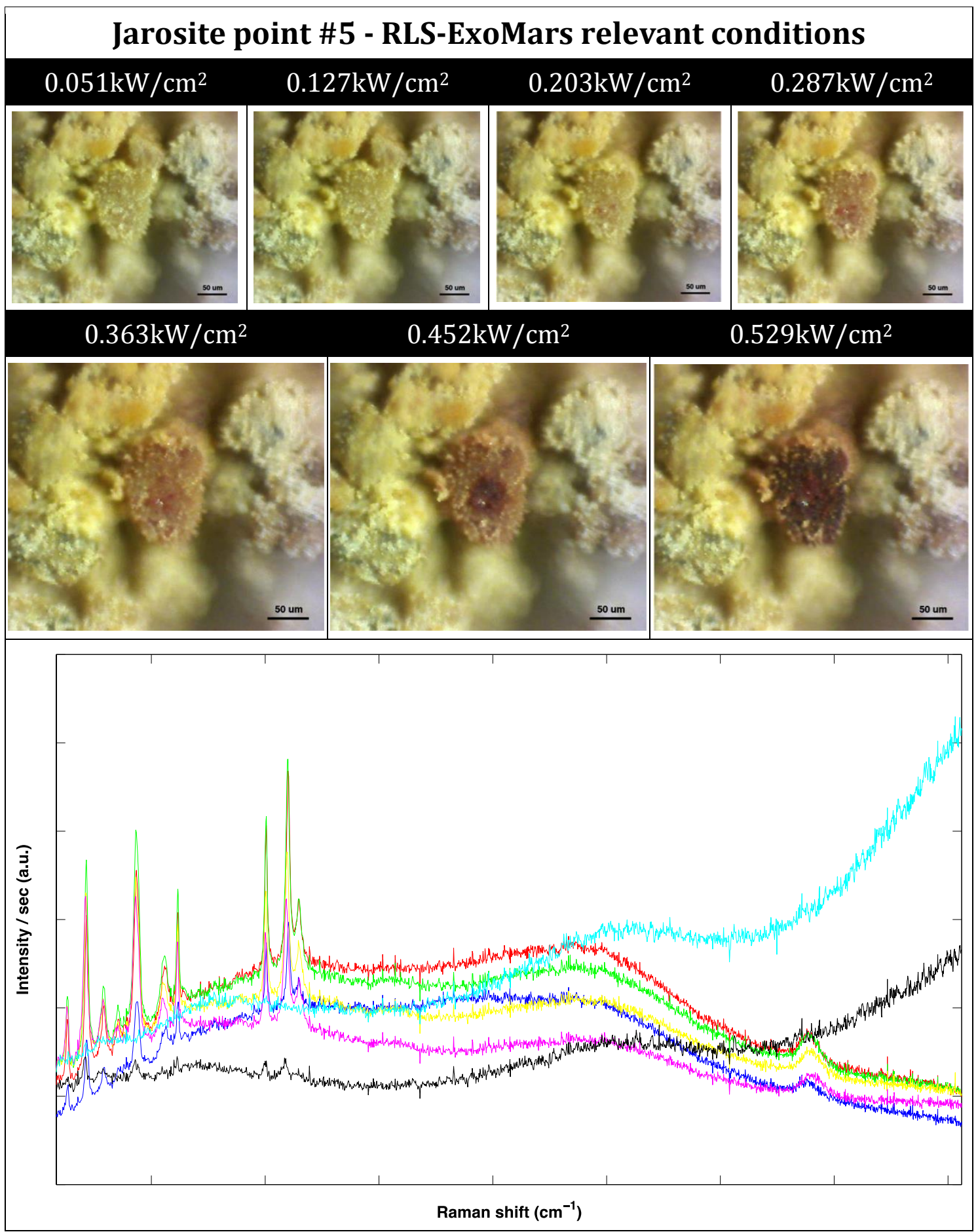

Table 5.10. - Irradiance values, images after exposure to laser and corresponding Raman spectra for the selected point \#1. Blue: $0.051 \mathrm{~kW} / \mathrm{cm}^{2}$;

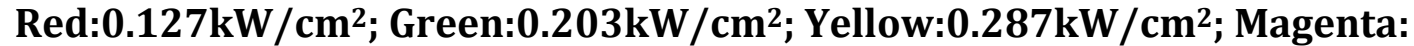
$0.363 \mathrm{~kW} / \mathrm{cm}^{2}$; Black:0.452kW/ $\mathrm{cm}^{2} ;$ Cyan:0.529kW/ $\mathrm{cm}^{2}$. 
In general, the irradiance above which the mineral grain gets visually engraved with the laser is $0.127 \mathrm{~kW} / \mathrm{cm}^{2}$, around $22 \%$ of the minimum RLS specified irradiance $\left(0.6 \mathrm{~kW} / \mathrm{cm}^{2}\right)$. Spectroscopically speaking, Table 5.11 summarizes the results that previous tables present. In it, SNR values for the $\mathbf{v} 1$ mode of jarosite (at $1000 \mathrm{~cm}^{-1}$ ) for the different acquired spectra at different irradiances are listed.

For every spectrum, the SNR is here calculated as the ratio between the intensity of the selected band, measured from the baseline of the spectrum, and the standard deviation of the noise from the peak-free $200 \mathrm{~cm}^{-1}$ baseline-subtracted $1200-1400 \mathrm{~cm}^{-1}$ region. This value is not calculated as specified in the requirements (noise extracted from the subtraction of two consecutive spectra) as, in general, two consecutive spectra are not equivalent due to thermal effects on the grain being analysed. However, this value serves as a good indicator for spectral quality comparison in the frame of the spectra here presented. 


\begin{tabular}{|c|c|c|c|}
\hline Point (\#) & $\begin{array}{l}\text { Laser output } \\
\text { power }(\mathrm{mW})\end{array}$ & $\begin{array}{l}\text { Irradiance } \\
\left(\mathrm{kW} / \mathrm{cm}^{2}\right)\end{array}$ & $\operatorname{SNR}(\mathbf{v} 1)$ \\
\hline \multirow{6}{*}{1} & 1.14 & 0.051 & 14.6 \\
\hline & 2.84 & 0.127 & 27.7 \\
\hline & 4.55 & 0.203 & 34.6 \\
\hline & 6.42 & 0.287 & 20.7 \\
\hline & 8.12 & 0.363 & 11.6 \\
\hline & 10.1 & 0.394 & 4.0 \\
\hline \multirow{4}{*}{2} & 0.32 & 0.014 & 4.5 \\
\hline & 1.14 & 0.051 & 7.1 \\
\hline & 2.84 & 0.127 & 5.0 \\
\hline & 4.55 & 0.203 & 3.7 \\
\hline \multirow{5}{*}{3} & 0.32 & 0.014 & 4.2 \\
\hline & 1.14 & 0.051 & 6.5 \\
\hline & 2.84 & 0.127 & 5.9 \\
\hline & 4.55 & 0.203 & 3.0 \\
\hline & 6.42 & 0.287 & 2.4 \\
\hline \multirow{4}{*}{4} & 0.32 & 0.014 & 5.4 \\
\hline & 1.14 & 0.051 & 6.1 \\
\hline & 2.84 & 0.127 & 8.1 \\
\hline & 4.55 & 0.203 & 5.5 \\
\hline \multirow{7}{*}{5} & 1.14 & 0.051 & 27.0 \\
\hline & 2.84 & 0.127 & 48.1 \\
\hline & 4.55 & 0.203 & 53.9 \\
\hline & 6.42 & 0.287 & 38.7 \\
\hline & 8.12 & 0.363 & 36.2 \\
\hline & 10.1 & 0.452 & 6.6 \\
\hline & 11.8 & 0.529 & 3.1 \\
\hline
\end{tabular}

Table 5.11. - SNR values for the v1 peak of jarosite in all the RLS-ExoMarslike previous spectra, and their corresponding irradiance values and associated powers. Cells highlighted in green show best SNR values for each point.

The best spectra, those with the highest Raman SNRs, are achieved for lower or higher irradiances compared to the visual, depending on whether the mineral grain is a conglomerate of smaller grains or a well-crystallized grain of jarosite. Mineral grains \#1 and \#5 are, visually, the best crystallized of the whole selection. SNR values for them are higher in comparison to the rest of the grains and they withstand higher irradiances before their spectra start to degrade.

The common behaviour for spectral degradation is to get, from the spectrum that we can consider the best, progressively lower quality spectra as irradiance increases, continuing with the growth of a characteristic spectral feature: a 'spectral tail' appearing at high Raman shift (RS). This feature is 
associated with a black-body radiation signature emitted by the material being analyzed and centered in the mid-infrared region (Bowie et al., 2000). At this point the Raman peaks become very poor.

Table 5.12 to Table 5.21 show the results for the analyses done to jarosite mineral grains under Earth lab conditions $\left(\mathrm{T}=20^{\circ} \mathrm{C}\right.$ and Earth atmospheric pressure). As, under these environmental conditions, the mineral grains put up with higher values of irradiance, each point has been divided into two tables to show both, the low and high range of irradiance values. 


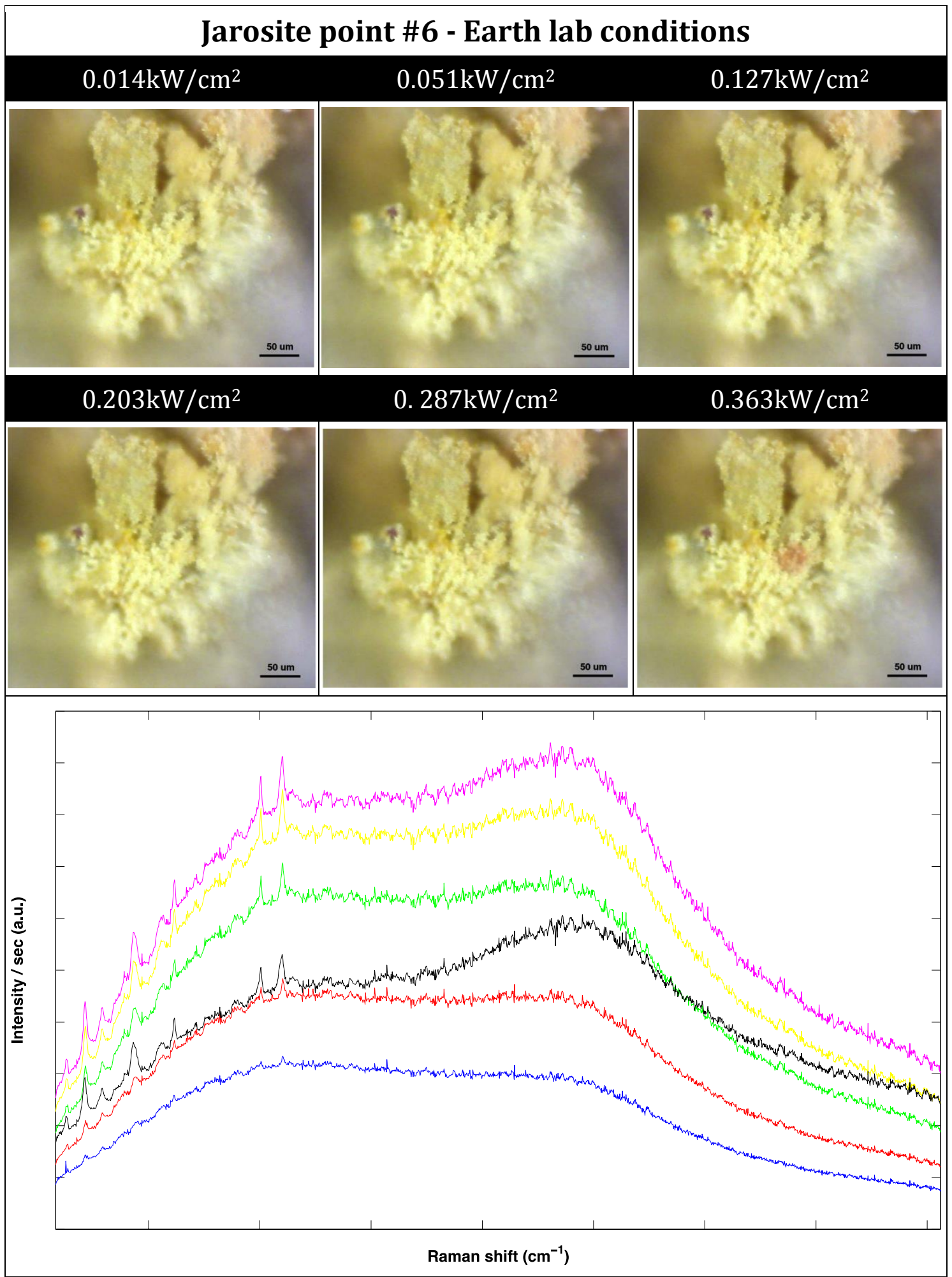

Table 5.12. - Irradiance values, images after exposure to laser and corresponding Raman spectra for the selected point \#6. Blue:0.014kW/ $\mathrm{cm}^{2}$;

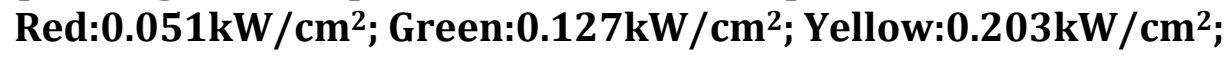
Magenta:0.287kW/ $\mathrm{cm}^{2}$; Black: $0.363 \mathrm{~kW} / \mathrm{cm}^{2}$. 


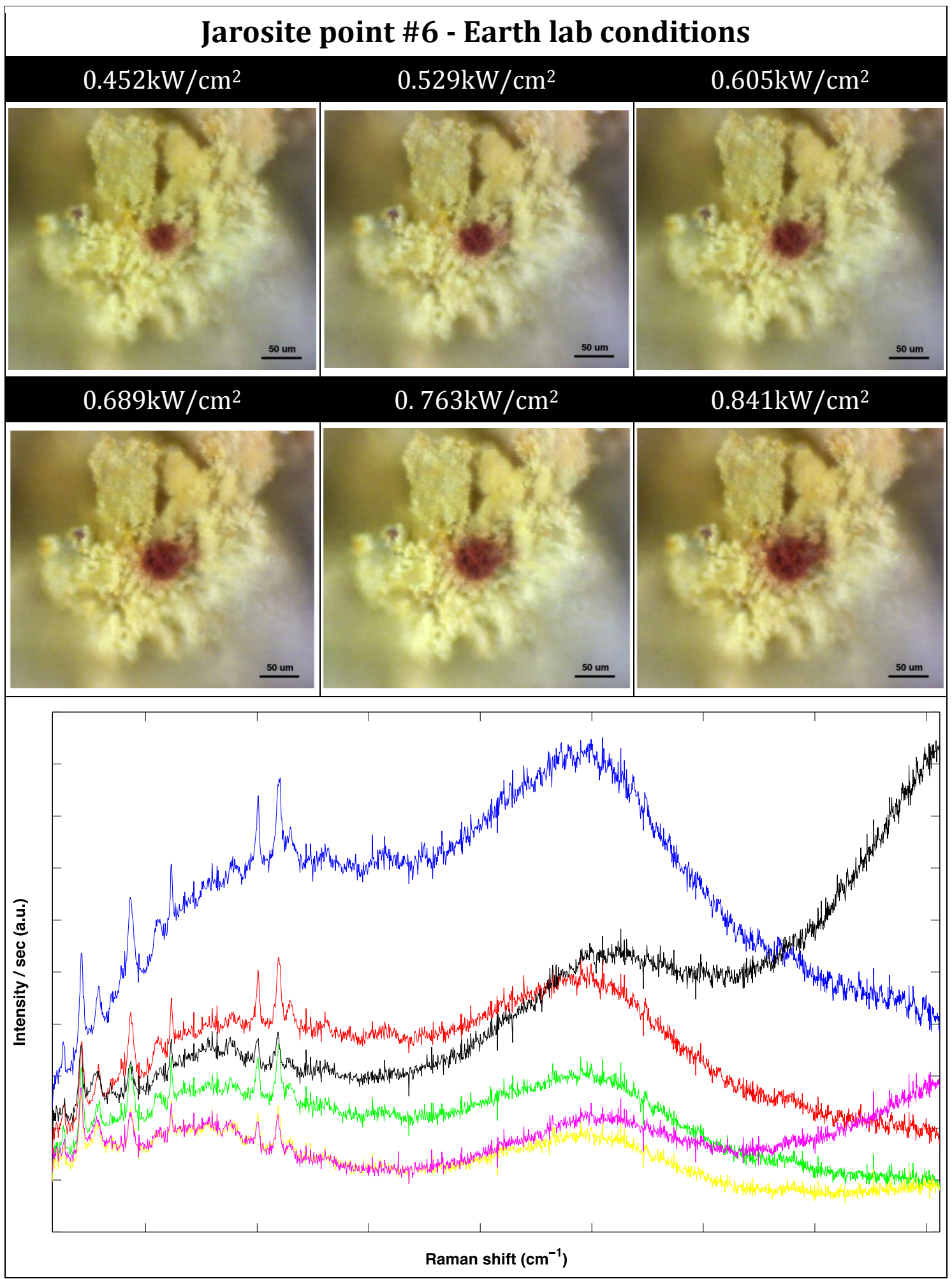

Table 5.13. - Irradiance values, images after exposure to laser and corresponding Raman spectra for the selected point \#6. Blue: $0.452 \mathrm{~kW} / \mathrm{cm}^{2}$;

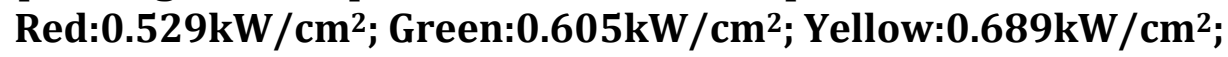
Magenta:0.763kW/ $\mathrm{cm}^{2}$; Black: $0.841 \mathrm{~kW} / \mathrm{cm}^{2}$. 


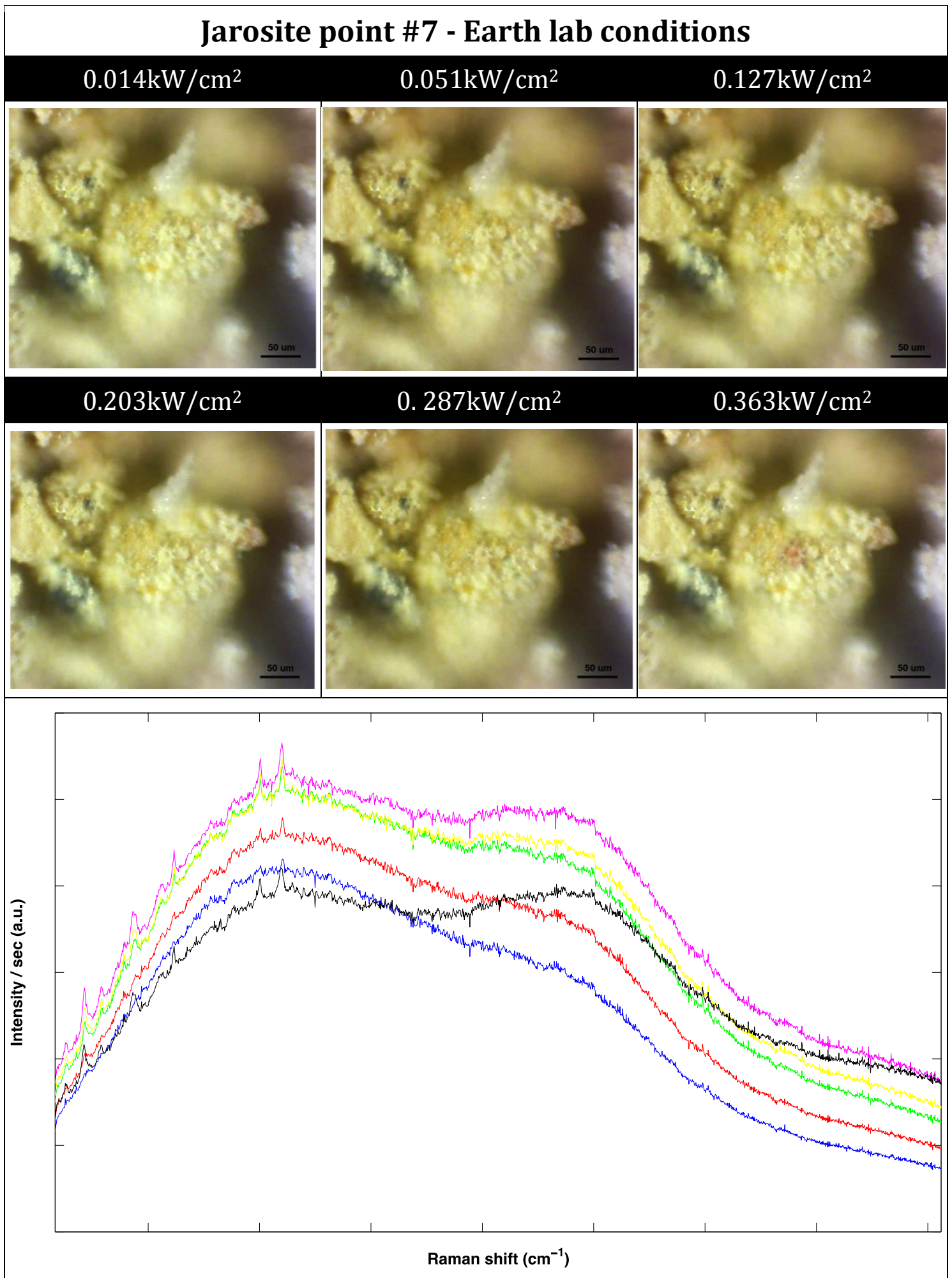

Table 5.14. - Irradiance values, images after exposure to laser and corresponding Raman spectra for the selected point \#7. Blue:0.014kW/ $\mathrm{cm}^{2}$; Red:0.051kW/ $\mathrm{cm}^{2}$; Green:0.127kW/ $\mathrm{cm}^{2}$; Yellow:0.203kW/ $\mathrm{cm}^{2}$; Magenta:0.287kW $/ \mathrm{cm}^{2}$; Black: $0.363 \mathrm{~kW} / \mathrm{cm}^{2}$. 


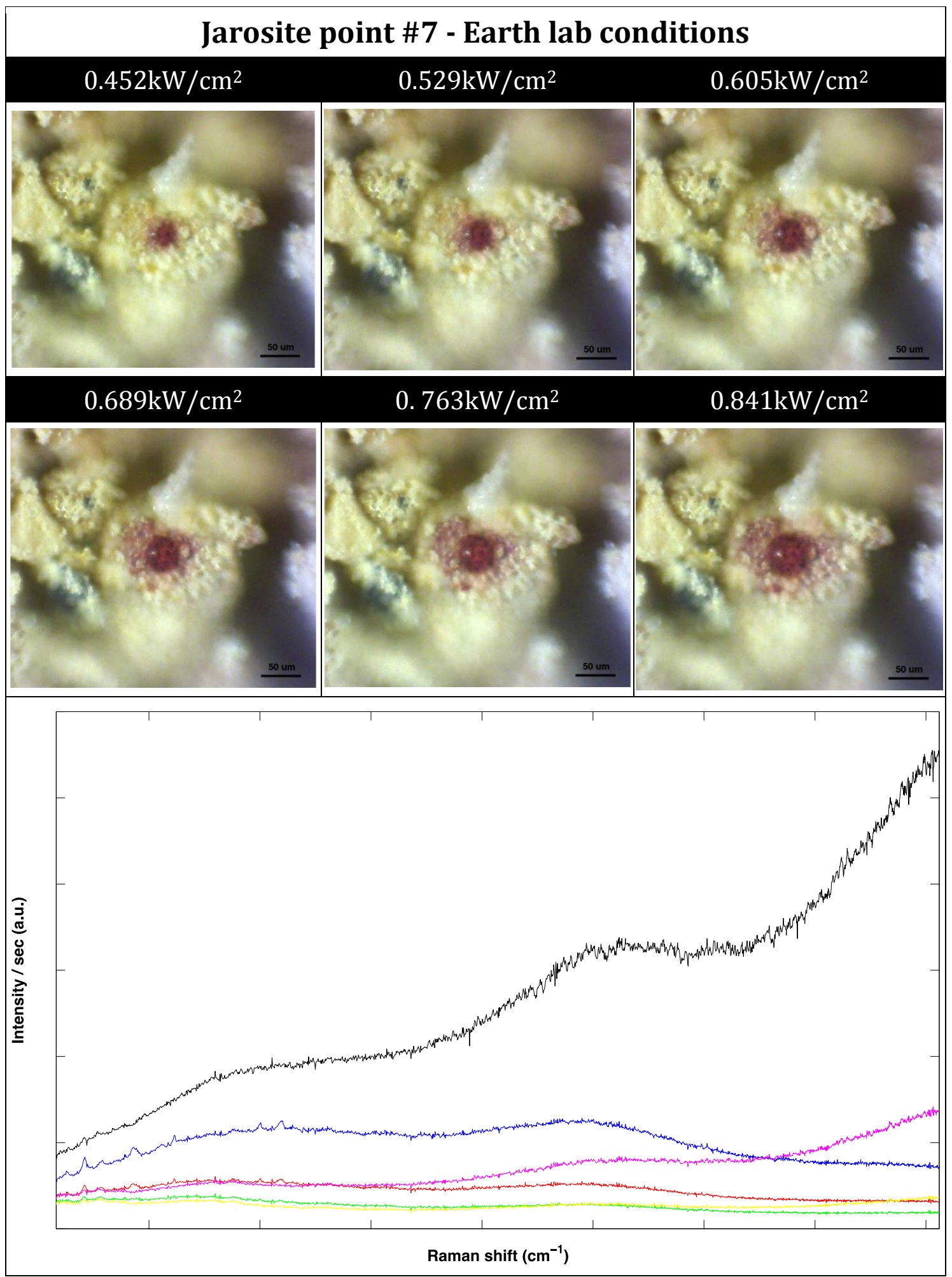

Table 5.15. - Irradiance values, images after exposure to laser and corresponding Raman spectra for the selected point \#7. Blue:0.452kW/ $\mathrm{cm}^{2}$; Red:0.529kW/ $\mathrm{cm}^{2}$; Green:0.605kW $/ \mathrm{cm}^{2}$; Yellow:0.689kW $/ \mathrm{cm}^{2}$; Magenta:0.763kW $/ \mathrm{cm}^{2}$; Black: $0.841 \mathrm{~kW} / \mathrm{cm}^{2}$. 


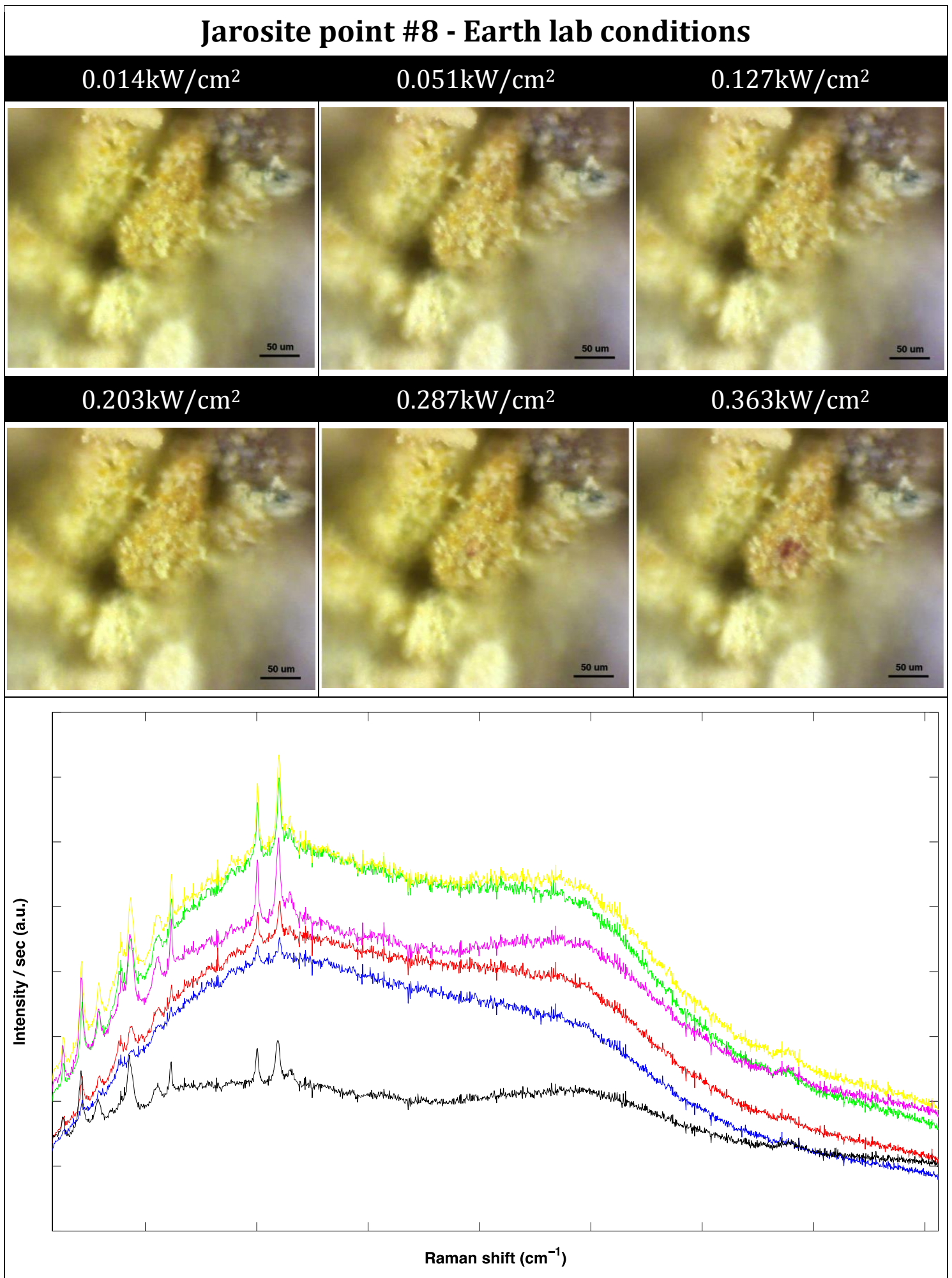

Table 5.16. - Irradiance values, images after exposure to laser and corresponding Raman spectra for the selected point \#8. Blue:0.014kW $/ \mathrm{cm}^{2}$; Red:0.051kW/ $\mathrm{cm}^{2}$; Green:0.127kW/ $\mathrm{cm}^{2}$; Yellow:0.203kW/ $\mathrm{cm}^{2}$; Magenta:0.287kW/ $\mathrm{cm}^{2}$; Black: $0.363 \mathrm{~kW} / \mathrm{cm}^{2}$. 


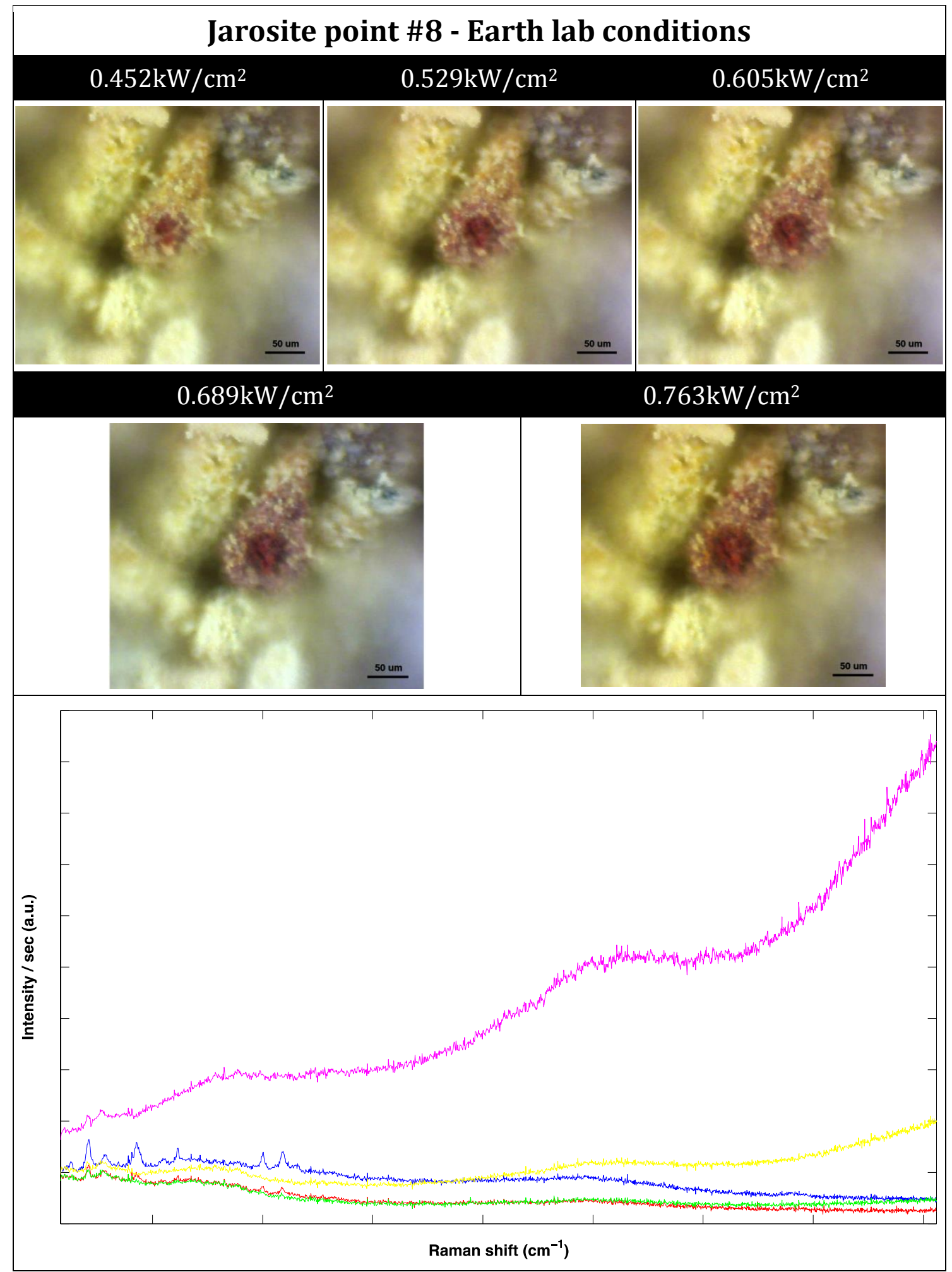

Table 5.17. - Irradiance values, images after exposure to laser and corresponding Raman spectra for the selected point \#8. Blue: $0.452 \mathrm{~kW} / \mathrm{cm}^{2}$;

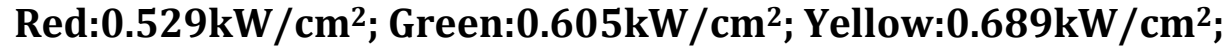
Magenta:0.763kW/ $\mathrm{cm}^{2}$. 


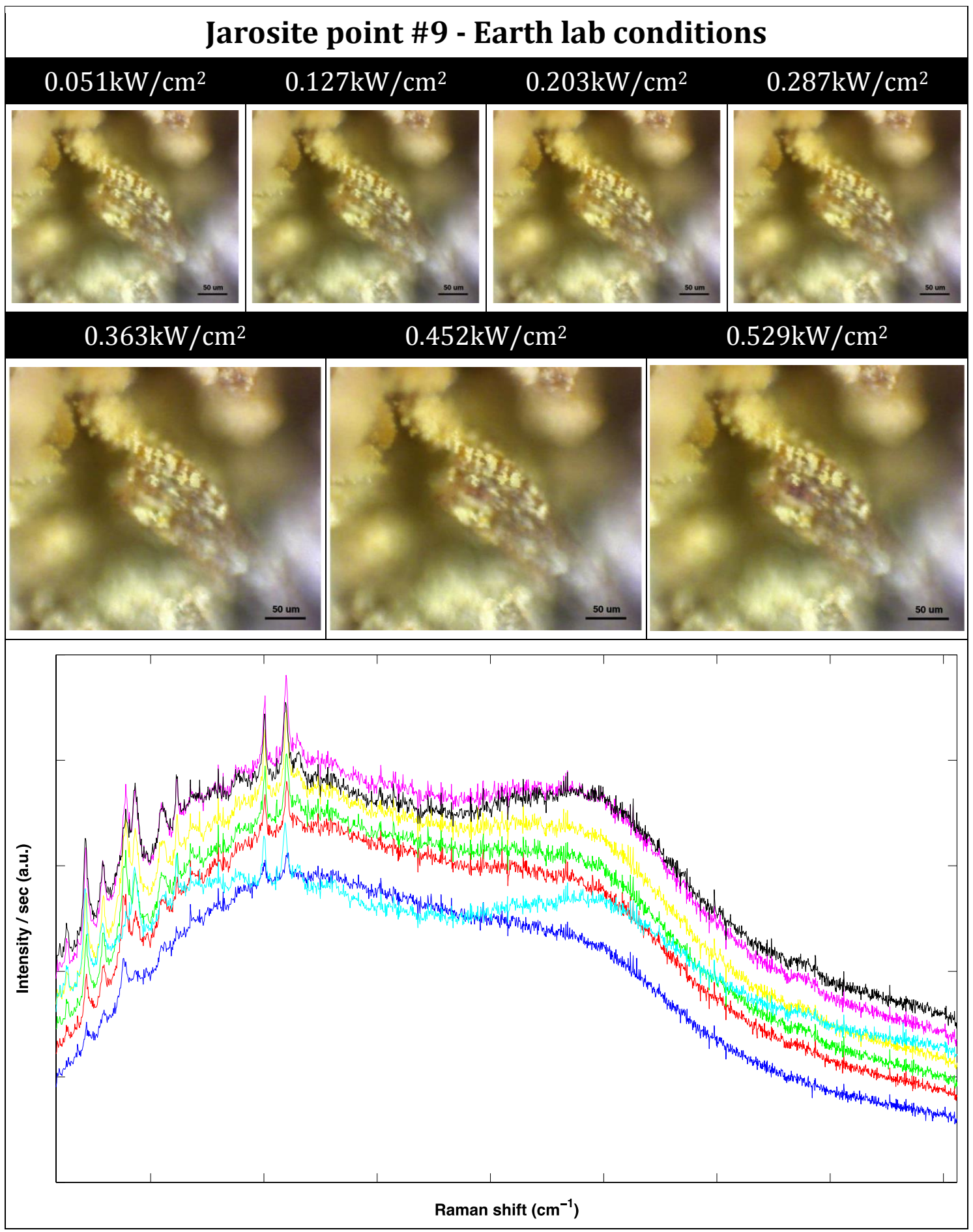

Table 5.18. - Irradiance values, images after exposure to laser and corresponding Raman spectra for the selected point \#9. Blue: $0.051 \mathrm{~kW} / \mathrm{cm}^{2}$;

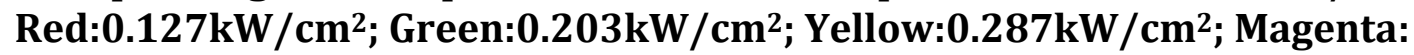

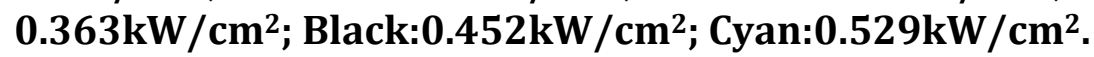




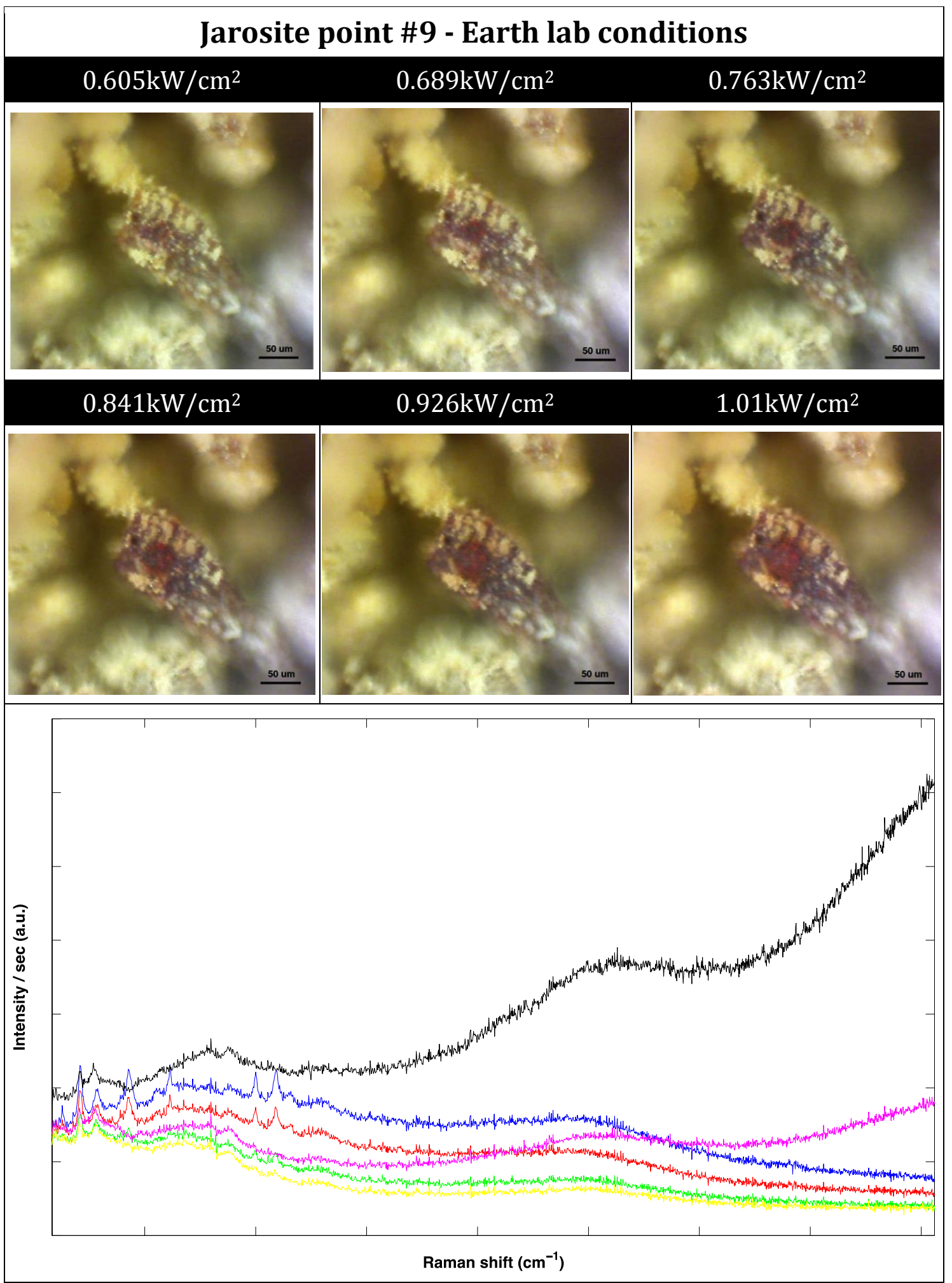

Table 5.19. - Irradiance values, images after exposure to laser and corresponding Raman spectra for the selected point \#9. Blue:0.605kW/ $/ \mathrm{cm}^{2}$; Red:0.689kW $/ \mathrm{cm}^{2}$; Green:0.763kW $/ \mathrm{cm}^{2}$; Yellow:0.841kW $/ \mathrm{cm}^{2}$; Magenta: $0.926 \mathrm{~kW} / \mathrm{cm}^{2}$; Black:1.01kW/ $\mathrm{cm}^{2}$. 


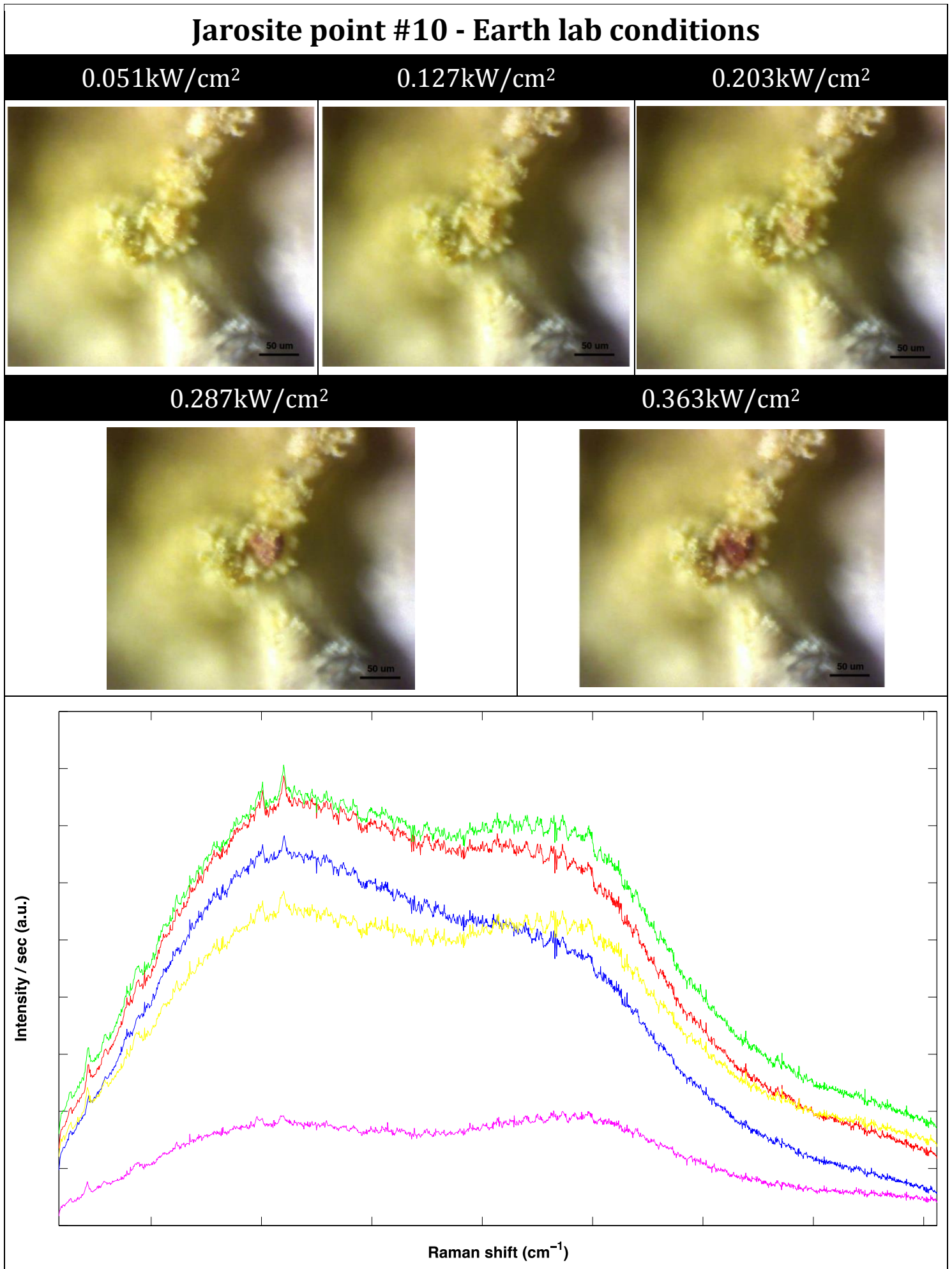

Table 5.20. - Irradiance values, images after exposure to laser and corresponding Raman spectra for the selected point \#10. Blue:0.051kW/ $\mathrm{cm}^{2}$; Red:0.127kW/ $\mathrm{cm}^{2}$; Green:0.203kW/ $\mathrm{cm}^{2}$; Yellow:0.287kW $/ \mathrm{cm}^{2}$; Magenta: $0.363 \mathrm{~kW} / \mathrm{cm}^{2}$. 


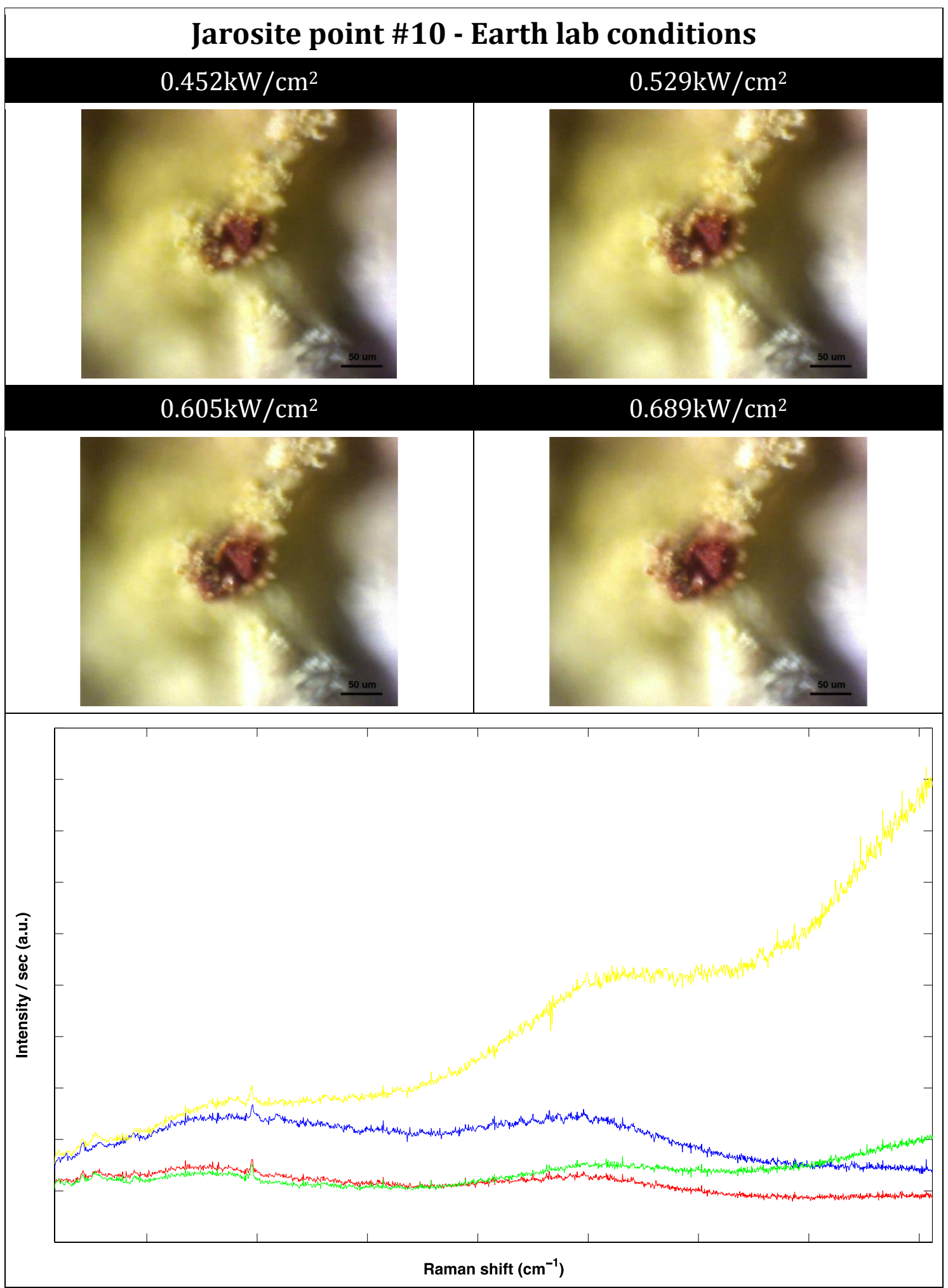

Table 5.21. - Irradiance values, images after exposure to laser and corresponding Raman spectra for the selected point \#10. Blue:0.452kW/ $\mathrm{cm}^{2}$; Red:0.529kW/cm²; Green:0.605kW/ $\mathrm{cm}^{2}$; Yellow:0.689kW/ $\mathrm{cm}^{2}$. 
In this case, mineral grains appear to get damaged visually generally below $0.363 \mathrm{~kW} / \mathrm{cm}^{2}$, which is a higher value compared to RLS-ExoMars-like conditions.

Once again, and a fortiori because of the volume of spectra, Table 5.22 summarizes these results in terms of SNR values for $\mathrm{v} 1$ and for all cases.

\begin{tabular}{|c|c|c|c|}
\hline Point (\#) & $\begin{array}{l}\text { Laser output } \\
\text { power }(\mathrm{mW})\end{array}$ & $\begin{array}{l}\text { Irradiance } \\
\left(\mathrm{kW} / \mathrm{cm}^{2}\right)\end{array}$ & $\operatorname{SNR}(\mathbf{v} 1)$ \\
\hline \multirow{12}{*}{6} & 0.32 & 0.014 & 4.3 \\
\hline & 1.14 & 0.051 & 5.8 \\
\hline & 2.84 & 0.127 & 7.8 \\
\hline & 4.55 & 0.203 & 9.4 \\
\hline & 6.42 & 0.287 & 9.7 \\
\hline & 8.12 & 0.363 & 9.0 \\
\hline & 10.1 & 0.452 & 10.4 \\
\hline & 11.8 & 0.529 & 10.6 \\
\hline & 13.5 & 0.605 & 8.3 \\
\hline & 15.4 & 0.689 & 8.8 \\
\hline & 17.1 & 0.763 & 6.6 \\
\hline & 18.8 & 0.841 & 5.4 \\
\hline \multirow{12}{*}{7} & 0.32 & 0.014 & 3.5 \\
\hline & 1.14 & 0.051 & 5.3 \\
\hline & 2.84 & 0.127 & 7.6 \\
\hline & 4.55 & 0.203 & 10.0 \\
\hline & 6.42 & 0.287 & 10.2 \\
\hline & 8.12 & 0.363 & 7.6 \\
\hline & 10.1 & 0.452 & 6.1 \\
\hline & 11.8 & 0.529 & 5.5 \\
\hline & 13.5 & 0.605 & 3.1 \\
\hline & 15.4 & 0.689 & 0.9 \\
\hline & 17.1 & 0.763 & 1.7 \\
\hline & 18.8 & 0.841 & 1.6 \\
\hline \multirow{11}{*}{8} & 0.32 & 0.014 & 5.7 \\
\hline & 1.14 & 0.051 & 10.7 \\
\hline & 2.84 & 0.127 & 16.1 \\
\hline & 4.55 & 0.203 & 19.3 \\
\hline & 6.42 & 0.287 & 18.4 \\
\hline & 8.12 & 0.363 & 14.2 \\
\hline & 10.1 & 0.452 & 13.3 \\
\hline & 11.8 & 0.529 & 4.5 \\
\hline & 13.5 & 0.605 & 2.1 \\
\hline & 15.4 & 0.689 & 0.6 \\
\hline & 17.1 & 0.763 & 0.8 \\
\hline \multirow{3}{*}{9} & 1.14 & 0.051 & 6.1 \\
\hline & 2.84 & 0.127 & 9.4 \\
\hline & 4.55 & 0.203 & 11.6 \\
\hline
\end{tabular}




\begin{tabular}{|c|c|c|c|}
\hline \multirow{4}{*}{} & 6.42 & 0.287 & 14.1 \\
\cline { 2 - 4 } & 8.12 & 0.363 & 16.2 \\
\cline { 2 - 4 } & 10.1 & 0.452 & 12.9 \\
\cline { 2 - 4 } & 11.8 & 0.529 & 9.5 \\
\cline { 2 - 4 } & 13.5 & 0.605 & 7.9 \\
\cline { 2 - 4 } & 15.4 & 0.689 & 5.9 \\
\cline { 2 - 4 } & 17.1 & 0.763 & 4.1 \\
\cline { 2 - 4 } & 18.8 & 0.841 & 1.9 \\
\hline \multirow{6}{*}{10} & 20.7 & 0.926 & 1.5 \\
\cline { 2 - 4 } & 1.14 & 1.01 & 0.3 \\
\cline { 2 - 4 } & 2.84 & 0.051 & 5.7 \\
\cline { 2 - 4 } & 4.55 & 0.127 & 6.2 \\
\cline { 2 - 4 } & 6.42 & 0.203 & 5.3 \\
\cline { 2 - 4 } & 8.12 & 0.287 & 7.6 \\
\cline { 2 - 4 } & 10.1 & 0.363 & 9.0 \\
\cline { 2 - 4 } & 11.8 & 0.452 & 8.6 \\
\cline { 2 - 4 } & 13.5 & 0.529 & 8.4 \\
\hline
\end{tabular}

Table 5.22. - SNR values for the $v 1$ peak of jarosite in all the Earth-like previous spectra with the corresponding irradiances and associated powers.

The cells marked in green correspond to maximum SNR values. Those marked in yellow correspond to a mineral that is not jarosite. It may be another mineral below the first jarosite layers or a transformation from jarosite.

There is not an obvious relation between the irradiance at which the best spectra in terms of SNR are obtained and the SNR themselves. This correlation depends on other factors that have been previously mentioned, such as the degree of crystallization of the mineral grains and their surface-to-volume ratio. Larger and better-crystallized grains provide better spectra.

\subsubsection{Hematite}

The second sample in this method of analysis is hematite, in the specified grain size distribution.

\subsection{Engineering data}

As it was done with jarosite, pressure and temperature curves, as well as flattening performance in terms of line profiles are shown in this section. 


\subsection{Pressure and Temperature curves}

As the pressure and temperature values remain stable after reaching the desired values, only the adaptation period of time is shown in Figure 5.8.

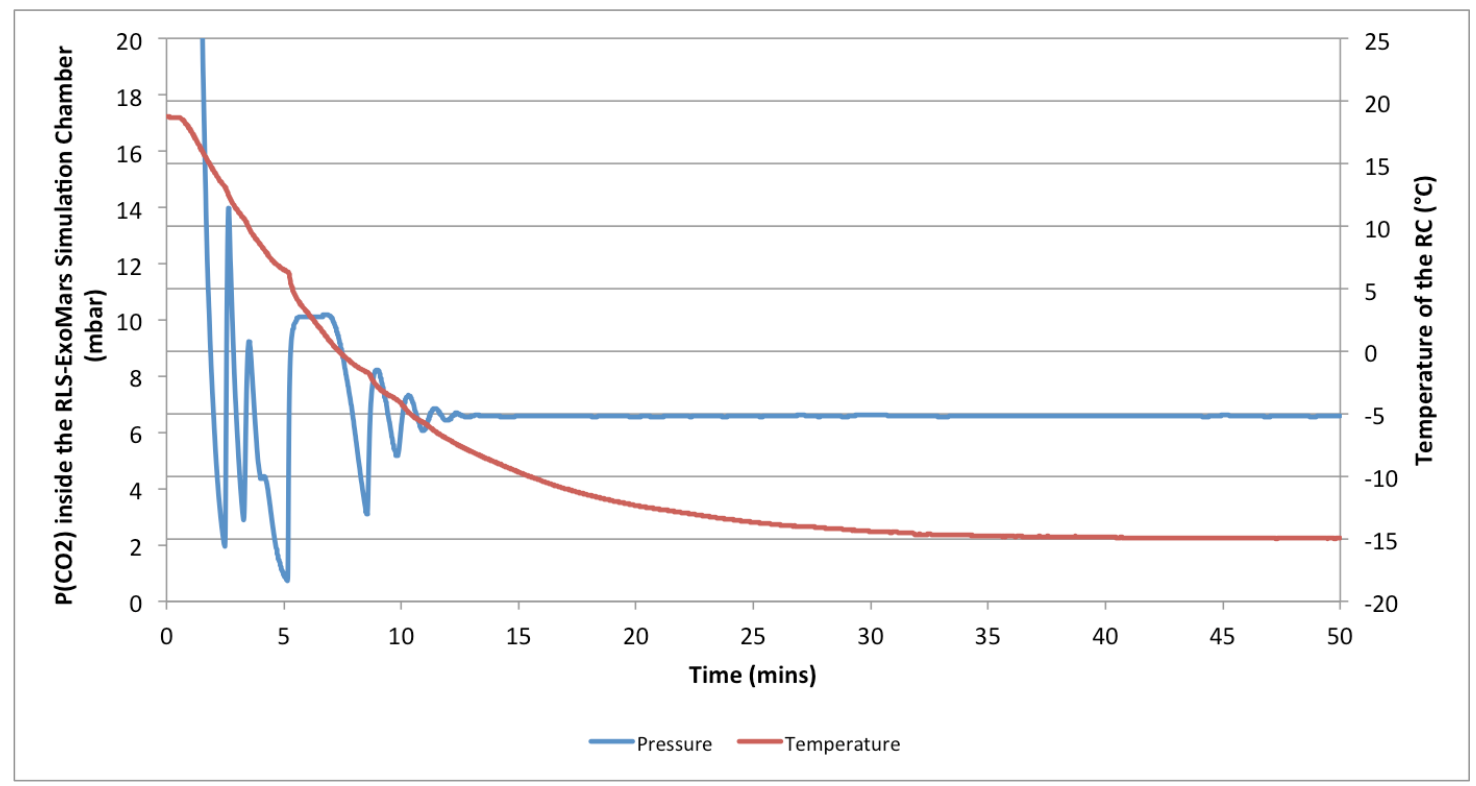

Figure 5.8. - Pressure and temperature curves for the period of achievement of the RLS-ExoMars relevant conditions. The pressure axis has been cropped to show the 'important' region.

As with the same curve for jarosite, the oscillation that the pressure curve shows is produced by the internal PID system of the pressure controller acting on the electro-actuated gas flow valve. It shows a temporary and anecdotic misbehaviour of the system, from which it recovers quickly.

Around 45 minutes from the moment the desired conditions are commanded, the system is ready to begin with the scientific operation.

\subsection{Flattening performance}

In Figure 5.9, two images from before closing and after opening the $R L S$ ExoMars Simulation Chamber with hematite on the $\mathrm{RC}$, where it is flattened under RLS-ExoMars relevant conditions. 


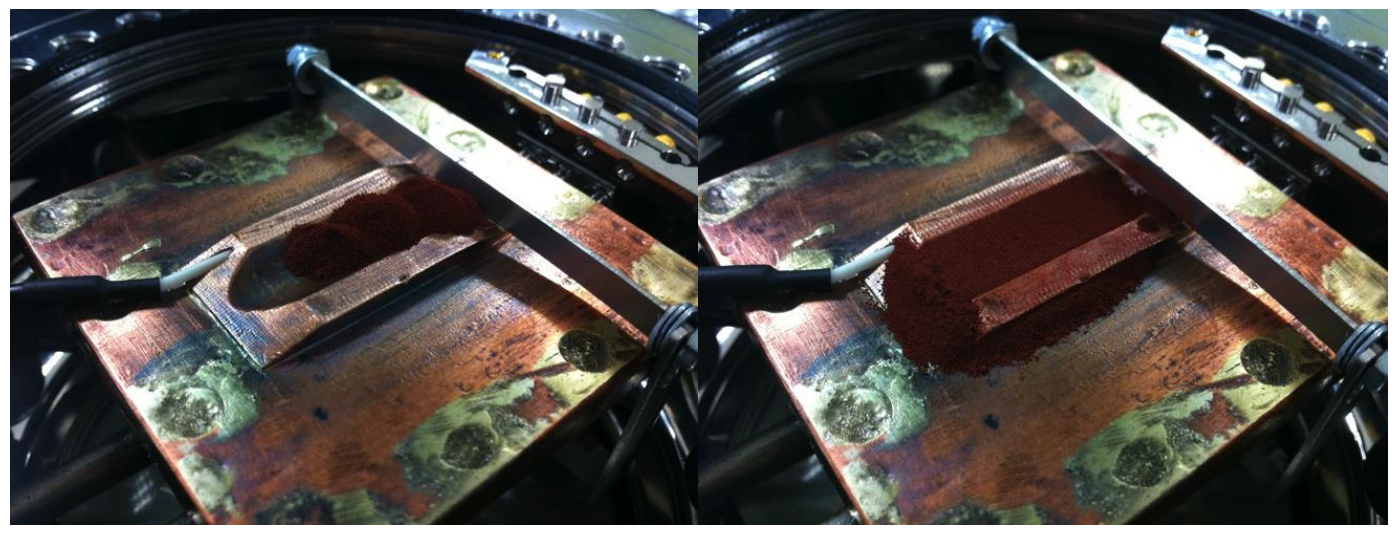

Figure 5.9. - Hematite before (left) and after (right) the flattening process inside the RLS-ExoMars Chamber.

Figure 5.10 shows the vertical profile for three central lines of the RC.

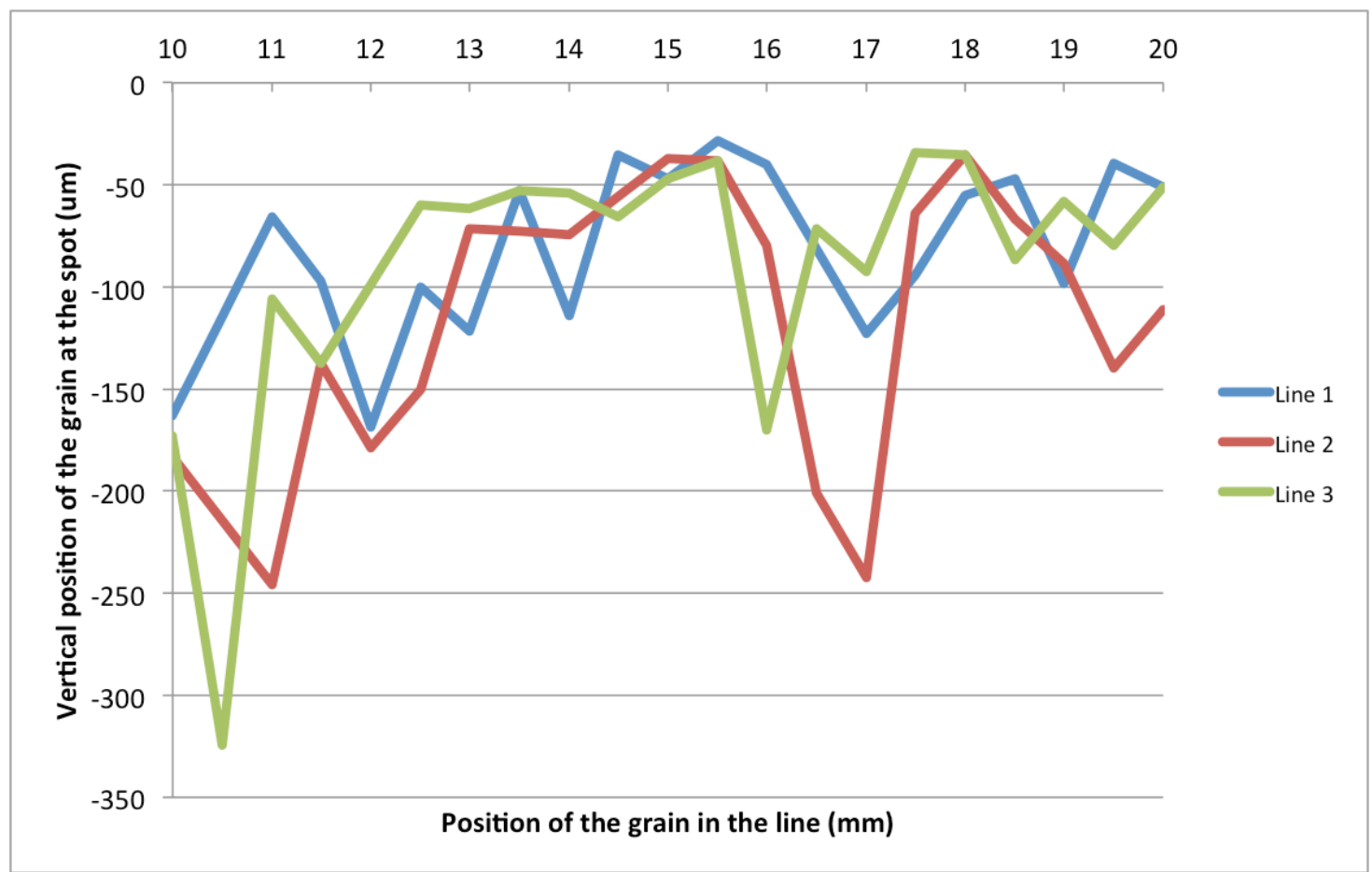

Figure 5.10. - Vertical profile of three lines in the central region of the RC.

Table 5.23 shows the statistic values for those three lines. It can be seen that all three lines comply with the requirement ${ }^{3}$. We can thus add that the flattening performance for hematite is also satisfactory. 


\begin{tabular}{|c|c|c|c|}
\cline { 2 - 4 } \multicolumn{1}{c|}{} & Line 1 & Line 2 & Line 3 \\
\hline$<100$ & $67 \%$ & $52 \%$ & $76 \%$ \\
\hline 200 & $100 \%$ & $81 \%$ & $95 \%$ \\
\hline Mean height $(\mu \mathrm{m})$ & -83 & -118 & -90 \\
\hline Desvest $(\mu \mathrm{m})$ & 41 & 69 & 67 \\
\hline
\end{tabular}

Table 5.23. - Percentage of points within $100 \mu \mathrm{m}$ and $200 \mu \mathrm{m}$ as well as mean height and standard deviation for the grains in the three profiled lines

\subsubsection{Results and Discussion}

Table 5.24 to Table 5.28 show the results for hematite analysed under RLSExoMars relevant conditions. They present the collection of spectra acquired for different irradiances, and the images taken after each spectrum, ordered in increasing irradiance. 


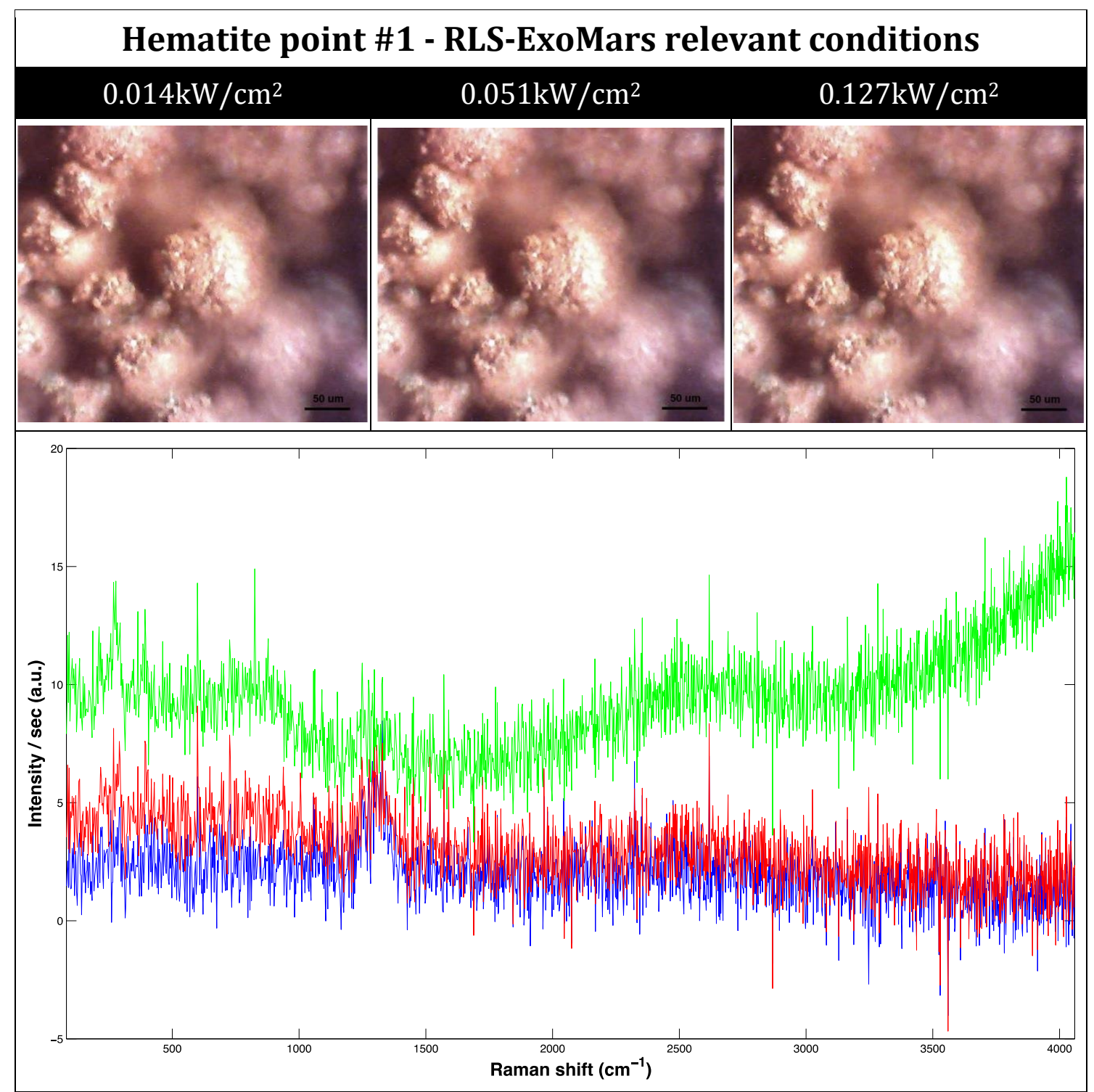

Table 5.24. - Irradiance values, images after exposure to laser and corresponding Raman spectra for the selected point \#1. Blue:0.014kW/ $\mathrm{cm}^{2}$; Red:0.051kW/ $\mathrm{cm}^{2}$; Green:0.127kW $/ \mathrm{cm}^{2}$. 


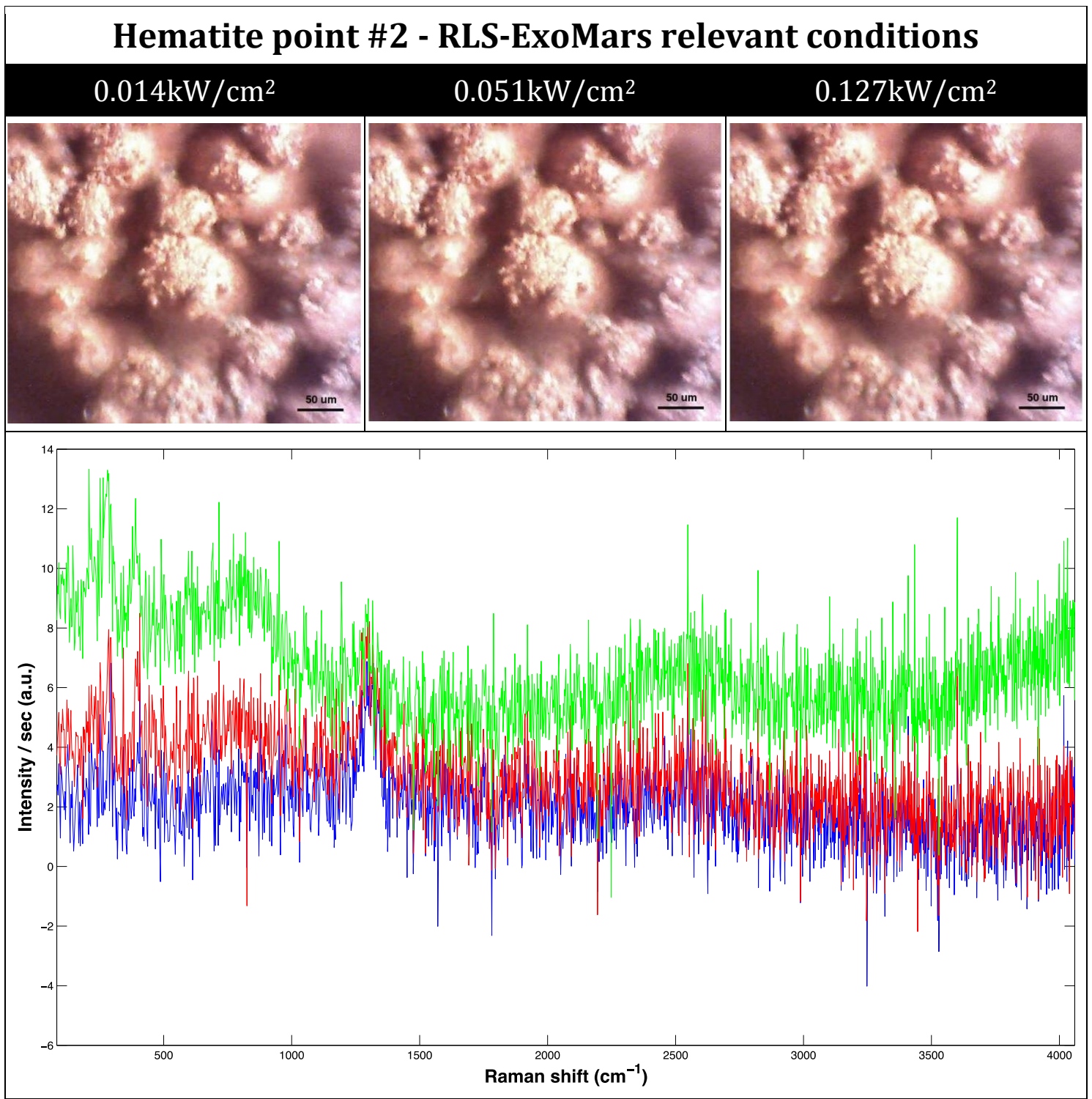

Table 5.25. - Irradiance values, images after exposure to laser and corresponding Raman spectra for the selected point \#2. Blue:0.014kW/ $\mathrm{cm}^{2}$; Red:0.051kW/ $\mathrm{cm}^{2}$; Green:0.127kW $/ \mathrm{cm}^{2}$. 


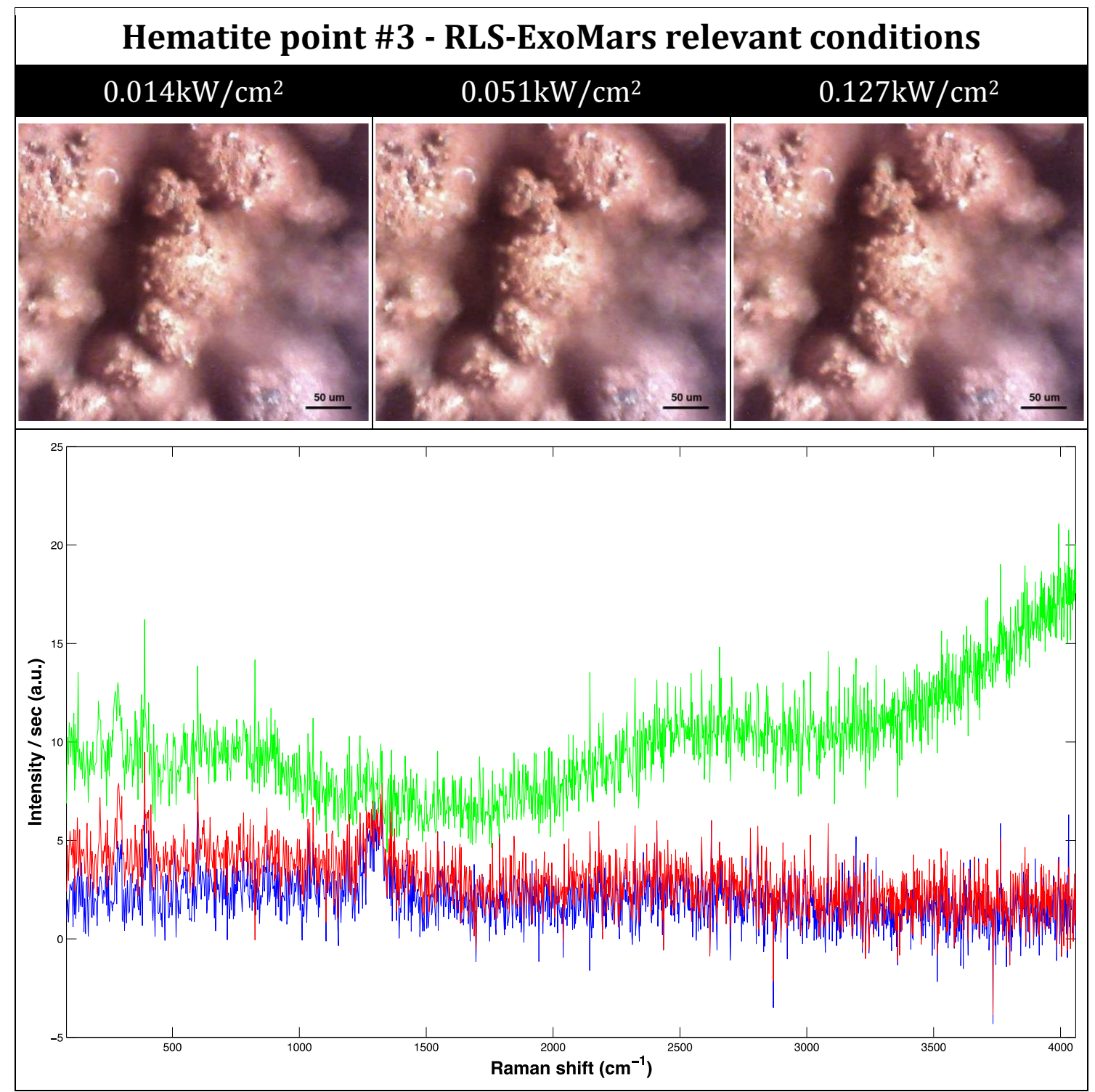

Table 5.26. - Irradiance values, images after exposure to laser and corresponding Raman spectra for the selected point \#3. Blue:0.014kW/ $\mathrm{cm}^{2}$; Red:0.051kW/ $\mathrm{cm}^{2}$; Green:0.127kW $/ \mathrm{cm}^{2}$. 


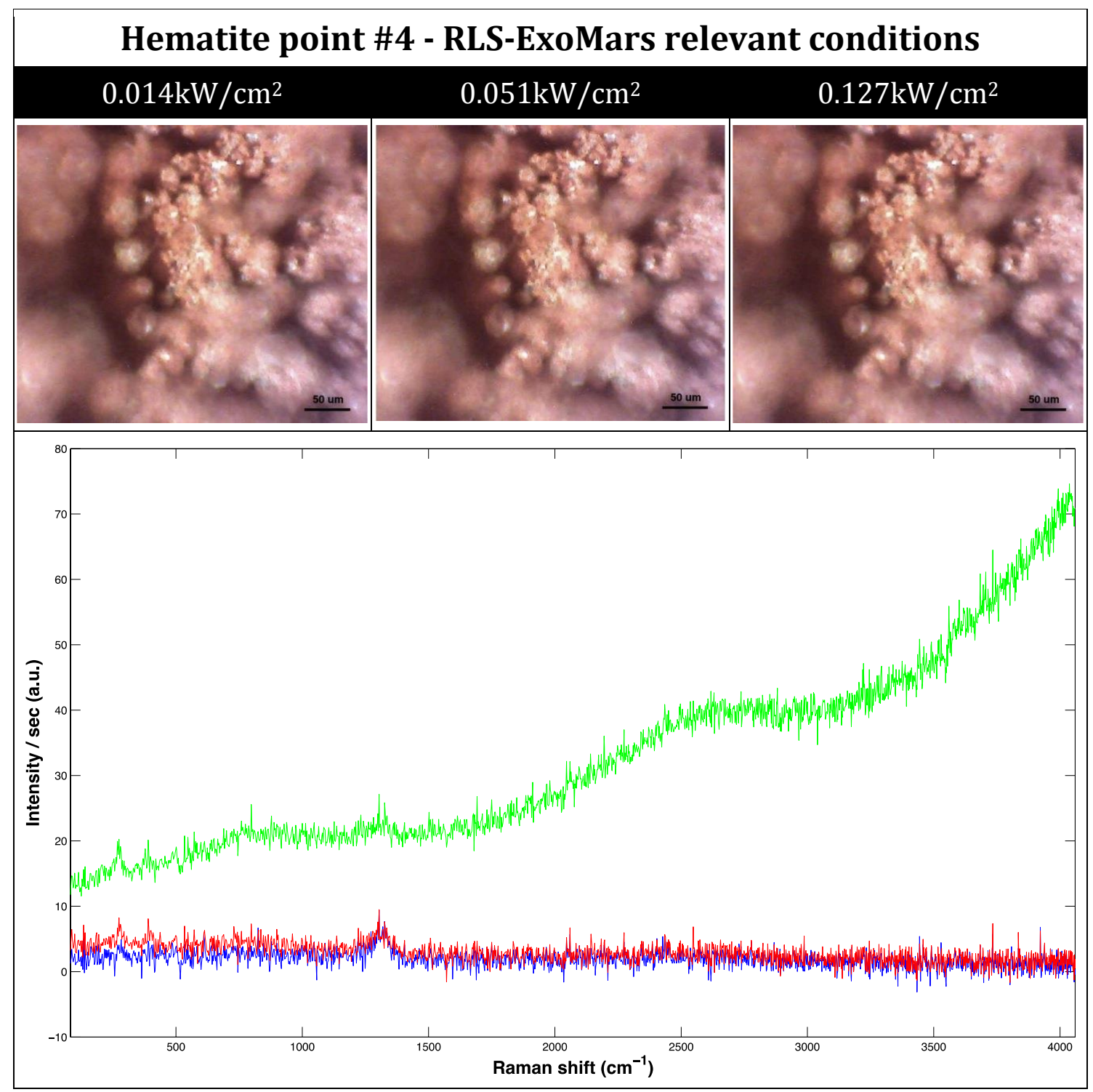

Table 5.27. - Irradiance values, images after exposure to laser and corresponding Raman spectra for the selected point \#4. Blue:0.014kW/ $\mathrm{cm}^{2}$; Red:0.051kW/cm²; Green:0.127kW $/ \mathrm{cm}^{2}$. 


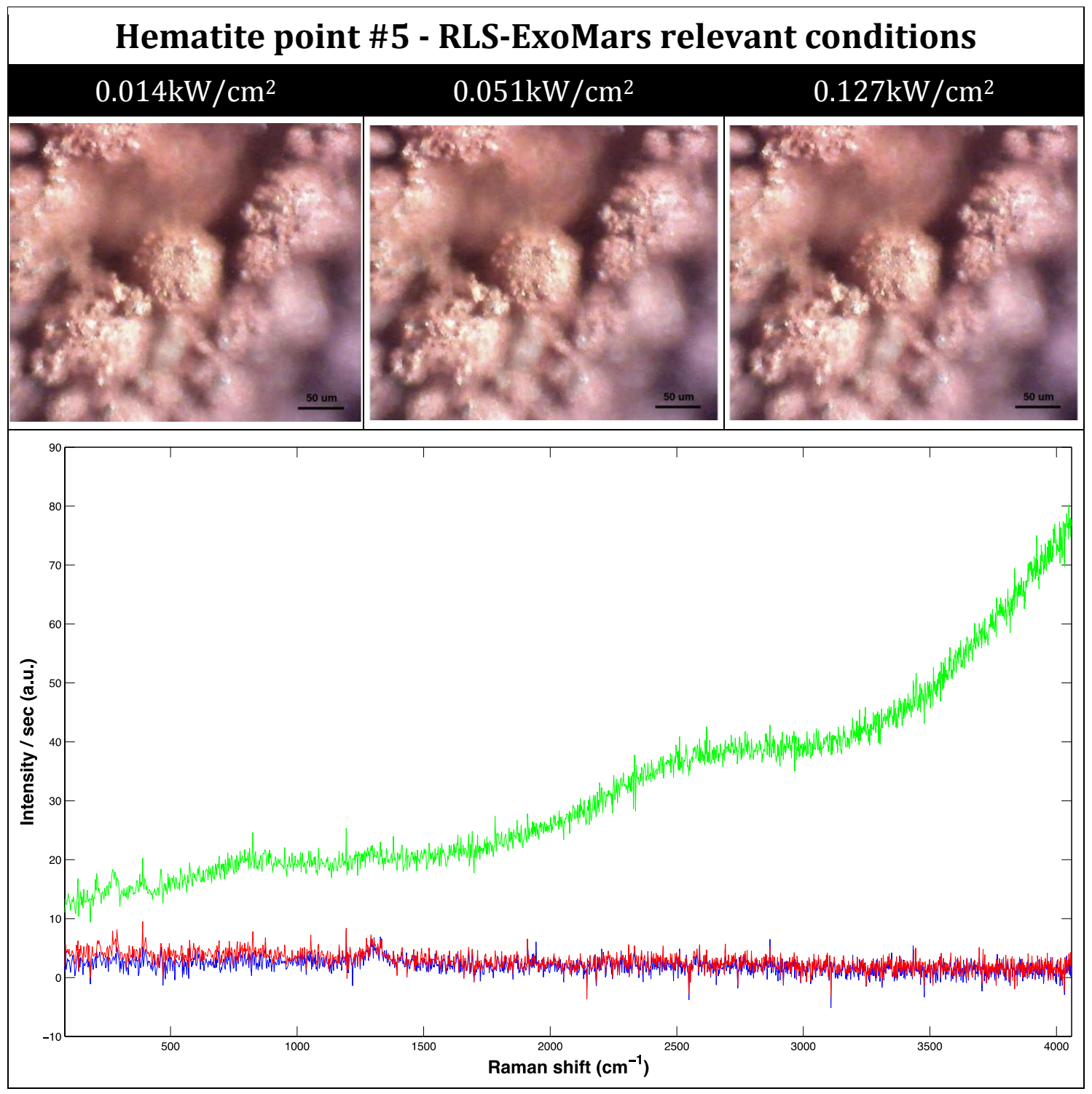

Table 5.28. - Irradiance values, images after exposure to laser and corresponding Raman spectra for the selected point \#5. Blue:0.014kW/ $\mathrm{cm}^{2}$; Red:0.051kW/ $\mathrm{cm}^{2}$; Green:0.127kW/ $\mathrm{cm}^{2}$.

Visually, the previous figures do not show any marks on the mineral grains that could be associated to thermal effects derived from the use of a laser. For images taken at higher irradiances this effect becomes a little more evident. However, due to saturation of the spectra, they are not relevant and not shown here.

Hematite is not a good Raman scatterer. In addition, under Martian pressures, the spectra are noticed to be worse compared to those obtained at room pressure. As a consequence of these facts, and combined with the use of a spectrometer with a cooled CCD to no less than $14^{\circ} \mathrm{C}$, the spectra acquired 
generally display poor quality. The following table (Table 5.29) shows the calculated SNR values for the $405 \mathrm{~cm}^{-1}$ peak of hematite.

The SNR is here calculated, as with the jarosite, as the amplitude from the baseline of the peak at $405 \mathrm{~cm}^{-1}$ divided by the noise at the baseline-subtracted peak-free $200 \mathrm{~cm}^{-1}$-wide region from 1400 to $1600 \mathrm{~cm}^{-1}$. Once again, the way this parameter is calculated here has been chosen for convenience due to the thermolability of the samples. It is intended just to compare among spectra and shall not be taken as a general method for the SNR calculation.

\begin{tabular}{|c|c|c|c|}
\hline \multirow{2}{*}{ Point $(\#)$} & \multicolumn{1}{c}{$\begin{array}{c}\text { Laser output } \\
\text { power }(\mathrm{mW})\end{array}$} & $\begin{array}{c}\text { Irradiance } \\
\left(\mathrm{kW} / \mathrm{cm}^{2}\right)\end{array}$ & SNR(405 $\mathrm{cm}^{-1}$ peak $)$ \\
\hline \multirow{3}{*}{1} & 0.32 & 0.014 & 2.45 \\
\cline { 2 - 4 } & 1.14 & 0.051 & 3.07 \\
\hline \multirow{4}{*}{2} & 2.84 & 0.127 & 4.25 \\
\hline \multirow{4}{*}{3} & 0.32 & 0.014 & 4.57 \\
\cline { 2 - 4 } & 1.14 & 0.051 & 5.16 \\
\hline \multirow{4}{*}{4} & 2.84 & 0.127 & 4.38 \\
\cline { 2 - 4 } & 0.32 & 0.014 & 5.27 \\
\hline \multirow{3}{*}{5} & 1.14 & 0.051 & 6.96 \\
\hline & 2.84 & 0.127 & 8.81 \\
\hline & 0.32 & 0.014 & 3.29 \\
\hline & 1.14 & 0.051 & 5.58 \\
\hline & 2.84 & 0.127 & 4.34 \\
\hline & 0.32 & 0.014 & 7.20 \\
\hline
\end{tabular}

Table 5.29. - SNR values, irradiances and associated powers for the $405 \mathrm{~cm}^{-1}$ peak of hematite in all the RLS-ExoMars-like previous spectra. Cells marked in green show the best SNR values.

Table 5.30 to Table 5.34 present irradiance values used to acquire spectra under Earth lab conditions, images after acquisitions, and spectra at those irradiances. 


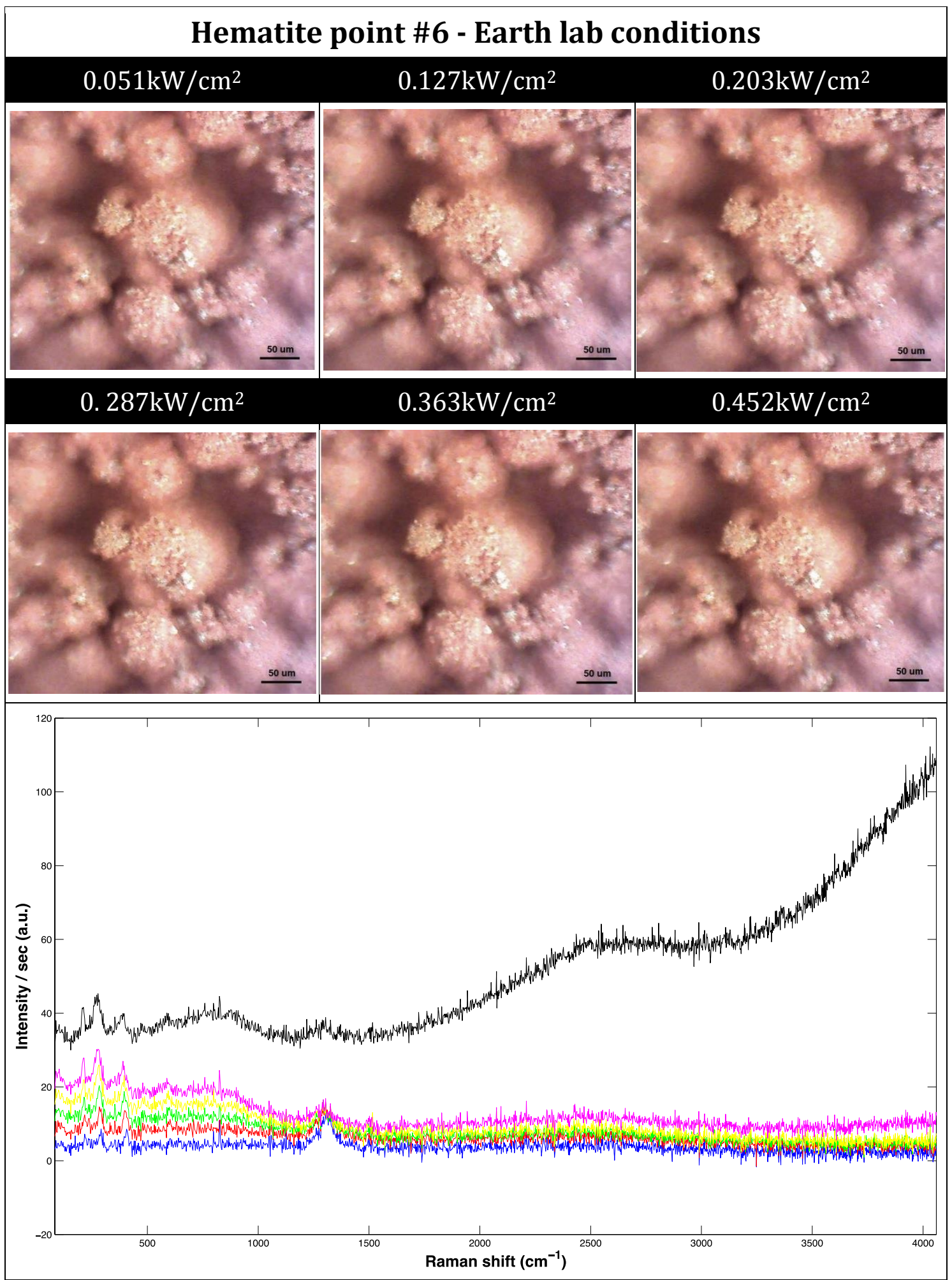

Table 5.30. - Irradiance values, images after exposure to laser and corresponding Raman spectra for the selected point \#6. Blue:0.051kW/ $/ \mathrm{cm}^{2}$; Red:0.127kW/ $\mathrm{cm}^{2}$; Green:0.203kW $/ \mathrm{cm}^{2}$; Yellow:0.287kW/ $\mathrm{cm}^{2}$; Magenta:0.363kW/ $\mathrm{cm}^{2}$; Black:0.452kW $/ \mathrm{cm}^{2}$. 


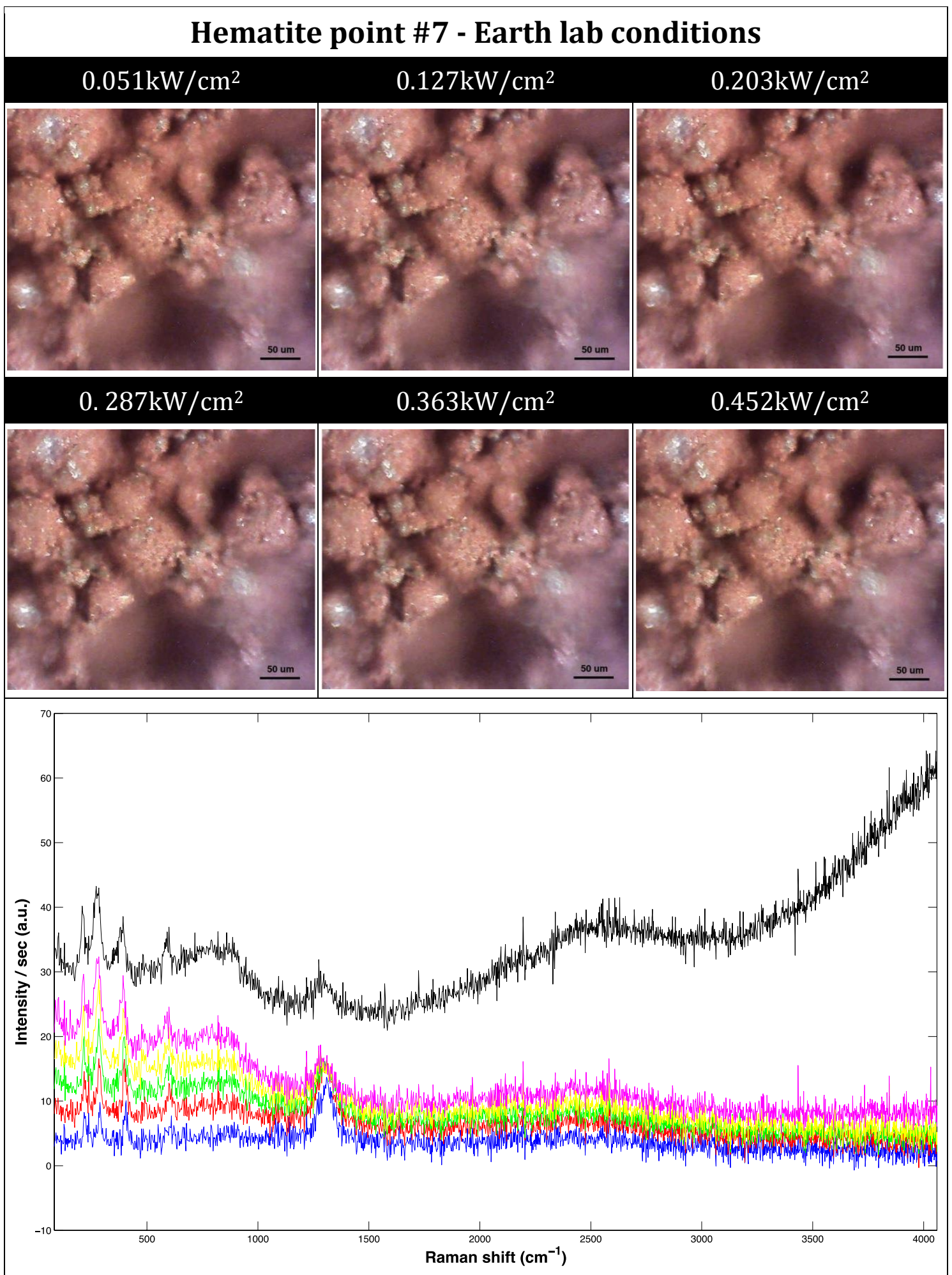

Table 5.31. - Irradiance values, images after exposure to laser and corresponding Raman spectra for the selected point \#7. Blue:0.051kW/ $\mathrm{cm}^{2}$; Red:0.127kW/ $\mathrm{cm}^{2}$; Green:0.203kW $/ \mathrm{cm}^{2}$; Yellow:0.287kW $/ \mathrm{cm}^{2}$; Magenta:0.363kW/ $/ \mathrm{cm}^{2}$; Black:0.452kW $/ \mathrm{cm}^{2}$. 


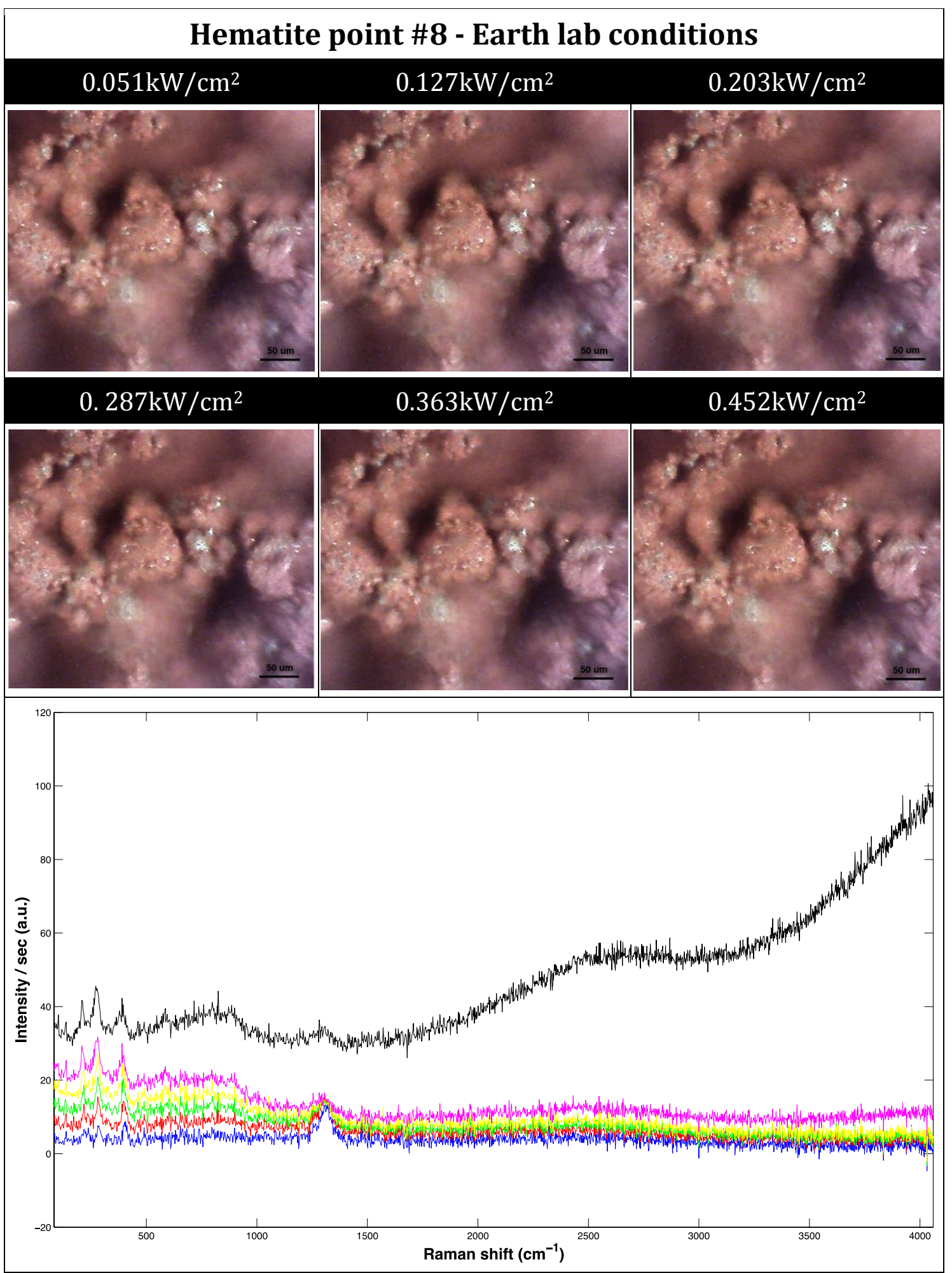

Table 5.32. - Irradiance values, images after exposure to laser and corresponding Raman spectra for the selected point \#8. Blue:0.051kW/ $\mathrm{cm}^{2}$; Red:0.127kW/ $\mathrm{cm}^{2}$; Green:0.203kW $/ \mathrm{cm}^{2}$; Yellow:0.287kW $/ \mathrm{cm}^{2}$; Magenta:0.363kW/ $\mathrm{cm}^{2}$; Black:0.452kW/ $\mathrm{cm}^{2}$. 


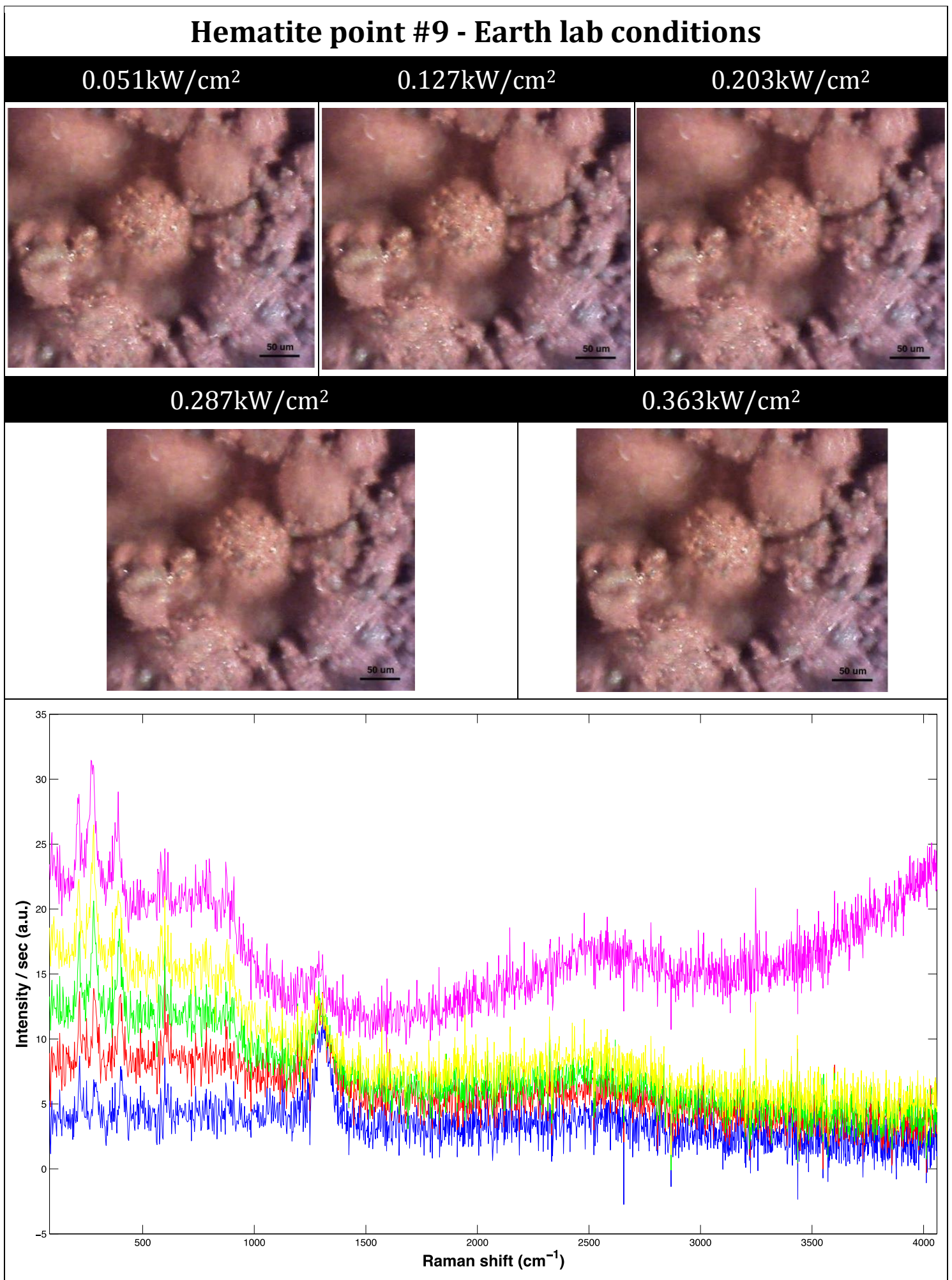

Table 5.33. - Irradiance values, images after exposure to laser and corresponding Raman spectra for the selected point \#9. Blue:0.051kW/ $\mathrm{cm}^{2}$; Red:0.127kW/ $\mathrm{cm}^{2}$; Green:0.203kW $/ \mathrm{cm}^{2}$; Yellow:0.287kW $/ \mathrm{cm}^{2}$; Magenta:0.363kW/ $\mathrm{cm}^{2}$. 


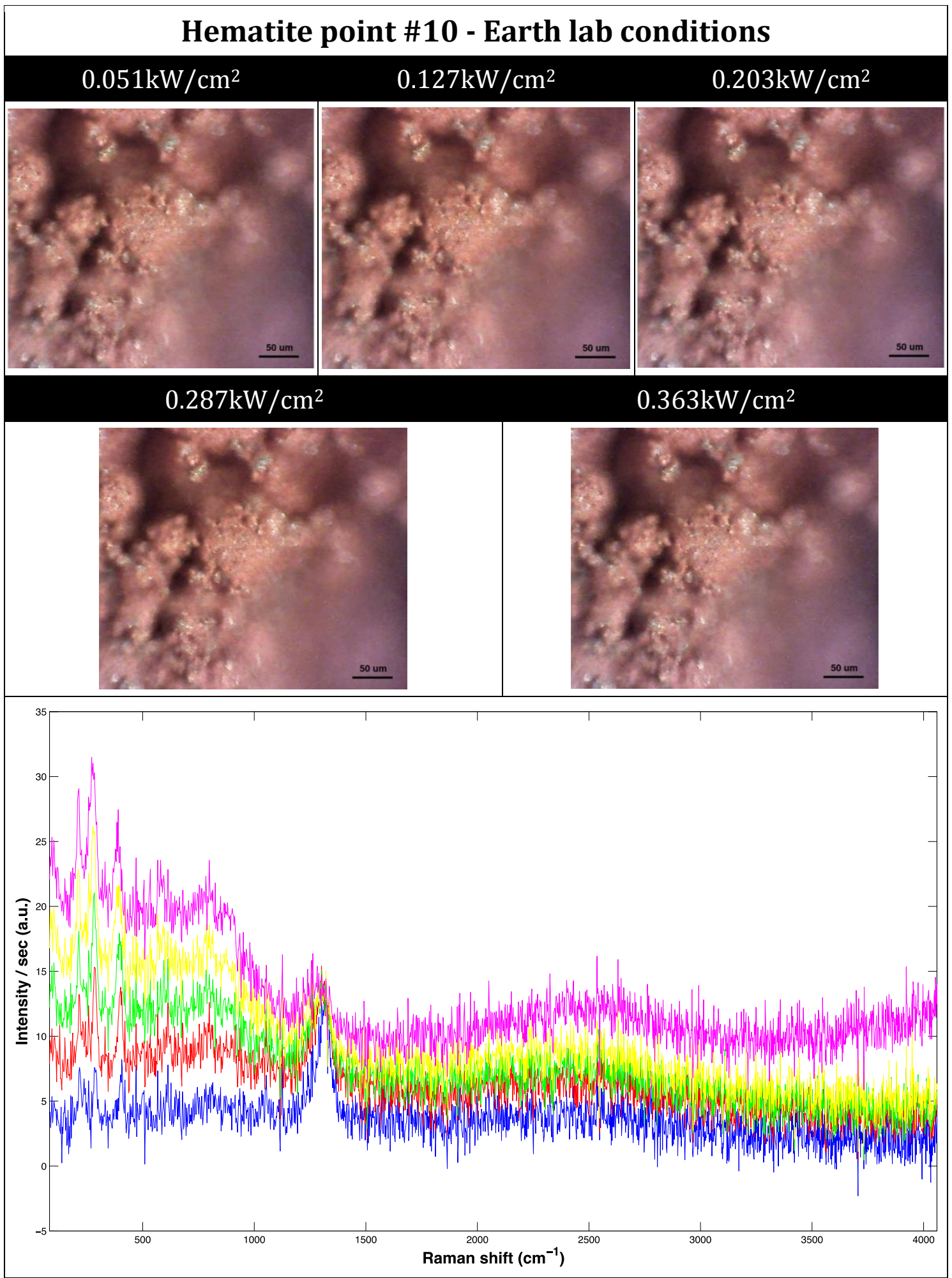

Table 5.34. - Irradiance values, images after exposure to laser and corresponding Raman spectra for the selected point \#10. Blue:0.051kW/ $\mathrm{cm}^{2}$; Red:0.127kW/ $\mathrm{cm}^{2}$; Green:0.203kW $/ \mathrm{cm}^{2}$; Yellow:0.287kW $/ \mathrm{cm}^{2}$; Magenta:0.363kW/ $\mathrm{cm}^{2}$. 
As noticed for hematite under RLS-ExoMars relevant conditions, the mineral grains lack of a visual signature of damage at these irradiances, where the tail has not yet raised or begins to.

Spectroscopically speaking, the hematite spectra under Earth lab conditions get better in terms of SNR as the following Table 5.35 shows. The evolution of the $405 \mathrm{~cm}^{-1}$ Raman peak of hematite for Earth lab conditions is here presented.

\begin{tabular}{|c|c|c|c|}
\hline Point (\#) & $\begin{array}{l}\text { Laser output } \\
\text { power }(\mathrm{mW})\end{array}$ & $\begin{array}{l}\text { Irradiance } \\
\left(\mathrm{kW} / \mathrm{cm}^{2}\right)\end{array}$ & $\operatorname{SNR}\left(405 \mathrm{~cm}^{-1}\right.$ peak $)$ \\
\hline \multirow{6}{*}{6} & 1.14 & 0.051 & 5.63 \\
\hline & 2.84 & 0.127 & 6.45 \\
\hline & 4.55 & 0.203 & 7.41 \\
\hline & 6.42 & 0.287 & 6.73 \\
\hline & 8.12 & 0.363 & 7.92 \\
\hline & 10.1 & 0.452 & 5.07 \\
\hline \multirow{6}{*}{7} & 1.14 & 0.051 & 6.87 \\
\hline & 2.84 & 0.127 & 8.92 \\
\hline & 4.55 & 0.203 & 8.43 \\
\hline & 6.42 & 0.287 & 9.19 \\
\hline & 8.12 & 0.363 & 9.71 \\
\hline & 10.1 & 0.452 & 7.31 \\
\hline \multirow{6}{*}{8} & 1.14 & 0.051 & 5.39 \\
\hline & 2.84 & 0.127 & 7.59 \\
\hline & 4.55 & 0.203 & 10.39 \\
\hline & 6.42 & 0.287 & 8.57 \\
\hline & 8.12 & 0.363 & 10.40 \\
\hline & 10.1 & 0.452 & 7.98 \\
\hline \multirow{5}{*}{9} & 1.14 & 0.051 & 4.71 \\
\hline & 2.84 & 0.127 & 5.86 \\
\hline & 4.55 & 0.203 & 7.97 \\
\hline & 6.42 & 0.287 & 5.82 \\
\hline & 8.12 & 0.363 & 9.34 \\
\hline \multirow{5}{*}{10} & 1.14 & 0.051 & 4.92 \\
\hline & 2.84 & 0.127 & 5.84 \\
\hline & 4.55 & 0.203 & 7.75 \\
\hline & 6.42 & 0.287 & 6.69 \\
\hline & 8.12 & 0.363 & 7.88 \\
\hline
\end{tabular}

Table 5.35. - SNR values for the $405 \mathrm{~cm}^{-1}$ peak of hematite in all the Earth-like previous spectra.

Comparing the thermolability in this case with the previous one, under RLSExoMars conditions, the mineral grains bear more irradiance and, at the optimum, the SNR doubles in value. 


\subsection{Experiment 3: Sweep in spot size}

This second science test seeks to find the minimum spot size at which, maintaining a constant laser power (the one that gives in focus the minimum irradiance for RLS-ExoMars: $0.6 \mathrm{~kW} / \mathrm{cm}^{2}$ ), the mineral grains of our selected samples get affected by thermal effects derived from exposure to laser light.

Defocusing both the excitation and collection by moving the optical head upwards from the focusing point on each grain produces a bigger spot size and so a lower irradiance with which to excite the sample. As it is a confocal optical system, defocusing the excitation path to lower the irradiance on the sample means defocusing the collection path getting weaker Raman signals.

Moreover, the NA of our RLS-ExoMars Science Simulator is 0.40, larger than the 0.22 of the real instrument. This characteristic in the real instrument should only affect in the amount of collected light, the DOF and the distance it has to move upwards in case it is needed to reduce irradiance, what is being tested here. In the case of our optical system, this distance should be smaller.

As for the previous experiment, this one was also performed in both RLSExoMars relevant conditions $\left(\mathrm{T}=-15^{\circ} \mathrm{C}\right.$ and $\left.\mathrm{p}_{\mathrm{CO} 2} \approx 7.5 \mathrm{mbar}\right)$ and Earth lab conditions $\left(\mathrm{T}=20^{\circ} \mathrm{C}\right.$ and atmospheric pressure in the lab).

\subsubsection{Methodology}

This test was performed in series with the sweep in power test, taking advantage of the previously deposited and flattened sample. The next pseudocode depicts this procedure:

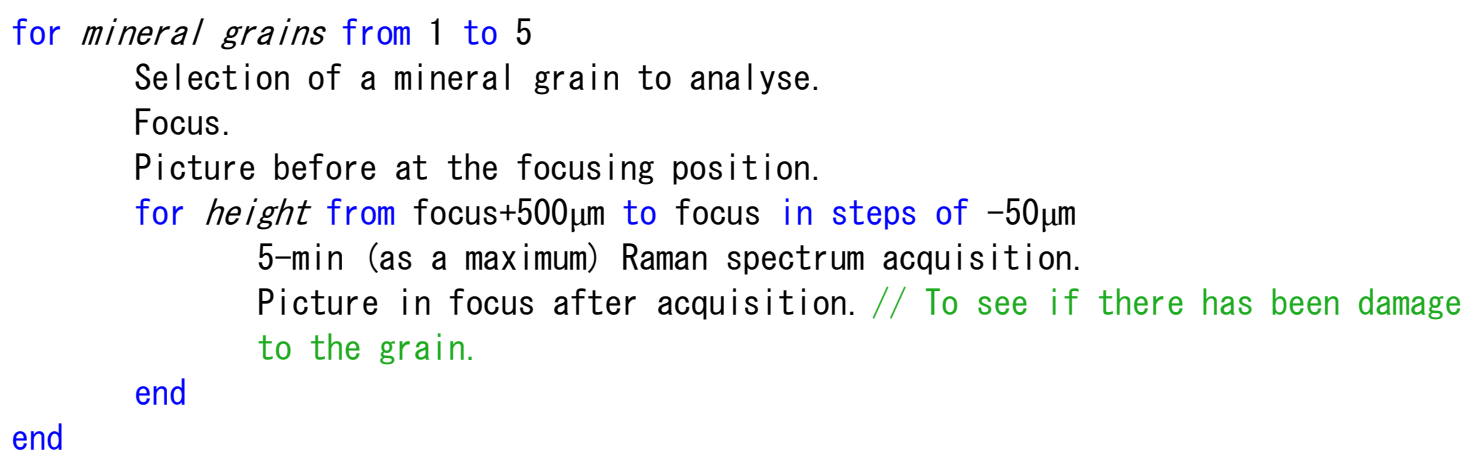


Go to Earth lab conditions. // These conditions are met for the power sweep test and also used for this one. Once again, to compare with RLS-Exollars conditions.

for mineral grains from 1 to 5

Selection of a mineral grain to analyse.

Focus.

Picture before at the focus position.

for height from focus $+500 \mu \mathrm{m}$ to focus in steps of $-50 \mu \mathrm{m}$ 5 -min (as a maximum) Raman spectrum acquisition.

Picture in focus after acquisition. // To see if there has been damage

to the grain. The scale shown in the image is $50 \mu \mathrm{m}$

end

end

\subsubsection{Samples}

\subsubsection{Jarosite}

The first sample to be analysed was jarosite in the previously specified grain size distribution.

\subsection{Engineering data}

\subsection{Pressure and Temperature curves}

In this case, there are no pressure and temperature curves as the environmental conditions are maintained after the performance of the power sweep experiment. So, for the whole development of this analysis, the pressure is kept at approximately $7.5 \mathrm{mbar}$ of $\mathrm{CO}_{2}$ partial pressure and the temperature at $15^{\circ} \mathrm{C}$.

\subsection{Flattening performance}

Neither the RC nor the sample were moved after the power sweep experiment executed before this one. So, the line profiles obtained before are still valid.

\subsection{Results and Discussion}

Table 5.36 to Table 5.40 show the visual and spectroscopic results from the analysis of jarosite at different heights from focus. In some cases it can be noticed that the grain suddenly disappears. This may be related with the expansion of gasses as the grain gets damaged or to the reduction of volume as chemical reactions are produced. 


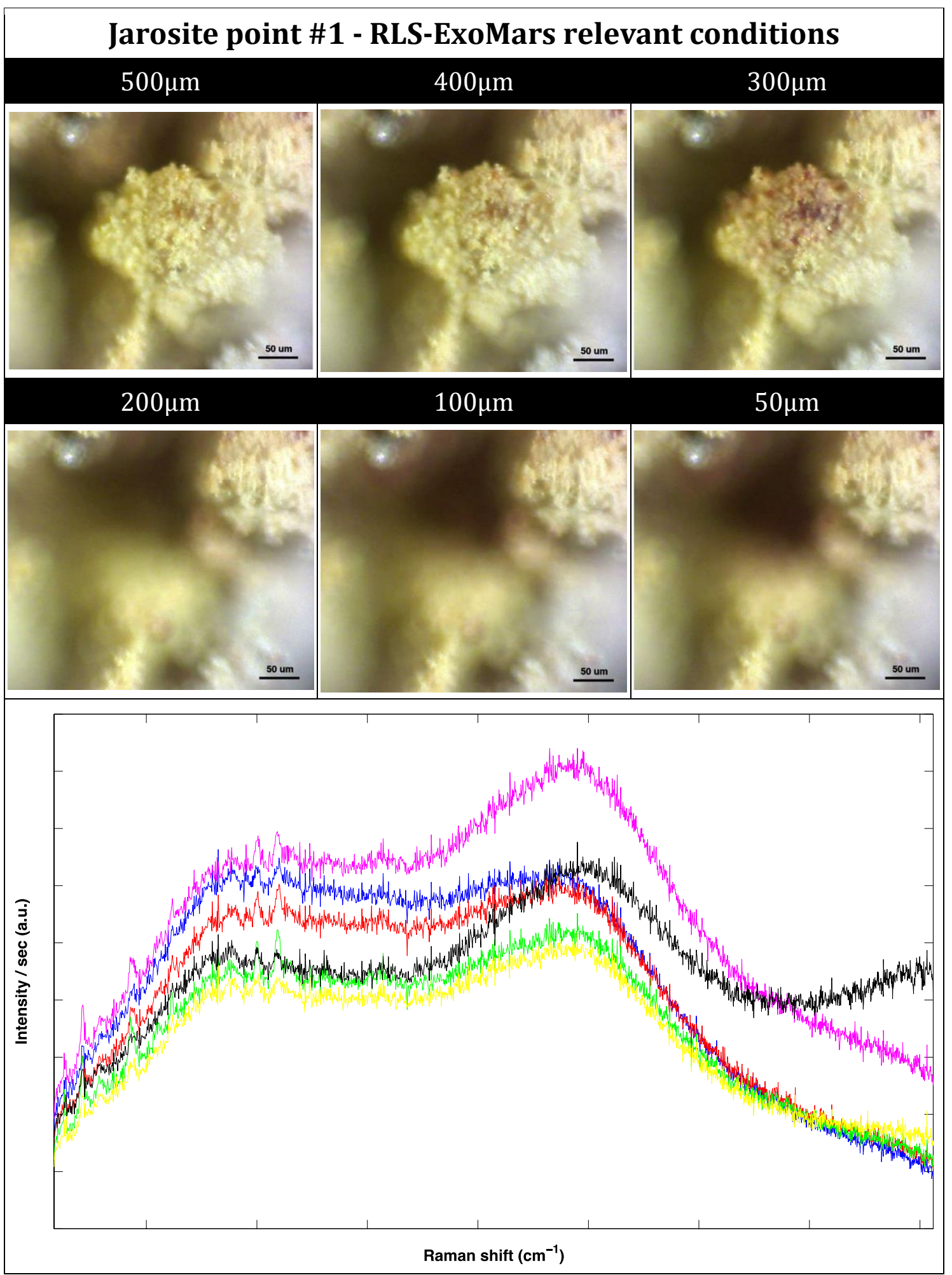

Table 5.36. - Height values from focus, images after exposure to laser and corresponding Raman spectra for the selected point \#1. Blue:500 $\mu \mathrm{m}$;

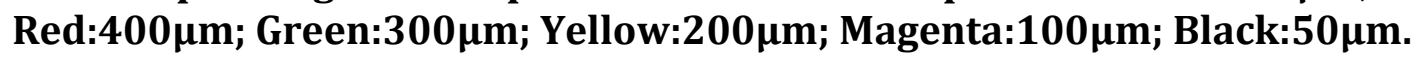




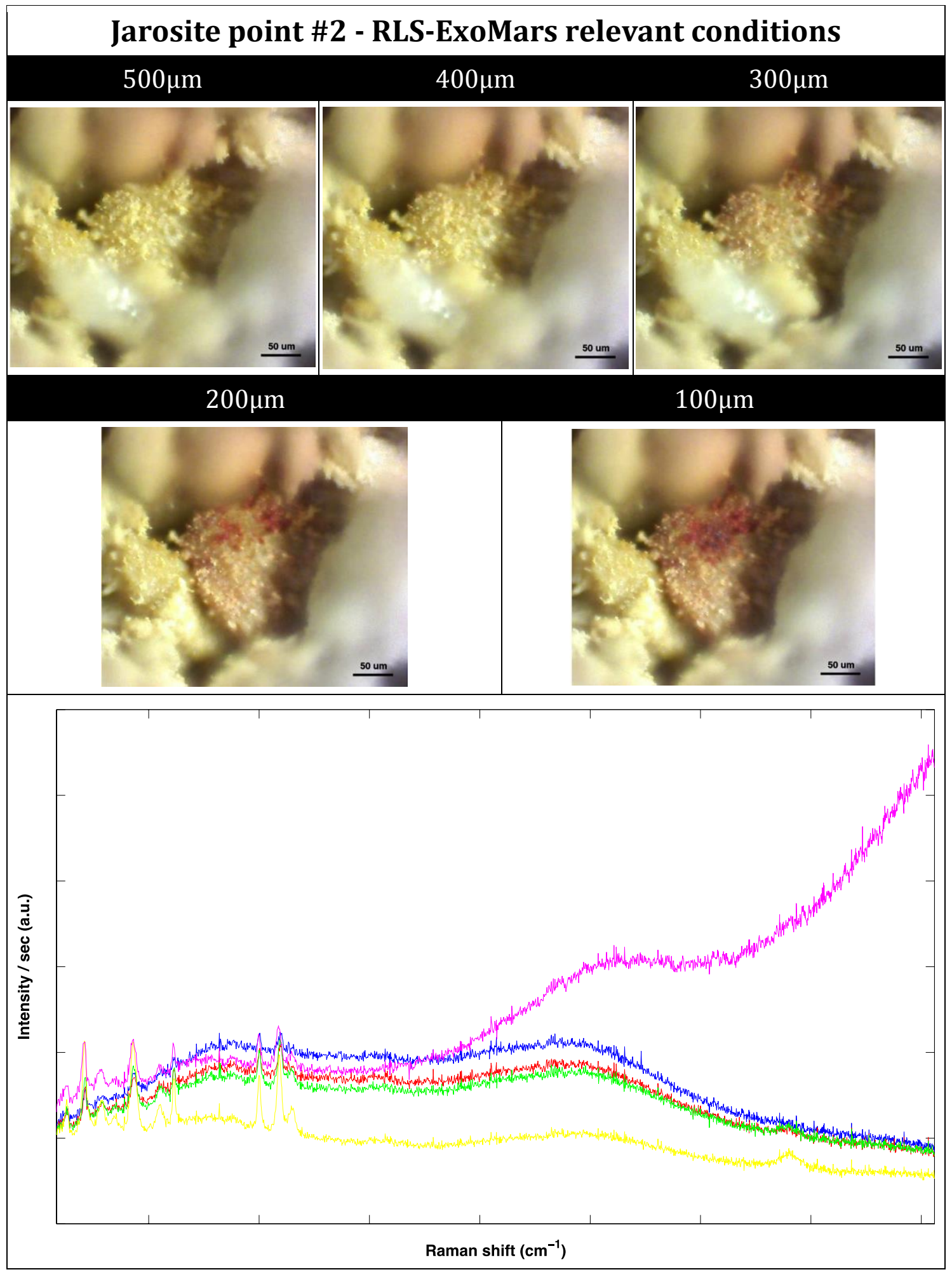

Table 5.37. - Height values from focus, images after exposure to laser and corresponding Raman spectra for the selected point \#2. Blue:500 $\mu \mathrm{m}$;

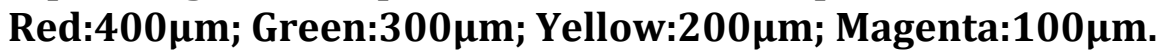




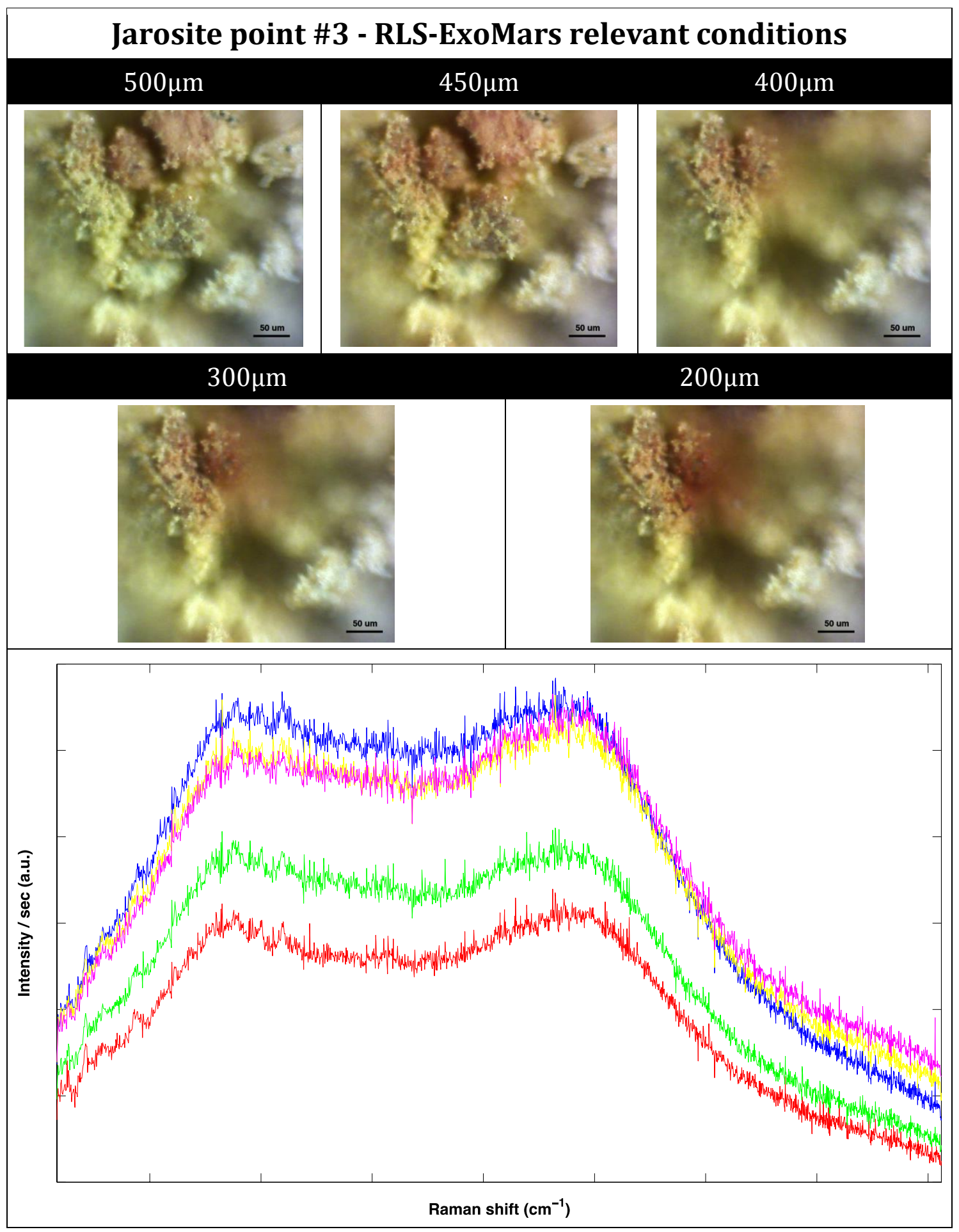

Table 5.38. - Height values from focus, images after exposure to laser and corresponding Raman spectra for the selected point \#3. Blue:500 $\mathrm{\mu m}$;

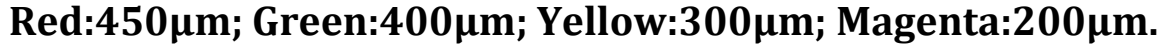




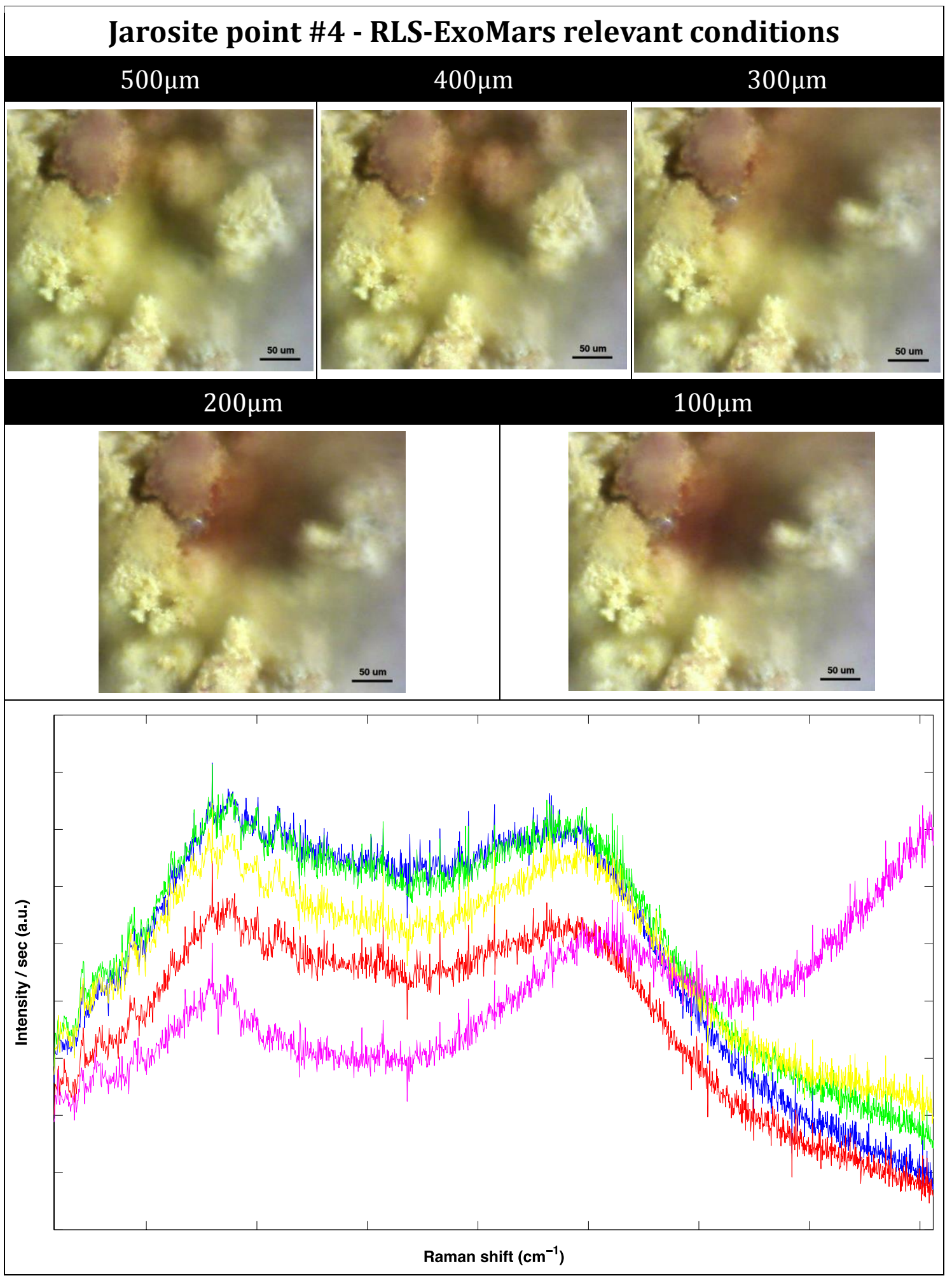

Table 5.39. - Height values from focus, images after exposure to laser and corresponding Raman spectra for the selected point \#4. Blue:500 $\mu \mathrm{m}$;

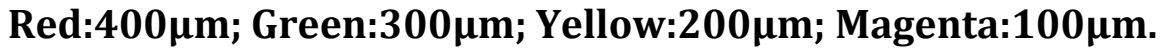




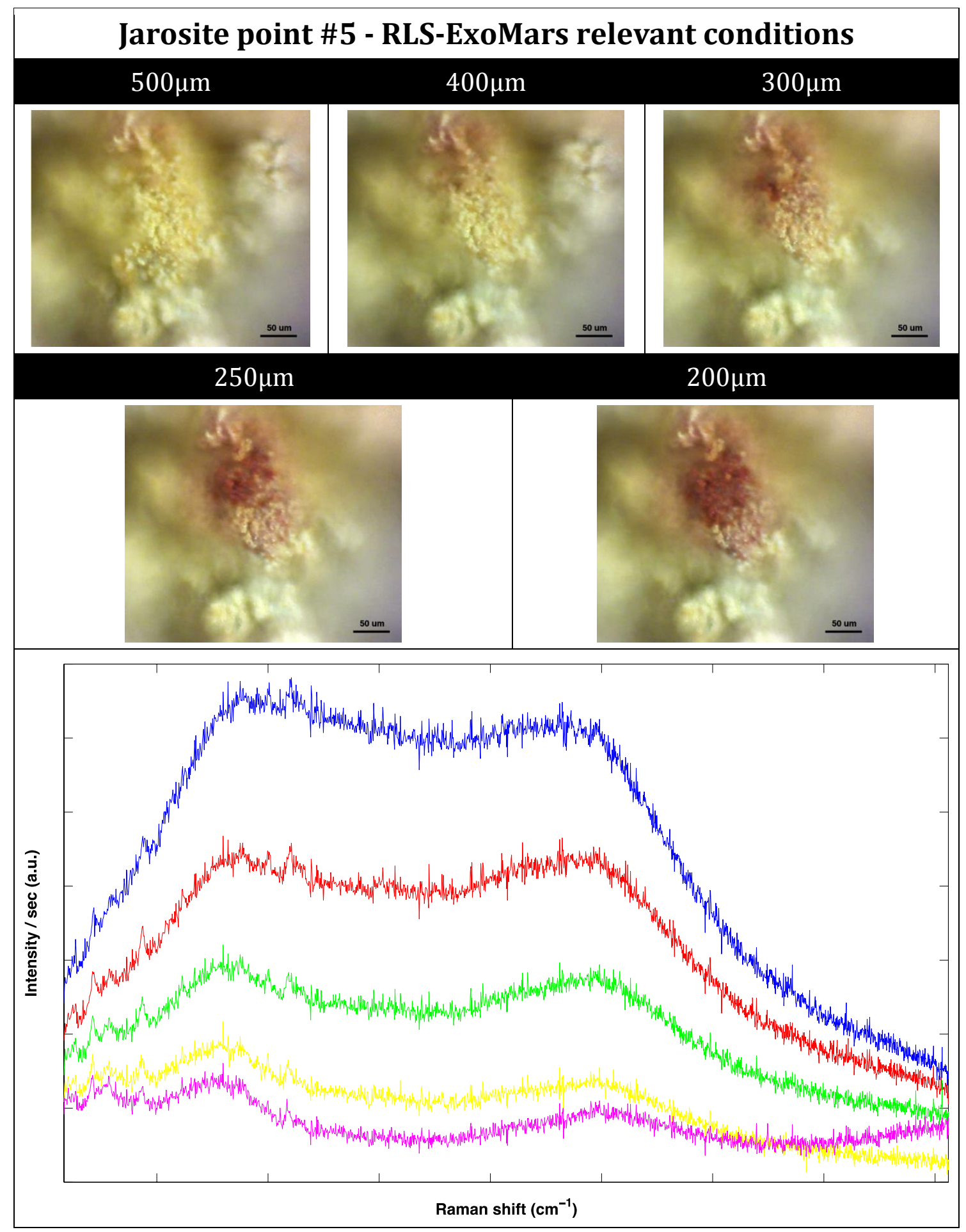

Table 5.40. - Height values from focus, images after exposure to laser and corresponding Raman spectra for the selected point \#5. Blue:500 $\mu \mathrm{m}$;

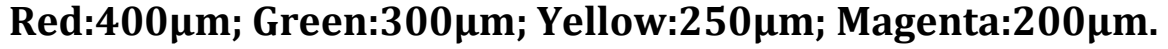


Visually, all the analysed grains of jarosite are marked with the laser at $300 \mu \mathrm{m}$ from the focus or higher. This corresponds to spot sizes over $150 \mu \mathrm{m}$. For some grains, for example the one at point \#4, the grain already disappears after exposure from a height of $+500 \mu \mathrm{m}$ from the focus.

Table 5.41 summarizes the results that previous tables present. In it, SNR values for the $v 1$ mode of jarosite (at $1000 \mathrm{~cm}^{-1}$ ) for the different acquired spectra are listed.

\begin{tabular}{|c|c|c|c|c|}
\hline Point (\#) & $\begin{array}{l}\text { Height from } \\
\text { focus }(\mu \mathrm{m})\end{array}$ & $\begin{array}{c}\text { Spot size } \\
@ 13.5 \% \\
(\mu \mathrm{m})\end{array}$ & $\begin{array}{l}\text { Irradiance } \\
\left(\mathrm{kW} / \mathrm{cm}^{2}\right)\end{array}$ & SNR(v1) \\
\hline \multirow{6}{*}{1} & 500 & 251 & 0.028 & 2.6 \\
\hline & 400 & 200 & 0.043 & 5.4 \\
\hline & 300 & 155 & 0.071 & 7.5 \\
\hline & 200 & 111 & 0.140 & 4.0 \\
\hline & 100 & 70 & 0.357 & 5.1 \\
\hline & 50 & 57 & 0.522 & 2.9 \\
\hline \multirow{5}{*}{2} & 500 & 251 & 0.028 & 7.9 \\
\hline & 400 & 200 & 0.043 & 11.6 \\
\hline & 300 & 155 & 0.071 & 16.7 \\
\hline & 200 & 111 & 0.140 & 33.2 \\
\hline & 100 & 70 & 0.357 & 13.8 \\
\hline \multirow{5}{*}{3} & 500 & 251 & 0.028 & 2.7 \\
\hline & 450 & 227 & 0.033 & 4.1 \\
\hline & 400 & 200 & 0.043 & 2.9 \\
\hline & 300 & 155 & 0.071 & 2.4 \\
\hline & 200 & 111 & 0.140 & 2.3 \\
\hline \multirow{5}{*}{4} & 500 & 251 & 0.028 & 3.9 \\
\hline & 400 & 200 & 0.043 & 3.4 \\
\hline & 300 & 155 & 0.071 & 3.1 \\
\hline & 200 & 111 & 0.140 & 2.8 \\
\hline & 100 & 70 & 0.357 & 2.2 \\
\hline \multirow{5}{*}{5} & 500 & 251 & 0.028 & 4.2 \\
\hline & 400 & 200 & 0.043 & 3.6 \\
\hline & 300 & 155 & 0.071 & 4.0 \\
\hline & 250 & 132 & 0.099 & 6.2 \\
\hline & 200 & 111 & 0.140 & 3.0 \\
\hline
\end{tabular}

Table 5.41. - SNR values for the v1 peak of jarosite in all the RLS-ExoMarslike previous spectra. Height from focus, spot size at that height and irradiance is also presented in it. Green cells mark the best SNR values for each point.

From this table it can be observed that the best spectra in terms of SNR are usually obtained coinciding with the visual boundary between a clean and a 
marked mineral grain. Mainly because many of them get displaced and out of focus after that. For grains with good thermal dissipation the spot size can be even narrower (higher irradiance) and thus the SNR can increase before losing the grain. This happened also in the "sweep in power" experiment where wellcrystallized grains endured after illuminating them with high values of irradiance.

Table 5.42 to Table 5.46 show the results for the same analyses done to jarosite mineral grains this time under Earth lab conditions $\left(\mathrm{T}=20^{\circ} \mathrm{C}\right.$ and Earth atmospheric pressure). 


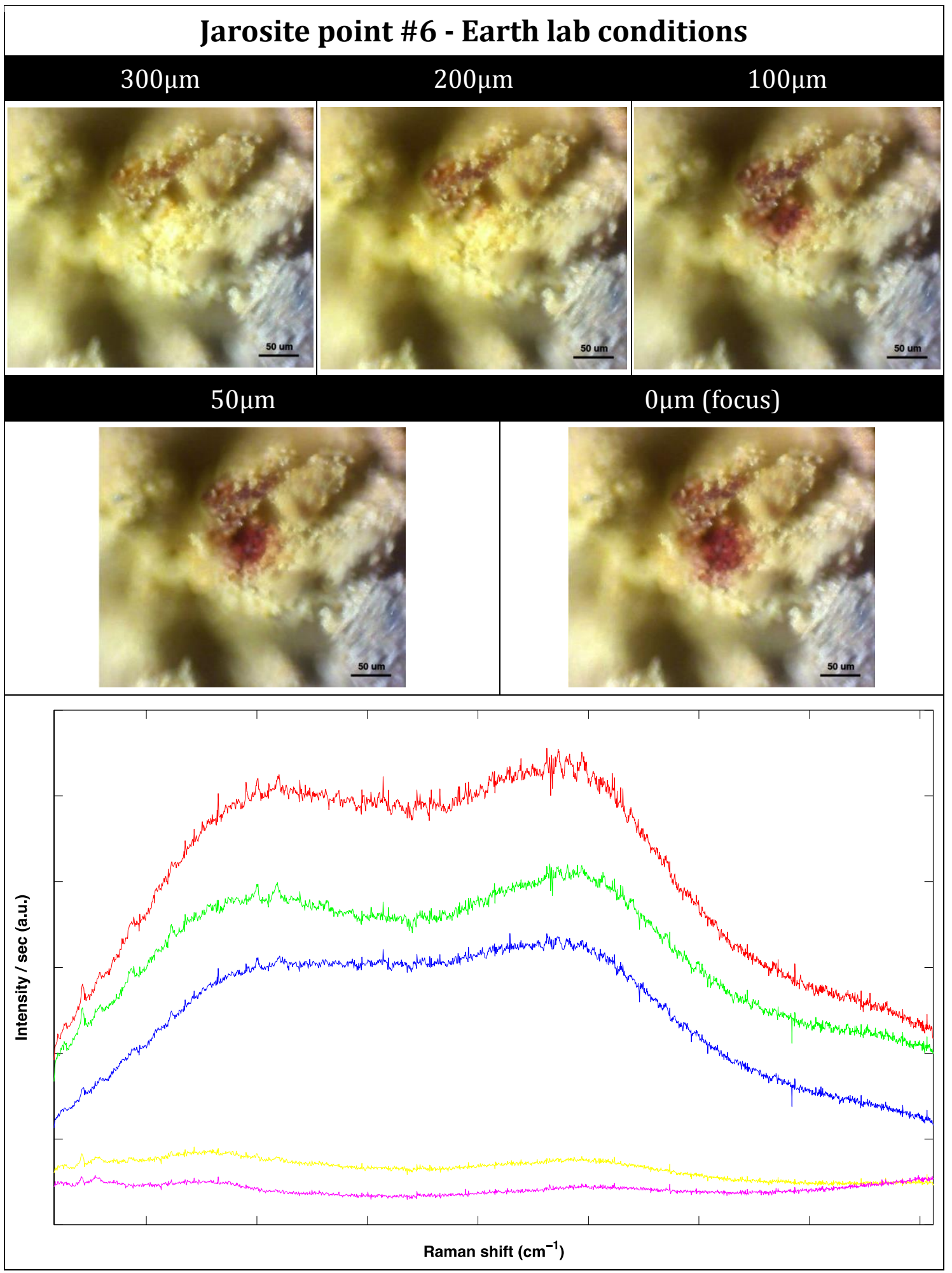

Table 5.42. - Height values from focus, images after exposure to laser and corresponding Raman spectra for the selected point \#6. Blue:300 $\mathrm{mm}$;

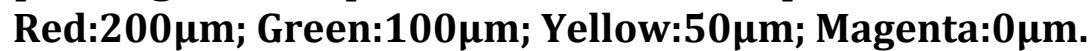




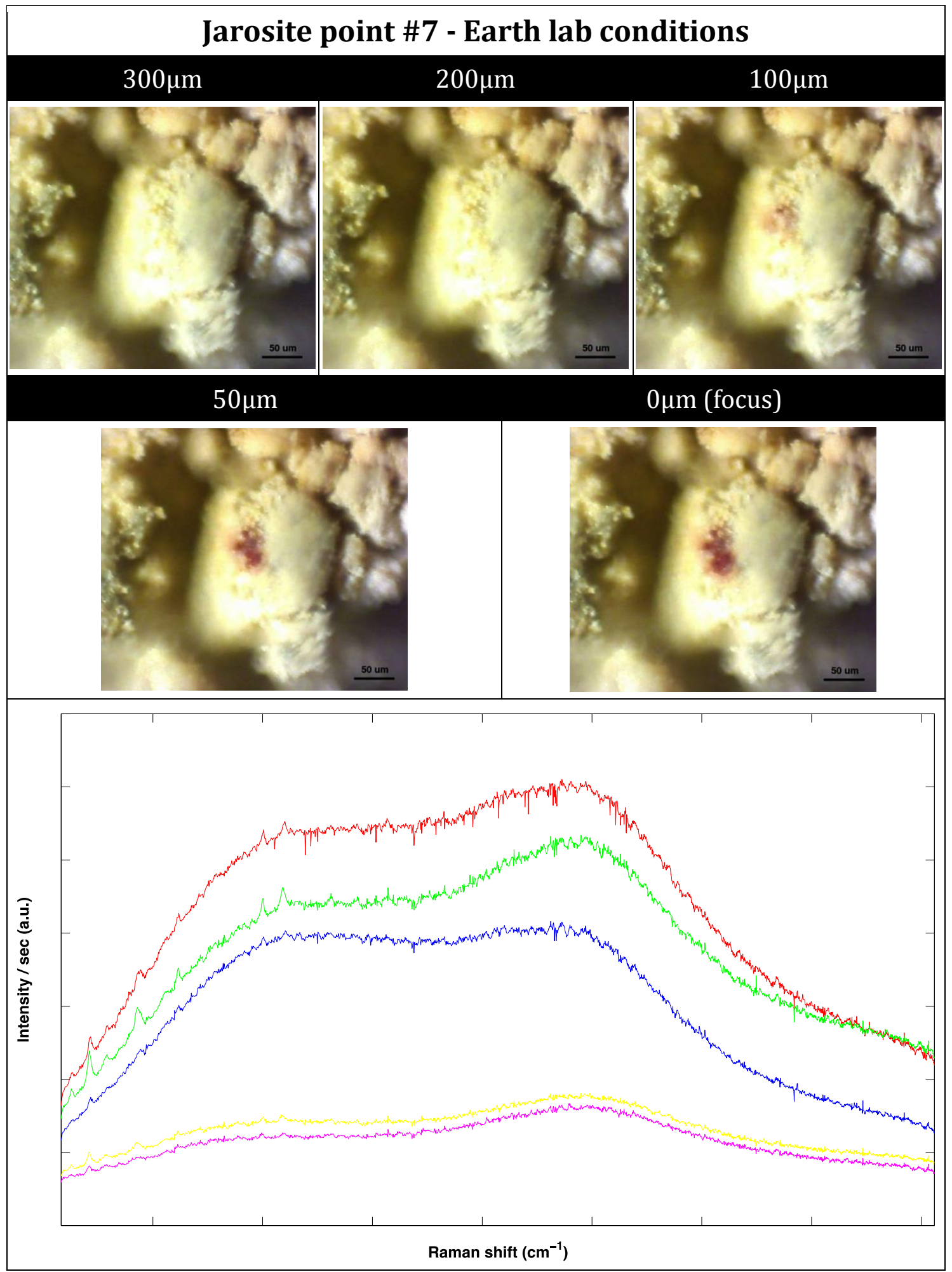

Table 5.43. - Height values from focus, images after exposure to laser and corresponding Raman spectra for the selected point \#7. Blue:300 $\mu \mathrm{m}$;

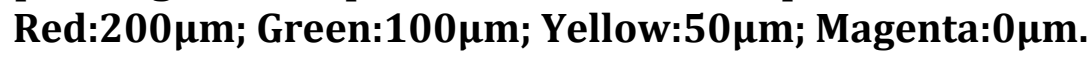



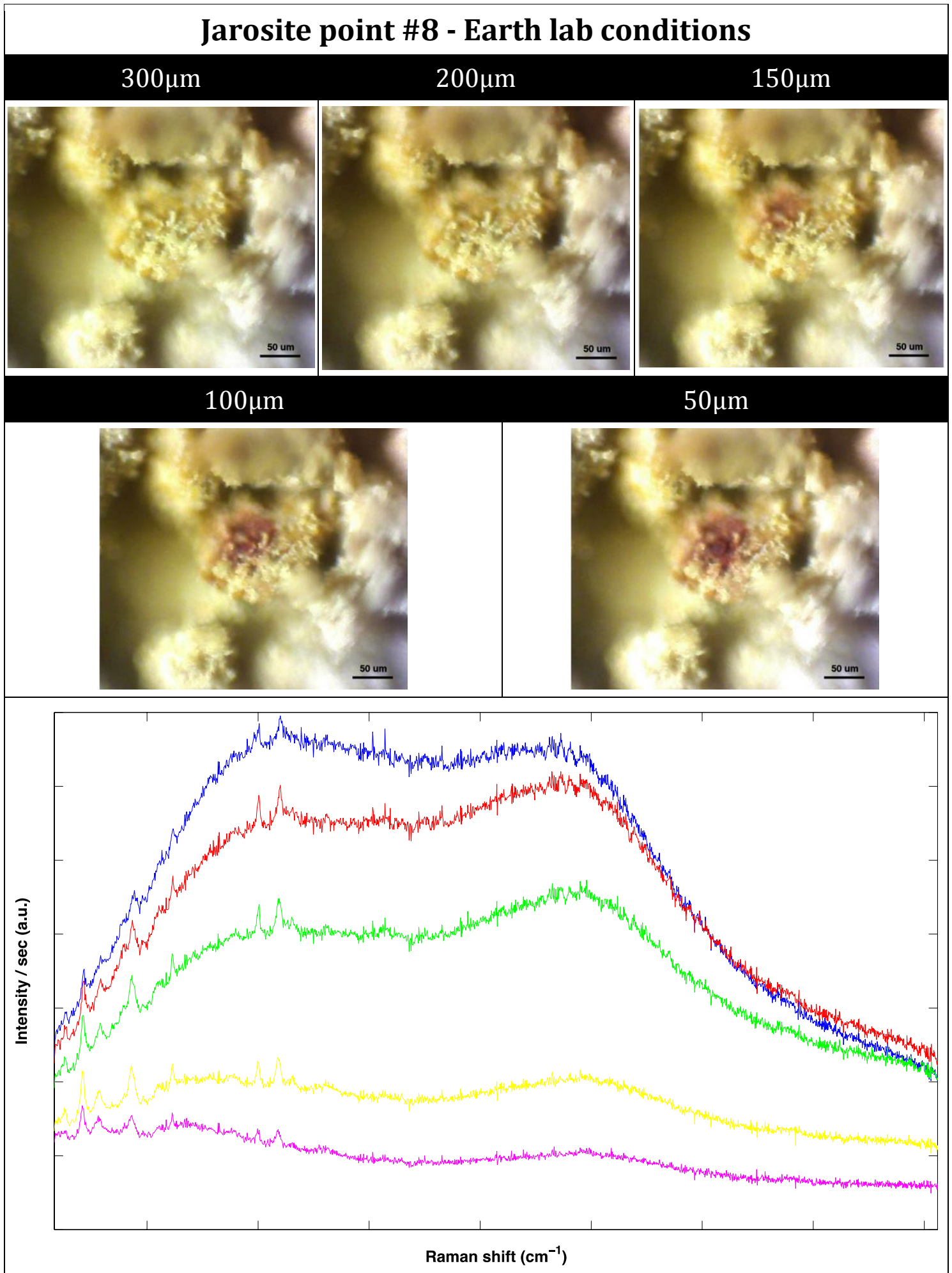

Table 5.44. - Height values from focus, images after exposure to laser and corresponding Raman spectra for the selected point \#8. Blue:300 $\mu \mathrm{m}$;

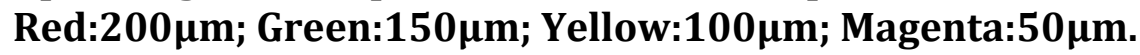




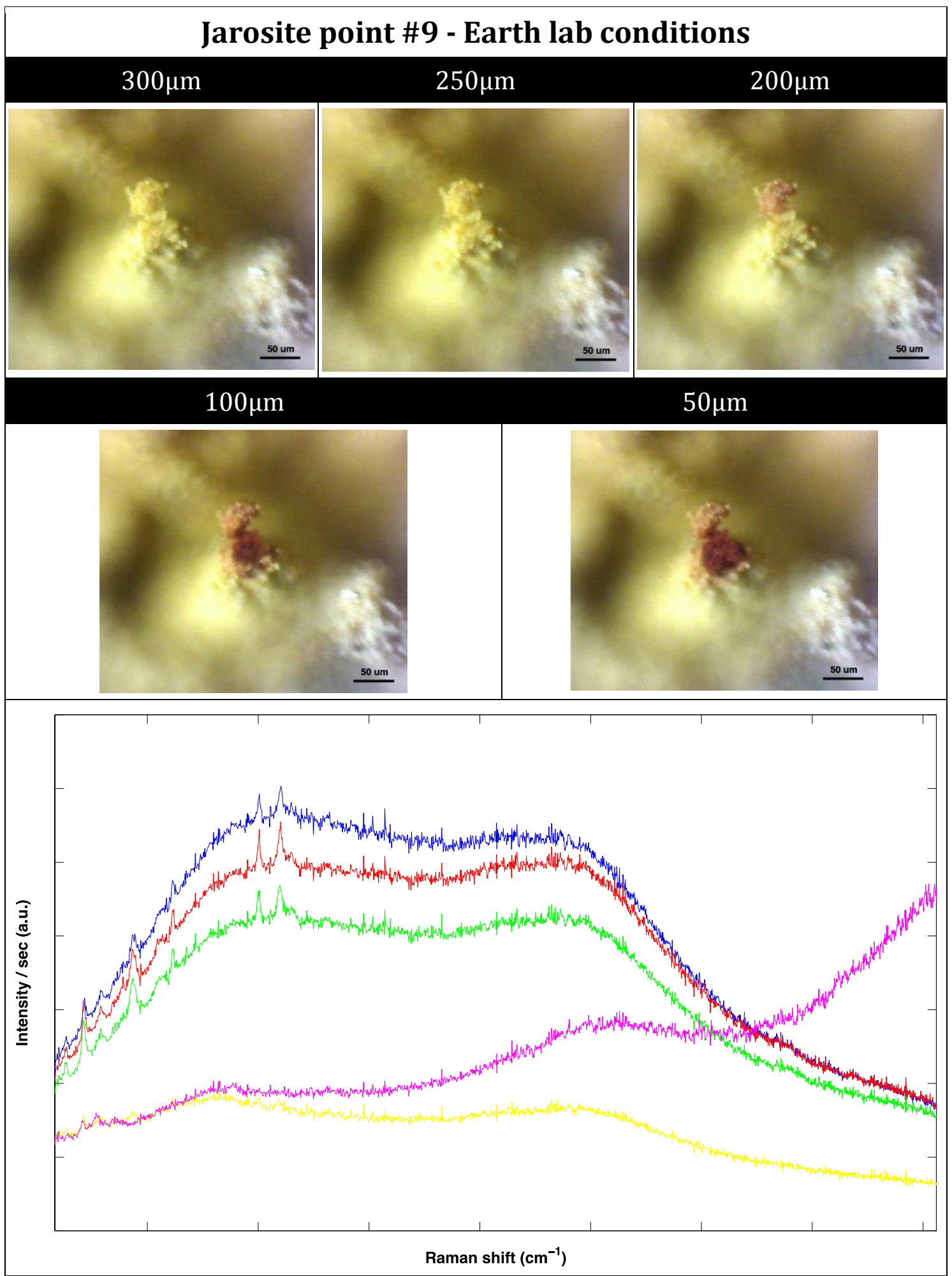

Table 5.45. - Height values from focus, images after exposure to laser and corresponding Raman spectra for the selected point \#9. Blue:300 $\mathrm{mm}$; Red:250 $\mu \mathrm{m}$; Green:200 $\mu \mathrm{m}$; Yellow:100 $\mu \mathrm{m}$; Magenta:50 $\mu \mathrm{m}$. 


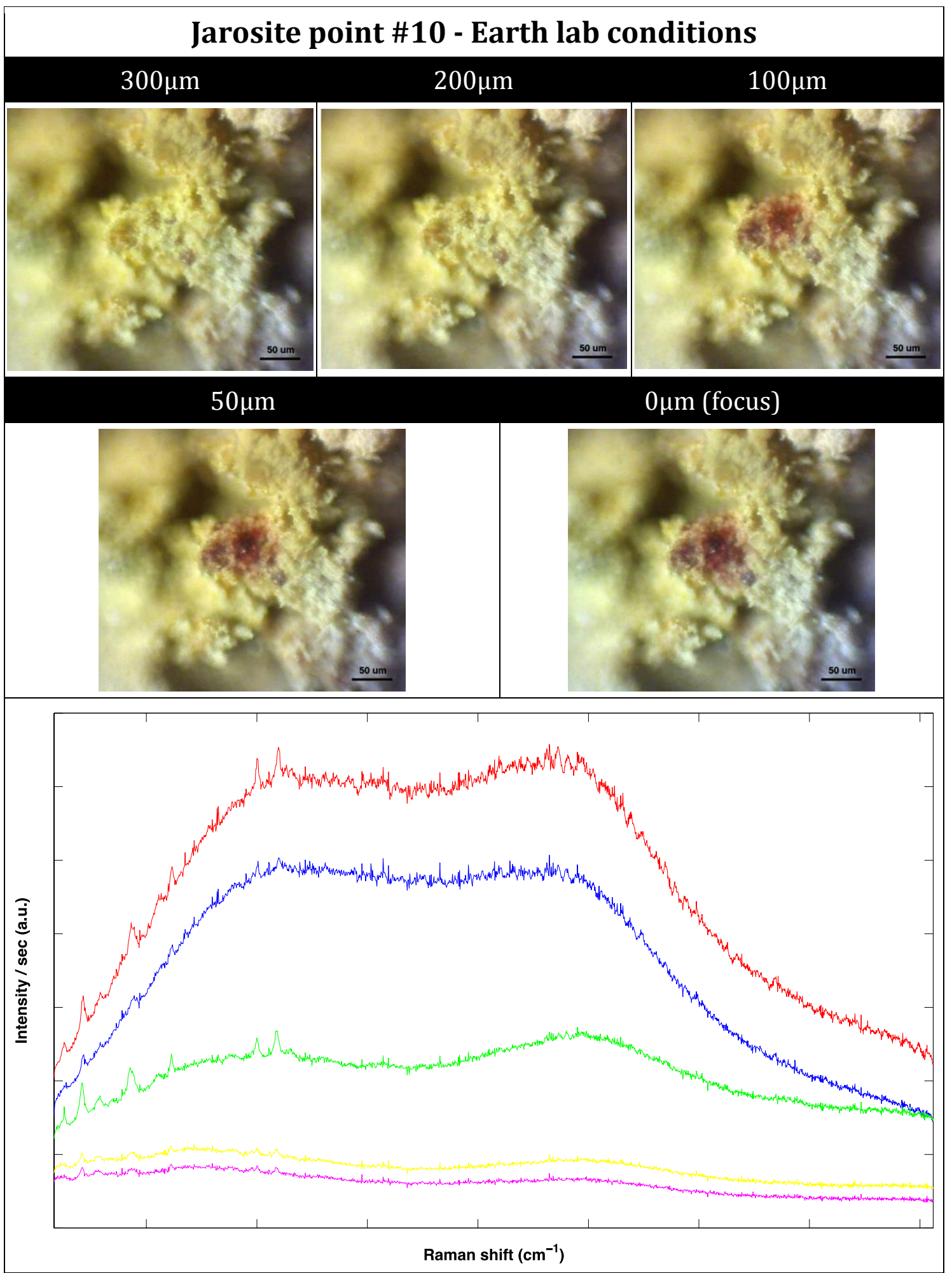

Table 5.46. - Height values from focus, images after exposure to laser and corresponding Raman spectra for the selected point \#10. Blue:300 $\mu \mathrm{m}$;

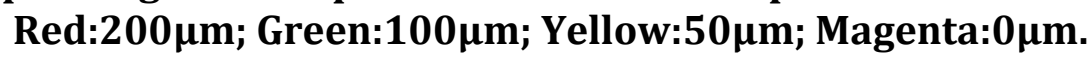


In this case, mineral grains appear to get damaged visually generally above $0.1 \mathrm{~kW} / \mathrm{cm}^{2}$, which is a higher value compared to RLS-ExoMars-like conditions.

Table 5.47 summarizes these results in terms of SNR values for $\mathrm{v} 1$ and for all cases.

\begin{tabular}{|c|c|c|c|c|}
\hline Point (\#) & $\begin{array}{l}\text { Height from } \\
\text { focus }(\mu \mathrm{m})\end{array}$ & $\begin{array}{c}\text { Spot size } \\
@ 13.5 \% \\
(\mu \mathrm{m})\end{array}$ & $\begin{array}{l}\text { Irradiance } \\
\left(\mathrm{kW} / \mathrm{cm}^{2}\right)\end{array}$ & $\operatorname{SNR}(\mathbf{v} 1)$ \\
\hline \multirow{5}{*}{6} & 300 & 155 & 0.071 & 5.5 \\
\hline & 200 & 111 & 0.140 & 5.0 \\
\hline & 100 & 70 & 0.357 & 5.0 \\
\hline & 50 & 57 & 0.522 & 4.0 \\
\hline & 0 & 53 & 0.605 & 1.9 \\
\hline \multirow{5}{*}{7} & 300 & 155 & 0.071 & 4.3 \\
\hline & 200 & 111 & 0.140 & 5.7 \\
\hline & 100 & 70 & 0.357 & 7.3 \\
\hline & 50 & 57 & 0.522 & 4.6 \\
\hline & 0 & 53 & 0.605 & 4.1 \\
\hline \multirow{5}{*}{8} & 300 & 155 & 0.071 & 6.2 \\
\hline & 200 & 111 & 0.140 & 9.1 \\
\hline & 150 & 89 & 0.220 & 9.7 \\
\hline & 100 & 70 & 0.357 & 7.9 \\
\hline & 50 & 57 & 0.522 & 5.5 \\
\hline \multirow{5}{*}{9} & 300 & 155 & 0.071 & 6.7 \\
\hline & 250 & 132 & 0.099 & 11.9 \\
\hline & 200 & 111 & 0.140 & 10.1 \\
\hline & 100 & 70 & 0.357 & 3.0 \\
\hline & 50 & 57 & 0.522 & 1.7 \\
\hline \multirow{5}{*}{10} & 300 & 155 & 0.071 & 5.7 \\
\hline & 200 & 111 & 0.140 & 8.2 \\
\hline & 100 & 70 & 0.357 & 9.4 \\
\hline & 50 & 57 & 0.522 & 5.9 \\
\hline & 0 & 53 & 0.605 & 6.7 \\
\hline
\end{tabular}

Table 5.47. - SNR values for the v1 peak of jarosite in all the Earth-like previous spectra. Height from focus, spot size at that height and irradiance is also presented in it. Cells marked in green correspond to the best SNR values for each point.

Attending to the optimum values of SNR and the heights at which the grains begin to degrade, there is once again a concordance. Once the grain is marked with the laser at certain height (irradiance), the spectral quality worsens hopelessly.

\subsubsection{Hematite}

The second sample in this line of analyses is hematite, in the specified grain size distribution. 


\subsection{Engineering data}

\subsection{Pressure and Temperature curves}

As it was explained for jarosite, there are no pressure and temperature curves as the environmental conditions are maintained after the performance of the power sweep experiment for this sample too. So, for the whole development of this analysis, the pressure is kept at a $\mathrm{CO}_{2}$ partial pressure of approximately $7.5 \mathrm{mbar}$ and the temperature at $-15^{\circ} \mathrm{C}$.

\subsection{Flattening performance}

Neither the RC nor the sample were moved after the power sweep test executed before this one. So, the line profiles obtained before are still valid.

\subsubsection{Results and Discussion}

Table 5.48 to Table 5.52 present the images and spectra corresponding to those irradiances around which the obtained SNR is maximum, and just before the tail at high RS begins to rise. 


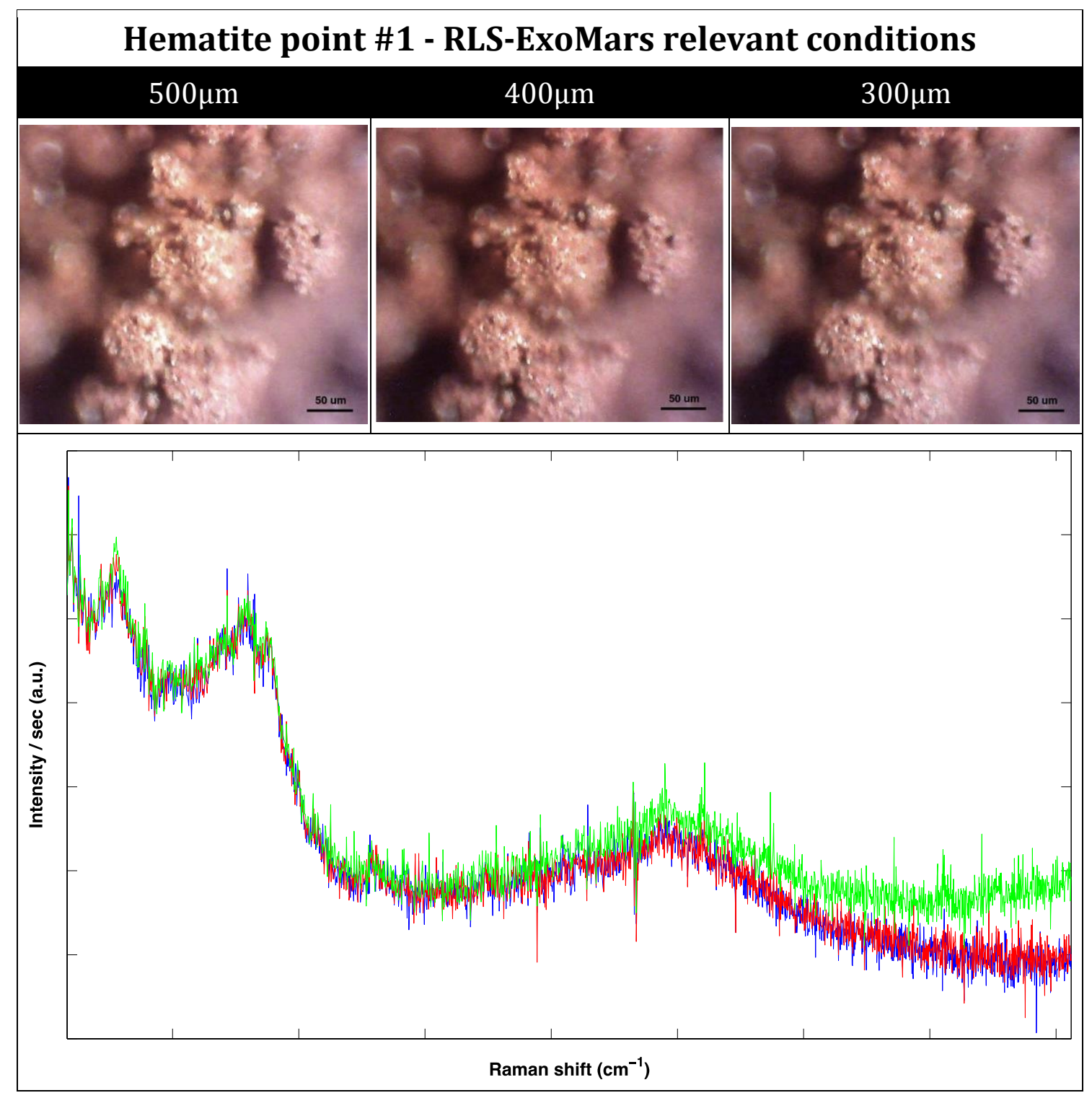

Table 5.48. - Height values from focus, images after exposure to laser and corresponding Raman spectra for the selected point \#1. Blue:500 $\mu \mathrm{m}$;

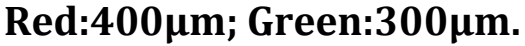




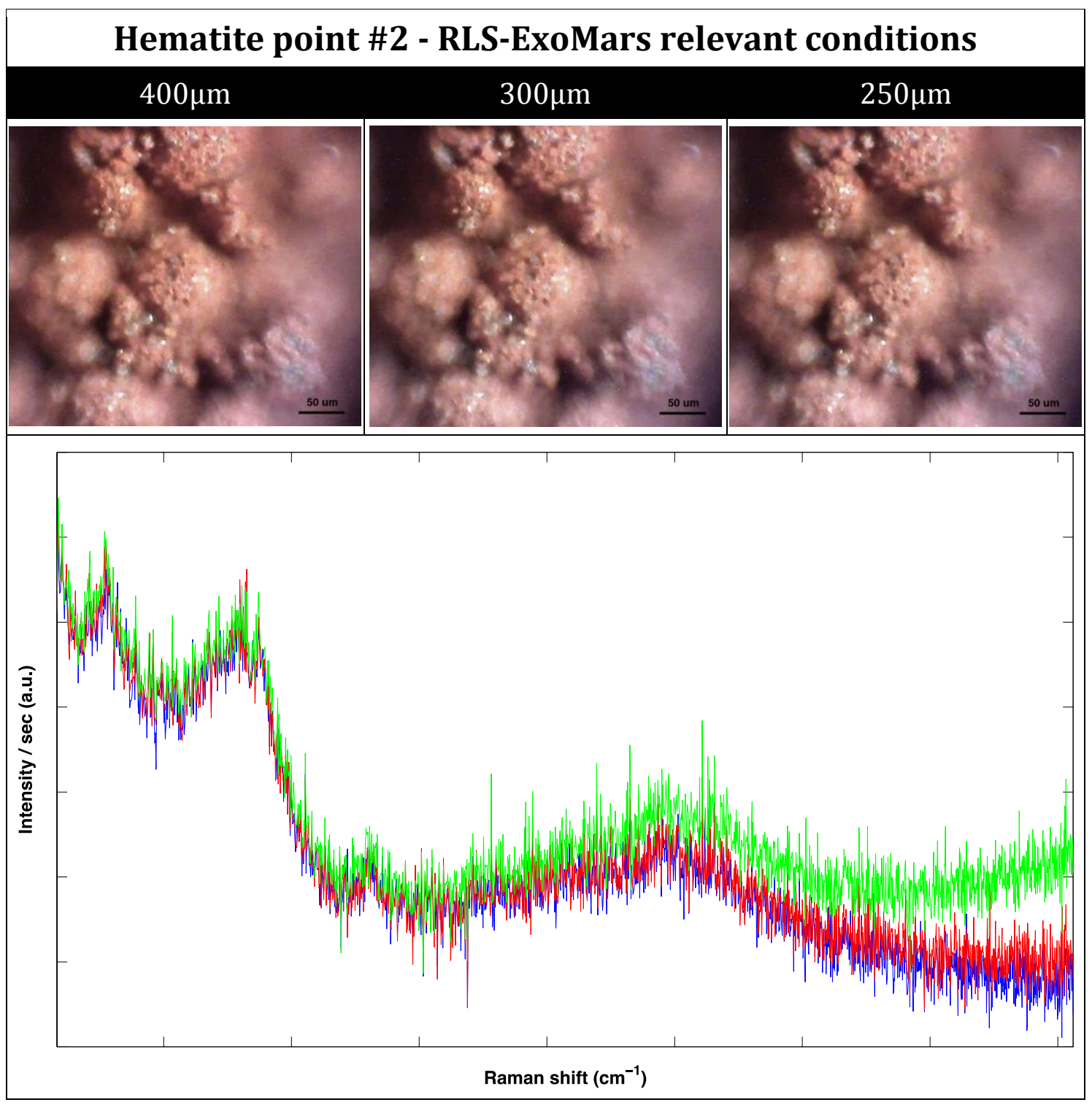

Table 5.49. - Height values from focus, images after exposure to laser and corresponding Raman spectra for the selected point \#2. Blue:400 $\mu \mathrm{m}$; Red:300 $\mu \mathrm{m}$; Green:250 $\mu \mathrm{m}$. 


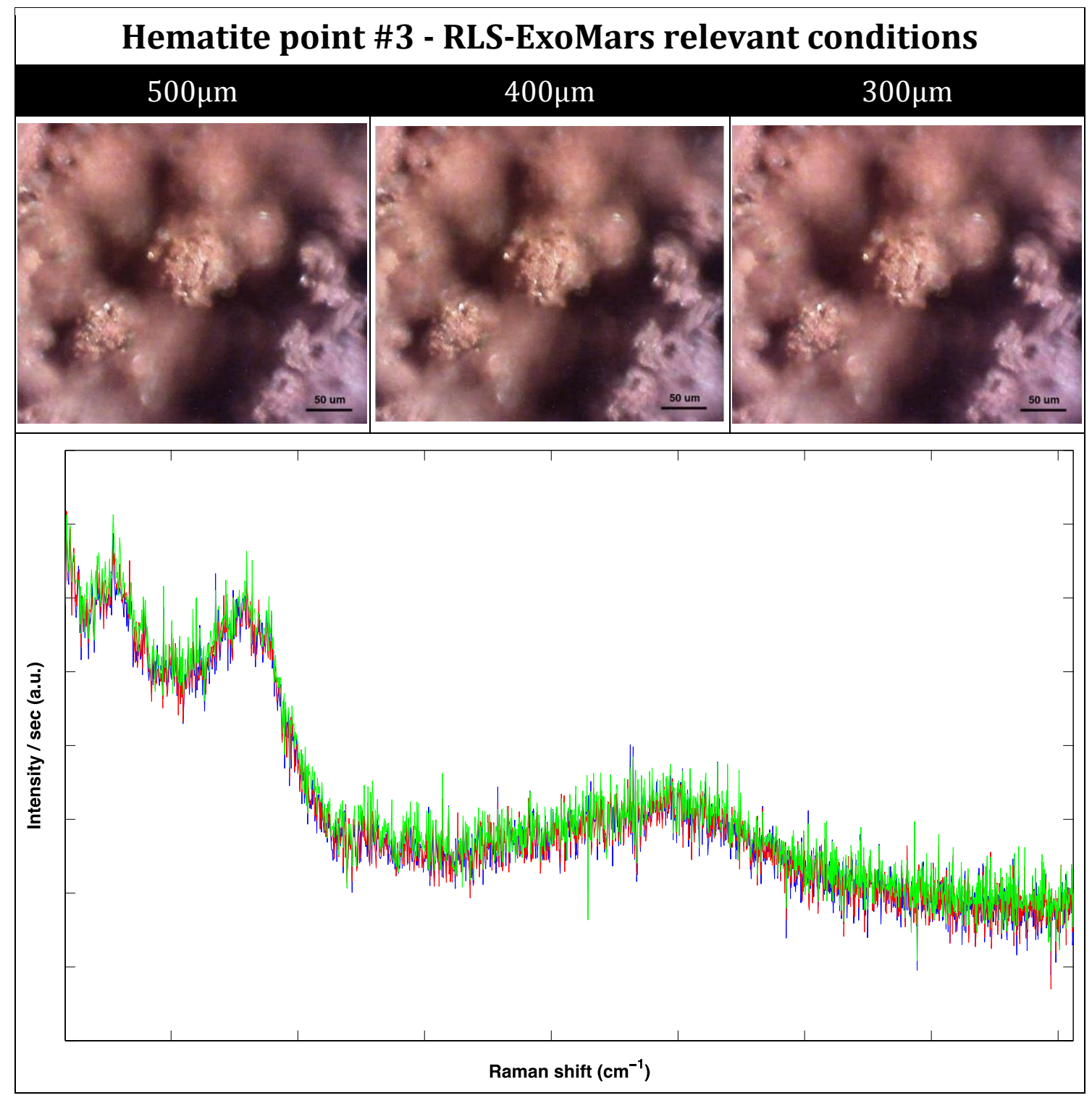

Table 5.50. - Height values from focus, images after exposure to laser and corresponding Raman spectra for the selected point \#3. Blue:500 $\mu \mathrm{m}$;

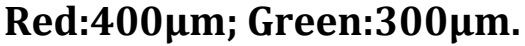




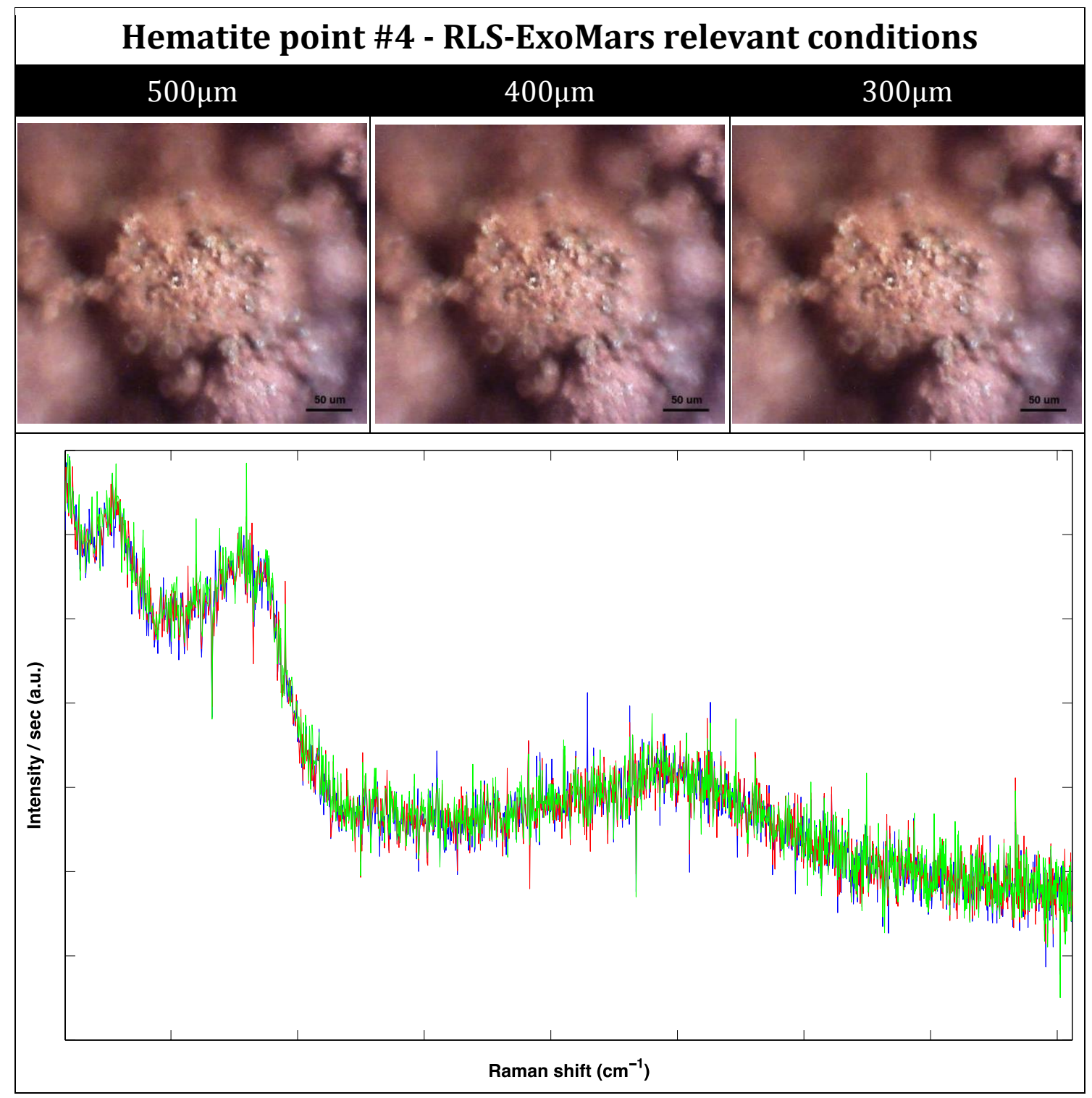

Table 5.51. - Height values from focus, images after exposure to laser and corresponding Raman spectra for the selected point \#4. Blue:500 $\mu \mathrm{m}$;

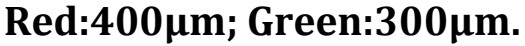




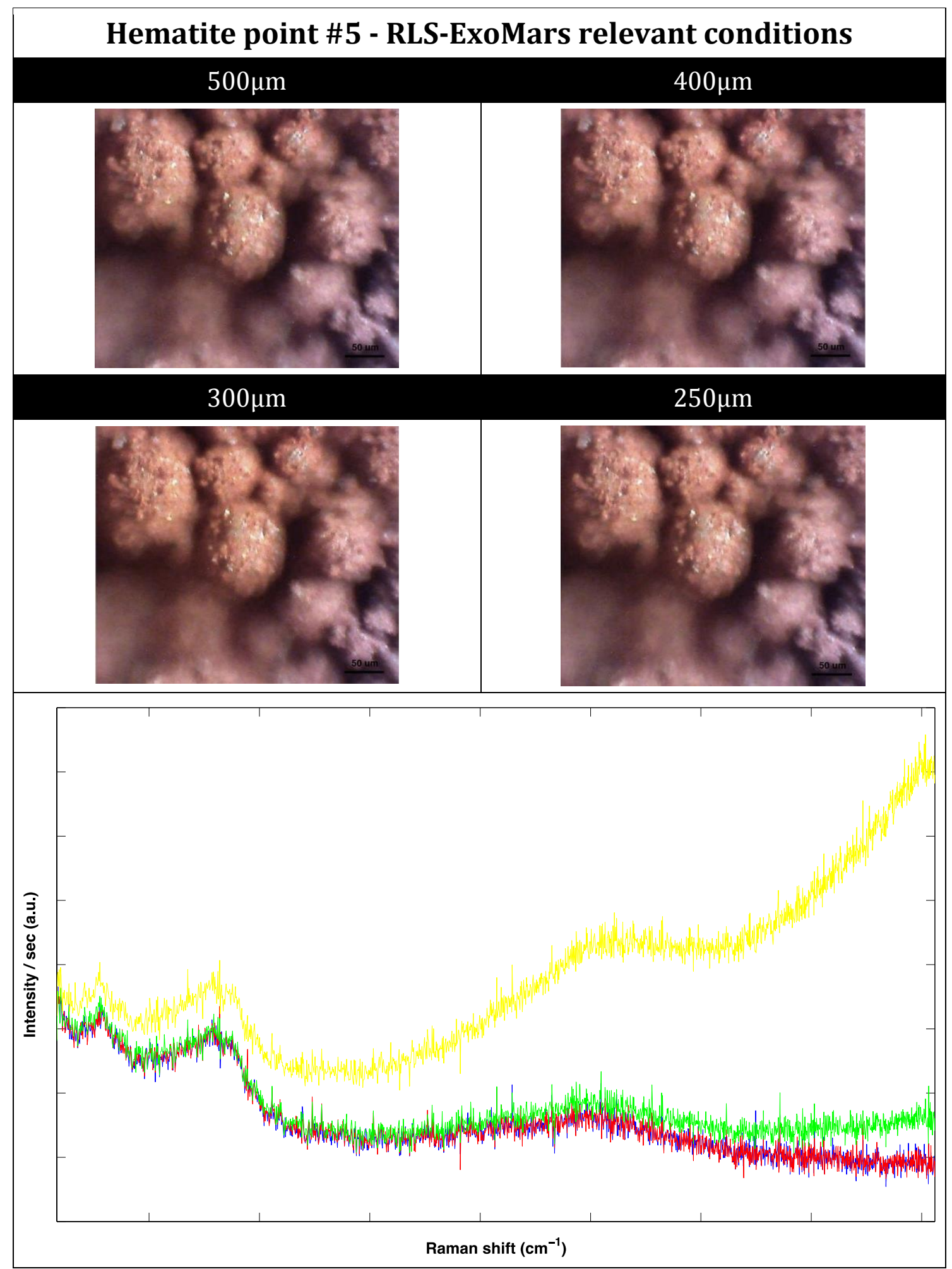

Table 5.52. - Height values from focus, images after exposure to laser and corresponding Raman spectra for the selected point \#5. Blue:500 $\mu \mathrm{m}$;

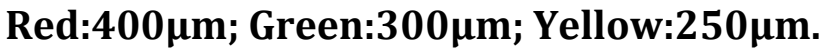


Being hematite, as previously noted, a poor Raman scatterer and reducing the irradiance to such low values, the spectra are consequently poor. Table 5.53 summarizes the results that previous tables present. In it, SNR values for the $405 \mathrm{~cm}^{-1}$ peak of hematite for the different acquired spectra are listed.

\begin{tabular}{|c|c|c|c|c|}
\hline \multirow{3}{*}{ Point (\#) } & $\begin{array}{c}\text { Height from } \\
\text { focus }(\mu \mathrm{m})\end{array}$ & $\begin{array}{c}\text { Spot size } \\
\text { @13.5\% } \\
(\mu \mathrm{m})\end{array}$ & $\begin{array}{c}\text { Irradiance } \\
\left(\mathrm{kW} / \mathrm{cm}^{2}\right)\end{array}$ & $\begin{array}{c}\text { SNR }\left(405 \mathrm{~cm}^{-1}\right. \\
\text { peak) }\end{array}$ \\
\hline \multirow{4}{*}{1} & 500 & 251 & 0.028 & 3.83 \\
\cline { 2 - 5 } & 400 & 200 & 0.043 & 5.57 \\
\hline \multirow{3}{*}{2} & 300 & 155 & 0.071 & 5.94 \\
\cline { 2 - 5 } & 400 & 200 & 0.043 & 4.45 \\
\cline { 2 - 5 } & 300 & 155 & 0.071 & 3.05 \\
\hline \multirow{3}{*}{4} & 250 & 132 & 0.099 & 4.71 \\
\cline { 2 - 5 } & 500 & 251 & 0.028 & 4.62 \\
\cline { 2 - 5 } & 400 & 200 & 0.043 & 4.23 \\
\cline { 2 - 5 } & 300 & 155 & 0.071 & 3.76 \\
\hline \multirow{3}{*}{5} & 500 & 251 & 0.028 & 3.28 \\
\cline { 2 - 5 } & 300 & 200 & 0.043 & 2.58 \\
\cline { 2 - 5 } & 300 & 155 & 0.071 & 2.82 \\
\cline { 2 - 5 } & 500 & 251 & 0.028 & 2.73 \\
\hline & 400 & 200 & 0.043 & 2.00 \\
\hline
\end{tabular}

Table 5.53. - SNR values for the $405 \mathrm{~cm}^{-1}$ peak of hematite in all the RLSExoMars-like previous spectra. Corresponding height from focus, spot size at that height and irradiance is also presented in it. Green cells show the best SNR values for each point.

Taking a look at the spectra from previous tables and to best SNR values in this table, not a large variation can be seen due to the poor spectral quality. Anyway, the highest values are obtained for irradiances below $0.1 \mathrm{~kW} / \mathrm{cm}^{2}$, or spot sizes greater than $130 \mu \mathrm{m}$.

In the next Table 5.54 to Table 5.57, show the corresponding hematite images and spectra for the same analysis performed under Earth lab conditions $\left(\mathrm{T}=20^{\circ} \mathrm{C}\right.$ and room pressure). 


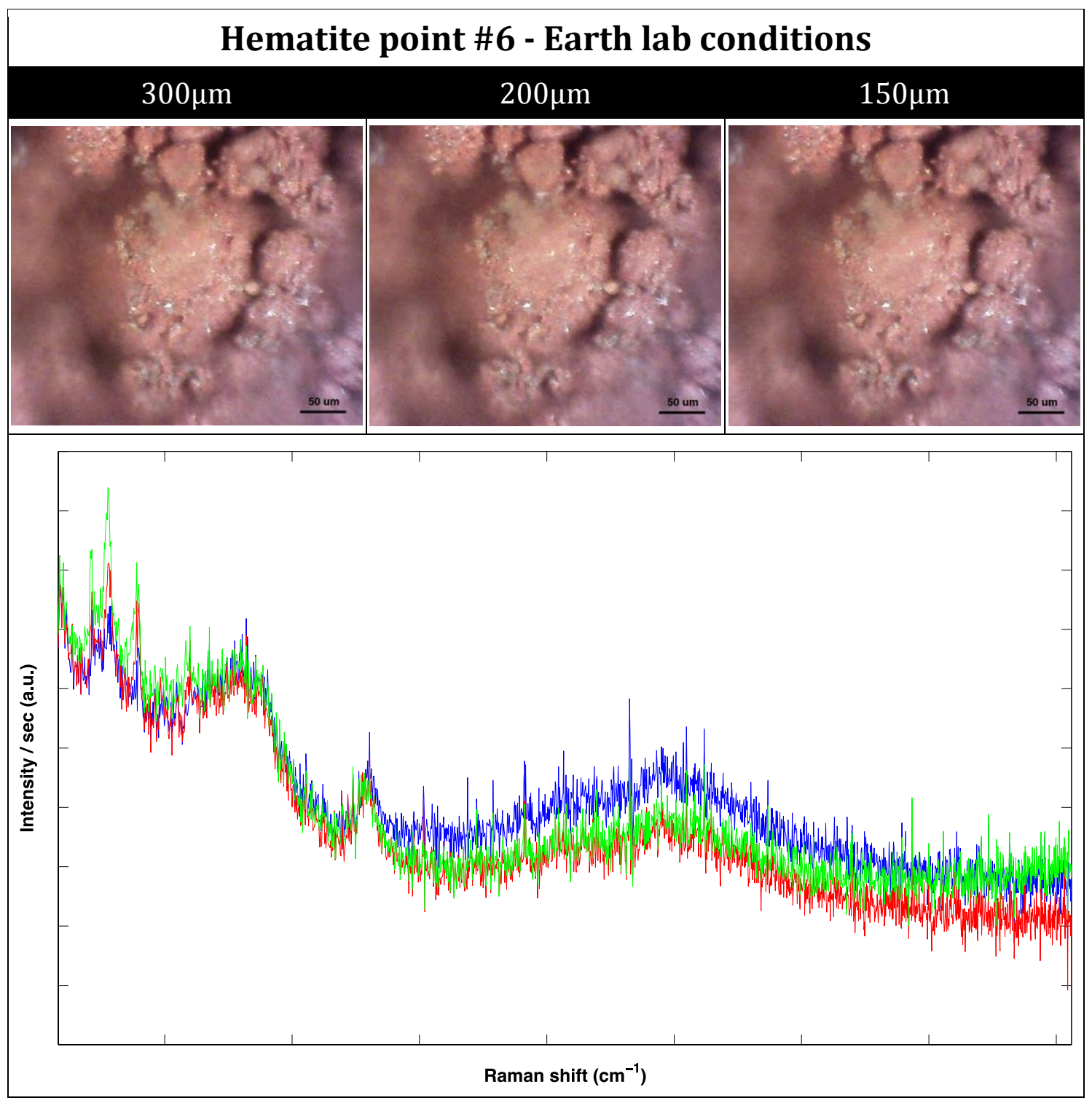

Table 5.54. - Height values from focus, images after exposure to laser and corresponding Raman spectra for the selected point \#6. Blue:300 $\mu \mathrm{m}$; Red:200 $\mu \mathrm{m}$; Green:150 $\mu \mathrm{m}$. 


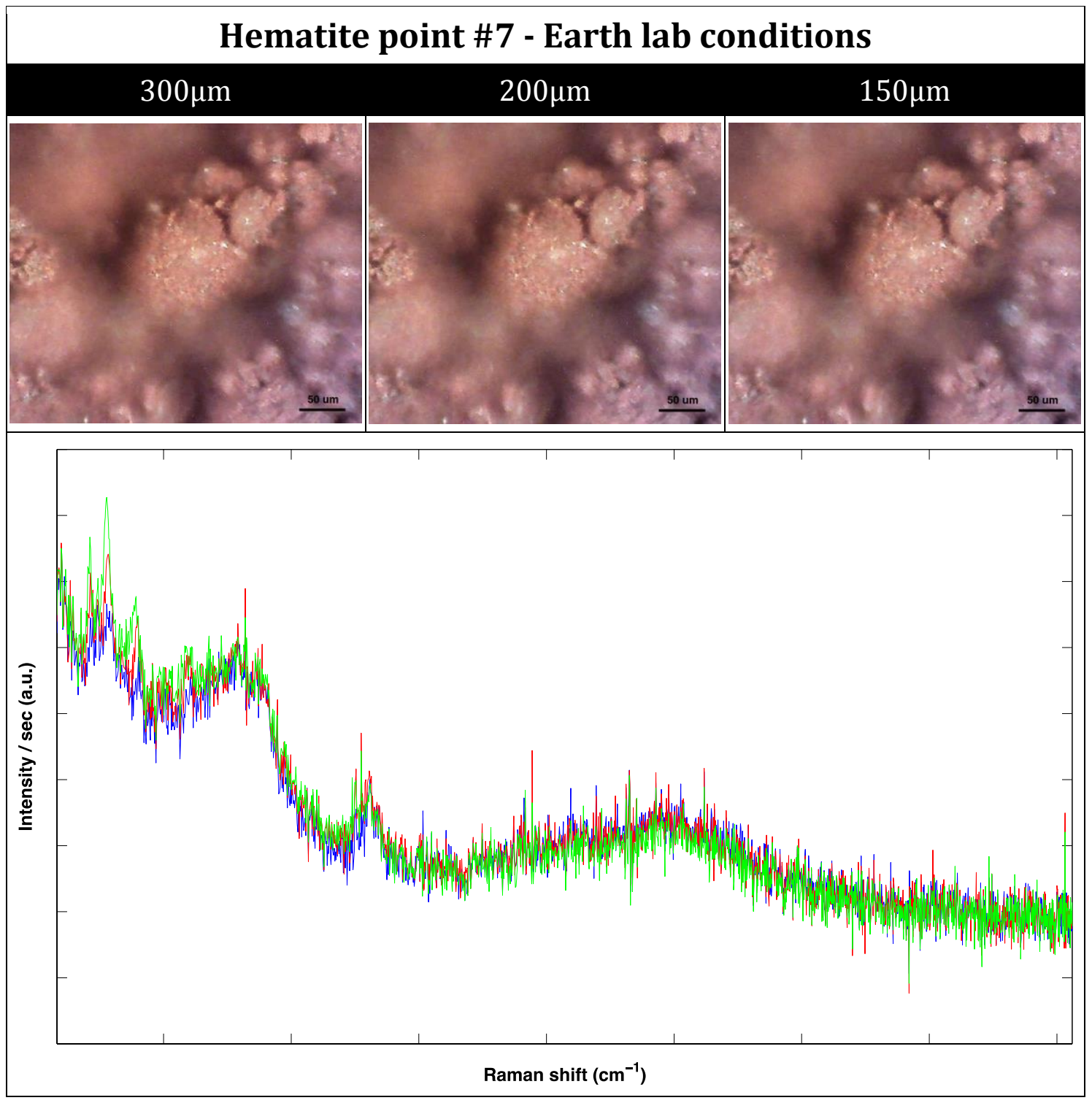

Table 5.55. - Height values from focus, images after exposure to laser and corresponding Raman spectra for the selected point \#7. Blue:300 $\mu \mathrm{m}$; Red:200 $\mu \mathrm{m}$; Green:150 $\mu \mathrm{m}$. 
Spectra from point \#8 got corrupted and the point has been discarded

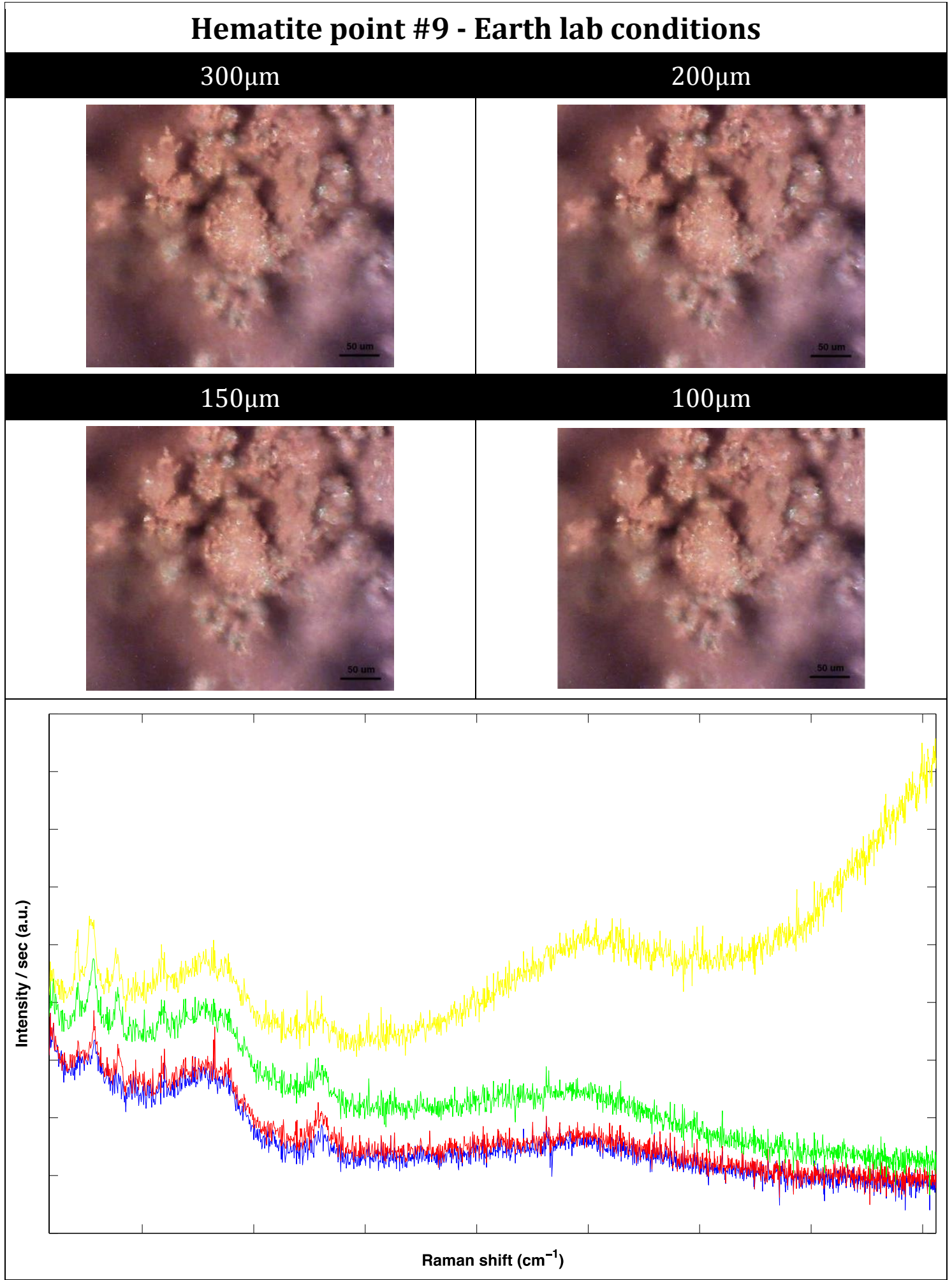

Table 5.56. - Height values from focus, images after exposure to laser and corresponding Raman spectra for the selected point \#9. Blue:300 $\mu \mathrm{m}$;

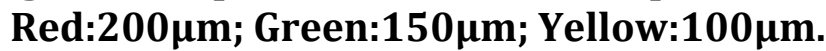




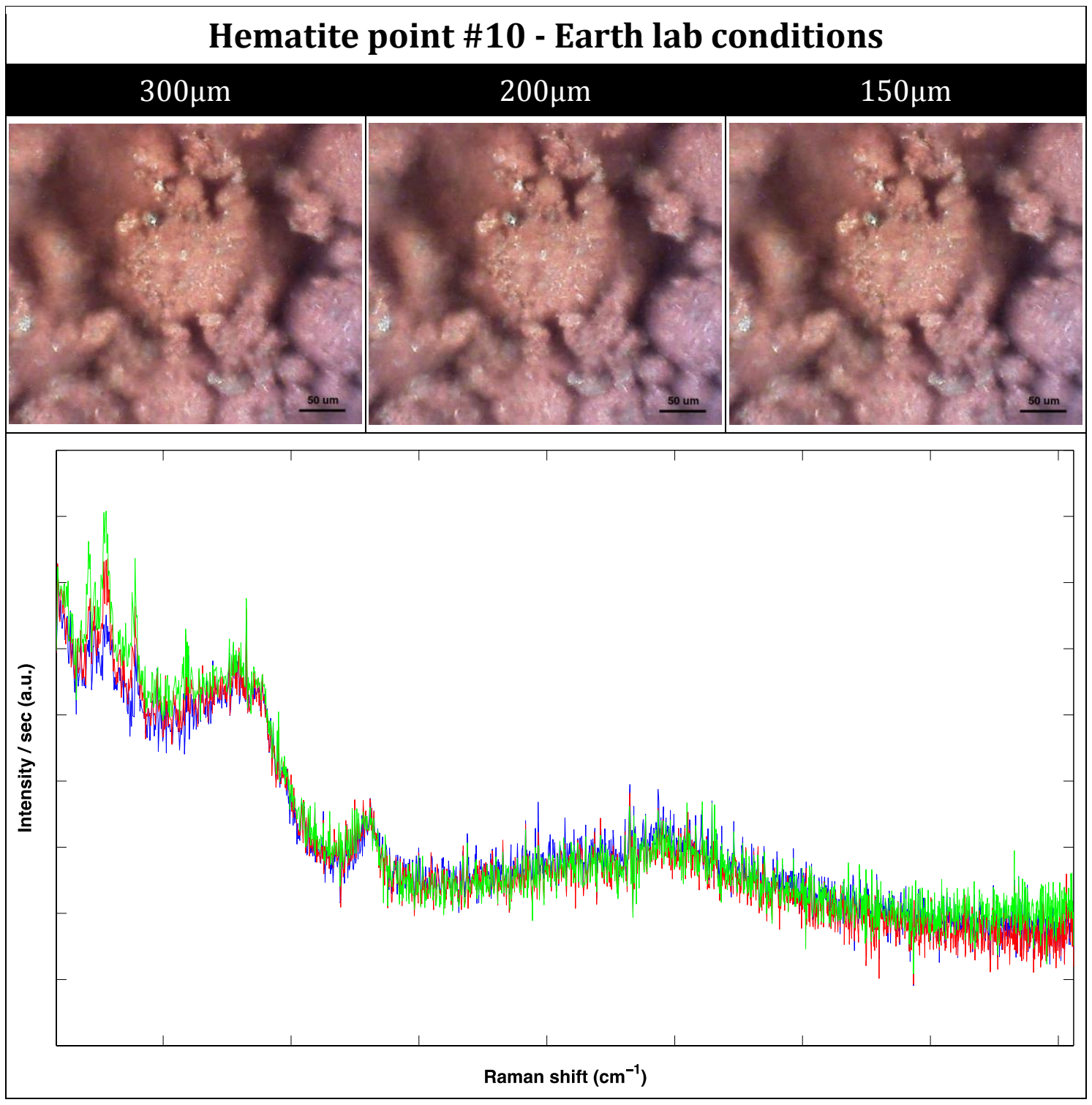

Table 5.57. - Height values from focus, images after exposure to laser and corresponding Raman spectra for the selected point \#10. Blue:300 $\mu \mathrm{m}$; Red:200 $\mu \mathrm{m}$; Green:150 $\mu \mathrm{m}$. 
Visually there are no differences among the mineral grains for this range of irradiances, as seen in previous experiments with hematite. Under these laboratory conditions, the spectral quality is better than for RLS-ExoMars-like conditions. Table 5.58 presents the SNR of the $405 \mathrm{~cm}^{-1}$ Raman peak at three heights from focus for four different points in the sample. At irradiances higher than those listed in it, the spectrum shows the behaviour of a damaged point and the spectral quality decreases.

\begin{tabular}{|c|c|c|c|c|}
\hline \multirow{3}{*}{ Point (\#) } & $\begin{array}{c}\text { Height from } \\
\text { focus }(\mu \mathrm{m})\end{array}$ & $\begin{array}{c}\text { Spot size } \\
\text { @13.5\% } \\
(\mu \mathrm{m})\end{array}$ & $\begin{array}{c}\text { Irradiance } \\
\left(\mathrm{kW} / \mathrm{cm}^{2}\right)\end{array}$ & $\begin{array}{c}\text { SNR }\left(405 \mathrm{~cm}^{-1}\right. \\
\text { peak) }\end{array}$ \\
\hline \multirow{3}{*}{6} & 300 & 155 & 0.071 & 4.47 \\
\cline { 2 - 5 } & 200 & 111 & 0.140 & 7.63 \\
\cline { 2 - 5 } & 150 & 89 & 0.220 & 10.22 \\
\cline { 2 - 5 } & 300 & 155 & 0.071 & 4.41 \\
\cline { 2 - 5 } & 200 & 111 & 0.140 & 5.86 \\
\hline \multirow{4}{*}{9} & 150 & 89 & 0.220 & 7.07 \\
\cline { 2 - 5 } & 300 & 155 & 0.071 & 4.27 \\
\cline { 2 - 5 } & 200 & 111 & 0.140 & 6.98 \\
\cline { 2 - 5 } & 150 & 89 & 0.220 & 6.29 \\
\hline \multirow{3}{*}{10} & 100 & 70 & 0.357 & 6.29 \\
\cline { 2 - 5 } & 300 & 155 & 0.071 & 8.49 \\
\hline
\end{tabular}

Table 5.58. - SNR values for the $405 \mathrm{~cm}^{-1}$ peak of hematite in all the Earth-like previous spectra. Associated height from focus, spot size at that height and irradiance is also presented in it. Cells in green mark the best SNR values for each point.

As shown in Table 5.58, the spot size that gives the best SNR in three out of four cases under these conditions is $\sim 90 \mu \mathrm{m}$, giving an irradiance of $0.22 \mathrm{~kW} / \mathrm{cm}^{2}$. This irradiance is higher than under RLS-ExoMars-like conditions, as it has been seen in the rest of tests with hematite and jarosite. This could be due to the fact that air convection plays an important role in heat dissipation. 


\subsection{Conclusions of the experiments}

In the first experiment a reduced group of relevant materials has been analysed inside the RLS-ExoMars Simulation Chamber and under several environmental conditions to assess how they affect the behaviour of the samples as reflected on their Raman spectra.

This experiment revealed that the stability of Alunite, Gypsum and Quartz is beyond these temperature and pressure changes. It cannot be said though for Jarosite, which presented evident shifts in frequency and the appearance of new bands at temperatures over zero.

The explanation we find for this behaviour implicates several agents: temperature of the sample, size of the grain, laser power and atmospheric pressure and composition. For a fixed size (volume) of the grain, the lower the temperature of the sample, the higher the irradiance needed to heat it to its thermal degradation point. The atmospheric pressure and it composition play also an important role in this thermal dissipation, as convection is an efficient means for heat transfer. At the point were the temperature of the sample is such that the irradiance can heat it beyond its thermal degradation point, transformation occurs. This point is also reached for $25^{\circ} \mathrm{C}$ and ambient pressure and gas composition, even though convection is present as it is observed with the database spectrum.

Attending to this first experiment, RLS-ExoMars mission would require of a special Raman spectral database to be able to distinguish minerals analysed at different environmental conditions, or at least under the currently defined for operation, and for thermolabile samples.

This experiment, opens the door to two future works: (1) extend this study for the rest of Mars relevant minerals already included in the Raman spectra database that the Unidad Asociada UVa-CSIC-CAB is building, and (2) go beyond the RLS-ExoMars temperatures and, taking advantage of the RLS-ExoMars Simulation Chamber preparation to go colder with its LN2 support, investigate the behaviour of the samples under the extreme Martian subsurface conditions. 
After the other two experiments, related to thermolability, what can be clearly concluded is that, for thermolabile samples such as Jarosite, the limits of irradiance defined for RLS-ExoMars are excessive in all cases: under RLS-ExoMars conditions and under Earth conditions. This irradiance value should be lowered if the RLS instrument is to analyse samples susceptible of being damaged by the interaction with focused laser light.

The lowering in irradiance can be done, as these experiments anticipate, by two means or a combination of them:

- Reducing the laser output power maintaining the spot size.

- Increasing the spot size of the Raman Optical Head leaving the laser power untouched.

The analysis in focus of mineral grains from thermolabile samples, under RLS-ExoMars environmental conditions, and sweeping in power, reveals a compromise irradiance for both jarosite and hematite. This irradiance, for marking the sample just enough to get good SNR figures seems to be $0.127 \mathrm{~kW} / \mathrm{cm}^{2}$, which corresponds to a laser power at the output of the optical path of $2.84 \mathrm{~mW}$. That is $20 \%$ the current minimum irradiance defined for RLS-ExoMars. Lower irradiances may not be enough to acquire good spectra in poor Raman scatterers and higher irradiances could damage the sample or displace it from the focus, not getting spectra at all.

The other performed experiment, the one that studies the effect on the Raman spectra produced by the variation of the laser spot size (simulated here as a defocusing by moving the optics away from the focus), establishes $0.1 \mathrm{~kW} / \mathrm{cm}^{2}$ as the value of maximum irradiance for the RLS-ExoMars conditions. This value translates into a $\sim 130 \mu \mathrm{m}$ diameter for the spot, working at the power that, with a $50 \mu \mathrm{m}$ spot and in focus corresponds to $0.6 \mathrm{~kW} / \mathrm{cm}^{2}$. This value is 2.6 times the diameter of the current spot. It is important to highlight that the SNR values presented in Table 5.41, Table 5.47, Table 5.54 and Table 5.58 are not real in terms of what would be expected using higher spot sizes. This is, again, because the optical system being used is confocal. And, as the experiment has been defined, defocusing the excitation to get lower irradiance means also defocusing the Raman 
collection. So, with the calculated spot in focus, it is expected to get better SNR figures. Using higher diameters means, additionally, to illuminate a larger area on the sample and, in heterogeneous samples, to maybe getting information from several mineral species, which could be an advantage.

Another possibility, more functional and less instrumental, would be to detect in some manner that the grain being analysed has been damaged. In that case, the procedure might be to move to an adjacent point and take another spectrum at an upper height (taking advantage of the autofocus movement capability), thus reducing the irradiance. Although this solution has the direct advantage of not having to modify the current scientific requirements and the instrument, it presents some drawbacks:

- A new algorithm for detecting damaged samples has to be developed, tested and implemented.

- High irradiances could result in phase transformations on the minerals being irradiated, getting spectra from the transformation and not from the original substance.

- Transforming samples might mean not detecting valuable and perhaps unique (in our line of analysis) mineral or biological species.

- The time for getting spectra of a sample increases with the number of thermolabile species that are found along the line of analysis.

- Acquiring spectra at a height out of focus, even inside the DOF, degrades their SNR.

- If the mineral grain in focus were displaced by the effect of the laser (as it has been seen during the performance of the experiments presented in this document), the damage detection algorithm would not detect a damaged sample, as there is no sample. The result would just be a Raman spectrum with no peaks at all.

Thermolability and thermal effects are delicate issues. They depend on factors, many of which are beyond our control, as is the case for the properties of the sample: surface-to-volume ratio, degree of crystallization of the mineral grain, absorption coefficient, etc. The only constraints we can apply to our instrument are 
those instrumental and methodological. Not only the power or the spot size (or both) have to be carefully selected, but the time a thermolabile sample is being irradiated contributes to its degrading, even at safe irradiances. This means that, fluorescence-reducing algorithms, which contemplate irradiance on the sample as the way to reduce this effect and prior to taking Raman spectra, can be harmful to the sample in terms of this degrading.

An example on how different a sample can behave due to the effect of laser incidence is seen in jarosite and hematite. Jarosite dehydrates and oxidises to get transformed into other chemical species, losing its original spectral information.

Meanwhile, Hematite is a stable mineral at high temperature. After being heated by the laser and having shown the black body radiation tail, the original spectral features can be recovered lowering the irradiance (see the preliminary results obtained at LINES with Gossan under Earth lab conditions in Figure 5.11), as long as the grain is not damaged. Probably, this would not have happened if Goethite or Magnetite had been exposed to laser radiation (Shebanova \& Lazor, 2003). Hematite is then an example of reversible laser heating.

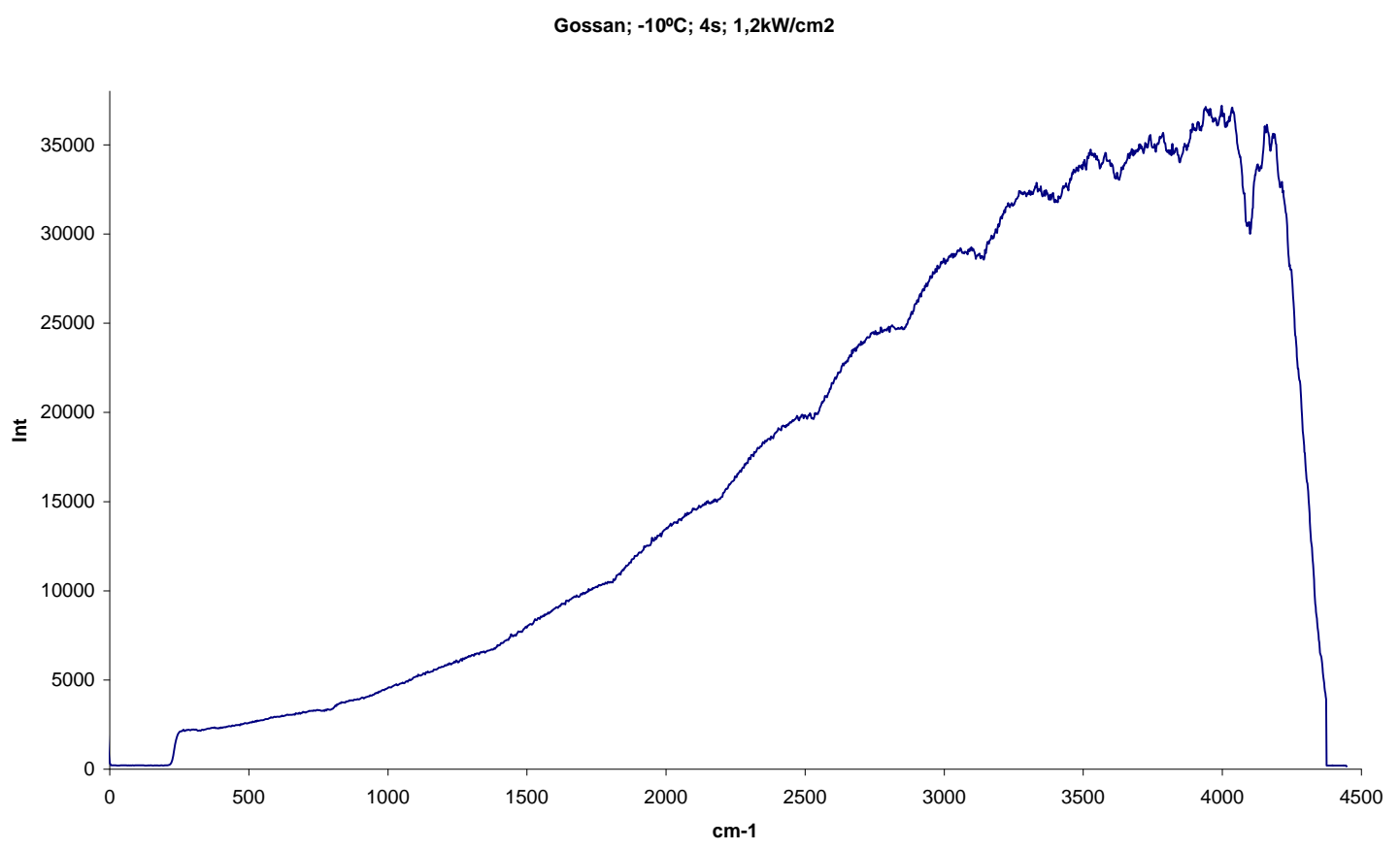


Gossan; -10ำ; 60s; 0,2kW/cm2

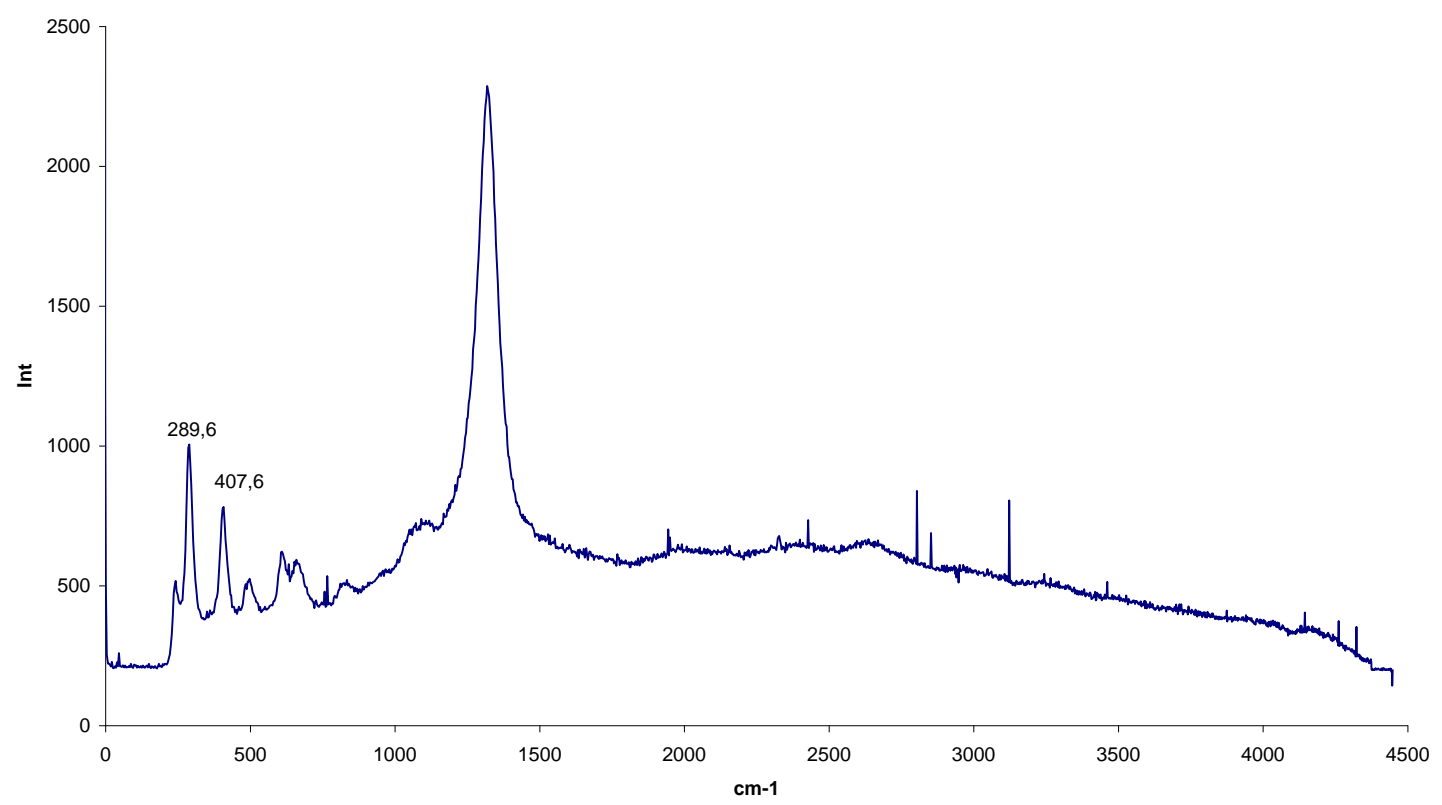

Figure 5.11. - Spectra of Gossan (Hematite + Goethite) for $1.2 \mathrm{~kW} / \mathrm{cm}^{2}$ (top) and $0.2 \mathrm{~kW} / \mathrm{cm}^{2}$ (bottom). The latter was acquired later. The Raman peaks are recovered, as the grain has not been irreversibly damaged with the high irradiance. (Credit: LINES, INTA) 


\section{Chapter 6}

\section{Conclusions}

This chapter presents the conclusions of this $\mathrm{PhD}$ thesis. It also presents ideas for future works to be developed with the RLS-ExoMars Simulation Chamber in order to continuing pushing the limits of knowledge in regards to Raman spectroscopy of materials, both organic and inorganic that could sometime be found on the surface or subsurface of our planetary neighbour. 



\subsection{Conclusions of this PhD thesis}

These are the conclusions that summarize the work presented in this $\mathrm{PhD}$ thesis manuscript:

1. The RLS-ExoMars Simulation Chamber in combination with the RLS-ExoMars Science Simulator have been designed and constructed as tools for the support science for the RLS-ExoMars instrument.

The RLS-ExoMars Simulation Chamber is able of cooling the sample to temperatures of operation $\left(<-5^{\circ} \mathrm{C}\right)$ and below. It is also able to recreate in its interior the atmospheric pressures and temperatures that the samples will experience inside the ExoMars rover, in the Analytical Laboratory Drawer (ALD). It has a motorized blade that can flatten the powdered sample in the refillable container as the ExoMars preparation for analysis.

The RLS-ExoMars Science Simulator is capable of positioning the Raman Optical Head along and accross the container inside the chamber, and access the sample at 100x visually and spectroscopically through the optical window installed in the top cover of the chamber for that purpose.

The environmental conditions and the positioning of the Raman Head are established and monitored through a custom-designed software for PC. The flattening process is manually commanded through a PCB and embedded software custom-designed for this project.

2. From the comparative of Raman analyses under Martian and Terrestrial conditions for assessing the necessity of creating a mission-specific Raman spectral database, it can be concluded that for thermolabile samples such as Jarosite this database is of paramount importance. Thermolabile samples behave differently under different temperature and pressure conditions because of laser-induced thermal heating, which can trigger transformations. A new database containing all these possibilities for different grain size distributions will be a powerful tool for data exploitation in the context of the ExoMars mission.

3. With respect to these thermal transformations, defining irradiance values on samples and more importantly on thermolabile samples is the most important task to avoid thermal effects that could not allow obtaining correct and high 
quality Raman spectra of the minerals under study. It has been concluded that, for the new grain size distribution scenario in which the lower tail extends to $50 \mu \mathrm{m}$ and beyond, irradiance has to be certainly reduced from the current values. A compromise between laser power and overall Raman performance of the mission has to be found in order to being able to analyse all kinds of samples, specially those that justify and will prove the objectives of the mission. The ways of doing this are various: laser power reduction, laser spot enlargement, algorithms for damage detection, etc.

\subsection{Future work}

Having this tool available for testing materials related to the RLS instrument under ExoMars conditions, countless are the experiments that can be imagined to continue doing science, defining the instrument and its operation, for preparing this mission and many more to come.

The most urgent task to do in relation to ExoMars science is the compilation of the RLS-ExoMars database of spectra against which to compare the received ones once the rover sends them back from Mars.

New tests to be performed include how the instrument will deal with fluorescent samples. This lies in the field of operation mode of the instrument under RLS-ExoMars relevant conditions, a work that begun with the precursor of the RLS-ExoMars Science Simulator.

In parallel it is time to begin the integration of the final version of the RLS instrument being built at INTA with a new RLS-ExoMars Simulation Chamber based in the one here presented. This will help the RLS scientists to constrain the scientific requirements for the instrument such as establishing the limits of irradiance, assessing the SNR that can be obtained and fixing the acquisition parameters that will optimize the time and resources of the mission to return the best analytical material the science can have to reach valuable conclusions regarding life on Mars.

The RLS-ExoMars Simulation Chamber is prepared (changing a few elements) for the simulation of other Martian environments. Lowering the 
temperature of the samples to values near $-90^{\circ} \mathrm{C}$ and heating from there can help us understand the transformation processes that the samples suffer from the subsurface of Mars to the interior of ExoMars's ALD.

And going further, the environments on the surface of other planetary bodies of the Solar System could also be simulated, foreseeing future exploration missions.

But this tool cannot only serve science in the field of geochemistry or even planetary exploration. This tool can be used to analyse minerals and living organisms but also natural and synthetic materials for industy and study their behaviour under low pressure and low temperature conditions. 



\section{Annex A}

\section{PC Software}

Annex A shows a screen capture of the software created for controlling the pressure and temperature systems of the RLS-ExoMars Simulation Chamber as well as the XYZ movements of the RLS-ExoMars Science Simulator. It also stores temperature and pressure logs, and an operations log with every action that is taken. 

Figure A.1 presents a screen capture of the software designed for the control of the temperature and pressure systems of the RLS-ExoMars Simulation Chamber as well as the XYZ movements of the RLS-ExoMars Science Simulator.

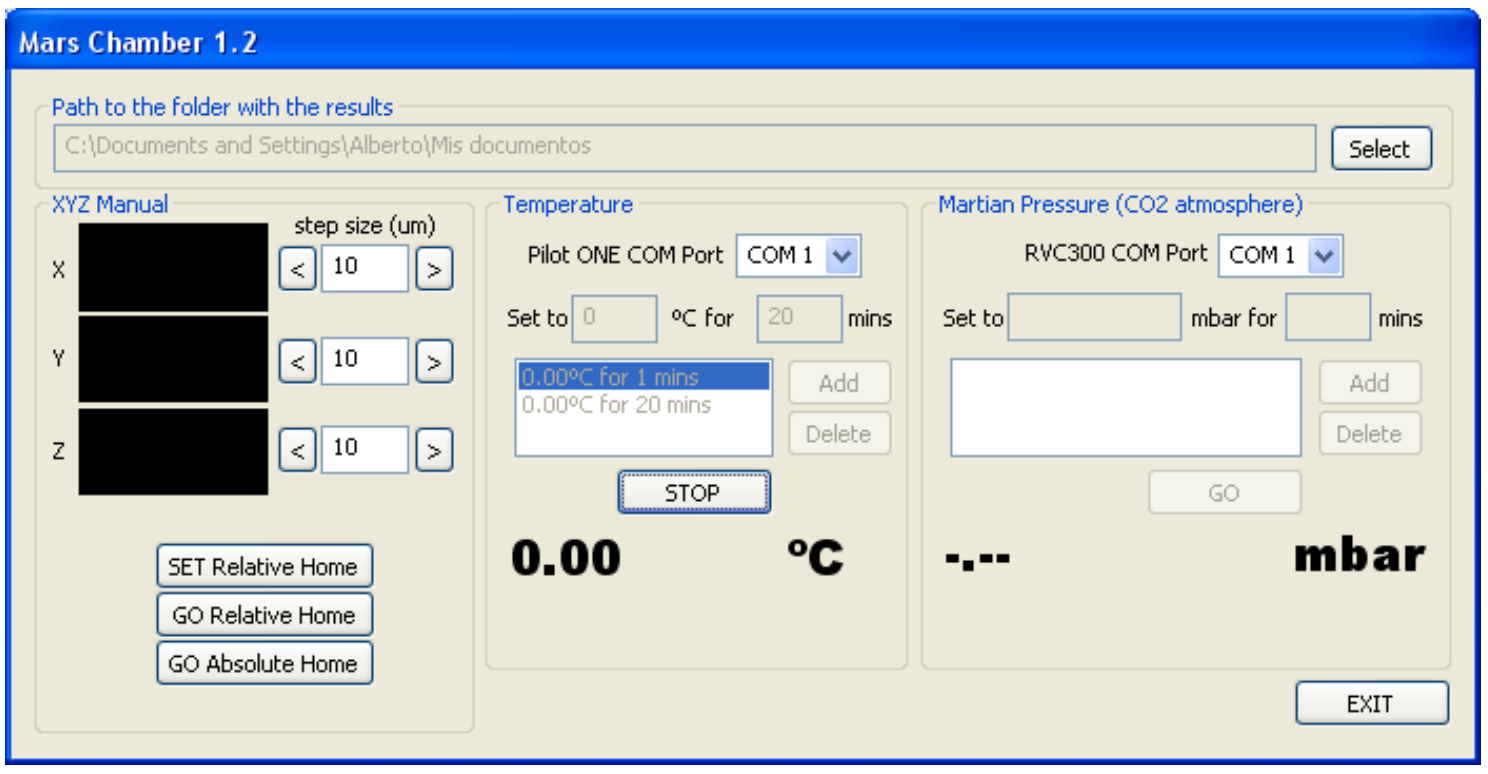

Figure A.1. - Screen capture of the PC Software for controlling the $R L S$ ExoMars Simulation Chamber and the RLS-ExoMars Science Simulator

This figure presents several panels with different functionalities that are going to be explained:

At the top of the window, there is a panel showing the path to the folder where the temperature, pressure and operations logs will be stored. This folder can be selected by means of the button on the right of the panel, labeled "Select".

Below that panel three more lay: the leftmost controls the XYZ movement, the one at the centre controls temperature and the rightmost, pressure.

The movement controller presents three displays and three controls for each of the directions of movement. The controls are comprised of: (1) a text field were the step size can be specified, and (2) two buttons to move the specified step size forward or backward. At the left of the controls the numerical displays show the current position of the optical system of the RLS-ExoMars Science Simulator expressed in millimeters.

The lower part of the movement controller has three larger buttons to set and go to relative and absolute home. Absolute HOME is the origin of coordinates 
$(0,0,0)$ for the three motors that drive the three linear stages. Relative home can be set wherever the operator desires by pressing the "SET Relative Home" button. The position shown at the displays will be set as the relative home. The operator can move to that relative home any time by just pressing the "GO Relative Home" button. These two buttons are useful to establish a starting point for measurements, for example, at the beginning of the central line of the refillable container. After performing the analyses for that line on the sample it is easy to go back to the pre-established start position.

The panel at its right allows opening a USB connection to the Pilot ONE controller attached to the Huber Ministat refrigeration unit. Through this port the software is able to establish temperature setpoints to command the Ministat the desired temperatures at the refillable container. These temperatures will be automatically reached aided with the feedback provided by the PT100 temperature sensor inside the refillable container. For obvious reasons all controls are disabled until the communication with the Pilot ONE has been successfully established. The software allows creating a scheduled temperature control creating a list of temperatures to be reached during a certain amount of time. That is the reason for the existence of the "Add" and "Remove" buttons. By filling the text fields between "Set to" and "mins" the operator specifies the desired temperature to be reached and during how much time it must be maintained this order. The "Add" button adds the order to the table as a new entry. This can be done as many times as needed, giving flexibility to the experimental conditions that can be defined. The "Delete" button after selecting one of the entries in the table deletes it and moves the next orders upwards. Once the temperature conditions have been programmed through entries in the table, the process can be started at any time pushing the "GO" button. No new orders can be added neither deleted once the process has started. This action also marks the starting time for the temperature log to begin storing temperature values (in ${ }^{\circ} \mathrm{C}$ ) at the rate of one per second in a .txt file. In the case all the entries of the table have been executed, the temperature of the last entry will be maintained until the operator decides to stop the temperature control by pressing the "STOP" button. In such case, the temperature setpoint is set to $20^{\circ} \mathrm{C}$ and the system tends to that value. 
The rightmost panel, in charge of the pressure control does exactly the same as its predecessor does for temperature. In this case the communication is established with the RVC300 Pressure Controller which, controlling the electroactuated gas flow valve and with the feedback from the Pirani pressure sensor, adjusts and maintains the desired pressures inside the RLS-ExoMars Simulation Chamber. The $\mathrm{CO}_{2}$ gas is continuously flowing into the chamber as the pumping station is continuously pumping out the gas from it. Leaving the gas flow valve closed the final pressure inside the chamber would reach a pressure in the order of the microbars. This way of proceeding ensures that the gas pressure inside the chamber is achieved with only the desired gas and the contribution from "terrestrial gasses" that could make it into the chamber due to leakage is limited and maintained below known limits. Pushing the "GO" button begins writting the pressure log as well as establishing the pressure steps (in mbars) defined in the table above it.

Finally, the "EXIT" button stops the temperature and pressure logs and control, disconnects the Pilot ONE and the RVC300 units and exits the PC application closing its window. 



\section{Annex B}

\section{Stepper motor controller}

This Annex presents a deeper look on the stepper motor controller, the electronic circuit and software that controls the rotation of the motor and thus the movement of the flattening tool inside the RLS-ExoMars Simulation Chamber. 



\section{Stepper motor pinout}

Figure B.1 shows both the image of the pinout of the motor and the table with the states to be assigned to each of the pins to get the motor moving through the different steps.

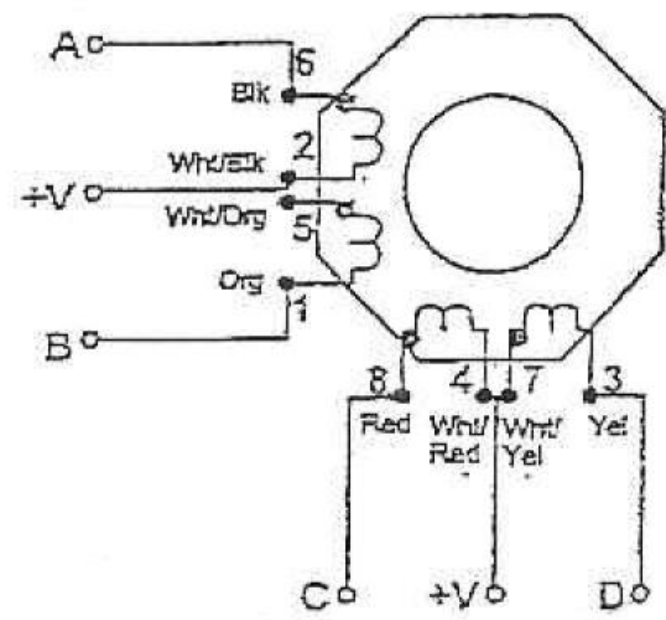

a

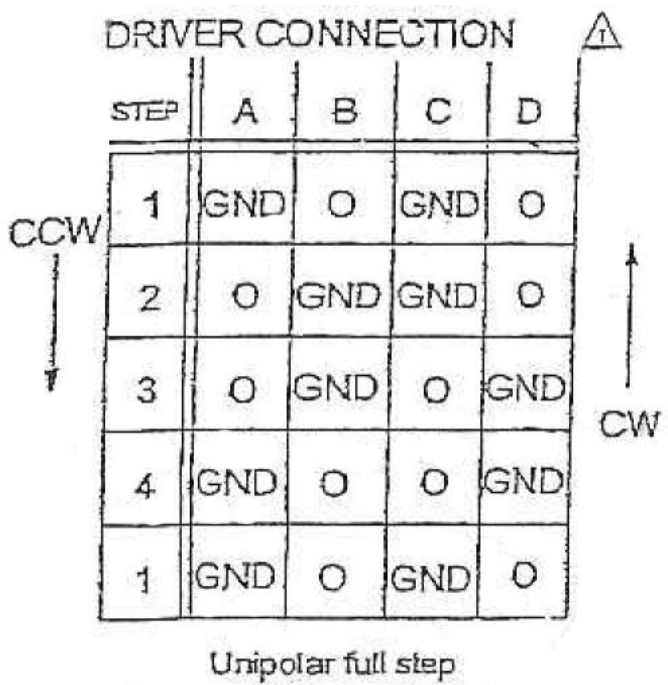

$\mathrm{b}$

Figure B.1. - Stepper motor pinout (a) and table of states (b). (Credit: MDC Vacuum Products, LLC)

The controlling electronics will determine the voltages applied to the A, B, C and $\mathrm{D}$ pins of the motor to move it among steps and turn it in one or the other direction.

\section{Circuit Board and Electronic Components}

Figure B.2 reproduces again Figure 3.21 but adding aiding text and shapes encapsulating the different groups of electronic components performing a coordinated function. 


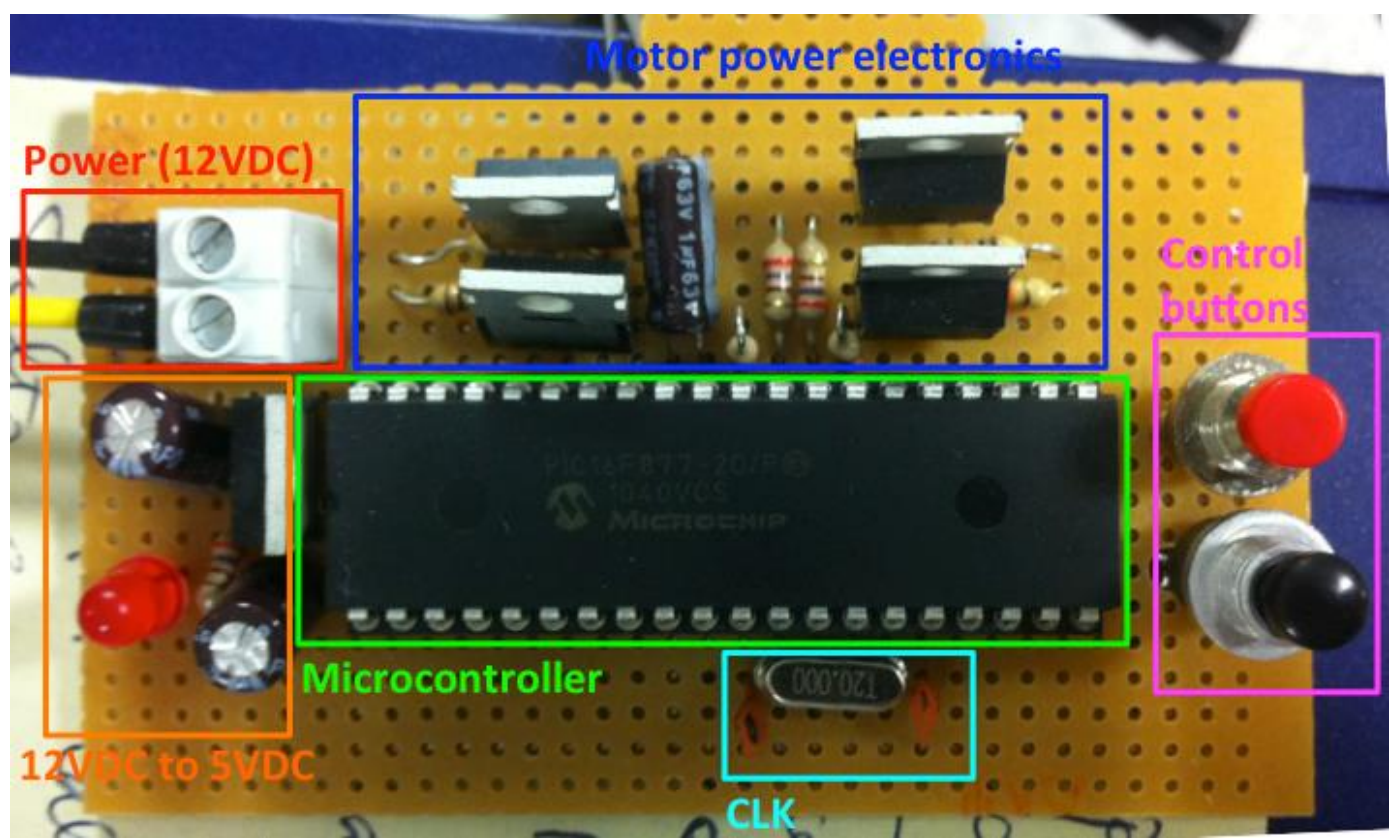

Figure B.2. - Circuit board with functional groups of components

The red box labelled 'Power (12VDC)' is the connection port for the power of the circuit board. The black cable is GND and the Yellow cable is +12VDC.

Below this box there is an orange one with the label '12VDC to 5VDC'. This stage has a voltage regulator for converting the 12VDC input to 5VDC voltage to power the microcontroller. The 12VDC are used to feed the motor. This box also contains a red LED to visually tell when the circuit board is powered up.

To the right of this group of electronics there is a green box containing the 'Microcontroller'. This is a PIC16F877 microcontroller by Microchip. This is the brain of the circuit board, which contains the instructions and controls the inputs and outputs of the board.

Next to it there is a cyan box ('CLK') with a $20 \mathrm{MHz}$ crystal connected to the microcontroller. This is a paramount element; it is like the heartbeat at which to execute the instructions.

The dark blue box above the microcontroller contains all the 'Motor power electronics' for commanding the motor steps from the microcontroller. The adequate timing of signals makes the motor turn and the flattening tool flatten. 
The rightmost box of the image, the magenta one labelled 'Control buttons', has two push buttons. These are the user interfaces to the system. The user will push and hold (1) the black button to move the flattening tool forward for flattening the sample and (2) the red button to move the flattening tool backwards to the beginning of the $\mathrm{RC}$, the origin position.

\section{Program inside the microcontroller}

The following lines present the program that is running from the moment the Circuit Board is powered on and how it reacts to the action of pressing the interface buttons on it.

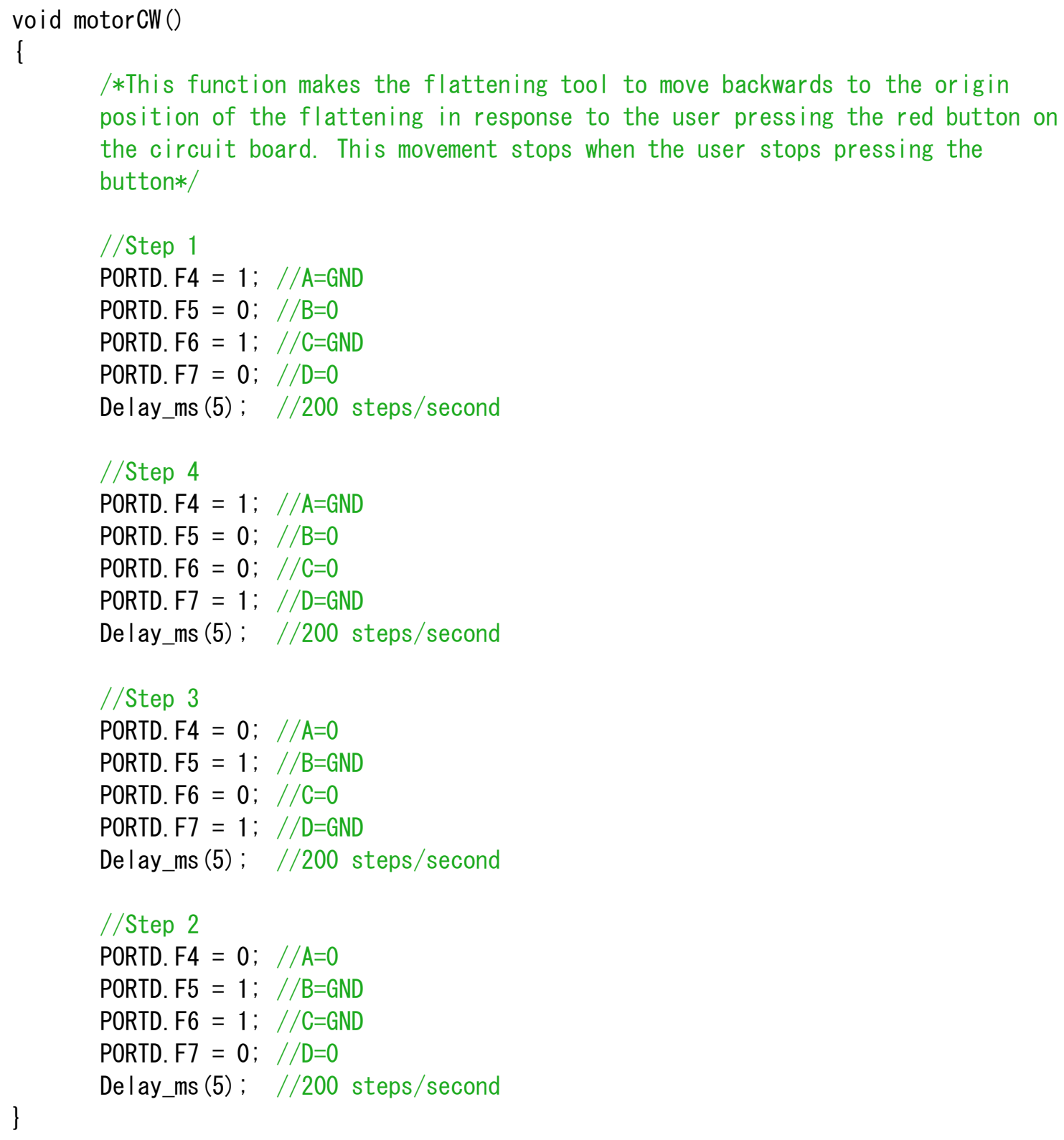




\section{Alejandro Catalá Espí}

void motor(CW ()

\{

$/ *$ This function makes the flattening tool to move forward to the end position of the flattening in response to the user pressing the black button on the circuit board. This movement stops when the user stops pressing the button*/

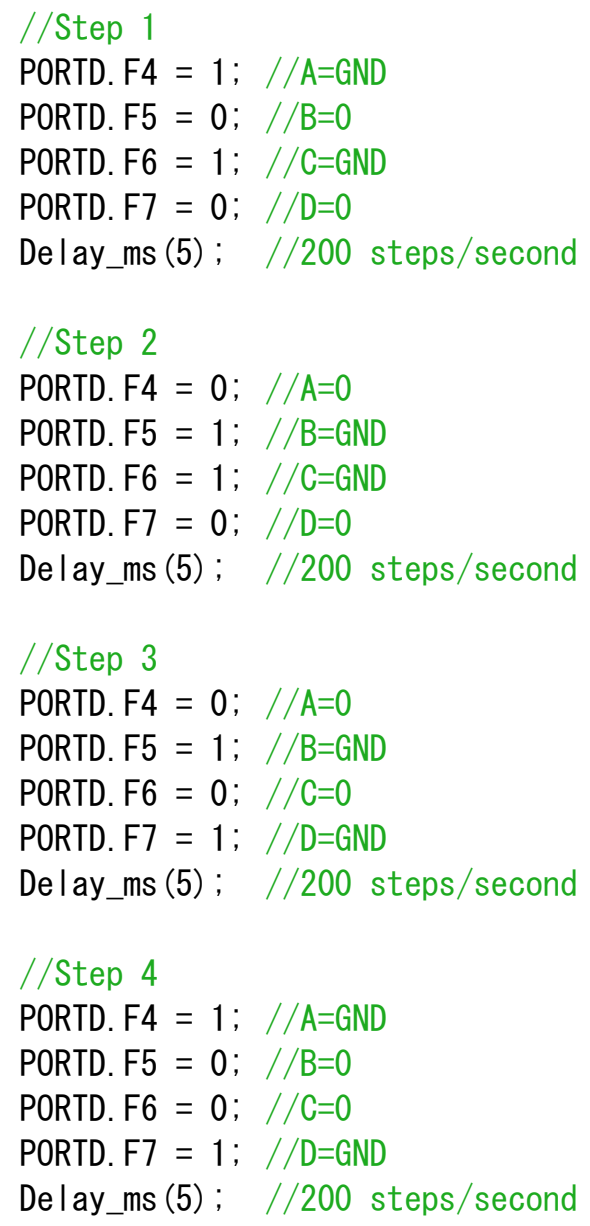




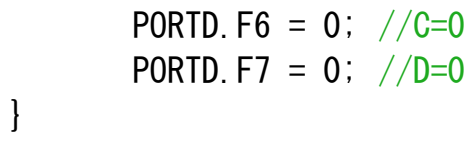


Alejandro Catalá Espí

The previous code shows how the software controls what ports of the microcontroller are made active or not to control when the motor moves and in what direction. Safety features have been implemented to avoid mixed-up situations in which both buttons are active simultaneously, and to avoid consuming power when none of the buttons is active and the motor is idle. 


\section{Annex C}

\section{Matlab Code}

This Annex presents the Matlab code programmed to: (1) automatically calibrate the samples spectra with the calibration parameters extracted from the acquisition of calibration lamps and Raman standards spectra, and (2) extract the SNR of a Raman peak from the spectra in a directory. 

The following text presents the Matlab code for the extraction of the parameters to apply in the calibration of Raman spectra taken with a particular spectrometer.

\section{function $[\mathrm{P}$, laserWL, Hnorm $]=$ cal ibparams}

\% Function for extracting the calibration parameters based on Neon, Hg-Ar and Cyclohexane spectra acquired with the descalibrated spectrometer and the theoretical list of peaks. Every spectra in pixels.

$\%$ Steps:

$\% \quad$ 1) Wavelength calibration with Neon (and $\mathrm{Hg}$ ).

\% 2) Intensity calibration with white lamp.

\% 3) Raman shift calibration with $801.3 \mathrm{~cm}^{-1}$ peak of Cyclohexane.

\% 4) Calibration verification with Raman standard (Cyclohexane).

$\%$ This function returns a mat file containing three variables:

$\% \quad P$ : polynom for the wavelength correction $[1 \mathrm{xN}]$.

$\% \quad$ laserWL: wavelength of the excitation source in $\mathrm{nm}$ [1 $1 \mathrm{x} 1]$

$\% \quad H n o r m$ : normalized ( 0 to 1 ) transfer function of the optical path [pixels (Nx1) normIntens (Nx1)]

format short;

laserWL $=532.1$

laserWL2 = 532. 0 ;

$\%$ 1) Wavelength calibration with Neon (and $\mathrm{Hg}$ )

$\% \mathrm{Hg}-\mathrm{Ar}$

$\%$ Get the Hg-Ar descalibrated spectrum (pix)

[hgPixFile, hgPixPath, FI] = uigetfile('*.xy', ' Select Hg-Ar descalibrated pixel-expressed file');

hg $=\operatorname{importSpec}($ strcat (hgPixPath, hgPixFile)); 
\% Import the list of $\mathrm{Hg}$ peaks (theoretical wavelengths)

[hglistfile, hglistpath, FI] = uigetfile ('*. mat', 'Select the list of Hg peaks');

load (strcat (hgl istpath, hgl istfile), '-mat') ;

$\%$ Select Hg peaks in the descalibrated spectrum

figure (1):

plot (hg $\left.(:, 1), \operatorname{hg}(:, 2), r^{\prime}\right)$;

$x \lim ([\mathrm{hg}(1,1) \mathrm{hg}($ length $(\mathrm{hg}), 1)])$;

[xHgPix, yHgPix] $=$ ginput (3); \%Select the 3 peaks

\% For each of the xHgPix peaks, search the pixel that corresponds to the maximum intensity in the surroundings of the selected peak

for $\mathrm{i}=1$ : length $(\mathrm{xHgPi})$

hgRange $=$ find $\left(h g(:, 1)\left\langle=x H g P i x(i), 1,{ }^{\prime}\right|\right.$ ast' $)-5:$ find $(h g(:, 1)\rangle=x H g P i x(i), 1$, first' $)+5$; \%Expressed in hg indexes.

yHgMax $=\max ($ hg (hgRange, 2$)$ );

$x$ HgMax $=h g(h g R a n g e(f i n d(h g(h g R a n g e, 2)==y H g M a x)), 1) ; \%$ Pixel number where the maximum is

$x \operatorname{HgPix}(\mathrm{i})=x \operatorname{HgMax}$;

end

clear xHgMax yHgMax hgRange

$\%$ For each maximum, interpolate and find the maximum with better precision in pixels (sub-pixel accuracy)

for $\mathrm{i}=1$ : length $(\mathrm{xHgPix})$

$\%$ Take a range from $x H g P i x(i)-10: x H g P i x(i)+10$ to interpolate

hgRange $=$ find $(h g(:, 1)==x H g P i x(i))-10:$ find $(h g(:, 1)==x H g P i x(i))+10 ; \%$ in index values

xInterp $=$ hgRange (1):0.001: hgRange (length (hgRange)); \% milipixel precision

$y$ Interp = interp1 (hg (hgRange, 1), hg (hgRange, 2), $x$ Interp, ' spl ine' ) ;

$x \operatorname{HgPix}(i)=x \operatorname{Interp}(f i n d(y \operatorname{Interp}==\max (y \operatorname{Interp}))) ; \quad \%$ Returns the position of the interpolated maximum

clear hgRange $x$ Interp yInterp

end

$\%$ NEON 
$\%$ Get the Neon descalibrated spectrum (pix)

[neonPixFile, neonPixPath, FI] = uigetfile('*.xy', ' Select Neon descalibrated pixel-expressed file');

neon $=$ importSpec (strcat (neonPixPath, neonPixFile) );

$\%$ Import the list of Neon peaks (theoretical wavelengths)

[neonlistfile, neonlistpath, FI] = uigetfile ('*. mat', 'Select the list of Neon peaks');

load (strcat (neonl istpath, neonl istfile), '-mat');

$\%$ Select Neon peaks in the descalibrated spectrum

figure (3) ;

$\operatorname{plot}\left(\right.$ neon $(:, 1)$, neon $\left.(:, 2), ' r^{\prime}\right)$;

$x \lim ([$ neon $(1,1)$ neon (length (neon), 1$)])$;

[xNeonPix, yNeonPix]=ginput (20); \%Select the 20 peaks

$\%$ For each of the xNeonPix peaks, search the pixel that corresponds to the maximum intensity in the surroundings of the selected peak

for $i=1$ : length ( $x$ NeonPix)

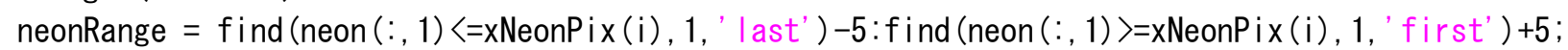

$\mathrm{yNeonMax}=\max ($ neon $($ neonRange, 2$))$;

$x$ NeonMax $=$ neon $($ neonRange $(f$ ind $($ neon $($ neonRange, 2$)==y$ NeonMax $)), 1) ; \quad \%$ Pixel number where the maximum is

$\mathrm{xNeonPix}(\mathrm{i})=\mathrm{xNeonMax}$;

end

clear xNeonMax yNeonMax neonRange

\% For each maximum, interpolate and find the maximum with better precision in pixels (sub-pixel accuracy)

for $i=1$ : length ( $x$ NeonPix)

$\%$ Take a range from $x H g P i x(i)-10: x H g P i x(i)+10$ to interpolate

neonRange $=$ find $($ neon $(:, 1)==x \operatorname{NeonPix}(i))-10$ : find $($ neon $(:, 1)==x \operatorname{NeonPix}(i))+10 ; \%$ in index values

xInterp $=$ neonRange $(1): 0.001$ : neonRange (length (neonRange) $) ; \%$ milipixel precision

yInterp = interp1 (neon (neonRange, 1), neon (neonRange, 2), xInterp, 'spline' );

$x \operatorname{NeonPix}(i)=x \operatorname{Interp}(f i n d(y \operatorname{Interp}==\max (y \operatorname{Interp}))) ; \quad \%$ Returns the position of the interpolated maximum

clear hgRange xInterp yInterp 
$\%$ Join Iists and selected peaks

selPeaks = vertcat $(x H g P i x, x$ NeonPix $)$;

theoryPeaks $=$ vertcat $($ hgWL I ist, neonWL I ist)

$\%$ Take polyfit with the real and the theoretically calculated data

$P=$ polyfit (selPeaks, theoryPeaks, 5); \% Gives wavelengths vs. pixels

\% 2) Laser wavelength extraction from stable peak of Cyclohexane

$\%$ Raman shift of the main peak of Cyclohexane

cycloPeak = 801.3;

\% Load the Cyclohexane descalibrated spectrum (pix)

[cycloPixfile, cycloPixpath, FI] = uigetfile('*. xy', 'Select Cyclohexane descalibrated pixel-expressed file'); cyclo = importSpec (strcat (cycloPixpath, cycloPixfile));

\% Import the list of $\mathrm{Hg}$ peaks (theoretical wavelengths)

[cyclolistfile, cyclolistpath, FI] = uigetfile('*. mat', 'Select the list of Cyclohexane peaks');

load (strcat (cyclolistpath, cyclolistfile), '-mat');

$\%$ Select the peaks in the figure

figure (4);

plot $\left(\operatorname{cyclo}(:, 1), \operatorname{cyclo}(:, 2), \mathrm{g}^{\prime}\right)$;

$x \lim ([\operatorname{cyclo}(1,1)$ cyclo (length (cyclo), 1) ]) ;

$[x$ CycloPix, yCycloPix]=ginput $(7+1)$; \%Select the laser and the peaks

$\%$ For each of the $\mathrm{xCycloPix}$ peaks, search the pixel that corresponds to the maximum intensity in the surroundings of the selected peak

for $i=1$ : length ( $x$ CycloPix)

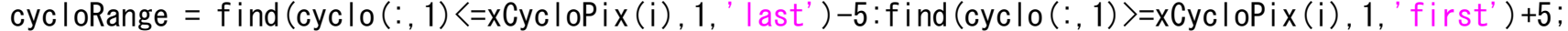


yCycloMax $=\max ($ cyclo (cycloRange, 2));

$x$ CycloMax $=\operatorname{cyclo}(\operatorname{cycloRange}($ find $(\operatorname{cyclo}($ cycloRange, 2$)==y C y c l o M a x)), 1)$;

$x$ CycloPix $(i)=x$ CycloMax

end

clear xCycloMax yCycloMax cycloRange

\% For each maximum, interpolate and find the maximum with better precision in wavelength (sub-pix accuracy)

for $i=1$ : length $(x$ CycloPix)

$\%$ Take a range from $x$ CycloPix $(i)-10: x C y c l o P i x(i)+10$ to interpolate

if find $(\operatorname{cyclo}(:, 1)==x \operatorname{Cyc} \mid o P i x(i))-10<=0$

cycloRange $=$ find $(\operatorname{cyclo}(:, 1)==x \operatorname{CycloPix}(\mathrm{i}))$

find $(\operatorname{cyclo}(:, 1)==x \operatorname{CycloPix}(i))+1$ : find $(\operatorname{cyclo}(:, 1)==x \operatorname{CycloPix}(i))+f i n d(\operatorname{cyclo}(:, 1)==x \operatorname{CycloPix}(i))+1 ; \quad \%$ in index values

else

cycloRange $=$ find $(\operatorname{cyclo}(:, 1)==x \operatorname{CycloPix}(i))-10:$ find $(\operatorname{cyclo}(:, 1)==x \operatorname{CycloPix}(i))+10 ; \quad \%$ in index values

end

xInterp $=\operatorname{cyclo}($ cycloRange $(1)): 0.001:$ cyclo $($ cycloRange $($ length (cycloRange $))) ; \%$ mil inm precision

yInterp = interp1 (cyclo (cycloRange, 1$),$ cyclo (cycloRange, 2), xInterp, 'spline');

$x \operatorname{CycloPix}(\mathrm{i})=\operatorname{xInterp}(\mathrm{find}(\mathrm{y} \operatorname{Interp}==\max (\mathrm{yInterp}))) ; \quad \%$ Returns the position of the interpolated maximum

clear cycloRange xInterp yInterp

end

while $(\operatorname{round}(1000 * \mid$ aserWL $) / 1000 \sim=\operatorname{round}(1000 * \mid$ aserWL2) $/ 1000)$

\% Calculate the laser wavelength from the lamps and convert pix to wavelength for the Cyclohexane peaks knowing their theoretical RS

laserWL = polyval $(\mathrm{P}, \mathrm{xCyc|oPix}(1))$;

cycloWL = 1. /(1/laserWL-1e-7. *cycloRS $)$;

$\%$ With cycloWL and the wavelength of the lamps peaks, new $\mathrm{P}$

clear selPeaks theoryPeaks

selPeaks = vertcat $(x$ CycloPix, xHgPix, xNeonPix);

theoryPeaks = vertcat (laserWL, cycloWL, hgWL I ist, neonWL I ist); 
$\%$ Take polyfit with the real and the theoretically calculated data clear $P$

$P=$ polyfit (selPeaks, theoryPeaks, 5); \%Gives wavelengths vs. pixels

$\%$ Recalculate laser wavelength from $\mathrm{P}$

laserWL2 = polyval $(\mathrm{P}, \mathrm{xCycloPix}(1))$;

end

\% 3) Intensity calibration with white lamp

$\%$ Get the white lamp descalibrated spectrum (pix)

[whitePixfile, whitePixpath, FI] = uigetfile ('*.xy', 'Select White Lamp descalibrated pixel-expressed file'): white $=$ importSpec $($ strcat $($ whitePixpath, whitePixfile) $)$;

$\%$ Convert pixel values to wavelength values using $\mathrm{P}$

whiteWL $=[$ polyval $(P$, white $(:, 1))$ white $(:, 2)]$;

$\%$ Import the real response of the white lamp

[realWhitefile, realWhitepath, FI] = uigetfile ('*. xy', 'Select White Lamp theory file');

realWhite $=$ importSpec $(\operatorname{strcat}($ realWhitepath, realWhitefile $))$;

$\%$ Create interpolated version of theory spectrum (in miliWL)

realWhiteIntX $=$ realWhite $(1,1): 0.001$ : realWhite $($ length $($ realWhite $), 1)$;

realWhiteIntY $=$ interp1 $($ realWhite $(:, 1)$, realWhite $(:, 2)$, realWhiteIntX, 'spline' $)$;

$\%$ Select the values of the interpolated theoretical spectrum which match the converted to WL realWhite2 = zeros (length (whiteWL), 2);

for $i=1$ : length (whiteWL)

realWhite2 $(\mathrm{i}, 1)=$ round $($ whiteWL $(\mathrm{i}, 1) * 1000) / 1000$;

end

realWhite2 $(i, 2)=$ realWhiteIntY $($ find $($ round $($ realWhiteInt $X * 1000)==$ realWhite2 $(i, 1) * 1000))$; 
$\%$ Now, divide to obtain the transfer function of the optical system $\mathrm{H}=$ zeros (length (whiteWL), 2);

$H=[$ realWhite2 $(:, 1)$ whiteWL $(:, 2) . /$ realWhite2 $(:, 2)]$;

$\%$ Normalize the transfer function to its maximum

Hnorm $=[H(:, 1) H(:, 2) . / \max (H(:, 2))]$;

end

\section{function spectrum $=$ importSpec $(\mathrm{fi}$ lename $)$}

\% Function for importing an $x y$-formatted spectrum to an array in Matlab

$\%$ Open the file for reading

file $=$ fopen $\left(f i l e n a m e,{ }^{\prime} r^{\prime}\right)$;

$\%$ Create possible separators between columns

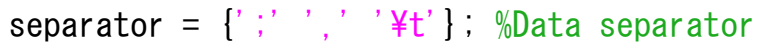

\% Import content of the file to a variable spectrum

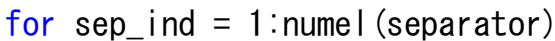

spectrum=fscanf (file, strcat ('\%f', separator $\left.\left\{\operatorname{sep}_{-} i n d\right\}, ' \% f ¥ n^{\prime}\right),[2$ inf $]$ ); spectrum=spectrum' ;

$\%$ If one of the separators is the good one, no need to use the others

if (numel (spectrum) $>1$ )

end

end

$\%$ If none of the separators is valid... error message is shown

if (numel (spectrum) $<=1$ ) 
error ('Spectrum format not supported. Separator is not, ; or ¥t.') ;

end

fclose (file) ;

end 
The next functions are used to perform the calibration of the pixel-expressed Raman spectra on the basis of the calibration parameters extracted with the code just presented above.

\section{function cal ibrate (spectrometer, cal ibtype, normtype, inf, sup)}

\% Function for calibrating (and cutting) several spectra: spectrometer:

$$
\text { 'bwtek' }
$$

calibtype: calibration options

$0=$ none

1 = wavelength

2 = wavelength + intensity

normtype: normalization options

$0=$ no normalization

$1=$ normalization by integration time

2 = normalization by max intensity

cutting limits:

inf $=$ value of the lower limit $\left(\right.$ in $\left.\mathrm{cm}^{-1}\right)$

sup = upper limit value $\left(\mathrm{cm}^{-1}\right)$

Multiple selection is possible

\% First select the xy spectrum/a to calibrate

[specfile, specpath, specfilter] = uigetfile('*. txt', 'Select RAW spectra file/s to calibrate', 'MultiSelect' ,'on');

$\%$ Select the calibration files

[calibfile, calibpath, calibfilter] = uigetfile('*. mat', 'Select the mat file with the calibration variables to be used');

$\%$ Then import and calibrate one by one. Then save

if strcmp (spectrometer, 'bwtek') 
if iscell (specfile)==1

for $i=1$ : length (specfile)

specIN = importBWTEK (strcat (specpath, specfile $\{i\})$, normtype);

specOUT = calibSpec (specIN, cal ibtype, strcat (cal ibpath, cal ibfile) );

specCut = cutSpec (spec0UT, inf: sup);

end

else if iscell (specfile) $==0$

specIN = importBWTEK (strcat (specpath, specfile), normtype);

spec0UT = calibSpec (specIN, calibtype, strcat (calibpath, calibfile));

specCut = cutSpec (spec0UT, inf: sup);

saveSpec (specCut, specpath, strcat (specfile (1: find (specfile==' ' ) -1), ' xyc' )) ;

end

end

end

end

\section{function spectrum = importBWTEK ( $\mathrm{f}$ i lename, normtype)}

\% Function for importing BWTEK txt file into xy

$\%$ Open the file for reading

file = fopen (filename, 'r');

$\%$ Skip the first header I ines and get the values

c = textscan (file, '\%s', 'Delimiter' , ' ¥n', 'BufSize', 65536):

spectrum = zeros (size (c $\{1\}, 1)-f$ ind (strcmp (c $\{1\}$, 'Pixel; Wavelength; Wavenumber; Raman Shift;Dark; Reference;Raw data \#1;Dark Subtracted

\#1;\%TR \#1; Absorbance \#1;Raw data \#2; Dark Subtracted \#2;\%TR \#2; Absorbance \#2; Raw data \#3;Dark Subtracted \#3;\%TR \#3;Absorbance \#3; Lamp

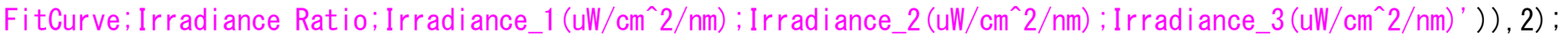

$\mathrm{j}=1$; 
for i=find (strcmp (c\{1\}, 'Pixel; Wavelength; Wavenumber; Raman Shift; Dark; Reference; Raw data \#1;Dark Subtracted \#1;\%TR \#1;Absorbance \#1; Raw data \#2; Dark Subtracted \#2;\%TR \#2; Absorbance \#2; Raw data \#3;Dark Subtracted \#3;\%TR \#3; Absorbance \#3; Lamp

FitCurve; Irradiance Ratio; Irradiance_1 (uW/cm^2/nm); Irradiance_2 (uW/cm²/nm); Irradiance_3 (uW/cm²/nm)' )) +1:1: size (c $\{1\}, 1)$ indexes = find $\left(\mathrm{c}\{1\}\{\mathrm{i}\}==^{\prime} ;{ }^{\prime}\right)$;

$\operatorname{spectrum}(j, 1)=\operatorname{str} 2 \operatorname{double}(\mathrm{c}\{1\}\{\mathrm{i}\}(1: \operatorname{indexes}(1)-1))$;

$\operatorname{spectrum}(j, 2)=\operatorname{str} 2 d o u b l e(c\{1\}\{i\}(i n d e x e s(7)+1: i n d e x e s(8)-1)) ;$

$\mathrm{j}=\mathrm{j}+1$;

clear indexes

end

$\%$ Normal ize

switch normtype

case 1

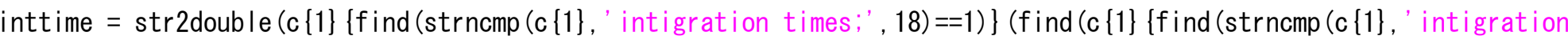

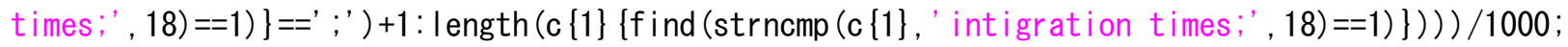

case 2 spectrum $=[\operatorname{spectrum}(:, 1)$ spectrum $(:, 2) . /$ inttime $]$;

$\max i=\max (\operatorname{spectrum}(:, 2))$;

spectrum $=[\operatorname{spectrum}(:, 1) \operatorname{spectrum}(:, 2) . / \operatorname{maxi}] ;$

end

$\%$ Close file

fclose (file);

end

\section{function specOUT = calibSpec (specPix, option, calibfile)}

\% Function for calibrating the spectrum passed as parameter and previously

$\%$ loaded in the workspace 
specRS = calibSpec (specPix, option)

specPix: pixel-expressed spectrum already in workspace

option:

$$
\begin{aligned}
& 0=\text { none } \\
& 1=\text { wavelength } \\
& 2=\text { wavelength }+ \text { intensity }
\end{aligned}
$$

$\%$ Inside this function a . mat file with the calibration parameters is asked

$\%$ from the user.

$\%$ This file MUST be comprised of the following three variables:

$\% \quad P$ : polynom for the wavelength correction $[1 \mathrm{xN}]$

$\%$ laserWL: wavelength of the excitation source in $\mathrm{nm}[1 \times 1]$

$\%$ Hnorm: normalized (0 to 1 ) transfer function of the optical path

$\% \quad[$ pixels $(\mathrm{Nx} 1)$ normIntens $(\mathrm{Nx} 1)]$

$\%$ Load the mat file just selected load (calibfile);

\% Calibrate according to option

specWL = [polyval $(P, \operatorname{specPix}(:, 1)) \operatorname{specPix}(:, 2)]$

switch option

case 1

$\operatorname{spec}=[1 \mathrm{e} 7 *(1 /$ laserWL-1. $/ \operatorname{specWL}(:, 1)) \operatorname{specWL}(:, 2)] ;$

case 2

$\operatorname{spec}=[1 \mathrm{e} 7 *(1 /$ |aserWL-1. $/ \operatorname{specWL}(:, 1)) \operatorname{specWL}(:, 2) . /$ Hnorm $(:, 2)]$;

case 0

end

spec $=$ specPix

$\%$ Interpolate so the spectrum goes in steps of $1 \mathrm{~cm}^{-1}$ (integer)

$x i=f \operatorname{loor}(\operatorname{spec}(1,1)): 1:$ floor $(\operatorname{spec}($ length $(\operatorname{spec}), 1))$;

$\operatorname{spec0UT}=\left[\mathrm{xi}^{\prime}(i \operatorname{interp1}(\operatorname{spec}(:, 1), \operatorname{spec}(:, 2), \mathrm{xi}))^{\prime}\right]$; 
0
0
0
0
$\frac{0}{\pi}$
$\frac{\pi}{\pi}$
$\sum^{\pi}$
0
0
0
0
$\frac{1}{4}$ 


\section{SNR Calculation}

function SNRdirauto (RSrange)

$\%$ This function allows the calculation of the SNR of a peak in a collection

$\%$ of spectra from the same material (noise region is calculated only once)

$\%$ SNR (RSrange)

\% range: range of data from where to extract peak and noise values

\% (example: 700:1500)

\% Select directory from where to get the spectra

sourceDir = uigetdir (' ', 'Select source directory');

cd (sourceDir)

I istSpec $=\operatorname{dir}($ sourceDir)

\% Open file to save the information on SNR

fid = fopen (' SNRvalues. txt', ' $w$ ') ;

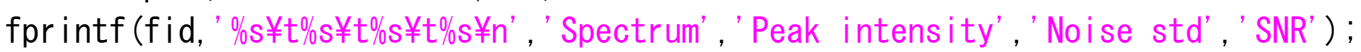

$\%$ Import spectra

$i=1$;

while (| istSpec (i). isdir==1 || |istSpec (i). name (1)==' .' )

$$
\mathrm{i}=\mathrm{i}+1
$$

end

for $\mathrm{j}=1$ : length (listSpec) $-\mathrm{i}+1$

$\%$ Import spectrum

spectrum $=$ importSpec $(I i$ stSpec $(j+i-1) \cdot$ name $) ;$

$\%$ Open and prepare figure

figure(1)

set (gcf, ' units', 'normal ized', 'outerposition', [ll $\left.\begin{array}{llll}0 & 0 & 1\end{array}\right]$ )

$\%$ Plot spectrum

minspec $=$ find $\left(\operatorname{spectrum}(:, 1)<=m i n(R S r a n g e), 1,{ }^{\prime}\right.$ last' $)$; 


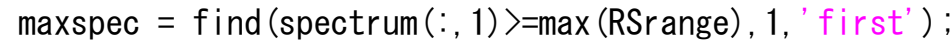

plot (spectrum (minspec:maxspec, 1 ), spectrum (minspec:maxspec, 2)) :

if $\mathrm{j}==1$

\% Select zone to extract the noise from (2 ginputs)

$[$ xnoise, ynoise $]=$ ginput $(2)$;

end

$\%$ Calculate the standard deviation from that region (subtracting baseline!)

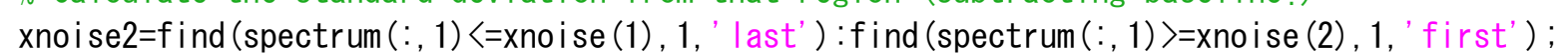

$x=\operatorname{spectrum}(x n o i s e 2,1)$;

$y=\operatorname{spectrum}(x n o i s e 2,2)$;

$p=$ polyfit $(x, y, 1) ; \quad \%$ Linear fitting

yfit $=$ polyval $(p, x) ; \quad \%$ Baseline of the selected noise region

y2 = y-yfit; \% Subtract basel ine to that region of noise

$\mathrm{s}=\operatorname{std}(\mathrm{y} 2) ; \quad \%$ Standard deviation of the noise

$\%$ Select baseline of the peak (1 ginput). From this value, calculate height

$[$ xpeak, $y p e a k]=\operatorname{ginput}(1)$;

$\%$ xpeax will serve to get the max of the peak

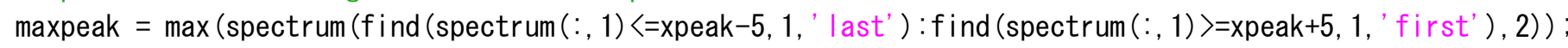

$\%$ ypeak to get the height of the baseline

intens $=$ maxpeak - ypeak

$\%$ Calculate SNR value

SNR = intens $/ \mathrm{s}$;

$\%$ Write in file

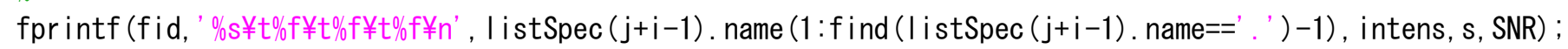

\% Clear variables and close figures

clear spectrum $x$ y xpeak ypeak maxpeak intens s SNR;

close all;

end 
fclose (fid) : 


\section{Appendix A}

\section{Publications, Abstracts,}

\section{References and Merits}

In this chapter, the published articles to which I have contributed, the abstracts I have presented (or I am present in) and the references used along this manuscript are listed. A short but personally emotive section is included to list the merits and honors received during the course of this PhD Thesis. 



\section{Publications}

Catalá-Espí, A. (2013). Vida, Marte y ExoMars. Revista de Ciencias. 3, pp. 17 - 20. Universidad de Valladolid. ISSN 2255-5943

Gázquez, F., Rull, F., Calaforra, J., Venegas, G., Manrique, J., Sanz, A., Medina, J., Catalá-Espí, A., Sansano, A., Navarro, R., Forti, P., De Waele, J., \& MartínezFrías, J. (2014). Caracterización mineralógica y geoquímica de minerales hidratados de ambientes subterráneos: implicaciones para la exploración planetaria. Estudios Geológicos, 70(2): e009 doi: 10.3989/egeol.41688.314

Gázquez, F., Rull, F., Calaforra, J., Venegas, G., Manrique, J., Medina, J., Catalá-Espí, A., Sansano, A., Navarro, \& Martínez-Frías, J. (2014). Evaluating capabilities of Mars-related techniques for the study of hydrated sulfate minerals from Mina Rica mine (SE, Spain). Planetary and Space Science, Elsevier. (submitted).

Lopez-Reyes, G., Rull, F., Venegas, G., Westall, F., Foucher, F., Bost, N., Sanz, A., Catalá-Espí, A., Vegas, A., Hermosilla, I., Sansano, A. \& Medina, J. (2013). Analysis of the scientific capabilities of the ExoMars Raman Laser Spectrometer instrument. European Journal of Mineralogy, 25(5), 721-733.

\section{Technical documentation}

Catalá-Espí, A., López-Reyes, G., Sanz, A. \& Vegas, A. (2010). RLS-CAB-TN-005: Raman focus vs. Mean focus measurements on powdered samples. ExoMars Project Consortium.

Catalá-Espí, A., López-Reyes, G. \& Rull, F. (2013). RLS-UVA-TN-004: Study on thermolability of samples under Martian conditions due to laser effects. ExoMars Project Consortium.

López-Reyes, G., Catalá-Espí, A. \& Rull, F. (2009). RLS-CAB-TN-003: Autofocus evaluation Technical Note. ExoMars Project Consortium. 
López-Reyes, G., Catalá-Espí, A., Hermosilla, I., Vegas, A. \& Rull, F. (2011). RLSCAB-TN-007: Considerations and improvements proposed for RLS Mission Operation. ExoMars Project Consortium.

López-Reyes, G., Catalá-Espí, A., Sansano, A. \& Rull, F. (2013). RLS-UVA-TN-003:

Science considerations and test proposals for the evaluation and optimization of RLS during BB STEP 2b. ExoMars Project Consortium.

López-Reyes, G., Catalá-Espí, A. \& Rull, F. (2013). RLS-UVA-RP-003: SPDS-RLS E2E Test Report. ExoMars Project Consortium.

Sanz, A., López-Reyes, G., Catalá-Espí, A. \& Rull, F. (2010). RLS-CAB-TN-006: Spectral signal to noise ratio (SNR) estimation for Raman spectroscopy. ExoMars Project Consortium.

\section{Abstracts}

Catalá, A., López Reyes, G., Rull Pérez, F., \& Vegas Hernández, A. (2011, October). Design of a small Martian chamber for simulating the RLS-ExoMars operation mode. In EPSC-DPS Joint Meeting 2011 (Vol. 1, p. 708).

Catalá-Espí, A., Lefebvre, C., Sobrón, P., Léveillé, R., Koujelev, A., Blake, D. F., \& Amundsen, H. E. F. (2013, March). 3D Chemical Mapping Using LIBS: Implications for Geochemical Investigations on Mars. In Lunar and Planetary Institute Science Conference Abstracts (Vol. 44, p. 2726).

Hermosilla, I., Lopez-Reyes, G., Catalá, A., Sanz, A., Llanos, D. R., \& Rull, F. (2012, September). Raman spectra processing algorithms and database for RLSExoMars. In European Planetary Science Congress 2012 (Vol. 1, p. 567).

Lalla, E., Mateo-Marti, E., Catalá, A., Hermosilla, I., Medina, J., Martínez-Frías, J., \& Rull, F. (2011). Analysis and absorption-interaction of Amino-acids on basaltic mineral surfaces under Martian Atmospheres conditions. Origins 2011 International Conference. Montpellier, France.

Lalla, E., Rull, F., Martinez-Frías, J., Medina, J., Sansano, A., Sanz, A., Catalá, A. \& Losada, J. A. (2012, September). Raman analysis of volcanic basalt from 
Tenerife and its hydrothermal alteration: A volcanic terrestrial analogue. In European Planetary Science Congress 2012 (Vol. 1, p. 738).

López-Reyes, G., Rull, F., Catala, A., Sanz, A., Medina, J., Hermosilla, I., Lafuente, B. (2012). A simple statistical method for the pseudo-quantification of mineral phases within the ExoMars Raman RLS instrument. Geo-Raman Xth Meeting. Nancy, France.

Rull, F., Lopez, G., Catala, A., Sanz, A., Sansano, A., Medina, J., \& Martinez Frias, J. (2010, May). Spectroscopic Raman study of powdered mineral samples in automatic mode for ExoMars mission operation simulation. In EGU General Assembly Conference Abstracts (Vol. 12, p. 14164).

Rull, F., Maurice, S., Diaz, E., Tato, C., Pacros, A., \& Team, R. (2011, March). The Raman laser spectrometer (RLS) on the ExoMars 2018 rover mission. In Lunar and Planetary Institute Science Conference Abstracts (Vol. 42, p. 2400).

Rull, F., Maurice, S., Diaz, E., Lopez, G., \& Catala, A. (2013, March). Raman Laser Spectrometer (RLS) for ExoMars 2018 Rover Mission: Current Status and Science Operation Mode on Powdered Samples. In Lunar and Planetary Institute Science Conference Abstracts (Vol. 44, p. 3110).

Rull, F., Gázquez, F., Calaforra, J., Venegas, G., Catalá-Espí, A., Sansano, A., Medina, J. \& Martínez-Frías, J. (2013). Caracterización mineralógica y geoquímica de sulfatos hidratados mediante espectroscopías Raman e IR, LIBS y DRX-FRX en la mina Quien Tal Lo Pensara (Almería) Implicaciones para la misión EXOMARS. IX Congreso Ibérico y XI Congreso Nacional de Geoquímica. Soria, Spain.

\section{Oral Communications}

Catalá-Espí, A. (2010). Exploración robótica de Marte "made in Spain". III Congreso de Estudiantes de la Facultad de Física (COEFFIS).

Catalá-Espí, A. (2010). El 2010 astronómico en 5 titulares. Museo de la Ciencia de Valladolid. 
Alejandro Catalá Espí

Catalá-Espí, A. (2013). Mars Simulation Chamber video demonstration. World Space Week 2013. Österreichisches Weltraum Forum

\section{Merits, Honours and transversal activities}

Award of the Audience: Best Oral Communication for Exploración robótica de Marte "made in Spain".

Regional Coordinator for Castilla y León of the World Space Week 2013:

Calle, A., Rodríguez, I. \& Catalá-Espí, A. (2013). La exploración de Marte: entre la decepción y la expectación. World Space Week 2013. Museo de la Ciencia de Valladolid.

Catalá-Espí, A., Rull, F. \& Unidad Asociada UVa-CSIC-CAB Team (2013). Jornada de Puertas Abiertas Unidad Asociada UVa-CSIC-CAB. World Space Week 2013.

Paredes, I. (2013). Los secretos del Clima Espacial. World Space Week 2013. 


\section{References}

Allwood, A. C., Walter, M. R., Kamber, B. S., Marshall, C. P., \& Burch, I. W. (2006). Stromatolite reef from the Early Archaean era of Australia. Nature, 441(7094), 714-718.

Allwood, A. C., Grotzinger, J. P., Knoll, A. H., Burch, I. W., Anderson, M. S., Coleman, M. L., \& Kanik, I. (2009). Controls on development and diversity of Early Archean stromatolites. Proceedings of the National Academy of Sciences, 106(24), 9548-9555.

Bai, Y., Yin, Y., Yang, J., Qing, C., \& Zhang, W. (2011). Raman study of pure, C-coated and Co-doped LiFePO4: thermal effect and phase stability upon laser heating. Journal of Raman Spectroscopy, 42(4), 831-838.

Ball, A. J. (2013). ExoMars Pasteur Payload Experiment Interface Requirements Document (PPL E-IRD), EXM-PL-IRD-ESA-00001 Issue 8, Rev. 0.

Bianciardi, G., Miller, J. D., Straat, P. A., \& Levin, G. V. (2012). Complexity analysis of the Viking labeled release experiments. International Journal of Aeronautical and Space Sciences, 13(1), 14-26.

Bibring, J. P., Langevin, Y., Mustard, J. F., Poulet, F., Arvidson, R., Gendrin, A., ... \& Neukum, G. (2006). Global mineralogical and aqueous Mars history derived from OMEGA/Mars Express data. Science, 312(5772), 400-404.

Bishop, J. L., \& Murad, E. (2005). The visible and infrared spectral properties of jarosite and alunite. American Mineralogist, 90(7), 1100-1107.

Bowie, B. T., Chase, D. B., \& Griffiths, P. R. (2000). Factors affecting the performance of bench-top Raman spectrometers. Part II: effect of sample. Applied Spectroscopy, 54(6), 200A-200A.

Burchell, M. J. (2004). Panspermia today. International Journal of Astrobiology, $3(02), 73-80$. 
Carr, M. H., \& Head III, J. W. (2010). Geologic history of Mars. Earth and Planetary Science Letters, 294(3), 185-203.

Chou, I., \& Seal, R. R. (2007). Magnesium and calcium sulfate stabilities and the water budget of Mars. Journal of Geophysical Research: Planets (19912012), 112(E11).

Christensen, P. R., Bandfield, J. L., Clark, R. N., Edgett, K. S., Hamilton, V. E., Hoefen, T., ... \& Smith, M. D. (2000). Detection of crystalline hematite mineralization on Mars by the Thermal Emission Spectrometer: Evidence for near-surface water. Journal of Geophysical Research: Planets (1991-2012), 105(E4), 9623-9642.

Cloutis, E. A., Craig, M. A., Mustard, J. F., Kruzelecky, R. V., Jamroz, W. R., Scott, A., ... \& King, P. L. (2007). Stability of hydrated minerals on Mars. Geophysical Research Letters, 34(20).

Deamer, D. W. (1985). Boundary structures are formed by organic components of the Murchison carbonaceous chondrite.

Ehlmann, B. L., Mustard, J. F., Murchie, S. L., Bibring, J. P., Meunier, A., Fraeman, A. A., \& Langevin, Y. (2011). Subsurface water and clay mineral formation during the early history of Mars. Nature, 479(7371), 53-60.

Ehrenfreund, P., \& Charnley, S. B. (2000). Organic molecules in the interstellar medium, comets, and meteorites: a voyage from dark clouds to the early Earth. Annual Review of Astronomy and Astrophysics, 38(1), 427-483.

Foucher, F., Lopez-Reyes, G., Bost, N., Rull-Perez, F., Rüßmann, P., \& Westall, F. (2013). Effect of grain size distribution on Raman analyses and the consequences for in situ planetary missions. Journal of Raman Spectroscopy, 44(6), 916-925.

Gilbert, W. (1986). Origin of life: The RNA world. Nature, 319(6055). 
Grotzinger, J. P., Sumner, D. Y., Kah, L. C., Stack, K., Gupta, S., Edgar, L., ... \& Kocurek, G. (2014). A habitable fluvio-lacustrine environment at Yellowknife Bay, Gale crater, Mars. Science, 343(6169), 1242777.

Haberle, R. M., McKay, C. P., Schaeffer, J., Cabrol, N. A., Grin, E. A., Zent, A. P., \& Quinn, R. (2001). On the possibility of liquid water on present - day Mars. Journal of Geophysical Research: Planets (1991-2012), 106(E10), 2331723326.

Halevy, I., Fischer, W. W., \& Eiler, J. M. (2011). Carbonates in the Martian meteorite Allan Hills 84001 formed at $18 \pm 4^{\circ} \mathrm{C}$ in a near-surface aqueous environment. Proceedings of the National Academy of Sciences, 108(41), 16895-16899.

Joyce, G. F., Young, R., Chang, S., Clark, B., Deamer, D., DeVincenzi, D., ... \& Walker, J. (1994). Origins of life: The central concepts.

Kasting, J. F., Whitmire, D. P., \& Reynolds, R. T. (1993). Habitable zones around main sequence stars. Icarus, 101(1), 108-128.

Klein, H. P. (1999). Did Viking discover life on Mars?. Origins of Life and Evolution of the Biosphere, 29(6), 625-631.

Klingelhöfer, G. R. D. S., Morris, R. V., Bernhardt, B., Schröder, C., Rodionov, D. S., De Souza, P. A., ... \& Arvidson, R. E. (2004). Jarosite and hematite at Meridiani Planum from Opportunity's Mössbauer spectrometer. Science, 306(5702), $1740-1745$.

Levin, G. V., \& Straat, P. A. (1976). Viking labeled release biology experiment: interim results. Science, 194(4271), 1322-1329.

Liu, Y., Wang, A., \& Freemen, J. J. (2009, March). Raman, MIR, and NIR spectroscopic study of calcium sulfates: gypsum, bassanite, and anhydrite. In Lunar and Planetary Science Conference (Vol. 40, p. 2128). 
López Reyes, G. (2012). Procesamiento espectral para la pseudocuantificación de especies minerales en espectros del instrumento Raman Laser Spectrometer de la misión ExoMars de la ESA.

Lopez-Reyes, G., Rull, F., Venegas, G., Westall, F., Foucher, F., Bost, N., ... \& Medina, J. (2013). Analysis of the scientific capabilities of the ExoMars Raman Laser Spectrometer instrument. European Journal of Mineralogy, 25(5), 721-733.

Luisi, P. L. (1998). About various definitions of life. Origins of Life and Evolution of the Biosphere, 28(4-6), 613-622.

Martel, J., Young, D., Peng, H. H., Wu, C. Y., \& Young, J. D. (2012). Biomimetic properties of minerals and the search for life in the Martian meteorite ALH84001. Annual Review of Earth and Planetary Sciences, 40, 167-193.

Matijevic, J. R., Crisp, J., Bickler, D. B., Banes, R. S., Cooper, B. K., Eisen, H. J., ... \& Wilt, D. (1997). Characterization of the Martian Surface Deposits by the Mars Pathfinder Rover, Sojourner. Science, 278(5344), 1765-1768.

McGill, G. E., \& Squyres, S. W. (1991). Origin of the Martian crustal dichotomy: Evaluating hypotheses. Icarus, 93(2), 386-393.

McKay, D. S., Gibson Jr, E. K., Thomas-Keprta, K. L., Vali, H., Romanek, C. S., Clemett, S. J., ... \& Zare, R. N. (1996). Search for Past Life on Mars: Possible Relic Biogenic Activity in Martian Meteorite ALH84001. Science, 273, 924-930.

Morris, R. V., Ruff, S. W., Gellert, R., Ming, D. W., Arvidson, R. E., Clark, B. C., ... \& Squyres, S. W. (2010). Identification of carbonate-rich outcrops on Mars by the Spirit rover. Science, 329(5990), 421-424.

Nimmo, F., \& Tanaka, K. (2005). Early crustal evolution of Mars 1. Annu. Rev. Earth Planet. Sci., 33, 133-161.

Owen, T., Biemann, K., Rushneck, D. R., Biller, J. E., Howarth, D. W., \& Lafleur, A. L. (1977). The composition of the atmosphere at the surface of Mars. Journal of Geophysical research, 82(28), 4635-4639. 
Pérez, C. "RLS-INT-SP-008: RLS Technical Specification Document" (2013) RLSExoMars internal document.

Ponnamperuma, C., Shimoyama, A., Yamada, M., Hobo, T., \& Pal, R. (1977). Possible surface reactions on Mars: Implications for Viking biology results. Science, 197(4302), 455-457.

Prasad, P. S. R., Pradhan, A., \& Gowd, T. N. (2001). In situ micro-Raman investigation of dehydration mechanism in natural gypsum. CURRENT SCIENCE-BANGALORE-, 80(9), 1203-1207.

Prieto-Taboada, N., Gómez-Laserna, O., Martínez-Arkarazo, I., Olazabal, M. Á., \& Madariaga, J. M. (2014). Raman Spectra of the Different Phases in the CaSO4-H20 System. Analytical chemistry, 86(20), 10131-10137.

Rull, F., \& Martínez-Frías, J. (2006). Raman spectroscopy goes to Mars. Spectroscopy Europe, 18(1), 18-21.

Rull, F., Lopez, G., Catala, A., Sanz, A., Sansano, A., Medina, J., \& Martinez Frias, J. (2010, May). Spectroscopic Raman study of powdered mineral samples in automatic mode for ExoMars mission operation simulation. In EGU General Assembly Conference Abstracts (Vol. 12, p. 14164).

Shebanova, O. N., \& Lazor, P. (2003). Raman study of magnetite (Fe304): laserinduced thermal effects and oxidation. Journal of Raman Spectroscopy, 34(11), 845-852.

Squyres, S. W., Grotzinger, J. P., Arvidson, R. E., Bell, J. F., Calvin, W., Christensen, P. R., ... \& Soderblom, L. A. (2004). In situ evidence for an ancient aqueous environment at Meridiani Planum, Mars. Science, 306(5702), 1709-1714.

Squyres, S. W., Arvidson, R. E., Bell, J. F., Calef, F., Clark, B. C., Cohen, B. A., ... \& Zacny, K. (2012). Ancient impact and aqueous processes at Endeavour Crater, Mars. Science, 336(6081), 570-576.

Tim, T. (2012). CS-VSM Campaign: Crushed Sample Particle Size Distribution Measurements, EXM-RM-TRP-KT-0035 Issue 1. 
Vago, J., Gardini, B., Kminek, G., Baglioni, P., Gianfiglio, G., Santovincenzo, A., ... \& van Winnendael, M. (2006). ExoMars-searching for life on the Red Planet. ESA bulletin, 126, 16-23.

Venegas, G. (2014). Raman study of sulphates formed by hydrothermal, evaporitic and weathering processes in the south east of Spain: implications for the exploration of Mars. PhD Thesis, Universidad de Valladolid.

Wächtershäuser, G. (2006). From volcanic origins of chemoautotrophic life to Bacteria, Archaea and Eukarya. Philosophical Transactions of the Royal Society B: Biological Sciences, 361(1474), 1787-1808.

Yen, A. S., Kim, S. S., Hecht, M. H., Frant, M. S., \& Murray, B. (2000). Evidence that the reactivity of the martian soil is due to superoxide ions. Science, 289(5486), 1909-1912. 


\section{Appendix B}

\section{Resumen}

Este apéndice presenta el resumen en español de esta tesis, por estar la misma escrita íntegramente en inglés para su difusión internacional. 



\section{Capítulo 1 - Introducción}

\section{Exploración planetaria}

Desde que Giovanni Schiaparelli observara los canales marcianos y Percival Lowell los interpretara erróneamente, el interés en torno a Marte y la especulación de la existencia de vida extraterrestre (incluso inteligente) en su superficie caló hondo en la cultura popular. Así se refleja en las retransmisiones radiofónicas y posteriores novelas de ciencia ficción que abordaron este tema.

Este impulso cultural trascendió al ámbito científico y con la tecnología ya madura, la NASA inició programas de exploración del planeta rojo. Comenzando con las sondas Mariner y siguiendo con los aterrizadores Viking, Marte se descubrió como un planeta hostil donde difícilmente se encontraría vida en la actualidad.

Más misiones de exploración remota e in-situ les siguieron y descubrieron que por la superficie de nuestro planeta vecino corrieron ríos que ayudaron a moldear su superficie, dejando rastros minerales como prueba de esta afirmación.

Si hubo agua en el pasado del planeta, la vida pudo haber encontrado su nicho. El objetivo de las misiones posteriores como MSL o ExoMars es descubrir lugares representativos de un pasado potencialmente habitable y tratar de encontrar trazas de esta vida pasada (o quién sabe si presente).

\section{Astrobiología y vida}

La vida, su origen, su evolución y su distribución por el Universo sigue siendo la pregunta del millón de dólares. Una cuestión que ha martirizado a la razón humana desde tiempos antiguos y a la que la Astrobiología pretende dar respuesta científica.

Ésta es una ciencia transdisciplinaria que involucra diversas ramas de la ciencia y la ingeniería para tratar de acercarnos a la respuesta definitiva.

Numerosas son las definiciones que se han dado al término vida pero sin duda la más elegante es: 
"Un sistema químico autosostenible capaz de experimentar evolución darwiniana" (Joyce, 1994)

Uno de los ingredientes imprescindibles para que la vida, tal como la conocemos, se desarrolle es agua en estado líquido. Para que esta característica se cumpla en la superficie de un cuerpo planetario, éste debe encontrarse orbitando alrededor de lo que conocemos como zona de habitabilidad de su estrella. Aquella distancia orbital que confiere al planeta las condiciones de temperatura idóneas para la existencia de agua líquida. Esta característica, sin embargo, no debe tomarse de manera aislada sino en conjunción con las condiciones climáticas del planeta en cuestión.

Satisfecho este requerimiento, el agua líquida debe ser rica en moléculas orgánicas reducidas y átomos de $\mathrm{C}, \mathrm{H}, \mathrm{O}, \mathrm{N}, \mathrm{S}$ y $\mathrm{P}$, los bloques básicos de la vida. Estos ingredientes han sido descubiertos en el espacio, tanto intragaláctico como interplanetario, dando alas a la idea de la panspermia como proceso de diseminación de la vida a través del Universo.

Sea como fuere el origen de la vida en la Tierra, endógeno o exógeno, ésta lleva aquí desde hace aproximadamente 3450 millones de años. La Astrobiología pretende entender su origen sobre la Tierra para poder comprender dónde y cómo buscarla fuera de ella.

De haberse desarrollado la vida sobre Marte, existe una ventaja en la búsqueda de las huellas más antiguas de ésta en Marte sobre la Tierra: la ausencia de tectónica de placas. Un fenómeno de reciclaje natural de la corteza continental que se produce en la Tierra pero de la que Marte carece. Las rocas sobre la superficie del Planeta Rojo tienen, por tanto, la edad de formación del planeta, permaneciendo éstas intactas salvo por los procesos de meteorización y erosión superficial, así como de impactos meteoríticos.

La evolución del clima en Marte no ha sido muy benévolo con la superficie del planeta que, en la actualidad, se encuentra continuamente bañada por radiación ionizante y agentes oxidantes que arrasarían con cualquier intento de la 
vida por resurgir en aquellos lugares donde, sucintamente, el agua líquida (en forma de salmueras) pudiera darse.

Esta hostilidad de la superficie lleva a pensar en que cualquier forma de vida, en aras de su propia supervivencia, podría haber migrado hacia la subsuperficie del planeta buscando lugares protegidos donde sobrevivir y desarrollarse.

Bajo este supuesto nace ExoMars, la primera misión de aterrizaje marciano que explorará la subsuperficie de Marte en busca de signos de vida pasada o presente que nos ayuden a explicar el origen de la vida y con éste, el nuestro propio.

\section{El Programa ExoMars y el Rover de 2018}

El Programa ExoMars consta de dos misiones que despegarán con destino Marte en 2016 y 2018, respectivamente, portando ambas cargas científicas relacionadas con la búsqueda de vida.

En 2016, el Trace Gas Orbiter (TG0) analizará la atmósfera de Marte en busca de trazas de gases que pudieran tener relación con actividades de origen biológico en la superficie del planeta. Este orbitador portará un módulo de demostración de las capacidades europeas para posar de forma controlada sobre la superficie de Marte una carga científica. Además de las capacidades científicas del TGO, éste servirá como enlace de comunicaciones bidireccional entre la Tierra y el rover de 2018.

El Rover ExoMars, que será lanzado en 2018, aterrizará en Marte con la misión de, entre otras, analizar muestras del subsuelo marciano en busca de rastros de vida pasada o presente. Las muestras serán extraídas de una profundidad de $2 \mathrm{~m}$ por un taladro modular con el que se ha dotado al rover. El pedazo de Marte que se extraerá (un cilindro de $1 \mathrm{~cm} \times 3 \mathrm{~cm}$ ) será transferido al rover para su conversión en polvo mediante un proceso de morturado. Este polvo, con una distribución de granos minerales entre $50 \mu \mathrm{m}$ y $500 \mu \mathrm{m}$, será depositado sobre un contenedor de muestras que se presentará a los diferentes instrumentos analíticos de que dispone el rover. 
El rover ExoMars es el próximo paso lógico en la exploración de Marte tras Spirit, Opportunity y Curiosity. Y es también el precursor de misiones de retorno de muestras a la Tierra, donde se dispone de capacidades analíticas más avanzadas de las que, por el momento, se pueden transportar en un vehículo autónomo robotizado.

\section{RLS-ExoMars y la espectroscopia Raman}

El Espectrómetro Raman Láser (RLS, por sus siglas en inglés) es un instrumento seleccionado como carga científica del rover ExoMars. Será uno de los encargados de analizar las muestras en polvo que se le presenten.

Los objetivos científicos de este instrumento se heredan directamente de los objetivos del Programa ExoMars: (1) trazar la historia geoquímica del planeta, y (2) potencialmente identificar moléculas orgánicas asociadas a biomarcadores procedentes de formas de vida marcianas extintas o vivientes.

Este instrumento consta de varios elementos:

- Cabezal óptico (iOH): cabezal Raman con dos caminos ópticos para emisión de luz láser de excitación y colección de luz Raman retrodispersada, con capacidad de autofoco.

- Unidad de Espectrómetro (SPU): el espectrómetro Raman en sí mismo, compuesto por el conector de entrada de la fibra de colección del cabezal óptico, la óptica de colimación, la red de difracción, la óptica de focalización y el detector CCD.

- Unidad de Control de Instrumento y Excitación (ICEU): el cerebro del instrumento, encargado de controlar la electrónica asociada al sistema de autofoco, al detector CCD, al procesado de señales, etc., y que también alberga el láser de 532nm para la excitación.

La espectroscopia Raman es una técnica analítica potente en tanto en cuanto es capaz de identificar materiales a partir de las vibraciones y rotaciones de sus moléculas constituyentes. 
Esto se consigue haciendo incidir sobre la muestra a analizar una luz monocromática (láser). Los fotones emitidos interaccionan con la nube electrónica alrededor de los enlaces moleculares de la muestra, excitándolos a estados energéticos virtuales. Tras $10^{-14} \mathrm{~s}$, cuando la molécula se desexcita, emite un fotón cuya energía corresponde a la diferencia entre la energía inicial (antes de la excitación) y la final (tras la desexcitación). Si ésta es diferente de cero, se ha producido emisión Raman. Éste es un fenómeno de dispersión inelástica, a diferencia de la dispersión de Rayleigh, y en comparación con ésta es un fenómeno muy débil: por cada $10^{8}$ fotones Rayleigh se genera uno Raman. Un espectro Raman es, por tanto, una compilación de emisiones Raman a diferentes frecuencias diferentes a la de excitación. Estos picos de emisión proporcionan información sobre las especies químicas presentes en la muestra, mediante su posición espectral (medida en $\mathrm{cm}^{-1}$ ), el nivel de cristalización o concentración en la misma (intensidad de los picos), temperatura (ratio de intensidades Stokes-AntiStokes), así como otras caracterísiticas.

Dos son las grandes ventajas de la espectroscopia Raman frente a otras técnicas: (1) no requiere de una preparación previa de la muestra y, (2) el tiempo de análisis de las muestras es del orden de segundos (o inferior).

\section{Capítulo 2 - Justificación y Objetivos}

La tarea más importante del equipo científico de un instrumento que volará en una misión de exploración espacial es la definición de los requisitos científicos del mismo, con los que se alimenta al equipo de ingeniería para el diseño y construcción del instrumento de vuelo.

Durante la construcción de los diferentes modelos de ingeniería, el equipo científico tiene que comprobar que se cumplen los requisitos impuestos y proponer modificaciones en caso contrario o cuando se detecten riesgos importantes que requieran una redefinición de los mismos. También, a nivel operativo del instrumento final, el equipo científico debe definir el protocolo de operación en cuanto al número de puntos a analizar, potencia de láser, parámetros de adquisición, etc. 
Otra pieza fundamental, ya directamente aplicable a este instrumento, está relacionada con la futura explotación de los datos científicos. Debe construirse una base de datos de espectros Raman que sirva para comparar con los tomados de muestras marcianas.

Esta base de datos podría tener que ser completada con espectros tomados en las condiciones ambientales a las que las muestras estarán sometidas durante la misión. Éste es el objetivo de los experimentos que se presentan en el Capítulo 5.

Por otra parte, y como se ha visto en el Capítulo 1, el instrumento RLS emplea un láser como fuente de interrogación de las muestras. En espectroscopia Raman, una irradiancia mayor sobre la muestra significa, por lo general, una adquisición espectral más rápida y con mayor relación señal-ruido. Pero enfocar un láser sobre una muestraInduce calentamiento en el punto de iluminación. Este calentamiento láser puede perturbar o incluso dañar la muestra, un efecto térmico indeseable que puede dificultar la correcta interpretación de los datos. El grado en que una muestra en polvo puede ser afectada por el calentamiento láser depende de muchos factores: la distribución de tamaños de grano, la conductividad térmica de la muestra, o el grado de desorden de su estructura.

La creación de una base de datos bajo las condiciones ambientales de RLSExoMars y la ejecución de experimentos científicos como los descritos, entre muchos otros, justifican el diseño y construcción de una Cámara de Simulacion de $R L S$-ExoMars, un instrumento construido pensando en la operación definida para el instrumento RLS.

El Capítulo 3 describe este instrumento y todos los sistemas y subsistemas que lo componen, así como los resultados de los tests realizados.

El Capítulo 4 aborda el diseño y construcción de un elemento que encaja a la perfección con la Cámara de Simulación de RLS-ExoMars. Y éste es el Simulador de Ciencia de RLS-ExoMars, el complemento optomecánico que pone en comunicación las muestras en la cámara con los láseres y espectrómetros fuera de ella para realizar experimentos cientiíficos. 
El Capítulo 5 trata sobre esta última cuestión: los experimentos. Presenta dos grupos de experimentos: uno relacionado con las diferencias espectrales que las condiciones ambientales relevantes de la misión puedan imponer a los espectros Raman, creando la necesidad de construir una base de datos específica para la misión; y un segundo grupo de experimentos centrado en el estudio de los efectos de la irradiancia láser sobre dos muestras termolábiles con dos comportamientos diferentes. Este grupo de experimentos fue llevado a cabo bajo condiciones de laboratorio terrestre y también bajo condiciones relevantes de RLS-ExoMars. Este cambio de ambiente enfatiza la contribución de una baja presión atmosférica en términos de disipación de calor.

El Capítulo 6 resume el trabajo realizado en esta tesis doctoral, y reflejado en este manuscrito.

Los diversos anexos reúnen información extra relacionada con el desarrollo de esta tesis tales como dibujos, código fuente y otros algoritmos.

\section{Capítulo 3 - Cámara de Simulación de RLS-ExoMars}

\section{Introducción}

Antes del trabajo presentado en esta tesis doctoral, el Simulador RLS ExoMars sólo podía operar bajo las condiciones ambientales del laboratorio en el que se ubica.

El siguiente paso en la simulación de las operaciones científicas que el instrumento RLS realizará en Marte consiste en la incorporación de las condiciones ambientales bajo las que la muestra será analizada. Esto permitirá al equipo científico realizar experimentos bajo condiciones más realistas y determinar así la influencia de éstas sobre la evolución de las propias muestras y su proceso analítico, que podría repercutir en la creación de nuevos requisitos científicos o en la revisión de los existentes.

Para alcanzar este objetivo, la Cámara de Simulación de RLS-ExoMars ha sido construida siguiendo las especificaciones de ExoMars. 


\section{Sistemas de presión y temperatura}

La presión atmosférica en Marte varía desde los 0.3mbar en la cima del Monte Olimpo hasta los 11mbar en el punto más bajo de la Hellas Planitia. Esta presión varía no sólo con la altitud del lugar sino también a lo largo del día y durante las estaciones. Aunque el lugar de aterrizaje de ExoMars está aún por determinar, en el lugar donde MSL aterrizó la presión medida (por REMS) oscila en torno a 7 mbar. La atmósfera de Marte está compuesta en más de un 95\% de $\mathrm{CO}_{2}$.

La Cámara de Simulación de RLS-ExoMars, es capaz de recrear en su interior condiciones de presión desde $10^{-3} \mathrm{mbar}$ hasta $10^{3} \mathrm{mbar}$ y una composición de $100 \%$ de $\mathrm{CO}_{2}$ se ha utilizado como aproximación a la atmósfera real.

Para la obtención de la presión de $\mathrm{CO}_{2}$ deseada en el interior de la Cámara de Simulación de RLS-ExoMars se dispone de elementos como: una bomba turbomolecular, un sensor de presión, una línea de gas con válvula electromecánica por la que se introduce el $\mathrm{CO}_{2}$ en la cámara, un controlador de presión conectado a ambos (sensor y válvula) y una válvula de restablecimiento de las condiciones de laboratorio.

En cuanto a la temperatura de la muestra durante la operación del instrumento RLS, y atendiendo al requisito PPL-ENG-ALD-0180 del documento de requisitos de ExoMars (Ball, 2013), debe mantenerse por debajo de los $-5^{\circ} \mathrm{C}$.

La Cámara de Simulación de RLS-ExoMars aloja en su interior un contenedor de muestra refrigerado que mantiene la muestra en una temperatura determinada durante el análisis de la misma.

Para conseguir la temperatura deseada en el interior de la Cámara de Simulación de RLS-ExoMars se dispone de básicamente dos elementos: una unidad de refrigeración líquida que enfría metanol hasta $-40^{\circ} \mathrm{C}$ (teóricamente), y un sensor de temperatura PT100 insertado en el contenedor de muestra y que actúa como mecanismo de realimentación para la unidad de refrigeración.

Tras ordenar el establecimiento de las condiciones de temperatura y presión en el interior de la Cámara de Simulación de RLS-ExoMars mediante el 
software específicamente diseñado para este equipo, el sistema tarda menos de 30 minutos en alcanzarlas, manteniéndose estable en esas condiciones mientras se requiera.

\section{Sistema de muestra}

Como se ha comentado anteriormente, gracias al taladro que incorpora el rover de ExoMars, se extraerán muestras sólidas de la subsuperficie marciana que se proporcionarán al sistema de preparación y distribución de muestra para su morturado y deposición sobre el contenedor de muestras. Tras esta deposición y previo a su análisis por los instrumentos científicos a los que se le presente, la muestra en polvo es aplanada para proporcionar una superficie sin demasiadas irregularidades: no más de $200 \mu \mathrm{m}$ de variación en altura para el $80 \%$ de la superficie del contenedor con no más del $50 \%$ de los granos a $100 \mu \mathrm{m}$ de esta superficie. El objetivo, ajustarse al rango de operación del mecanismo de autofoco del cabezal Raman.

El contenedor de muestra diseñado para la Cámara de Simulación de RLSExoMars se basa en el diseñado por Kayser-Threde (KT) para el rover de ExoMars, con una capacidad de $\sim 3 \mathrm{~cm}^{3}$ de muestra. Está fabricado en cobre para maximizar la transferencia térmica y aloja en su interior un volumen vacío de $\sim 4.6 \mathrm{~cm} 3$ por el que hacer fluir el líquido de refrigeración para controlar la temperatura de la muestra. Aloja además el sensor de temperatura PT100 para realimentar el sistema de refrigeración con un dato de temperatura cercano a la muestra. Su comunicación con el exterior se realiza a través de un conector LEMO para vacío.

Aparte del contenedor de muestra, se ha reproducido el sistema de aplanado de la misma, incorporando al sistema de muestra una cuchilla y un desplazador lineal motorizado que la hace avanzar y retroceder sobre la superficie del contenedor de muestra para reproducir este proceso. El desplazador lineal se encuentra adosado a uno de los puertos de vacío de la Cámara de Simulación de $R L S$-ExoMars a través del cual interactúa con la cuchilla. El control de este desplazamiento se efectúa mediante una placa electrónica externa diseñada y construida específicamente para este motor y esta aplicación. 
Para poder acceder visual y espectroscópicamente a la muestra alojada en el contenedor de muestras, se ha mecanizado la tapa de la Cámara de Simulación de $R L S$-ExoMars de forma que permita alojar una ventana óptica. Por exigencias de grosor y transparencia en la región espectral requerida, esta ventana óptica es un portaobjetos de microscopio. Se encuentra montada en una estructura de sandwich entre dos capas de goma que actúan como barrera para contener la baja presión en el interior de la cámara. Sobre este emparedado un marco metálico atornillado a la tapa de la cámara sella el conjunto.

En el sistema de deposición de muestra real se realizan varias deposiciones de muestra intercaladas con procesos de aplanado, consiguiendo de esta manera cubrir el objetivo de "planitud" de la superficie de la muestra. En el caso del sistema de muestra de la Cámara de Simulación de RLS-ExoMars, existe una única deposición previa al cierre de la cámara y un único proceso de aplanado tras alcanzar las condiciones atmosféricas deseadas en su interior.

Para el testeo de este sistema de muestra se seleccionaron dos muestras con diferente grado de hidratación: Cuarzo y Yeso. Tras el proceso de aplanado de ambas muestras se tomaron 20 puntos en cada una de tres líneas paralelas para construir un gráfico de variación de profundidad del punto de enfoque sobre el material. Los resultados obtenidos refuerzan la operación de múltiples procesos de dosificación y aplanado para suavizar la superficie.

\section{Capítulo 4 - Simulador de Ciencia de RLS-ExoMars}

\section{Introducción}

El Simulador de Ciencia de RLS-ExoMars es un sistema optomecánico compuesto por un sistema de posicionamiento XYZ y un montaje óptico que incluye un cabezal Raman. Éste hace las funciones del iOH del instrumento RLSExoMars. Con él es posible posicionarse sobre la muestra, enfocar, excitarla con luz láser concentrada sobre el grano a analizar y a través de la ventana óptica de la Cámara de Simulación de RLS-ExoMars, y recoger la luz Raman para dirigirla al espectrómetro que la disperse y detecte. 
A diferencia del sistema real en el que el contenedor de muestra se posiciona bajo los instrumentos y éstos se mantienen estáticos (salvo por los movimientos de enfoque), a éste se le ha dotado de movimiento XY para acceder a la superficie completa del contenedor de muestra, que permanece estático en el interior de la cámara. El movimiento en Z sigue utilizándose para enfocar sobre la muestra.

\section{Antecedentes: ExoMars RLS Simulator}

El Simulador de Ciencia de RLS-ExoMars está basado en su versión anterior, construido también en la Unidad Asociada UVa-CSIC para definir y testar el modo de operación del instrumento RLS: el ExoMars RLS Simulator. En esta versión original, la muestra se desplaza en XY y se la puede aplanar haciendo uso de una cuchilla colocada en un soporte externo.

La versatilidad y utilidad de esta versión original del simulador ha sido indispensable para la definición de la operación del instrumento real como por ejemplo en la definición del número de puntos mínimo a analizar para garantizar la aparición de un compuesto minoritario de una muestra heterogénea sintética en al menos un espectro Raman, el modo de enfoque, el rango de tamaños de grano óptimos... entre otros muchos experimentos.

Con este Simulador de Ciencia de RLS-ExoMars se puede dar un paso más añadiendo las condiciones ambientales reales de la misión.

\section{Simulador de Ciencia de RLS-ExoMars}

El Simulador de Ciencia de RLS-ExoMars es el compañero ideal para la Cámara de Simulación de RLS-ExoMars presentada en el capítulo 3. Aunque los elementos constituyentes de esta nueva versión del simulador no han variado con respecto a la original, se consigue reducir el volumen y masa de los mismos aumentando al mismo tiempo la resolución espacial de los movimientos, a costa de reducir el rango de movimiento. Estos elementos son:

- $\quad$ Sistema de posicionamiento XYZ: está compuesto por un controlador de motores y tres motores DC adosados a tres etapas de desplazamiento lineal 
con rango de movimiento de $25 \mathrm{~mm}$ cada una. Este rango es suficiente para cubrir la superficie del contenedor y realizar las funciones de autoenfoque.

- Sistema óptico: el sistema óptico de esta versión del simulador (basado en el de la anterior versión) se compone de: un adaptador para el cabezal Raman que se utiliza para la excitación y colección, una película divisora de haz 8:92 montada sobre un cubo para realizar funciones de espectroscopía e imagen al mismo tiempo, un sistema óptico y detector de imagen para la visualización de la muestra, un anillo adaptador de objetivo de microscopio, y un objetivo de microscopio de larga focal para focalizar la luz láser en $50 \mu \mathrm{m}$ de spot sobre la muestra y a través de la ventana óptica de la cámara.

\section{Tests}

A este montaje óptico se le realizaron una serie de tests de caracterización y calibración, más que de funcionamiento, que consistieron en: (1) la determinación de la potencia láser de salida obtenida a la salida del sistema óptico (incluyendo la ventana) en función de la potencia de entrada comandada al driver del láser mediante un medidor de potencia y con promedio de 100 medidas en cada paso de potencia; (2) la determinación del tamaño de spot del láser proyectado por el sistema óptico en el plano de la muestra y a diferentes alturas desde él para el posterior cálculo de valores de irradiancia, utilizando para ello un medidor de perfiles láser y tomando 20 medidas por valor de altura; (3) el cálculo de la irradiancia sobre la muestra mediante los valores hallados en (1) y (2); (4) la profundidad de foco Raman (medida en micras), calculada como el intervalo de alturas desde el foco en el que la relación señal-ruido del pico Raman de máxima amplitud de una lámina de Silicio es mayor y igual al 50\% de su valor en foco; (5) la determinación de la resolución espectral del espectrómetro Raman empleado para las medidas, ajustando a curvas teóricas los picos de emisión de lámparas de calibración de Hg-Ar y Ne; (6) la determinación de la función de transferencia del sistema óptico así como la realización de calibración en intensidad y longitud de onda de los espectros tomados haciendo uso de las lámparas de calibración anteriores y de líquidos estándares como el ciclohexano, así como de algoritmos de Matlab programados para automatizar y facilitar este proceso. 


\section{Capítulo 5 - Experimentos}

\section{Introducción}

Tradicionalmente, las bases de datos de espectros utilizadas para interpretar los análisis de los instrumentos a bordo de exploradores planetarios robóticos son construidas a partir de espectros tomados en condiciones de laboratorio y en menor medida bajo las condiciones relevantes de la misión a la que van dirigidas.

El principal propósito de la construcción de la Cámara de Simulación de RLSExoMars es que se utilice como herramienta para construir nuevas bases de datos útiles al equipo científico del instrumento RLS-ExoMars.

\section{Descripción de los experimentos}

El primero de los experimentos que se presentan en este capítulo adquiere espectros de un reducido grupo de muestras representativas a diferentes temperaturas y bajo condiciones de presión tanto de RLS-ExoMars como de laboratorio terrestre para comparar.

El segundo grupo de experimentos que se presentan estudian la interacción radiación-materia desde un punto de vista térmico. Estos experimentos vienen motivados por el cambio en la forma de presentar las muestras a los instrumentos analíticos: de un cilindro sólido de muestra al polvo producido al machacar aquél. Al cambiar la forma de presentación de las muestras, se realizaron tests de irradiancia en los que se comprobó que los límites fijados $\left(0.6-1.2 \mathrm{~kW} / \mathrm{cm}^{2}\right)$ eran seguros para la distribución de grano inicial de 150-350 $\mu \mathrm{m}$. Dado que la crushing station de ExoMars produce distribuciones de grano con el límite bajo de tamaños alcanzando los $50 \mu \mathrm{m}$ o inferiores, nuevos tests eran necesarios para comprobar la validez de estos valores de irradiancia o establecer los límites necesarios para evitar la degradación de muestras termolábiles.

Para realizar esto se plantean dos formas de abordar el problema: 
1. Hacer un barrido en potencia del láser que, manteniendo el tamaño del spot láser sobre la muestra, hace variar la irradiancia sobre la misma.

2. Hacer un barrido en tamaño del spot láser que, manteniendo la potencia de emisión de éste, de forma que la irradiancia en foco se mantenga en el valor mínimo definido de $0.6 \mathrm{~kW} / \mathrm{cm}^{2}$. Para simular este efecto se desenfoca el spot sobre la muestra variando la distancia de trabajo del cabezal óptico.

El objetivo de estos experimentos no es presentar espectros de alta calidad corregidos en intensidad y línea de base. Se pretende mostrar el efecto térmico sobre la muestra en forma de imágenes y evolución del fondo del espectro y la relación señal-ruido de los picos Raman.

\section{Instrumentación}

Para todos los experimentos presentados se utilizaron:

- La Cámara de Simulación de RLS-ExoMars presentada en el Capítulo 3.

- El Simulador de Ciencia de RLS-ExoMars descrito en el Capítulo 4.

- El láser BWTEK BWN 532nm de 100mW.

- El espectrómetro BWTEK BTC-162.

- El objetivo de microscopio Nikon CFI L Plan SLWD 50X AN0.4 WD22mm.

\section{Experimento 1: Barrido en condiciones ambientales}

Para el desarrollo de este experimento se toman muestras en polvo con distribución de grano entre $50 \mu \mathrm{m}$ y $250 \mu \mathrm{m}$, la fracción baja del crusher de ExoMars, de Alunita, Yeso, Jarosita y Cuarzo.

La selección de estos minerales atiende a sus procesos de formación y lugares de ocurrencia, en algunos casos habiendo sido identificados en Marte. 
Se depositan sobre el contenedor de muestras dentro de la Cámara de Simulación de RLS-ExoMars, que se cierra y se lleva a condiciones de $\mathrm{T}=-15^{\circ} \mathrm{C}$ (en algunos casos hasta $-23^{\circ} \mathrm{C}$ ) y $\mathrm{P} \approx 7$ mbar con atmósfera de $\mathrm{CO}_{2}$. Tras ello se toman espectros Raman en foco de un grano mineral a esas condiciones y barriendo en temperatura hasta $\operatorname{los} 5^{\circ} \mathrm{C}$ con pasos de $5^{\circ} \mathrm{C}$. Estos espectros se comparan con espectros tomados en condiciones de laboratorio de las mismas muestras presentadas en la misma distribución de grano extraídos de la base de datos de espectros Raman que está construyendo la Unidad Asociada UVa-CSIC-CAB.

Se presentan los espectros calibrados de cada mineral y agrupados en la misma figura para que las posibles diferencias se hagan evidentes visualmente. Además se presenta una tabla para cada uno de estos materiales con los valores de desplazamiento Raman y de anchura a media altura de sus picos Raman más relevantes, tras haber hecho un ajuste Gaussiano - Lorenciano de los mismos mediante OPUS.

El único de los materiales analizados para el que se encuentran diferencias evidentes no sólo en estos parámetros sino también visualmente, es la Jarosita. Este hecho justifica la necesidad de creación de una base de datos de espectros Raman de minerales analizados en condiciones de RLS-ExoMars, al menos para materiales de la sensibilidad a estos cambios como la Jarosita.

\section{Experimento 2: Barrido en potencia}

Para la realización de este experimento, se preparan cada una de las muestras de la siguiente manera: (1) se coloca en el contenedor de la Cámara de Simulación de RLS-ExoMars. (2) Se cierra la cámara con ésta dentro y se establecen condiciones de $\mathrm{T}=-15^{\circ} \mathrm{C}$ y pco2 $\approx 7.5 \mathrm{mbar}$. (3) Tras ello se aplana la muestra. (4) Se aprovecha para medir la suavidad del aplanado tomando altura con respecto a la superficie del contenedor de 20 puntos espaciados $50 \mu \mathrm{m}$ en cada una de 3 líneas a lo largo de la superficie del contenedor, para verificar el cumplimiento del requisito PPL-ENG-LAB-0090 de la misión (50\% de los granos del contenedor de muestras en las $100 \mu \mathrm{m}$ primeras desde la superficie del mismo, $80 \%$ en las primeras $200 \mu \mathrm{m}$ ). (5) Se seleccionan 5 puntos a lo largo de una línea longitudinal del contenedor que contengan granos minerales sobre los que se enfoca, se toma 
una primera imagen y se realiza el siguiente proceso para cada valor de potencia desde el 5\% de la potencia máxima de salida del láser hasta el daño del mineral en pasos de 5\%: se adquiere espectro de hasta 5 minutos (modo de operación de RLSExoMars) y se toma imagen después de la adquisición. (6) Se vuelve a condiciones ambientales de laboratorio. (7) Se repite (5).

Las muestras seleccionadas para este experimento (y el siguiente) son Jarosita y Hematite. Éste también ha sido detectado en Marte desde órbita e in-situ.

La distribución de grano que se emplea para estos minerales es la fracción

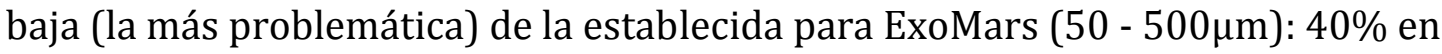
masa de granos minerales en el intervalo de tamaños de grano 50 - $125 \mu \mathrm{m}, 60 \%$ en masa en 125 - $250 \mu \mathrm{m}$.

Tanto para la Jarosita como para el Hematite se presentan las curvas de ingeniería correspondientes a la evolución de la presión y la temperatura mientras se alcanzan las condiciones de experimentación. Además se muestran imágenes del antes (antes de cerrar la cámara) y después (tras volverla a abrir) del aplanado de muestra, así como las curvas de perfil de las tres líneas de 20 puntos.

Después, para cada uno de los puntos seleccionados se presentan tablas con las imágenes tomadas tras cada exposición a una cierta irradiancia y una gráfica con los espectros Raman obtenidos a todos esos valores. Estos espectros sólo se presentan calibrados en longitud de onda y divididos por su tiempo de exposición de forma que se aprecie claramente el efecto que produce el calentamiento sobre los espectros de las muestras.

Tras presentar estas tablas para cada punto se muestra una tabla resumen en la que se listan los valores de SNR de los picos principales de cada material para evidenciar la degradación de la misma superado cierto umbral de irradiancia. Estos valores de irradiancia máxima dependen en gran medida del tamaño del grano seleccionado y el grado de cristalización, lo que ayuda a disipar más o menos eficientemente el calor recibido del láser.

Por lo general, los valores de irradiancia máxima que un grano mineral de cualquiera de los materiales es capaz de soportar, es mayor para condiciones de 
laboratorio que para condiciones de RLS-ExoMars. Esto aplica también a la calidad de los espectros obtenidos.

Como conclusión de este experimento, el valor de irradiancia promedio para el que se obtiene el mejor espectro es $0.127 \mathrm{~kW} / \mathrm{cm}^{2}$, un $20 \%$ del mínimo definido para RLS-ExoMars cuando se trabajaba en bulk, y correspondiente a un valor de potencia de salida del láser de $2.84 \mathrm{~mW}$ para un spot de $50 \mu \mathrm{m}$. Esta irradiancia puede, sin embargo, no ser suficiente con el tiempo disponible para la toma del espectro de cada punto de la muestra real para obtener un espectro útil en emisores Raman pobres.

\section{Experimento 3: Barrido en tamaño de spot}

Este experimento pretende hallar el mínimo tamaño de spot al que, manteniendo una potencia de láser constante (aquélla que en foco produce la irradiancia mínima definida para RLS-ExoMars: $0.6 \mathrm{~kW} / \mathrm{cm}^{2}$ ), hace que las muestras bajo análisis empiecen a presentar los efectos térmicos derivados de la exposición al láser.

Esta modificación del tamaño de spot se consigue desplazando del foco el sistema óptico de enfoque. De esta forma se abre el spot reduciendo la irradiancia sobre la muestra. Lamentablemente, y dado que el sistema utilizado para RLS, desenfocar el camino de excitación desenfoca en la misma medida el de colección, por lo que también se recoge menos luz Raman. De todas formas es una buena aproximación para lo que queremos demostrar.

El procedimiento de este experimento es idéntico al anterior excepto en la condición de barrido. En este caso nos movemos desde $500 \mu \mathrm{m}$ por encima del foco hasta el propio foco en pasos de $-50 \mu \mathrm{m}$, tanto para condiciones de RLS-ExoMars como para condiciones de laboratorio terrestre. Se analizan las dos mismas muestras para comparar.

Dado que este experimento se ha hecho en tiempo con el anterior, las curvas de presión y temperatura así como los perfiles de muestra coinciden. 
Nuevamente se presentan, para cada punto en cada condición ambiental, tablas con imágenes de cada paso de análisis tras la obtención del correspondiente espectro Raman y una gráfica con los espectros combinados. Las imágenes de cada paso se toman en foco para observar el daño producido.

En algunos casos durante el análisis de ciertos puntos se observa que los granos minerales desaparecen del campo de observación. Esto se achaca a la emisión y expansión de los gases producidos por calentamiento de los granos minerales, o a una reducción de volumen por la transformación química del grano mineral en observación.

A modo de recolección de datos, se vuelven a presentar tablas con información de la SNR de los picos principales para ambos minerales para cada punto analizado y a cada altura desde foco. Se muestra también la equivalencia de esta altura con el tamaño de spot y la irradiancia sobre el grano.

La conclusión de este experimento es que haría falta una irradiancia de $0.1 \mathrm{~kW} / \mathrm{cm}^{2}$ (en consistencia con la hallada con el experimento anterior), que correspondería a un spot de $\sim 130 \mu$ m de diámetro si trabajamos a la misma potencia que lo haría un spot de $50 \mu \mathrm{m}$ a la mínima irradiancia de RLS-ExoMars $\left(0.6 \mathrm{~kW} / \mathrm{cm}^{2}\right)$.

La termolabilidad y los efectos térmicos son asuntos delicados. Dependen de factores, muchos de los cuales están fuera de nuestro control, como lo son las propiedades de la muestra: ratio superficie-a-volumen, grado de cristalización del grano mineral, coeficiente de absorción del material, etc. Las únicas limitaciones que podemos imponer a nuestro sistema son instrumentales y metodológicas. No sólo la potencia del láser y/o el tamaño de spot tienen que ser cuidadosamente elegidos, sino que el tiempo de irradiación de una muestra termolábil contribuye a su degradación, incluso en valores de irradiancia considerados seguros. Es por ello que algoritmos de reducción de fluorescencia por irradiación de muestra, por citar alguno, han de ser testados concienzudamente e incluidos sus resultados en la valoración del diseño instrumental. 
Existen soluciones operativas para no tener que modificar los requisitos centíficos y por tanto los valores técnicos y pasan por la definición de algoritmos de operación que detecten a través del espectro daños en la muestra conducentes a espectros no óptimos o incluso dañados contemplando el descarte y paso a otro grano. Aunque a primera vista parecen alternativas viables, hay que ser muy cuidadosos en su definición pues pueden hacer que el instrumento pase por alto o dañe irremediablemente puntos de muestra únicos y valiosísimos desde el punto de vista de los objetivos de la misión, como serían los que contuvieran rastros biológicos.

\section{Capítulo 6 - Conclusiones}

\section{Conclusiones de esta tesis doctoral}

Las conclusiones de esta tesis se pueden resumir brevemente en tres puntos:

1. Se han diseñado y construido una Cámara de Simulación de RLS-ExoMars en combinación con un Simulador de Ciencia de RLS-ExoMars como herramientas de apoyo para la ciencia de soporte del instrumento RLS-ExoMars.

La Cámara de Simulación de RLS-ExoMars es capaz de enfriar la muestra a temperaturas de operación $\left(<-5^{\circ} \mathrm{C}\right)$ e inferiores. También es capaz de recrear en su interior las presiones y temperaturas atmosféricas que las muestras experimentarán dentro del rover de ExoMars, en el Analytical Laboratory Drawer (ALD). Tiene una cuchilla motorizada que permite, como operación de preparación para el análisis, aplanar la superficie de la muestra en polvo previamente depositada en el contenedor de muestra del interior de la cámara. El Simulador de Ciencia de RLS-ExoMars es capaz de posicionar el cabezal óptico Raman a lo largo y ancho de la superficie del contenedor dentro de la cámara y acceder a 100x visual y espectroscópicamente a la muestra a través de la ventana óptica instalada en la tapa superior de la cámara.

Las condiciones ambientales y el posicionamiento del cabezal Raman son establecidas y monitorizadas mediante un software de PC diseñado para tal efecto. El proceso de aplanado se comanda manualmente a través de una placa 
electrónica y un software embebido también diseñados ex profeso para este proyecto.

2. De la comparativa de análisis Raman bajo condiciones marcianas y terrestres para determinar la necesidad de crear una base de datos espectral Raman específica de la misión, se puede concluir que para muestras termolábiles como la Jarosita esta base de datos es de una importancia fundamental. Estas muestras termolábiles se comportan de forma distinta bajo condiciones de presión y temperatura diferentes debido al calentamiento térmico inducido por el láser, lo que puede provocar transformaciones. Una base de datos que contenga todos estas posibilidades para diferentes distribuciones de grano será una herramienta potente para la explotación de datos en el contexto de la misión ExoMars.

3. Con respecto a estas transformaciones térmicas, definir valores de irradiancia sobre las muestras, con énfasis en muestras termolábiles, es la tarea más importante para evitar efectos térmicos que impidan obtener espectros Raman de alta calidad de los minerales bajo estudio. Se ha concluido que, para el nuevo escenario de distribuciones de tamaño de grano en el que la cola de tamaños menores se extiende hasta las $50 \mu \mathrm{m}$ e inferior, la irradiancia tiene que ser reducida con respecto a los valores actuales. Se debe hallar un compromiso entre la potencia del láser y el comportamiento Raman global de la misión de forma que se puedan analizar todo tipo de muestras, especialmente aquéllas que justifican y demostrarán los objetivos de la misión. Hay varias formas de hacer esto: reducir la potencia del láser, agrandar el tamaño de spot, crear algoritmos de detección de daños, etc.

\section{Trabajo futuro}

Teniendo esta herramienta disponible para hacer experimentos relacionados con el instrumento RLS bajo condiciones de ExoMars, incontables son los experimentos que se pueden imaginar para contiunar haciendo ciencia, definiendo el instrumento y su operación, para preparar esta misión y muchas otras por llegar. 
La tarea más urgente a realizar en relación con la ciencia de ExoMars es la compilación de la base de datos de espectros RLS-ExoMars contra la cual comparar los espectros recibidos una vez el rover los mande desde Marte.

Nuevos tests a realizar incluyen cómo el instrumento se enfrentará a muestras fluorescentes. Esto cae dentro del campo del modo de operación del instrumento bajo las condiciones relevantes de RLS-ExoMars, un trabajo que empezó con el precursor del Simulador de Ciencia de RLS-ExoMars.

En paralelo, es la hora de comenzar con la integración de la versión final del instrumento RLS que se está desarrollando en INTA con una nueva Cámara de Simulación de RLS-ExoMars basada en la aquí presentada. Esto ayudará a los científicos de RLS a restringir los requisitos científicos del instrumento como el establecimiento de los límites de irradiancia, la determinación de los valores de relación señal-ruido que pueden obtenerse y la fijación de los parámetros de adquisición que optimizarán el tiempo y los recursos de la misión para devolver el mejor material analítico que la ciencia pueda conseguir para alcanzar conclusiones valiosas acerca de la vida en Marte.

La Cámara de Simulación de RLS-ExoMars está preparada (cambiando algunos elementos) para la simulación de otros ambientes en Marte. Bajando la temperatura de la muestra a valores cercanos a $-90^{\circ} \mathrm{C}$ y calentándola desde ahí podemos entender los procesos de transformación que sufren las muestras entre el subsuelo y el ALD de ExoMars.

Y yendo más allá, es posible simular ambientes superficiales de otros cuerpos planetarios del Sistema Solar, previendo futuras misiones de exploración planetaria.

Pero esta herramienta no sólo puede servir a la ciencia en el campo de la geoquímica o incluso de la exploración planetaria. Esta herramienta puede utilizarse para analizar minerales y organismos vivos pero también materiales naturales y sintéticos para la industria y estudiar su comportamiento bajo condiciones de baja presión y temperatura. 